\title{
Highly Site-Selective Direct C-H Bond Functionalization of Unactivated Arenes with Propargyl a-Aryl-a-diazoacetates via Scandium Catalysis
}

Balu S. Navale, Debasish Laha, Subhrashis Banerjee, Kumar Vanka, Prof. Dr. Ramakrishna G. Bhat Submitted date: 19/04/2021 - Posted date: 20/04/2021

Licence: CC BY-NC-ND 4.0

Citation information: Navale, Balu S.; Laha, Debasish; Banerjee, Subhrashis; Vanka, Kumar; Bhat, Prof. Dr. Ramakrishna G. (2021): Highly Site-Selective Direct C-H Bond Functionalization of Unactivated Arenes with Propargyl a-Aryl-a-diazoacetates via Scandium Catalysis. ChemRxiv. Preprint. https://doi.org/10.26434/chemrxiv.14447940.v1

Highly chemo- and regio-selective $\mathrm{C}-\mathrm{H}$ bond functionalization of unactivated arenes with propargyl a-aryl-a-diazoacetates is developed using scandium catalysis. Variety of unactivated, mildly deactivated and electronically activated arenes are functionalized using this protocol. We have explored the novel combination of scandium triflate and propargyl a-aryl-a-diazoacetate as catalyst-reagent system for the effective $\mathrm{C}-\mathrm{H}$ bond functionalization. The protocol avoids the use of expensive catalysts and practicality of the protocol has been demonstrated by the gram scale synthesis of very useful a-diarylacetates including antispasmodic drug-adephenine.

File list (2)

Manuscript.pdf (1.37 MiB) view on ChemRxiv • download file 


\title{
Highly Site-Selective Direct $\mathrm{C}-\mathrm{H}$ Bond Functionalization of Unactivated Arenes with Propargyl $\alpha$-Aryl- $\alpha$-diazoacetates via Scandium Catalysis
}

\author{
Balu S. Navale, ${ }^{a}$ Debasish Laha, ${ }^{a}$ Subhrashis Banerjee,,${ }^{b, c}$ Kumar Vanka, ${ }^{b, c}$ and Ramakrishna G. \\ Bhat $^{\text {a* }}$
}

Abstract: Highly chemo- and regio-selective $\mathrm{C}-\mathrm{H}$ bond functionalization of unactivated arenes with propargyl $\alpha$-aryl- $\alpha$ diazoacetates is developed using scandium catalysis. Variety of unactivated, mildly deactivated and electronically activated arenes are functionalized using this protocol. We have explored the novel combination of scandium triflate and propargyl $\alpha$-aryl- $\alpha$-diazoacetate as catalyst-reagent system for the effective $\mathrm{C}-\mathrm{H}$ bond functionalization. The protocol avoids the use of expensive catalysts and practicality of the protocol has been demonstrated by the gram scale synthesis of very useful $\alpha$-diarylacetates including antispasmodic drug-adephenine.

Catalytic $\mathrm{C}-\mathrm{H}$ bond functionalization is one of the most important and viable methods for the synthesis of complex organic molecules. ${ }^{[1]}$ Developing catalytic methods for the chemo- and regioselective $\mathrm{C}-\mathrm{H}$ bond functionalization is highly desirable but challenging task for the synthetic community. Substituted arenes are widespread and present in various biologically active compounds, and drug molecules. ${ }^{[2]}$ Owing to the importance the chemo- and regioselective $\mathrm{C}-\mathrm{H}$ bond functionalization of arenes are highly desirable to access different bioactive compounds in minimum number of steps. Aromatic $\mathrm{C}-\mathrm{H}$ bond functionalization using $\alpha$-diazocarbonyl compounds is one of the important approaches to access substituted arenes. ${ }^{[3]}$ As the $\alpha$-diazocarbonyl compounds can be easily prepared from readily available starting materials, the use of $\alpha$-diazocarbonyl compounds in aromatic $\mathrm{C}-\mathrm{H}$ bond functionalization made remarkable progress over the years. ${ }^{[3,4,9]}$ Efforts have been made to make this method more practical with modifying catalysts, $\alpha$-diazocarbonyl reagents for the aromatic $\mathrm{C}-\mathrm{H}$ bond functionalization. The transition metal complexes of rhodium,$^{[4]}$ gold,${ }^{[5]}$ copper, ${ }^{[6]}$ and iron ${ }^{[7]}$ have been widely used for the aromatic $\mathrm{C}-\mathrm{H}$ bond functionalization reactions with $\alpha$ diazocarbonyl compounds. Recently Ṕerez and co-workers utilized manganese catalyst for the aromatic $\mathrm{C}-\mathrm{H}$ bond functionalization using $\alpha$-diazocarbonyl compounds. ${ }^{[8]}$ Likewise, transition metal-free $\mathrm{C}-\mathrm{H}$ bond functionalization reactions of electron rich arenes have been also explored..$^{[9]}$ Recently, Yu et al. reported a highly chemoselective $\mathrm{C}-\mathrm{H}$ bond functionalization of phenols with $\alpha$-aryl- $\alpha$-diazoacetates by exploring the gold catalysis. ${ }^{[5 \mathrm{~d}]}$ Interestingly, under the reaction conditions, $\mathrm{C}-\mathrm{H}$ insertion occurred exclusively, and the $\mathrm{O}-\mathrm{H}$ insertion product was not obtained. This was the first protocol wherein; the unprotected phenols were effectively functionalized by using $\alpha$ aryl- $\alpha$-diazoacetates and gold catalyst and this transformation proved to be highly para selective. In 2014, Shi and co-workers reported the ligand-controlled gold-catalyzed $\mathrm{C}-\mathrm{H}$ bond functionalization of electronically activated arenes with $\alpha$-aryl- $\alpha$ diazoacetates (Fig 1 A). ${ }^{[5 e]}$ Various electronically rich arenes

[a]Department of Chemistry, Main Building, Indian Institute of Science Education and Research (IISER)-Pune, Dr. Homi Bhabha Road, Pashan, Pune, 411008, Maharashtra, India Email: rgb@iiserpune.ac.in [b]Physical and Materials Chemistry Division, Pune, 411008, India [c]Academy of Scientific and Innovative Research (AcSIR), Ghaziabad201002, India

Supporting information for this article is given via a link at the end of the document. ((Please delete this text if not appropriate)) underwent the $\mathrm{C}-\mathrm{H}$ bond functionalization reaction with the $\alpha$ aryl- $\alpha$-diazoacetates in the presence of gold catalyst to afford corresponding desired $\alpha$-diarylacetates. However, this method had a limited substrate scope as the protocol worked only with the electronically activated arenes. In 2017, Zhang and coworkers reported the para selective $\mathrm{C}-\mathrm{H}$ alkylation of arenes with 2,2,2-trifluoroethyl $\alpha$-aryl- $\alpha$-diazoesters via gold catalysis (Fig 1B). ${ }^{[5]]}$ However, the $\mathrm{C}-\mathrm{H}$ alkylation of benzene and unactivated arenes worked only with 2,2,2-trifluoroethyl $\alpha$-(4-trifluormethyl)phenyl- $\alpha$-diazoactate. The presence of electron withdrawing group such as trifluoromethyl on the aryl group of diazoester ( $p$ position) was found to be essential for this transformation. Very recently, Shi and co-workers developed an another method for the $\mathrm{C}-\mathrm{H}$ bond functionalization of arenes with $\alpha$-aryl- $\alpha$ diazoacetates in the presence of gold catalysttriphenylphosphine gold(I) trifluoromethanesulfonate (Fig $1 \mathrm{C}){ }^{[59]}$ In this protocol they used $\alpha$-aryl- $\alpha$-diazoacetates containing electron deactivating substituents such as halogens on the phenyl ring. These substituents proved to be important for the chemoselectivity and site-selectivity of the transformation. The desired products $\alpha$-diarylacetates were obtained in good to excellent yields. Although gold catalysts have been well explored for the synthesis of $\alpha$-diarylacetates, however, usually they are expensive, and not all of these gold catalysts are commercially available..$^{[5]}$ The synthesis of $\alpha$-diarylacetates using unactivated arenes and electronically deactivated arenes by modifying the catalyst and modulating the diazocarbonyl reagent system electronically remains an interesting and challenging task for the synthetic chemists. ${ }^{[5 f, 5 g]}$ Also, it would be highly interesting and challenging to explore the $\mathrm{C}-\mathrm{H}$ bond functionalization of unactivated arenes with $\alpha$-phenyl- $\alpha$ diazoacetates or aryl diazoesters containing unsubstituted phenyl group. It would also be interesting to tap the potential of the unexplored early transition metal-based catalysts for the site selective $\mathrm{C}-\mathrm{H}$ bond functionalization of unactivated arenes.

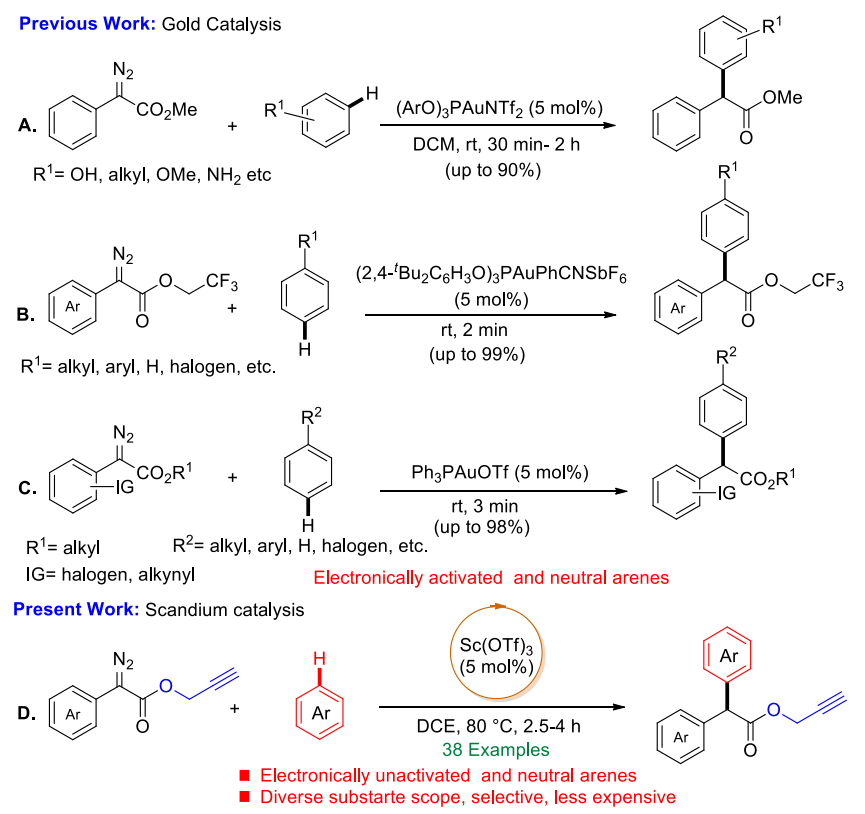

Fig. 1. $\mathrm{C}-\mathrm{H}$ functionalization of arenes with diazoacetates 
Some of the late transition metals have been explored for the $\mathrm{C}$ $\mathrm{H}$ bond functionalization for their efficiency. Interestingly, to the best of our knowledge, early transition elements have been seldom explored in the literature for the $\mathrm{C}-\mathrm{H}$ bond functionalization. However, the synergistic combination of reagent and catalyst is very crucial for the effective $\mathrm{C}-\mathrm{H}$ bond functionalization. As a part of our ongoing efforts to explore the diazocarbonyl compounds for the effective $\mathrm{C}-\mathrm{H}$ bond functionalization using early transition elements, we planned to explore the suitable combination of the catalyst and the modified diazocarbonyl reagent. We believed that the reagents with suitable electronic properties may be necessary for the effective $\mathrm{C}-\mathrm{H}$ bond functionalization using early transition metal catalysts. Herein, we report the novel scandium catalyzed $\mathrm{C}-\mathrm{H}$ bond functionalization of unactivated arenes and mildly deactivated arenes with propargyl $\alpha$-aryl- $\alpha$-diazoacetates as new robust reagents to synthesize useful and biologically important $\alpha$ diarylacetate compounds in good to excellent yields (Fig $1 \mathrm{D}$ ).

Table 1. Optimization of the reaction conditions ${ }^{[a-d]}$

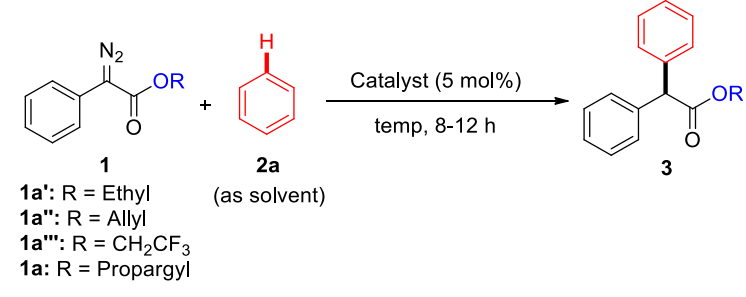

\begin{tabular}{|c|c|c|c|c|c|}
\hline Entry & Catalyst & $\mathbf{R}$ & $\begin{array}{c}\text { Time } \\
{[\mathrm{h}]}\end{array}$ & $\begin{array}{c}\text { Temp } \\
{\left[{ }^{\circ} \mathrm{C}\right]}\end{array}$ & $\begin{array}{c}\text { Yield }{ }^{[b, c, d]} \\
{[\%]}\end{array}$ \\
\hline 1 & $\mathrm{Rh}_{2}(\mathrm{OAc})_{4}$ & Ethyl & 12 & $\mathrm{rt}$ & $\mathrm{DC}$ \\
\hline 2 & $\mathrm{Rh}_{2}(\mathrm{OAc})_{4}$ & Ethyl & 4 & 80 & $\mathrm{DC}$ \\
\hline 3 & $\mathrm{Cu}(\mathrm{OTf})_{2}$ & Ethyl & 12 & $\mathrm{rt}$ & $\mathrm{DC}$ \\
\hline 4 & $\mathrm{Cu}(\mathrm{OTf})_{2}$ & Ethyl & 4 & 80 & trace \\
\hline 5 & $(\mathrm{ArO})_{3} \mathrm{PAuNTf}_{2}$ & Ethyl & 12 & $\mathrm{rt}$ & DC \\
\hline 6 & $\mathrm{Sc}(\mathrm{OTf})_{3}$ & Ethyl & 12 & $\mathrm{rt}$ & $N R^{d}$ \\
\hline 7 & $\mathrm{Sc}(\mathrm{OTf})_{3}$ & Ethyl & 12 & 80 & $21 \%$ \\
\hline 8 & LAuPhCNSbF 6 & Ethyl & 12 & $\mathrm{rt}$ & NR \\
\hline 9 & LAuPhCNSbF 6 & Ethyl & 12 & 80 & trace \\
\hline 10 & $\mathrm{Sc}(\mathrm{OTf})_{3}$ & Allyl & 12 & 80 & $33 \%$ \\
\hline 11 & $\mathrm{Sc}(\mathrm{OTf})_{3}$ & $\mathrm{CH}_{2} \mathrm{CF}_{3}$ & 8 & 80 & $52 \%$ \\
\hline $12^{*}$ & LAuPhCNSbF6 & $\mathrm{CH}_{2} \mathrm{CF}_{3}$ & $20 \mathrm{~min}$ & rt & $8 \%$ \\
\hline 13 & $\mathrm{Sc}(\mathrm{OTf})_{3}$ & Propargyl & 12 & $\mathrm{rt}$ & NR \\
\hline 14 & $\mathrm{Sc}(\mathrm{OTf})_{3}$ & Propargyl & 8 & 80 & $70 \%$ \\
\hline 15 & $\mathrm{Cu}(\mathrm{OTf})_{2}$ & Propargyl & 12 & 80 & trace \\
\hline 16 & $(\mathrm{ArO})_{3} \mathrm{PAuNTf}_{2}$ & Propargyl & 12 & rt & DC \\
\hline 17 & $(\mathrm{ArO})_{3} \mathrm{PAuNTf}_{2}$ & Propargyl & 4 & 80 & DC \\
\hline 18 & $\mathrm{Rh}_{2}(\mathrm{OAc})_{4}$ & Propargyl & 12 & $\mathrm{rt}$ & DC \\
\hline 19 & $\begin{array}{l}\text { Dichloro( } p \text {-cymene) } \\
\text { ruthenium(II) dimer }\end{array}$ & Propargyl & 12 & rt & $\mathrm{DC}$ \\
\hline 20 & $\ln (\mathrm{OTf})_{3}$ & Propargyl & 12 & rt & NR \\
\hline 21 & $\ln (\mathrm{OTf})_{3}$ & Propargyl & 12 & 80 & $47 \%$ \\
\hline 22 & $\mathrm{Bi}(\mathrm{OTf})_{3}$ & Propargyl & 12 & rt & NR \\
\hline 23 & $\mathrm{Bi}(\mathrm{OTf})_{3}$ & Propargyl & 12 & rt & $10 \%$ \\
\hline 24 & $Y(O T f)_{3}$ & Propargyl & 12 & 80 & $20 \%$ \\
\hline 25 & $\mathrm{Yb}(\mathrm{OTf})_{3}$ & Propargyl & 12 & 80 & trace \\
\hline 26 & $\mathrm{FeCl}_{3} .6 \mathrm{H}_{2} \mathrm{O}$ & Propargyl & 12 & rt & NR \\
\hline 27 & $\mathrm{FeCl}_{3} .6 \mathrm{H}_{2} \mathrm{O}$ & Propargyl & 12 & 80 & $19 \%$ \\
\hline 28 & Triflic acid & Propargyl & 12 & $\mathrm{rt}$ & $21 \%$ \\
\hline 29 & Triflic acid & Propargyl & 12 & 80 & $23 \%$ \\
\hline 30 & No Catalyst & Propargyl & 12 & 80 & NR \\
\hline
\end{tabular}

[a] Reaction conditions: a solution of $\alpha$-aryl- $\alpha$-diazoacetate $1(0.5 \mathrm{mmol}, 1$ equiv.) in $1 \mathrm{~mL}$ benzene was added to a solution of catalyst ( $5 \mathrm{~mol} \%)$ and benzene $2 \mathrm{a}(1.5 \mathrm{~mL})$ under inert atmosphere; the reaction was monitored by TLC. [b] Isolated yield after purification by column chromatography. [c] DC- decomposed, [d] NR- no reaction, $L=\left(2,4-{ }^{-} \mathrm{Bu}_{2} \mathrm{C}_{6} \mathrm{H}_{3} \mathrm{O}\right){ }_{3} \mathrm{P}$. * $1 \mathrm{a}$ ", was completely consumed in $20 \mathrm{~min}$.
In order to explore the feasibility of the proposed strategy, we commenced our study using ethyl $\alpha$-phenyl- $\alpha$-diazoacetate 1a' and benzene $\mathbf{2} \mathbf{a}$ as the model substrates in presence of various metal catalysts at room temperature to refluxing conditions $\left(80{ }^{\circ} \mathrm{C}\right)$ in dry benzene (entries $1-7$, Table 1$)$. Rhodium, copper and gold catalysts found to be ineffective for the $\mathrm{C}-\mathrm{H}$ bond functionalization of benzene at room temperature as well as at refluxing conditions (entries $1-5$, Table 1 ). Later, when we used catalytic amount $(5 \mathrm{~mol} \%)$ of $\mathrm{Sc}(\mathrm{OTf})_{3}$ for this transformation at room temperature, the starting material ethyl $\alpha$-phenyl- $\alpha$ diazoacetate 1a' remained intact even after prolonged reaction time $(12 \mathrm{~h}$, entry 6 , Table 1$)$. Interestingly, the reaction of ethyl $\alpha$-phenyl- $\alpha$-diazoacetate $1 \mathrm{a}^{\prime}$ and benzene $2 \mathrm{a}$ in the presence of catalytic amount of $\mathrm{Sc}(\mathrm{OTf})_{3}(5 \mathrm{~mol} \%)$ at elevated temperature $\left(80^{\circ} \mathrm{C}\right)$, afforded the desired $\mathrm{C}-\mathrm{H}$ bond functionalization product ethyl 2,2-diphenylacetate $3 a^{\prime}$ a in $31 \%$ yield (entry 7 , Table 1 ). Later, reaction of ethyl $\alpha$-phenyl- $\alpha$-diazoacetate 1a' and benzene $2 a$ in presence of gold catalyst under rt to elevated temperature did afford the desired product (Table 1, entries 8,9). Encouraged by this preliminary result (entry 7 ), we synthesized allyl $\alpha$-phenyl$\alpha$-diazoacetate $1 \mathrm{a} "$. The treatment of allyl $\alpha$-phenyl- $\alpha$ diazoacetate $1 \mathbf{a}^{\prime}$ with benzene $\mathbf{2 a}$ in presence of catalytic amount of $\mathrm{Sc}(\mathrm{OTf})_{3}(5 \mathrm{~mol} \%)$ in dry benzene at $80{ }^{\circ} \mathrm{C}$ afforded the desired product allyl 2,2-diphenylacetate 3a"a in slightly improved yield in comparison to the ethyl $\alpha$-phenyl- $\alpha$ diazoacetate 1a' (33\%, Table 1, entry 10). Further, we planned to explore the reactivity of 2,2,2-trifluoroethyl $\alpha$-phenyl- $\alpha$ diazoacetate 1a"' under scandium catalysis. Gratifyingly, the reaction of $1 \mathbf{a}^{\prime \prime}$ and $2 \mathbf{a}$ in presence of $\mathrm{Sc}(\mathrm{OTf})_{3}(5 \mathrm{~mol} \%)$ at 80 ${ }^{\circ} \mathrm{C}$ afforded the desired product $3 \mathrm{a}$ "'a in $52 \%$ yield (Table 1 , entry 11). However, surprisingly, reaction of 2,2,2-trifluoroethyl $\alpha$-phenyl- $\alpha$-diazoacetate $1 \mathrm{a}^{\prime \prime}$ and benzene under the reported gold catalysis furnished the desired product in poor yield (Table 1 , entry 12).

The Initial results of the scandium catalyzed $\mathrm{C}-\mathrm{H}$ bond functionalization proved to be encouraging. However, based on our initial observations, we hypothesized that ethyl/allyl/2,2,2trifluoroethyl $\alpha$-phenyl- $\alpha$-diazoacetate reagents 1 (1a',1a" and $1 a^{\prime \prime \prime) ~ m a y ~ b e ~ r e l a t i v e l y ~ l e s s ~ r e a c t i v e ~(d o n o r / a c c e p t o r ~ t y p e ~} \alpha$ diazo acetates) to afford the desired products $\alpha$-diarylacetates 3 in higher yields. Also, the catalytic activity of scandium triflate and low reactivity of unactivated arene such as benzene $\mathbf{2 a}$ towards $\mathrm{C}-\mathrm{H}$ bond functionalization may be the other prime cause of lower yields of 3a'a and 3a"a. It is known in the literature that propargyl moiety is a weakly electron-withdrawing group ${ }^{13 a}$ and in this regard, we planned to explore the effect of propargyl group in $\mathrm{C}-\mathrm{H}$ bond functionalization of unactivated arenes. To explore the feasibility and to enhance the reactivity of $\alpha$-aryl- $\alpha$-diazoacetate towards the $\mathrm{C}-\mathrm{H}$ bond functionalization, we synthesized the propargyl $\alpha$-phenyl- $\alpha$-diazoacetate $\mathbf{1 a}$ containing electron withdrawing ${ }^{13 a}$ propargyl group as an ester part that can be removed after the functionalization.

To validate our hypothesis, we planned to explore the reactivity of propargyl $\alpha$-phenyl- $\alpha$-diazoacetate $1 \mathbf{1 a}$. The reaction of $1 \mathrm{a}$ with benzene $2 a$ in presence of presence of $\mathrm{Sc}(\mathrm{OTf})_{3}$ at room temperature did afford the desired product (Table 1, entry 13). Gratifyingly, the reaction of propargyl $\alpha$-phenyl- $\alpha$-diazoacetate $\mathbf{1 a}$ and benzene $\mathbf{2 a}$ afforded the desired $\mathrm{C}-\mathrm{H}$ bond functionalization product propargyl 2,2-diphenylacetate 3aa in $70 \%$ yield in the presence of $\mathrm{Sc}(\mathrm{OTf})_{3}(5 \mathrm{~mol} \%)$ at $80{ }^{\circ} \mathrm{C}$ in $8 \mathrm{~h}$ (entry 13, Table 1). Further we planned to explore the reactivity of 1a in presence of different metal catalysts at room temperature to elevated temperature in dry benzene 2a (Table 1 ,entries 15-27). It is interesting to note copper as well as gold catalysts were found to be unsuccessful in affording the desired product (Table 1, entries 15-17). Rhodium as well ruthenium catalysts also found to be ineffective for the desired direct $\mathrm{C}-\mathrm{H}$ bond functionalization of benzene 2a (Table 1, entries 18-19). The reaction of benzene $2 a$ with propargyl $\alpha$-phenyl- $\alpha$ diazoacetate $1 \mathrm{a}$ in the presence of other metal catalysts afforded the direct $\mathrm{C}-\mathrm{H}$ bond functionalization product $3 \mathrm{aa}$ either in low or moderate yields, in some cases starting materials 
remained intact (Table 1, entries 20-27). We observed that the most of the catalysts were found to be ineffective to catalyse the desired transformation. In few cases the reactions led to the decomposition of the reagent. Also, varying the reaction temperatures from rt to $80{ }^{\circ} \mathrm{C}$ did not influence the outcome of the reaction in most of the cases. In order to confirm the role $\mathrm{Sc}(\mathrm{OTf})_{3}$ as a catalyst and to rule out the possible formation of trace amount of triflic acid in situ by the hydrolysis of $\mathrm{Sc}(\mathrm{OTf})_{3}$ if any due to the moisture and its subsequent catalysis, we carried out the reaction of $\mathbf{1 a}$ and $\mathbf{2} \mathbf{a}$ in presence of catalytic amount triflic acid (Table 1, entries 28-29). However, the desired product 3aa was formed in poor yields. The $\mathrm{C}-\mathrm{H}$ bond functionalization did not work in the absence of catalyst $\mathrm{Sc}(\mathrm{OTf})_{3}$ (Table 1, entry 30).

Scheme 1. Scope of Diazoacetates and Arenes

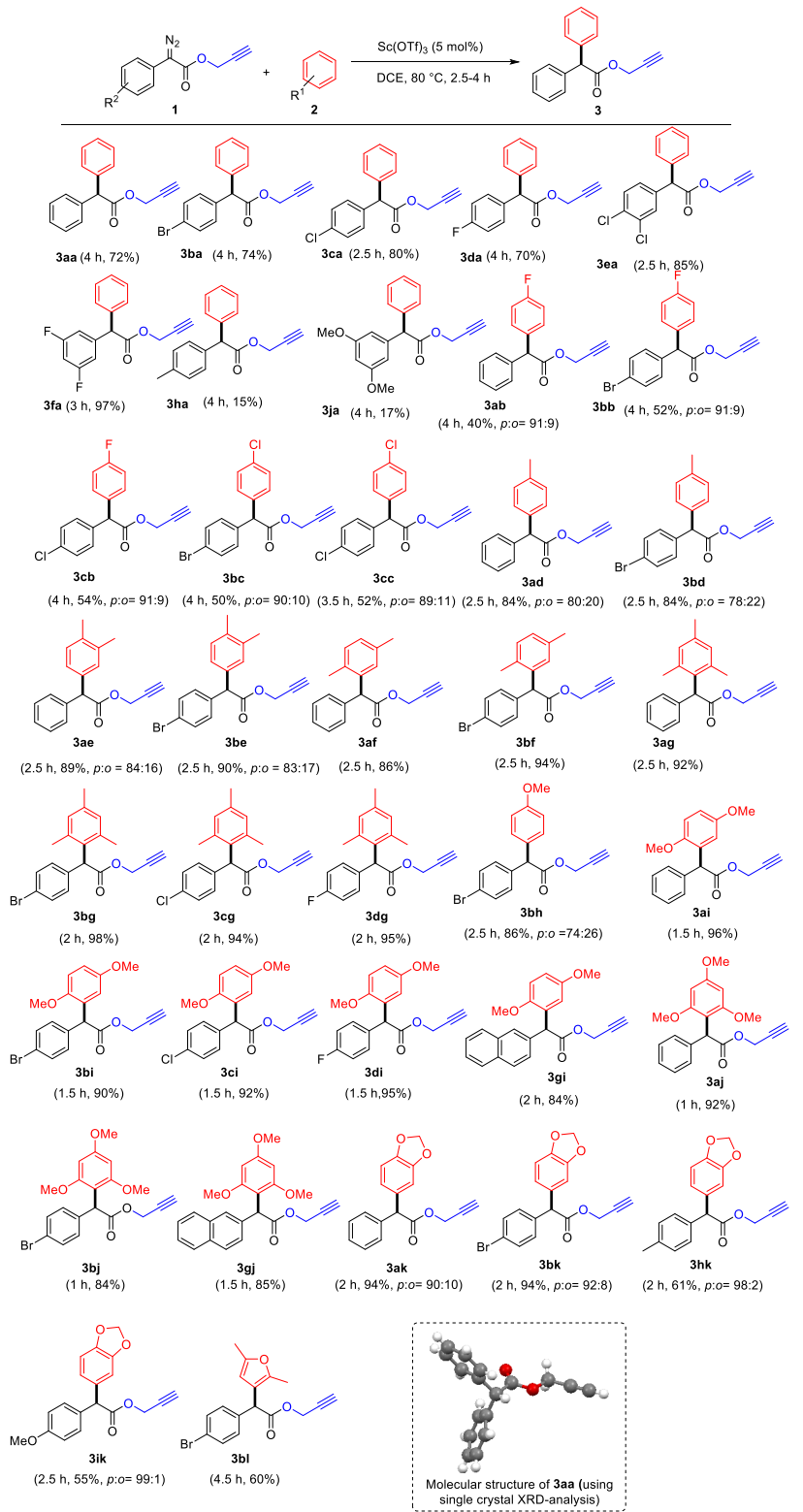

${ }^{[a]}$ Reaction conditions: propargyl $\alpha$-aryl- $\alpha$-diazoacetates 1 ( $0.5 \mathrm{mmol}$, 1equiv.), arene 2 (10 equiv.) and $\mathrm{Sc}(\mathrm{OTf})_{3}(5 \mathrm{~mol} \%)$ was added in $\mathrm{DCE}(2.5 \mathrm{~mL})$ under inert atmosphere and reaction mixture was refluxed at $80{ }^{\circ} \mathrm{C}$; the reaction was monitored by TLC. ${ }^{[\mathrm{b}]}$ /solated yield after purification by column chromatography (Time and yields are given in parenthesis).

Encouraged by the initial success, we screened the reaction in different solvents at varying temperature and catalytic loading of $\mathrm{Sc}(\mathrm{OTf})_{3}$ to optimize the synthesis of desired product 3aa in highest yield (Appendix-I, ESI). Dichloroethane proved to be the optimum solvent by affording the desired product 3aa in $72 \%$ yield at $80{ }^{\circ} \mathrm{C}$ in $4 \mathrm{~h}$ (Appendix-I, Entry 4, ESI). However, the polar solvents such as DMF and acetonitrile found to be disadvantageous (entries 5-6, Appendix-I). Solvents such as ethyl acetate, nitromethane, and dichloromethane afforded the desired product 3aa in low to moderate yields (Appendix I, entries 1-3, ESI). We also observed that the lower catalyst loading of $\mathrm{Sc}(\mathrm{OTf})_{3}(2.5 \mathrm{~mol} \%)$ afforded the desired product 3aa in moderate yield in $8 \mathrm{~h}$ (Appendix-I, entry $7, \mathrm{ESI}$ ).

We further looked into the possible effect of co-catalyst in this transformation. We screened two different silver co-catalysts along with the $\mathrm{Sc}(\mathrm{OTf})_{3}$ to optimize the reaction (Appendix-II, ESI). Interestingly, both $\mathrm{AgSbF}_{6}$ and $\mathrm{AgOTf}$ found to be ineffective and led to the formation of the desired product 3aa in lower yields (Appendix-II, entry 1-5, ESI). However, the reaction worked smoothly in the absence of co-catalyst to afford the desired product 3aa in good yield (72\% yield, Appendix-II entry 6 , $\mathrm{ESI}$ ). This very important to note that $\mathrm{C}-\mathrm{H}$ bond functionalization of benzene and unactivated arenes is quite challenging and many efforts have been made earlier by different strategies. Further, the molecular structure of the compound 3aa was unambiguously confirmed by single crystal X-ray analysis. ${ }^{[14]}$

Based on comprehensive screening propargyl $\alpha$-phenyl- $\alpha$ diazoacetate 1a. benzene 2a (10 equiv.), Sc(OTf) $3(2.5 \mathrm{~mol} \%)$ at $80^{\circ} \mathrm{C}$ under inert atmosphere proved to be the optimum reaction condition.

Having optimized the reaction conditions, we then focussed our attention to explore the reagent scope of this $\mathrm{C}-\mathrm{H}$ bond functionalization protocol using unactivated arene such as benzene 2a with different the propargyl $\alpha$-aryl- $\alpha$-diazoacetate reagents 1 . Benzene $2 \mathrm{a}$ reacted with propargyl $\alpha$-aryl- $\alpha$ diazoacetates (1a-1f, 1h, 1j) smoothly to afford the desired products (3aa-3fa, 3ha, 3ja) in excellent yields (up to $97 \%$ yield, Scheme 1). Propargyl $\alpha$-aryl- $\alpha$-diazoacetates (1b-1f) with weak electron-withdrawing substituents on the phenyl ring proved to be very good reagents for the $\mathrm{C}-\mathrm{H}$ bond functionalization of benzene $\mathbf{2 a}$ to afford the desired products (3ba-3fa) in good to excellent yields (up to $97 \%$ yield, Scheme 1 ). While the $\alpha$-aryl- $\alpha$ diazoacetates $(\mathbf{1 h}, \mathbf{1 j})$ with electron-donating substituents on the phenyl ring greatly reduced the reactivity of propargyl $\alpha$-aryl- $\alpha$ diazoacetates towards the $\mathrm{C}-\mathrm{H}$ bond functionalization under the optimum reaction conditions to afford the desired products ( $3 \mathrm{ha}$, 3ja) in lower yields (Scheme 1). These observations indicated that electron-withdrawing substituents on the phenyl ring of propargyl $\alpha$-aryl- $\alpha$-diazoacetates play a major role in tuning the reactivity of the reagent. Propargyl $\alpha$-aryl- $\alpha$-diazoacetates found to be most suitable reagents for the effective $\mathrm{C}-\mathrm{H}$ bond functionalization of an unactivated arene $\mathbf{2 a}$ in presence $\mathrm{Sc}(\mathrm{OTf})_{3}$.

After the initial success on the $\mathrm{C}-\mathrm{H}$ bond functionalization of an unactivated arene such as benzene, we turned our attention to explore the relatively more challenging mildly deactivated arenes. Gratifyingly, the reactions of fluorobenzene $\mathbf{2 b}$ with different propargyl $\alpha$-aryl- $\alpha$-diazoacetates $(\mathbf{1 a}, \mathbf{1 b}, \mathbf{1 c})$ under the optimal reaction conditions afforded the corresponding desired products (3ab, $\mathbf{3 b b}, \mathbf{3} \mathbf{c b}$ ) in moderate yields with good regioselectivity (up to $54 \%$ yield, up to $91: 9$ p:o ratio, Scheme 1$)$. Likewise, the chlorobenzene 2c reacted smoothly with propargyl $\alpha$-aryl- $\alpha$ diazoacetates $(\mathbf{1 b}, \mathbf{1 c})$ under the optimal reaction conditions to furnish the corresponding desired products (3bc, 3cc) in moderate yields with very good regioselectivity (up to $52 \%$ yield, up to $90: 10$ p:o ratio, Scheme 1).

Later, we planned to explore the reactivity of electron rich arenes. Mildly activated arene such as toluene $\mathbf{2 d}$ reacted with propargyl $\alpha$-aryl- $\alpha$-diazoacetates (1a-1b) under the optimal reaction conditions to afford the corresponding desired products (3ad-3bd) in good yields with good regioselectivity (up to $84 \%$ yield, up to 80:20 p:o ratio, Scheme 1). o-Xylene $2 \mathrm{e}$ and $p$ xylene $\mathbf{2 f}$ reacted smoothly with propargyl $\alpha$-aryl- $\alpha$-diazoacetates (1a-1b) under the optimal reaction conditions to afford the corresponding desired products (3ae-3be, 3af-3bf) in good to excellent yields (up to $94 \%$ yield, Scheme 1). Mesitylene (2g), 
when treated with propargyl $\alpha$-aryl- $\alpha$-diazoacetates (1a-1d) under optimal reaction condition, furnished the corresponding products (3ag-3dg) in excellent yields (up to $98 \%$ yield, Scheme 1). Under the optimal reaction conditions, the activated arene such as anisole $2 \mathrm{~h}$ reacted smoothly with the propargyl $\alpha$-aryl- $\alpha$ diazoacetate $\mathbf{1 b}$ to afford the corresponding desired product $\mathbf{3 b h}$ in very good yield. Activated arene such as 1,4dimethoxybenzene $2 \mathbf{i}$ reacted with different propargyl $\alpha$-aryl- $\alpha$ diazoacetates $(\mathbf{1 a}-\mathbf{1 d}, \mathbf{1 g})$ to afford the corresponding desired products (3ai-3di, 3gi) in excellent yields (up to $96 \%$, Scheme 1) under the optimized reaction conditions. Highly activated arene such as 1,3,5-trimethoxybenzene $2 \mathbf{j}$ reacted smoothly with propargyl $\alpha$-aryl- $\alpha$-diazoacetates $(\mathbf{1 a}, \mathbf{1 b}, \mathbf{1 g})$ to furnish the corresponding $\mathrm{C}-\mathrm{H}$ bond functionalization products (3aj, $\mathbf{3 b j}$, $3 \mathbf{g j}$ ) in very good to excellent yields (up to $92 \%$, Scheme 1). 1,3Benzodioxole $\mathbf{2 k}$ also reacted smoothly with various propargyl $\alpha$ aryl- $\alpha$-diazoacetates $(\mathbf{1} \mathbf{a}, \mathbf{1} \mathbf{b}, \mathbf{1} \mathbf{h}, \mathbf{1 i})$ under the optimal reaction conditions to afford the corresponding desired $\mathrm{C}-\mathrm{H}$ functionalized products (3ak, $3 \mathbf{b k}, 3 \mathbf{h k}$, 3ik) in moderate to excellent yields with excellent regioselectivity (up to $94 \%$ yield, up to $99: 1$ p:o ratio, Scheme 1).

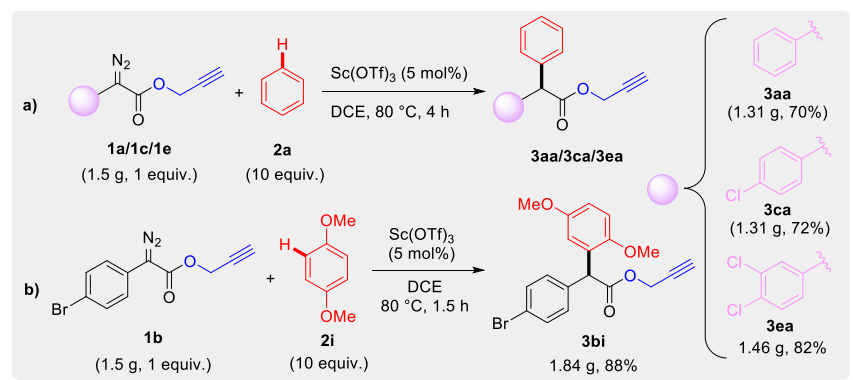

Scheme 2. Gram-scale synthesis of $\alpha$-diarylacetates

It is significant to note that $\mathrm{C}-\mathrm{H}$ bond functionalization of 1,3 benzodioxole $2 \mathbf{k}$ worked smoothly with $\alpha$-(4-methoxyphenyl)- $\alpha$ diazoacetate 1i, unlike the previous procedure. ${ }^{[5 e]}$ Even the heteroaromatic arene such as 2,5-dimethylfuran $\mathbf{2 I}$, when treated with propargyl $\alpha$-aryl- $\alpha$-diazoacetate $\mathbf{1 b}$ under the optimal reaction conditions afforded the $\mathrm{C}-\mathrm{H}$ functionalization product $3 \mathbf{b l}$ in $60 \%$ yield (see Scheme 1). We demonstrated the robustness of this protocol using different arenes and propargyl $\alpha$-aryl- $\alpha$-diazoacetates. It is also very significant to note that
To demonstrate the practicality and the generality of the protocol, we further explored this protocol for the gram scale synthesis of few $\alpha$-diarylacetates. Reactions of propargyl $\alpha$-aryl- $\alpha$ diazoacetates (1a, 1c, 1e, and $\mathbf{1 b})$ and arenes (2a and $\mathbf{2 i})$ on a gram scale afforded the corresponding $\alpha$-diarylacetates (3aa, 3ca, 3ea, 3bi) in very good yields under the optimal reaction conditions (up to $88 \%$ yield, Scheme 2). The protocol proved to be very effective on a gram scale and found to be reproducible. Later, the hydrolysis of propargyl $\alpha$-diarylacetates (3aa, 3ca, 3ea) was carried out using $\mathrm{LiOH} . \mathrm{H}_{2} \mathrm{O}$ (2.5 equiv.) in ethanoltetrahydrofuran-water $(1: 1: 1)$ mixture at room temperature in $1 \mathrm{~h}$, to furnish the corresponding $\alpha$-diarylacids (4aa, 4ca, and 4ea) in excellent yields (up to $96 \%$ yield, Scheme 3 ). The easy removal of propargyl group under mild condition proved to be useful for the further synthetic transformations.

Further, in order to demonstrate the applicability of this protocol, we explored the synthesis of biologically active adiphenine $\mathbf{2 1}$ on a gram scale. Adiphenine is a nicotinic receptor inhibitor, used as an antispasmodic drug ${ }^{[12]}$ The $\mathrm{C}-\mathrm{H}$ bond functionalization of benzene 2a with propargyl $\alpha$-phenyl- $\alpha$-diazoacetate $1 \mathrm{a}$ in the presence of catalytic amount of Sc(OTf) 3 (5 mol\%) afforded the corresponding product 3aa. This was further utilized for the synthesis of adiphenine 4 in two steps in good yield ( $85 \%$, Scheme 4).

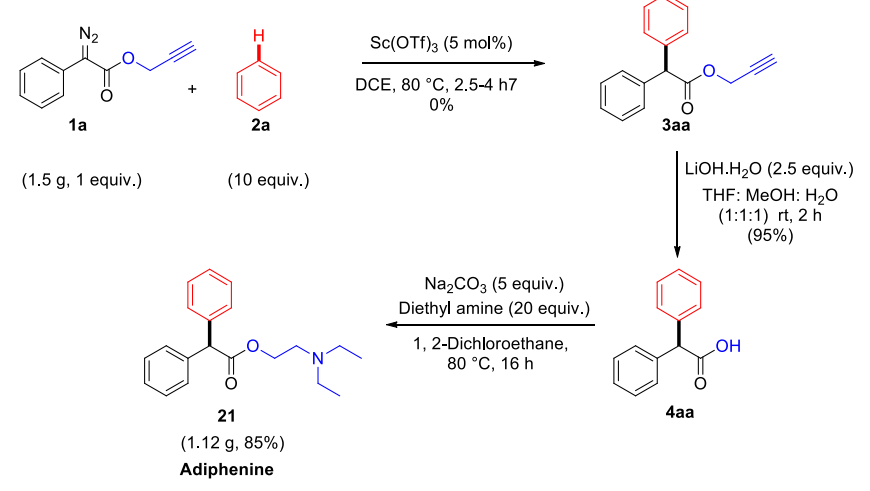

Scheme 4. Gram-scale synthesis of Adiphenine-An antispasmodic drug

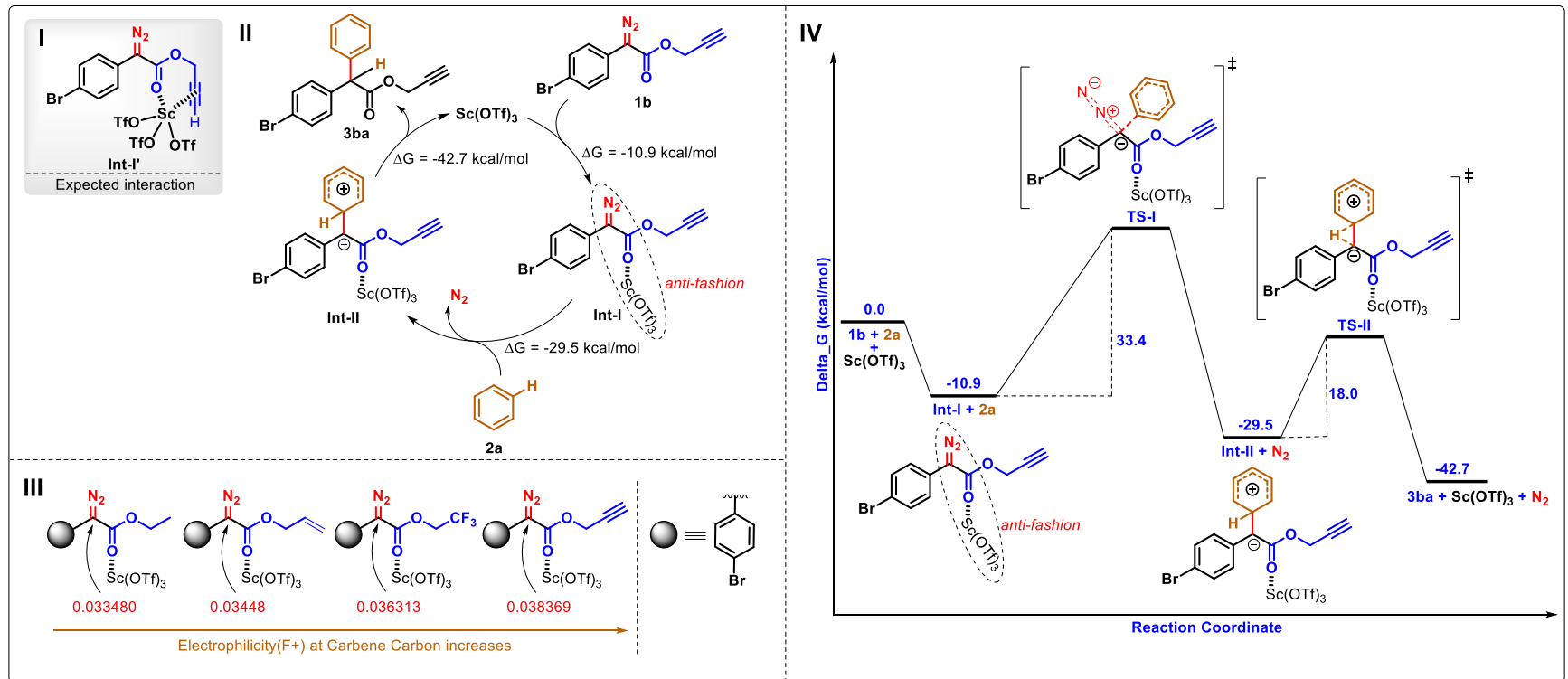

Figure. 2: Mechanistic investigation. I: Expected interaction of scandium metal with alkyne. II: Possible mechanism for the C-H bond functionalization of benzene under Scandiumcatalysis. III: Electrophilicity measurement employing Fukui function indices. IV: The energy profile for the reaction of propargyl -(4-bromophenyl)- -diazoacetate with benzene, with the scandium triflate $\left[\mathrm{Sc}(\mathrm{OTf})_{3}\right]$ catalyst. Energies are in $\mathrm{kcal} / \mathrm{mol}$.

substrates containing $\mathrm{C}\left(\mathrm{sp}^{3}\right)-\mathrm{H}$ bonds (benzylic $\mathrm{C}-\mathrm{H}$ ) underwent chemo-selective arene $\mathrm{C}-\mathrm{H}$ bond functionalization exclusively. [13b-13d]
After successfully demonstrating the practicability of the protocol, we further planned to understand the reaction pathway. In order to understand whether or not the $\mathrm{C}-\mathrm{H}$ bond cleavage of benzene is a rate-determining step in this reaction, we carried 
out kinetic isotope effect studies. The studies indicated that cleavage of the $\mathrm{C}-\mathrm{H}$ bond of benzene was not a ratedetermining step $\left(K_{H} / K_{D}=1\right.$, see Appendix III, ESI). Further, to confirm whether or not the direct $[1,2]-\mathrm{H}$ shift from benzene takes place in this transformation, we carried out another controlled experiment (see Appendix III, ESI). This result indicated that $48 \%$ of direct $[1,2]-D$ shift from the deuterated benzene occurred in this transformation. The third controlled experiment in $\mathrm{D}_{2} \mathrm{O}$ (5 equiv.) indicated that there was no direct proton exchange of 3 ba with $\mathrm{D}_{2} \mathrm{O}$ under the optimized reaction conditions (see Appendix III, ESI).

In order to have further insight into the mechanism, computational calculations have been performed in detail using density functional theory (DFT) at the PBE/TZVP level of theory. We also calculated the Fukui function indices to investigate the electrophilicity of the carbon where the diazo moiety (carbene precursor) is attached. The turn over frequency (TOF) has also been calculated to compare the activity for different groups associated with the ester carbon of $\alpha$-aryl- $\alpha$-diazoacetates.

Based on the experimental results, previous literature reports ${ }^{[15]}$ and quantum chemical calculations that we have carried out the plausible catalytic cycle has been depicted in Figure 2.

At first the Hirshfield population of carbene carbon of different alkyl $\alpha$-( $p$-bromophenyl)- $\alpha$-diazoacetates has been calculated to get insight on the electrophilic diazoacetate. Based on the Fukui function indices, the diazo carbon of propargyl $\alpha$ - $(p$ bromophenyl)- $\alpha$-diazoacetate was found to be the most electrophilic $\left(\mathrm{F}^{+}=0.064785\right)$. As this preliminary result attained our hypothesis, we further sought to optimize the interaction of propargyl $\alpha$-aryl- $\alpha$-diazoacetate with $\mathrm{Sc}(\mathrm{OTf})_{3}$ to investigate the most stable conformation. We optimized different interactions of $\mathrm{Sc}(\mathrm{OTf})_{3}$ with ester oxygen(s) and acetylenic moiety of the propargyl group $\alpha$-aryl- $\alpha$-diazoacetate (see ESI, Appendix-IV, Figure-1). We have also looked into the all possible modes of interactions of $\mathrm{Sc}(\mathrm{OTf})_{3}$ with the propargyl ester moiety. It is proposed that propargyl $\alpha$-(p-bromophenyl)- $\alpha$-diazoacetate $\mathbf{1 b}$ interacts with scandium triflate to form an intermediate int-I (scandium triflate-coordinated complex) via an energetically favourable anti-fashion $(\Delta \mathrm{G}=-10.9 \mathrm{kcal} / \mathrm{mol})$ as shown in Fig. 2 (II and IV). This intermediate int-I further reacts with benzene at an elevated temperature $\left(80{ }^{\circ} \mathrm{C}\right)$ to furnish an energetically feasible $(\Delta \mathrm{G}=-29.5 \mathrm{kcal} / \mathrm{mol})$ zwitterionic intermediate int-Il via high energy transition state TS-I $(\Delta \mathrm{G}=33.4 \mathrm{kcal} / \mathrm{mol})$. This step found to be highly favourable by the extrusion of thermodynamically stable dinitrogen (Fig. 2 II and IV). The intermediate int-II finally affords the highly stable product 3 ba $(\Delta \mathrm{G}=-42.7 \mathrm{kcal} / \mathrm{mol})$ via the transition state TS-II $(\Delta \mathrm{G}=18.0$ $\mathrm{kcal} / \mathrm{mol}$ ) by the abstraction of proton and regenerating the catalyst.

Further we calculated the Hirshfield population of carbene carbon of different Sc(OTf) 3 -bounded alkyl $\alpha$-(p-bromophenyl)- $\alpha$ diazoacetates. Based on the Fukui function indices, the diazo carbon of propargyl $\alpha$-( $p$-bromophenyl)- $\alpha$-diazoacetate-Sc(OTf) complex was the most electrophilic $\left(\mathrm{F}^{+}=0.038369\right.$ ) (for more details, see ESI, Appendix-IV). This result unambiguously supports that propargyl moiety on $\alpha$-aryl- $\alpha$-diazoester enhances the electrophilicity of the diazocarbon and thus significantly increases the reactivity towards the nucleophilic arenes. This computational result is in accordance with the experimental result wherein we observed that propargyl $\alpha$-(p-bromophenyl)- $\alpha$ diazoacetate reacted smoothly affording the desired product $\mathbf{3 b a}$ in higher yield than that of trifluorethyl $\alpha$-( $p$-bromophenyl)- $\alpha$ diazoacetate (see Table 1, entries 11 and 14 for comparison). Thermodynamics and kinetics calculations have also been calculated for the other groups that have been experimentally studied with the $\mathrm{Sc}(\mathrm{OTf})_{3}$ catalyst. The turnover frequency (TOF) has also been calculated and compared for all the energy profiles associated with the different groups (see ESI, AppendixIV). All these results are in accordance with the experimental observations. The TOF calculations suggest that the reaction of propargyl $\alpha$-aryl- $\alpha$-diazoacetates with $\mathrm{Sc}(\mathrm{OTf})_{3}$ will give the product with highest efficiency. This shows that there is a definite role of the propargyl group in activating the arene efficiently.

In conclusions, we have developed novel scandium catalyzed chemo highly- and regioselective $\mathrm{C}-\mathrm{H}$ bond functionalization of unactivated arenes and mildly deactivated arenes with propargyl $\alpha$-aryl- $\alpha$-diazoacetates as a new reagent. The combination of catalyst-Sc(OTf) 3 and reagent-propargyl $\alpha$ aryl- $\alpha$-diazoacetate worked synergistically and this was further evident from the computational studies. The practicability of this protocol demonstrated on a gram scale. The protocol has been successfully applied for the gram scale synthesis of biologically active adiphenine an antispasmodic drug.

\section{Acknowledgements}

R. G. B. thanks DST-SERB (CRG/2019/005753) and (EMR/2015/000909), New Delhi, Govt. of India for the generous research grant. R. G. B., B. S. N and D. L. also thank IISER Pune for the financial assistance. B. S. N. and S. B. thank CSIR and D. L. thanks UGC, New Delhi, Government of India for providing fellowship. S. B. and K. V. acknowledge the MultiScale simulation and Modeling project-MSM and PARAM Brahma for the computational facilities. K. V. thanks DST-SERB (EMR/2014/000013) for providing financial assistance.

\section{Corresponding Author}

Ramakrishna G. Bhat-ORCID: 0000-0002-7739-9553

\section{Authors}

Balu S. Navale

Debasish Laha

Subhrashis Banerjee

Kumar Vanka-ORCID:0000-0001-7301-7573

\section{Conflicts of interest}

There are no conflicts to declare.

\section{References}

[1] a) X. Ribas, D. Ma, J. J. Spivey, A. Casitas, I. J. S. Fairlamb, A. N. Vedernikov, L. Mirica, $\mathrm{C}-\mathrm{H}$ and $\mathrm{C}-\mathrm{X}$ Bond Functionalization: Transition Metal Mediation; Royal Society of Chemistry: Cambridge, U.K., 2013; b) C. J. Li, From $\mathrm{C}-\mathrm{H}$ to $\mathrm{C}-\mathrm{C}$ bonds: Cross-Dehydrogenative-Coupling; Royal Society of Chemistry: Cambridge, U.K., 2015; c) B.-J. Li, Z.-J. Shi, Homogeneous Catalysis for Unreactive Bond Activation; Z.-J. Shi, Ed.; John Wiley \& Sons: NJ, 2015; pp 498-521; d) J. J. Li, C-H Bond Activation in Organic Synthesis; CRC Press: Boca Raton, FL, 2015

[2] D. Lednicer, Strategies for Organic Drug Synthesis and Design; Wiley: Hoboken, 2008; pp 43-88.

[3] a) M. P. Doyle, M. A. McKervey, T. Ye, Modern Catalytic Methods for Organic Synthesis with Diazo Compounds; John Wiley \& Sons: New York, 1998; b) A. Ford, H. Miel, A. Ring, C. N. Slattery, A. R. Maguire, M. A. McKervey, Chem. Rev. 2015, 115, 9981; c) L. Liu, J. Zhang, Chem. Soc. Rev. 2016, 45, 506; d) Z. Zhang, J. Wang, Tetrahedron 2008, 64, 6577 .

[4] a) M. J. Rosenfeld, B. K. Shankar, H. Shechter, J. Org. Chem. 1988, 53, 2699; b) M. Yang, T. R. Webb, P. Livant, J. Org. Chem. 2001, 66, 4945; c) C. P. Park, A. Nagle, C. H. Yoon, C. Chen, K. W. Jung, J. Org. Chem. 2009, 74, 6231; d) A. DeAngelis, V. W. Shurtleff, O. Dmitrenko, J. M. Fox, J. Am. Chem. Soc. 2011, 133, 1650; e) W.-W. Chan, S.-F. Lo, Z. Zhou, W.-Y. Yu, J. Am. Chem. Soc. 2012, 134, 13565; f) T. K. Hyster, K. E. Ruhl, T. Rovis, J. Am. Chem. Soc. 2013, 135, 5364; g) D. Best, D. J. Burns, H. W. Lam, Angew. Chem., Int. Ed. 2015, 54, 7410; h) D. Best, M. Jean, P. van de Weghe, J. Org. Chem. 2016, 81, 7760; i) A. Manchoju, S. V. Pansare, Org. Lett. 2016, 18, 5952; j) B. Xu, M.-L. Li, X.-D. Zuo, S.-F. Zhu, Q.-L. Zhou, J. Am. Chem. Soc. 2015, 137, 8700.

[5] a) M. R. Fructos, T. R. Belderrain, P. de Fremont, N. M. Scott, S. P. Nolan, M. M. Díaz-Requejo, P. J. Ṕerez, Angew. Chem., Int. Ed. 2005, 
44, 5284; b) I. Rivilla, B. P. Gõmez-Emeterio, M. R. Fructos, M. M. Díaz-Requejo, P. J. Perez, Organometallics 2011, 30, 2855; c) P. J. Ṕerez, M. M. Díaz-Requejo, I. Rivilla, Beilstein J. Org. Chem. 2011, 7, 653; d) Z. Yu, B. Ma, M. Chen, H.-H. Wu, L. Liu, J. Zhang, J. Am. Chem. Soc. 2014, 136, 6904; e) Y. Xi, Y. Su, Z. Yu, B. Dong, E. J. McClain, Y Lan, X. Shi, Angew. Chem., Int. Ed. 2014, 53, 9817; f) B. Ma, Z. Chu, B. Huang, Z. Liu, L. Liu, J. Zhang, Angew. Chem., Int. Ed. 2017, 56, 2749 g) B. Ma, J. Wu, L. Liu, J. Zhang, Chem. Commun. 2017, 53, 10164; h) G. Xu, K. Liu, J. Sun, Org. Lett. 2018, 20, 72; i) W. Zhang, G. Xu, L. Qiu, J. Sun, Org. Biomol. Chem. 2018, 16, 3889

[6] E. Tayama, T. Yanaki, H. Iwamoto, E. Hasegawa, Eur. J. Org. Chem. 2010, 6719 .

[7] a) H. M. Mbuvi, L. K. Woo, Organometallics 2008, 27, 637; b) J.-M. Yang, Y. Cai, S.-F. Zhu, Q.-L. Zhou, Org. Biomol. Chem. 2016, 14, 5516.

[8] A. Conde, G. Sabenya, M. Rodríguez, V. Postils, J. M. Luis, M. M. Díaz-Requejo, M. Costas, P. J. Perez, Angew. Chem., Int. Ed. 2016, 55, 6530.

[9] a) T. J. Auvil, S. S. So, A. E. Mattson, Angew. Chem., Int. Ed. 2013, 52 11317; b) C. Zhai, D. Xing, C. Jing, J. Zhou, C. Wang, D. Wang, W. Hu, Org. Lett. 2014, 16, 2934; c) Z. Yu, Y. Li, J. Shi, B. Ma, L. Liu, J. Zhang, Angew. Chem., Int. Ed. 2016, 55, 14807; d) R. D. C. Gallo, P. B. Momo, D. P. Day, A. C. B. Burtoloso, Org. Lett. 2020, 22, 2339-2343.

[10] a) T. J. Cheeseright, J. L. Melville, M. Gottert, M. Holm, S. Laufer, F. Lehmann, S. Luik, J. Med. Chem. 2009, 52, 4200; b) J. Zhao, X. Fan, S Wang, S. Li, S. Shang, Y. Yang, N. Xu, Y. Lv, J. Shi, J. Nat. Prod. 2004 67, 1032; c) G. Autore, A. Caruso, S. Marzocco, B. Nicolaus, C Palladino, A. Pinto, A. Popolo, M. S. Sinicropi, G. Tommonaro, C. Saturnino, Molecules 2010, 15, 2028; d) P. Urbani, M. G. Cascio, A Ramunno, T. Bisogno, C. Saturnino, V. D. Marzo, Bioorg. Med. Chem. 2008, 16, 7510

[11] a) Y.-T. Tsoi, Z. Zhou, W.-Y. Yu, Org. Lett. 2011, 13, 5370; b) Y. Xia, Z Liu, S. Feng, F. Ye, Y. Zhang, J. Wang, Org. Lett. 2015, 17, 956; c) J. Ghorai, P. Anbarasan, J. Org. Chem. 2015, 80, 3455; d) F. Ye, S. Qu, L. Zhou, C. Peng, C. Wang, J. Cheng, M. L. Hossain, Y. Liu, Y. Zhang, Z.X. Wang, J. Wang, J. Am. Chem. Soc. 2015, 137, 4435.

[12] G. Spitzmaul, F. Gumilar, J. P. Dilger, C. Bouzat, Br. J. Pharmacol. 2009, 157, 804.

[13] a) K. King, H. Vong, S. Maeda, K. Tanaka, Chem. Eur. J. 2016, 22, 18865 (It is speculated that in addition to being weakly electronwithdrawing, the propargyl moiety may also play a previously undescribed role that accelerates aminolysis); b) H. M. L. Davies, Q. Jin P. Ren, A. Y. Kovalevsky, J. Org. Chem. 2002, 67, 4165; c) H. M. L. Davies, Q. Jin, Tetrahedron: Asymmetry 2003, 14, 941; d) D. M. Guptill, H. M. L. Davies, J. Am. Chem. Soc. 2014, 136, 17718 .

[14] CCDC number of compound 3aa is 1848210. The number contains all crystallographic details of this publication and is available free of charge at https://www.ccdc.cam.ac.uk/structures/

[15] W. Li, X. Liu, X. Hao, X. Hu, Y. Chu, W. Cao, S. Qin, C. Hu, L. Lin, X Feng, J. Am. Chem. Soc. 2011, 133, 15268-15271. 


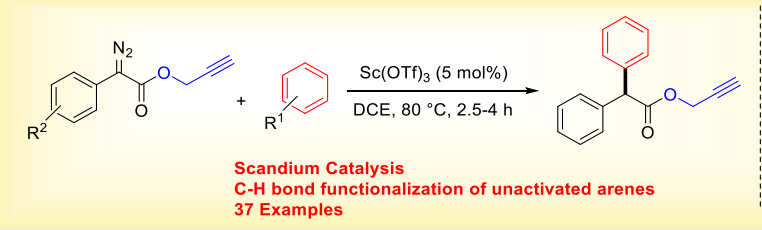

Balu S. Navale; Debasish Laha, Subhrashis Banerjee, Kumar Vanka and Ramakrishna G. Bhat ${ }^{\star}$

Page No. - Page No.

Highly Site-Selective Direct C-H Bond Functionalization of Unactivated Arenes with Propargyl $\alpha$-Aryl- $\alpha$-diazoacetates via Scandium Catalysis 


\section{Highly Site-Selective Direct $\mathbf{C}-\mathbf{H}$ Bond Functionalization of Unactivated Arenes with Propargyl $\alpha$-Aryl- $\alpha$-diazoacetates via Scandium Catalysis}

Balu S. Navale, ${ }^{a}$ Debasish Laha, ${ }^{\text {a }}$ Subhrashis Banerjee, ${ }^{\text {b,c }}$ Kumar Vanka, ${ }^{\text {,cc }}$ and Ramakrishna G. Bhat ${ }^{\text {a* }}$

rgb@iiserpune.ac.in

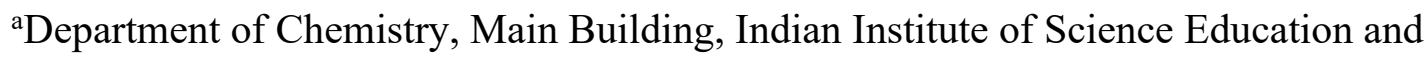
Research (IISER)-Pune, Dr. Homi Bhabha Road, Pashan, Pune, 411008, Maharashtra, India; ${ }^{b}$ Physical and Materials Chemistry Division, CSIR-National Chemical Laboratory, Pune-

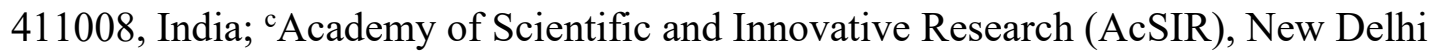

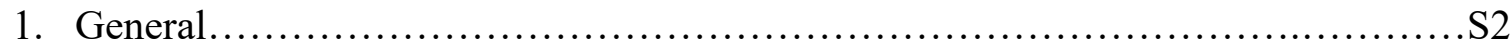

2. General procedure A for the synthesis of Propargyl esters...................................S2-S6

3. General procedure B for the synthesis of $\alpha$-aryl- $\alpha$ diazoacetates.......................S7-S12

4. General Procedure $\mathrm{C} 1$ for optimization of reaction conditions................... 12

5. General Procedure C2 for screening of solvents and Appendix-I................S13

6. General procedure C3 for screening of co-catalyst and Appendix-II...............S14

7. General procedure D for synthesis of $\alpha$-diarylacetates.....................S14-S34

8. General procedure E for gram-scale synthrsis of $\alpha$-diarylacetates...................S35-S37

9. General procedure $\mathrm{F}$ for the deprotection of propargyl group........................S37-S38

10. Procedure for gram-scale synthesis of Adiphenine: antispasmodic drug........S39-S40

11. Mechanistic Investigations

Appendix-III (studies of kinetic isotopic effect).....................S40-S41

Appendix-IV (Computational studies).............................S42-S49

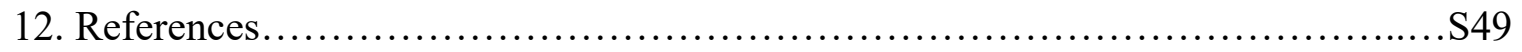

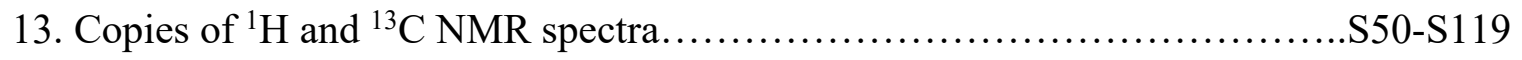

14. Crystal data of compound 3aa................................................................. $120-\mathrm{S} 121$ 


\section{General}

Unless otherwise noted, all reactions were carried out with distilled and dried solvents using oven-dried glassware. All the reagents were purchased from commercial sources and used as received unless otherwise indicated. Thin-layer chromatography (TLC) was performed using silica gel $60 \mathrm{GF}_{254}$ precoated aluminum backed plates $(2.5 \mathrm{~mm})$ with detection by UV light. ${ }^{1} \mathrm{H}$ NMR and ${ }^{13} \mathrm{C}$ NMR spectra were recorded in $\mathrm{CDCl}_{3}$ and DMSO$\mathrm{d}_{6}$. Chemical shifts in ${ }^{1} \mathrm{H}$ NMR spectra are reported as $\delta$ in units of parts per million (ppm) downfield from tetramethylsilane with the solvent resonance as the internal standard or from the residual solvent peak as internal standard and $J$ values are given in $\mathrm{Hz} .{ }^{13} \mathrm{C}$ NMR spectra are reported as $\delta$ in ppm downfield from tetramethylsilane and relative to the signal of chloroform-d and DMSO- $\mathrm{d}_{6} .{ }^{13} \mathrm{C}$ NMR spectra were recorded with complete proton decoupling. Mass samples were analyzed by high-resolution mass spectrometry (HRMS) using ESI TOF. FT-IR spectra were obtained using a FT-IR spectrophotometer as neat and reported in $\mathrm{cm}^{-1}$. Melting points were measured in an open glass capillary and values are uncorrected.

\section{General procedure A for the synthesis of propargyl esters}

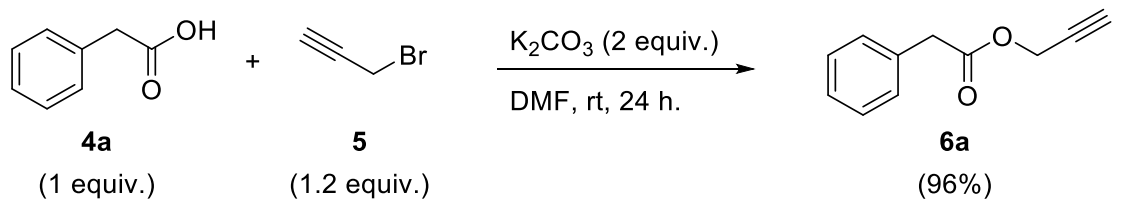

To the stirred solution of 2-phenylacetic acid 4a $(2.72 \mathrm{~g}, 20 \mathrm{mmol})$ in DMF $(15 \mathrm{~mL})$ was added propargyl bromide solution $80 \%$ in toluene $(3.57 \mathrm{~g}, 24 \mathrm{mmol})$ and $\mathrm{K}_{2} \mathrm{CO}_{3}(5.53 \mathrm{~g}, 40$ mmol). The reaction mixture was stirred at room temperature for $24 \mathrm{~h}$. The progress of the reaction was monitored by TLC. The reaction mixture was filtered through celite, and the filtrate was diluted with water $(30 \mathrm{~mL})$ and extracted with ethyl acetate $(3 \times 30 \mathrm{~mL})$. The combined organic layers were washed with cold water and brine solution. The extract was dried over anhydrous $\mathrm{Na}_{2} \mathrm{SO}_{4}$ and filtered. The filtrate was evaporated under vacuum and the crude product was purified using column chromatography over silica gel to afford product 6a as colourless liquid ( $3.34 \mathrm{~g}, 96 \%$ yield). ${ }^{1}$

\section{Prop-2-yn-1-yl 2-phenylacetate (6a):}


if $\pi_{0}^{0}=$

Compound 6a was synthesized following the general procedure (A). The product was obtained as pale yellow liquid (3.34 g, 96\% yield): $R_{f}=0.35$ petroleum ether/EtOAc (95:5); IR (neat) $\mathrm{cm}^{-1}: 3288,2946,2128,1737,1605,1496,1446,1333,1239,1136,999,938,760$, 697; ${ }^{1} \mathrm{H}$ NMR (400 MHz, $\left.\mathrm{CDCl}_{3}\right): \delta 7.42-7.19(\mathrm{~m}, 5 \mathrm{H}), 4.69(\mathrm{~d}, J=2.5 \mathrm{~Hz}, 2 \mathrm{H}), 3.68(\mathrm{~s}$, 2H), $2.47(\mathrm{t}, J=2.5 \mathrm{~Hz}, 1 \mathrm{H}) ;{ }^{13} \mathrm{C} \mathrm{NMR}\left(100 \mathrm{MHz}, \mathrm{CDCl}_{3}\right): \delta 170.9,133.5,129.4,128.8$, 127.4, 77.6 , 75.2, 52.5, 41.1; HRMS (ESI TOF): Calculated for $\mathrm{C}_{11} \mathrm{H}_{10} \mathrm{NaO}_{2}(\mathrm{M}+\mathrm{Na})^{+}$: 197.0578, Found: 197.0576.

\section{Prop-2-yn-1-yl 2-(4-bromophenyl)acetate (6b):}<smiles>C#CCOC(=O)Cc1ccc(Br)cc1</smiles>

Compound $\mathbf{6 b}$ was synthesized following the general procedure (A). The product was obtained as white solid (4.657 g, 92\% yield): $R_{f}=0.35$ petroleum ether/EtOAc (95:5); m.p.: 41-43 ${ }^{\circ} \mathrm{C}$; IR (neat) $\mathrm{cm}^{-1}: 3288,3032,2946,2128,1737,1605,1239,1136,831,760,697$, 639; ${ }^{1} \mathrm{H}$ NMR (400 MHz, $\left.\mathrm{CDCl}_{3}\right): \delta 7.46(\mathrm{~d}, J=8.4 \mathrm{~Hz}, 2 \mathrm{H}), 7.17(\mathrm{~d}, J=8.4 \mathrm{~Hz}, 2 \mathrm{H}), 4.70$ (d, $J=2.5 \mathrm{~Hz}, 2 \mathrm{H}), 3.63$ (s, 2H), 2.48 (t, $J=2.5 \mathrm{~Hz}, 1 \mathrm{H}) ;{ }^{13} \mathrm{C}$ NMR (100 MHz, DMSO-d 6 ): $\delta$ 170.4, 131.9, 131.2, 129.7, 121.5, 77.4, 75.3, 52.6, 40.4; HRMS (ESI TOF): Calculated for $\mathrm{C}_{11} \mathrm{H}_{10} \mathrm{BrO}_{2}(\mathrm{M}+\mathrm{H})^{+}$: 252.9864, Found: 252.9871.

\section{Prop-2-yn-1-yl 2-(4-chlorophenyl)acetate (6c):}<smiles>C#CCOC(=O)Cc1ccc(Cl)cc1</smiles>

Compound $\mathbf{6 c}$ was synthesized following the general procedure (A). The product was obtained as viscous liquid (3.964 g, 95\% yield): $R_{f}=0.3$ petroleum ether/EtOAc (95:5); IR (neat) $\mathrm{cm}^{-1}: 3293,2948,2129,1738,1597,1492,1371,1143,1091,938,807,758,676,641$; ${ }^{1} \mathrm{H}$ NMR (400 MHz, DMSO-d 6 ): $\delta 7.41-7.36$ (m, 2H), $7.33-7.27$ (m, 2H), 4.72 (d, $J=2.5$ $\mathrm{Hz}, 2 \mathrm{H}), 3.75$ (s, 2H), 3.55 (t, $J=2.5 \mathrm{~Hz}, 1 \mathrm{H}) ;{ }^{13} \mathrm{C}$ NMR (100 MHz, DMSO-d $\left.\mathrm{d}_{6}\right): \delta 170.1$, 133.0, 131.6, 131.2, 128.2, 78.2, 77.7, 52.1, 39.0; HRMS (ESI TOF): Calculated for $\mathrm{C}_{11} \mathrm{H}_{10} \mathrm{ClO}_{2}(\mathrm{M}+\mathrm{H})^{+}:$209.0369, Found: 209.0365. 


\section{Prop-2-yn-1-yl 2-(4-fluorophenyl)acetate (6d):}<smiles>C#CCOC(=O)Cc1ccc(F)cc1</smiles>

Compound 6d was synthesized following the general procedure (A). The product was obtained as colourless liquid (3.611 g, 94\% yield): $R_{f}=0.35$ petroleum ether/EtOAc (95:5); IR (neat) $\mathrm{cm}^{-1}: 3295,2948,2129,1739,1606,1510,1222,1140,937,826,785,681,643 ;{ }^{1} \mathrm{H}$ NMR (400 MHz, DMSO-d 6 ): $\delta 7.38-7.28(\mathrm{~m}, 2 \mathrm{H}), 7.20-7.11(\mathrm{~m}, 2 \mathrm{H}), 4.71(\mathrm{~d}, J=2.5 \mathrm{~Hz}$, 2H), $3.73(\mathrm{~s}, 2 \mathrm{H}), 3.54$ (t, $J=2.5 \mathrm{~Hz}, 1 \mathrm{H}) ;{ }^{13} \mathrm{C}$ NMR (100 MHz, DMSO-d 6 ): $\delta$ 170.3, 161.2 $(\mathrm{d}, J=242.8 \mathrm{~Hz}), 131.3(\mathrm{~d}, J=8.1 \mathrm{~Hz}), 130.1(\mathrm{~d}, J=3.1 \mathrm{~Hz}), 115.0(\mathrm{~d}, J=21.3 \mathrm{~Hz}), 78.3$, 77.7, 52.0, 38.8; HRMS (ESI TOF): Calculated for $\mathrm{C}_{11} \mathrm{H}_{9} \mathrm{FNaO}_{2}(\mathrm{M}+\mathrm{Na})^{+}:$215.0484, Found: 215.0490.

\section{Prop-2-yn-1-yl 2-(3,4-dichlorophenyl)acetate (6e):}<smiles>C#CCOC(=O)Cc1ccc(Cl)c(Cl)c1</smiles>

Compound 6e was synthesized following the general procedure (A). The product was obtained as colourless liquid (4.473 g, 92\% yield): $R_{f}=0.35$ petroleum ether/EtOAc (95:5); IR (neat) $\mathrm{cm}^{-1}: 3295,2948,2130,1738,1133,1026,998,877,820,781,743,637 ;{ }^{1} \mathrm{H}$ NMR $\left(400 \mathrm{MHz} \mathrm{CDCl}_{3}\right): \delta 7.44-7.35(\mathrm{~m}, 2 \mathrm{H}), 7.13(\mathrm{dd}, J=8.3,2.1 \mathrm{~Hz}, 1 \mathrm{H}), 4.71(\mathrm{~d}, J=2.5$ $\mathrm{Hz}, 2 \mathrm{H}), 3.63(\mathrm{~s}, 2 \mathrm{H}), 2.50(\mathrm{t}, J=2.5 \mathrm{~Hz}, 1 \mathrm{H}) ;{ }^{13} \mathrm{C} \mathrm{NMR}\left(100 \mathrm{MHz}, \mathrm{CDCl}_{3}\right): \delta 163.6,133.5$, 130.9, 129.8, 125.7, 125.4, 122.8, 77.4, 75.6, 52.6; HRMS (ESI TOF): Calculated for $\mathrm{C}_{11} \mathrm{H}_{9} \mathrm{Cl}_{2} \mathrm{O}_{2}(\mathrm{M}+\mathrm{H})^{+}:$242.9980, Found: 242.9984 . 


\section{Prop-2-yn-1-yl 2-(3,5-difluorophenyl)acetate (6f):}<smiles>C#CCOC(=O)Cc1cc(F)cc(F)c1</smiles>

Compound $6 \mathbf{f}$ was synthesized following the general procedure (A). The product was obtained as colourless liquid (3.909 g, 93\% yield): $R_{f}=0.35$ petroleum ether/EtOAc (95:5); IR (neat) $\mathrm{cm}^{-1}: 3299,3094,2951,2130,1741,1626,1306,1120,994,936,850,787,667$, 642; ${ }^{1} \mathrm{H}$ NMR (400 MHz, $\left.\mathrm{CDCl}_{3}\right): \delta 6.91-6.79(\mathrm{~m}, 2 \mathrm{H}), 6.75(\mathrm{~m}, 1 \mathrm{H}), 4.72(\mathrm{~d}, J=2.5 \mathrm{~Hz}$, 2H), $3.65(\mathrm{~s}, 2 \mathrm{H}), 2.50(\mathrm{t}, J=2.5 \mathrm{~Hz}, 1 \mathrm{H}) ;{ }^{13} \mathrm{C} \mathrm{NMR}\left(100 \mathrm{MHz}, \mathrm{CDCl}_{3}\right): \delta 169.7,163.1$ (dd, $J=248.7,12.9 \mathrm{~Hz}), 136.9(\mathrm{t}, J=9.8 \mathrm{~Hz}), 113.5-111.6(\mathrm{~m}), 103.1(\mathrm{t}, J=25.2 \mathrm{~Hz}), 77.3$, 75.4, 52.8, $40.6(\mathrm{t}, J=2.0 \mathrm{~Hz})$; HRMS (ESI TOF): Calculated for $\mathrm{C}_{11} \mathrm{H}_{9} \mathrm{FNaO}_{2}(\mathrm{M}+\mathrm{Na})^{+}$: 215.0484, Found: 215.0490 .

\section{Prop-2-yn-1-yl 2-(naphthalen-2-yl)acetate (6g):}<smiles>C#CCOC(=O)Cc1ccc2ccccc2c1</smiles>

Compound $\mathbf{6 g}$ was synthesized following the general procedure (A). The product was obtained as white solid (4.261 g, 95\% yield): $R_{f}=0.3$ petroleum ether/EtOAc (95:5); m.p.: $50-51^{\circ} \mathrm{C}$; IR (neat) $\mathrm{cm}^{-1}: 3288,3055,2311,2129,1736,1509,1325,1135,946,900,857$, 748, 679, 636; ${ }^{1} \mathrm{H}$ NMR (400 MHz, $\mathrm{CDCl}_{3}$ ): $\delta$ 7.82-7.78 (m, 3H), 7.74 (s, 1H), $7.54-7.29$ $(\mathrm{m}, 3 \mathrm{H}), 4.71(\mathrm{~d}, J=2.5 \mathrm{~Hz}, 2 \mathrm{H}), 3.83(\mathrm{~s}, 2 \mathrm{H}), 2.47(\mathrm{t}, J=2.5 \mathrm{~Hz}, 1 \mathrm{H}) ;{ }^{13} \mathrm{C}$ NMR $(100 \mathrm{MHz}$, $\left.\mathrm{CDCl}_{3}\right): \delta 170.8,133.6,132.7,131.0,128.5,128.2,127.83,127.80,127.4,126.3,126.0,77.7$, 75.2, 52.5, 41.3; HRMS (ESI TOF): Calculated for $\mathrm{C}_{13} \mathrm{H}_{13} \mathrm{O}_{2}(\mathrm{M}+\mathrm{H})^{+}$: 225.0916, Found: 225.0908 .

\section{Prop-2-yn-1-yl 2-(p-tolyl)acetate (6h):}<smiles>C#CCOC(=O)Cc1ccc(C)cc1</smiles>

Compound $\mathbf{6 h}$ was synthesized following the general procedure (A). The product was obtained as pale yellow liquid (3.539 g, 94\% yield): $R_{f}=0.35$ petroleum ether/EtOAc (95:5); IR (neat) $\mathrm{cm}^{-1}: 3288,2945,2129,1740,1515,1241,1141,1005,938,808,774,682,644 ;{ }^{1} \mathrm{H}$ 
NMR (400 MHz, $\left.\mathrm{CDCl}_{3}\right): \delta 7.17(\mathrm{~d}, J=8.0 \mathrm{~Hz}, 2 \mathrm{H}), 7.13(\mathrm{~d}, J=8.0 \mathrm{~Hz}, 2 \mathrm{H}), 4.68(\mathrm{~d}, J=$ $2.5 \mathrm{~Hz}, 2 \mathrm{H}), 3.63(\mathrm{~s}, 2 \mathrm{H}), 2.47(\mathrm{t}, J=2.5 \mathrm{~Hz}, 1 \mathrm{H}), 2.33(\mathrm{~s}, 3 \mathrm{H}) ;{ }^{13} \mathrm{C}$ NMR $(100 \mathrm{MHz}$, $\left.\mathrm{CDCl}_{3}\right): \delta 171.0,137.0,130.5,129.4,129.3,77.7,75.1,52.4,40.7,21.2$; HRMS (ESI TOF): Calculated for $\mathrm{C}_{12} \mathrm{H}_{13} \mathrm{O}_{2}(\mathrm{M}+\mathrm{H})^{+}$: 189.0916, Found: 189.0912.

\section{Prop-2-yn-1-yl 2-(4-methoxyphenyl)acetate (6i):}<smiles>C#CCOC(=O)Cc1ccc(OC)cc1</smiles>

Compound 6i was synthesized following the general procedure (A). The product was obtained as colourless liquid (3.88 g, $95 \%$ yield): $R_{f}=0.5$ petroleum ether/EtOAc (80:20); IR (neat) $\mathrm{cm}^{-1}: 3285,2948,2128,1736,1511,1242,1138,1025,938,819,780,682,643 ;{ }^{1} \mathrm{H}$ NMR (400 MHz, $\left.\mathrm{CDCl}_{3}\right): \delta 7.23-7.17(\mathrm{~m}, 2 \mathrm{H}), 6.89-6.83(\mathrm{~m}, 2 \mathrm{H}), 4.68(\mathrm{~d}, J=2.5 \mathrm{~Hz}$, 2H), $3.79(\mathrm{~s}, 3 \mathrm{H}), 3.61(\mathrm{~s}, 2 \mathrm{H}), 2.47(\mathrm{t}, J=2.5 \mathrm{~Hz}, 1 \mathrm{H}) ;{ }^{13} \mathrm{C}$ NMR $\left(100 \mathrm{MHz}, \mathrm{CDCl}_{3}\right): \delta$ 171.1, 159.0, 130.4, 125.6, 114.2, 77.7, 75.1, 55.4, 52.4, 40.2; HRMS (ESI TOF): Calculated for $\mathrm{C}_{12} \mathrm{H}_{12} \mathrm{NaO}_{3}(\mathrm{M}+\mathrm{Na})^{+}: 227.0684$, Found: 227.0685 .

\section{Prop-2-yn-1-yl 2-(3,5-dimethoxyphenyl)acetate (6j):}<smiles>C#CCOC(=O)Cc1cc(OC)cc(OC)c1</smiles>

Compound $\mathbf{6 j}$ was synthesized following the general procedure (A). The product was obtained as colourless liquid ( $4.263 \mathrm{~g}, 91 \%$ yield): $R_{f}=0.25$ petroleum ether/EtOAc $(90: 10)$; IR (neat) $\mathrm{cm}^{-1}: 3282,2946,2127,1739,1597,1201,1142,1061,941,835,782,683,651 ;{ }^{1} \mathrm{H}$ NMR (400 MHz, $\left.\mathrm{CDCl}_{3}\right): \delta 6.44(\mathrm{~d}, J=2.2 \mathrm{~Hz}, 2 \mathrm{H}), 6.37(\mathrm{t}, J=2.2 \mathrm{~Hz}, 1 \mathrm{H}), 4.69(\mathrm{~d}, J=2.5$ $\mathrm{Hz}, 2 \mathrm{H}), 3.77$ (s, 6H), 3.60 (s, 2H), $2.48(\mathrm{t}, J=2.5 \mathrm{~Hz}, 1 \mathrm{H}) ;{ }^{13} \mathrm{C} \mathrm{NMR}\left(100 \mathrm{MHz}, \mathrm{CDCl}_{3}\right): \delta$ 170.6, 161.0, 135.5, 107.4, 99.5, 77.6, 75.1, 55.4, 52.4, 41.3; HRMS (ESI TOF): Calculated for $\mathrm{C}_{13} \mathrm{H}_{15} \mathrm{O}_{4}(\mathrm{M}+\mathrm{H})^{+}:$235.0970, Found: 235.0971. 


\section{General procedure B for the synthesis of $\alpha$-aryl- $\alpha$-diazoacetates}<smiles>C#CCOC(=O)Cc1ccccc1</smiles>

$6 a$

(1 equiv.)
DBU ( 1.5 equiv.)

4-acetamidobenzenesulfonyl azide (1.2 equiv.)

$\mathrm{ACN}, 0^{\circ} \mathrm{C}-\mathrm{rt}, 16 \mathrm{~h}$<smiles>C#CCOC(=O)C(=N)c1ccccc1</smiles>

To the stirred solution propargyl 2-phenylacetate 6a $(1.74 \mathrm{~g}, 10 \mathrm{mmol})$ in $15 \mathrm{~mL}$, acetonitrile was added 4-acetamidobenzenesulfonyl azide (2.88 g, $12 \mathrm{mmol})$ and DBU (2.24 $\mathrm{mL}, 15 \mathrm{mmol})$ at ambient temperature under inert atmosphere. The reaction mixture was stirred for $16 \mathrm{~h}$ at room temperature. The reaction was quenched with saturated $\mathrm{NH}_{4} \mathrm{Cl}$, and the product was extracted with diethyl ether $(30 \mathrm{~mL}$ x 3). The combined organic layers were washed with brine and dried over anhydrous $\mathrm{Na}_{2} \mathrm{SO}_{4}$. The extract was filtered, and the filtrate was evaporated under vacuum. The crude product was purified using column chromatography over silica gel to afford $\alpha$-aryl- $\alpha$-diazoacetates 1a as orange solid (1.934 g, $84 \%$ yield $)^{2}$

\section{Prop-2-yn-1-yl 2-diazo-2-phenylacetate (1a):}<smiles>C#CCOC(=O)C(=N)c1ccccc1</smiles>

Compound 1a was synthesized following the general procedure (B). The product was obtained as Orange solid (1.521 g, 76\% yield): $R_{f}=0.4$ petroleum ether/EtOAc (95:5); m.p.: 41-43 ${ }^{\circ} \mathrm{C}$; IR (neat) $\mathrm{cm}^{-1}: 3295,3062,2948,2085,1696,1597,1497,1240,1143,1017,950$, 752, 682, 637; ${ }^{1} \mathrm{H}$ NMR (400 MHz, $\left.\mathrm{CDCl}_{3}\right): \delta 7.50$ - $7.46(\mathrm{~m}, 2 \mathrm{H}), 7.44-7.36(\mathrm{~m}, 2 \mathrm{H}), 7.24$ - $7.15(\mathrm{~m}, 1 \mathrm{H}), 4.88(\mathrm{~d}, J=2.5 \mathrm{~Hz}, 2 \mathrm{H}), 2.52(\mathrm{t}, J=2.5 \mathrm{~Hz}, 1 \mathrm{H}) ;{ }^{13} \mathrm{C}$ NMR $(100 \mathrm{MHz}$, $\mathrm{CDCl}_{3}$ ): $\delta 164.4,129.1,126.2,125.1,124.1,77.7,75.3,52.3$; HRMS (ESI TOF): Calculated for $\mathrm{C}_{11} \mathrm{H}_{8} \mathrm{~N}_{2} \mathrm{NaO}_{2}(\mathrm{M}+\mathrm{Na})^{+}:$223.0483, Found: 223.0478 . 


\section{Ethyl 2-diazo-2-phenylacetate (1a'):}<smiles>CCOC(=O)C(=N)c1ccccc1</smiles>

Compound 1a' was synthesized following the general procedure (B). The product was obtained as orange liquid (1.522 $\mathrm{g}, 80 \%$ yield): $R_{f}=0.35$ petroleum ether/EtOAc (95:5); IR (neat) $\mathrm{cm}^{-1}: 2984,2081,1696,1597,1496,1387,1286,1156,1094,979,823,688 ;{ }^{1} \mathrm{H}$ NMR $\left(400 \mathrm{MHz}, \mathrm{CDCl}_{3}\right): \delta 7.51-7.45(\mathrm{~m}, 2 \mathrm{H}), 7.41-7.35(\mathrm{~m}, 2 \mathrm{H}), 7.20-7.15(\mathrm{~m}, 1 \mathrm{H}), 4.33(\mathrm{q}$, $J=7.2 \mathrm{~Hz}, 2 \mathrm{H}), 1.34(\mathrm{t}, J=7.2 \mathrm{~Hz}, 3 \mathrm{H}) ;{ }^{13} \mathrm{C} \mathrm{NMR}\left(100 \mathrm{MHz}, \mathrm{CDCl}_{3}\right): \delta 165.4,129.0,125.9$, 125.8, 124.1, 61.1, 14.6; HRMS (ESI TOF): Calculated for $\mathrm{C}_{10} \mathrm{H}_{10} \mathrm{~N}_{2} \mathrm{NaO}_{2}(\mathrm{M}+\mathrm{Na})^{+}$: 213.0639, Found: 213.0631.

\section{Allyl 2-diazo-2-phenylacetate (1a'):}<smiles>C=CCOC(=O)C(=N)c1ccccc1</smiles>

Compound 1a" was synthesized following the general procedure (B). The product was obtained as orange liquid (1.516 g, 75\% yield): $R_{f}=0.35$ petroleum ether/EtOAc (95:5); IR (neat) $\mathrm{cm}^{-1}: 3028,2952,2085,1700,1598,1497,1242,1151,1043,1012,929,753,694$; ${ }^{1} \mathrm{H}$ NMR (400 MHz, $\left.\mathrm{CDCl}_{3}\right): \delta$ 7.50-7.47 (m, 2H), $7.41-7.34(\mathrm{~m}, 2 \mathrm{H}), 7.21-7.15(\mathrm{~m}, 1 \mathrm{H})$, 5.98 (ddt, $J=17.2,10.4,5.6 \mathrm{~Hz}, 1 \mathrm{H}), 5.36$ (dq, $J=17.2,1.5 \mathrm{~Hz}, 1 \mathrm{H}), 5.27$ (ddd, $J=10.4$, 2.6, $1.3 \mathrm{~Hz}, 1 \mathrm{H}), 4.84-4.70(\mathrm{~m}, 2 \mathrm{H}) ;{ }^{13} \mathrm{C} \mathrm{NMR}\left(100 \mathrm{MHz}, \mathrm{CDCl}_{3}\right): \delta 164.9,132.3,129.1$, 126.0, 125.6, 124.2, 118.5, 65.6; HRMS (ESI TOF): Calculated for $\mathrm{C}_{11} \mathrm{H}_{10} \mathrm{~N}_{2} \mathrm{O}_{2}(\mathrm{M})^{+}$: 202.0742, Found: 202.0760 .

\section{2,2,2-trifluoroethyl 2-diazo-2-phenylacetate (1a'"'):}<smiles>N#CC(=O)OCC(F)(F)F</smiles>

Compound 1a'"' was synthesized following the general procedure (B). The product was obtained as orange solid (1.48 g, 76\% yield): $R_{f}=0.55$ petroleum ether/EtOAc (95:5); m.p.: 55-57 ${ }^{\circ} \mathrm{C}$; IR (neat) $\mathrm{cm}^{-1}: 3067,2985,2099,1719,1502,1413,1355,1288,1245,1170$, 1078, 972, 758, 692, 570; ${ }^{1} \mathrm{H}$ NMR (400 MHz, $\left.\mathrm{CDCl}_{3}\right): \delta(\mathrm{ppm}) 7.50-7.39(\mathrm{~m}, 4 \mathrm{H}), 7.26-$ 
$7.21(\mathrm{~m}, 1 \mathrm{H}), 4.66(\mathrm{q}, J=8.4 \mathrm{~Hz}, 2 \mathrm{H}) .{ }^{13} \mathrm{C} \mathrm{NMR}\left(100 \mathrm{MHz}, \mathrm{CDCl}_{3}\right): \delta(\mathrm{ppm})$ 163.32, $129.23,126.53,124.61,124.20,123.05$ (q, $J=277.5 \mathrm{~Hz}), 63.56,60.41$ (q, $J=36.9 \mathrm{~Hz}) .{ }^{19} \mathrm{~F}$ NMR (377 MHz, $\left.\mathrm{CDCl}_{3}\right): \delta$ (ppm) -73.94. HRMS (ESI TOF): Calculated for $\mathrm{C}_{10} \mathrm{H}_{8} \mathrm{~F}_{3} \mathrm{~N}_{2} \mathrm{O}_{2}$ $(\mathrm{M}+\mathrm{H})^{+}:$245.0528, Found: 245.0534 .

Prop-2-yn-1-yl 2-(4-bromophenyl)-2-diazoacetate (1b):<smiles>C#CCOC(=O)C(=N)c1ccc(Br)cc1</smiles>

Compound $\mathbf{1 b}$ was synthesized following the general procedure (B). The product was obtained as orange solid (2.261 g, 81\% yield): $R_{f}=0.45$ petroleum ether/EtOAc (95:5); m.p.: 57-59 ${ }^{\circ} \mathrm{C}$; IR (neat) $\mathrm{cm}^{-1}: 3294,2948,2085,1695,1488,1336,1236,1144,1030,999,948$, 813, 678, 632; ${ }^{1} \mathrm{H}$ NMR (400 MHz, $\left.\mathrm{CDCl}_{3}\right): \delta 7.54-7.47(\mathrm{~m}, 2 \mathrm{H}), 7.38-7.33(\mathrm{~m}, 2 \mathrm{H}), 4.87$ $(\mathrm{d}, J=2.3 \mathrm{~Hz}, 2 \mathrm{H}), 2.52(\mathrm{t}, J=2.3 \mathrm{~Hz}, 1 \mathrm{H}) ;{ }^{13} \mathrm{C} \mathrm{NMR}\left(100 \mathrm{MHz}, \mathrm{CDCl}_{3}\right): \delta 164.0,132.2$, 125.5, 124.4, 119.7, 77.6, 75.5, 52.5; HRMS (ESI TOF): Calculated for $\mathrm{C}_{11} \mathrm{H}_{8} \mathrm{BrN}_{2} \mathrm{O}_{2}(\mathrm{M}+$ $\mathrm{H})^{+}: 278.9769,280.9749$, Found:278.9722, 280.9739.

\section{Prop-2-yn-1-yl 2-(4-chlorophenyl)-2-diazoacetate (1c):}<smiles>C#CCOC(=O)C(=N)c1ccc(Cl)cc1</smiles>

Compound 1c was synthesized following the general procedure (B). The product was obtained as orange solid (1.736 g, 74\% yield). $R_{f}=0.35$ petroleum ether/EtOAc (95:5); m.p.: 61-63 ${ }^{\circ} \mathrm{C}$; IR (neat) $\mathrm{cm}^{-1}: 3295,2949,2086,1697,1492,1336,1276,1237,1146,1093,1005$, 820, 734, 680, 637; ${ }^{1} \mathrm{H}$ NMR (400 MHz, $\left.\mathrm{CDCl}_{3}\right): \delta 7.45-7.39$ (m, 2H), $7.38-7.33(\mathrm{~m}, 2 \mathrm{H})$, $4.87(\mathrm{~d}, J=2.5 \mathrm{~Hz}, 2 \mathrm{H}), 2.52(\mathrm{t}, J=2.5 \mathrm{~Hz}, 1 \mathrm{H}) ;{ }^{13} \mathrm{C} \mathrm{NMR}\left(100 \mathrm{MHz}, \mathrm{CDCl}_{3}\right): \delta 164.1$, 131.9, 129.3, 125.3, 123.8, 77.6, 75.4, 52.5; HRMS (ESI TOF): Calculated for $\mathrm{C}_{11} \mathrm{H}_{7} \mathrm{ClN}_{2} \mathrm{NaO}_{2}(\mathrm{M}+\mathrm{Na})^{+}:$257.0094, Found: 257.0097. 


\section{Prop-2-yn-1-yl 2-diazo-2-(4-fluorophenyl)acetate (1d):}<smiles>C#CCOC(=O)C(=N)c1ccc(F)cc1</smiles>

Compound 1d was synthesized following the general procedure (B). The product was obtained as yellow solid (1.833 g, 84\% yield). $R_{f}=0.3$ petroleum ether/EtOAc (95:5); m.p.: 62-64 ${ }^{\circ} \mathrm{C}$; IR (neat) $\mathrm{cm}^{-1}: 3298,2950,2086,1696,1508,1339,1286,1233,1145,1033,829$, 683, 642; ${ }^{1} \mathrm{H}$ NMR (400 MHz, $\left.\mathrm{CDCl}_{3}\right): \delta 7.51$ - $7.38(\mathrm{~m}, 2 \mathrm{H}), 7.17$ - $7.02(\mathrm{~m}, 2 \mathrm{H}), 4.87$ (d, $J$ $=2.5 \mathrm{~Hz}, 2 \mathrm{H}), 2.52(\mathrm{t}, J=2.5 \mathrm{~Hz}, 1 \mathrm{H}) ;{ }^{13} \mathrm{C} \mathrm{NMR}\left(100 \mathrm{MHz}, \mathrm{CDCl}_{3}\right): \delta 164.4,161.3(\mathrm{~d}, J=$ $246.7 \mathrm{~Hz}), 126.1$ (d, $J=8.0 \mathrm{~Hz}), 120.9$ (d, $J=3.2 \mathrm{~Hz}), 116.2$ (d, $J=22.0 \mathrm{~Hz}), 77.7,75.4$, 52.4; HRMS (ESI TOF): Calculated for $\mathrm{C}_{11} \mathrm{H}_{7} \mathrm{FN}_{2} \mathrm{NaO}_{2}(\mathrm{M}+\mathrm{Na})^{+}$: 241.0389, Found: 241.0384 .

\section{Prop-2-yn-1-yl 2-diazo-2-(3,4-dichlorophenyl)acetate (1e):}<smiles>C#CCOC(=O)C(=N)c1ccc(Cl)c(Cl)c1</smiles>

Compound 1e was synthesized following the general procedure (B). The product was obtained as orange solid (2.206 g, 82\% yield): $R_{f}=0.35$ petroleum ether/EtOAc (95:5); m.p.: $52-54{ }^{\circ} \mathrm{C}$; IR (neat) $\mathrm{cm}^{-1}: 3297,2950,2090,1698,1589,1475,1274,1149,1039,956,884$, 815, 736, 680, 640; ${ }^{1} \mathrm{H}$ NMR (400 MHz, $\left.\mathrm{CDCl}_{3}\right): \delta 7.65$ (d, $\left.J=2.3 \mathrm{~Hz}, 1 \mathrm{H}\right), 7.44(\mathrm{~d}, J=8.6$ $\mathrm{Hz}, 1 \mathrm{H}), 7.28(\mathrm{dd}, J=8.6,2.4 \mathrm{~Hz}, 1 \mathrm{H}), 4.87(\mathrm{~d}, J=2.6 \mathrm{~Hz}, 2 \mathrm{H}), 2.53(\mathrm{t}, J=2.4 \mathrm{~Hz}, 1 \mathrm{H}) ;{ }^{13} \mathrm{C}$ NMR (100 MHz, $\left.\mathrm{CDCl}_{3}\right): \delta$ 163.6, 133.5, 130.9, 129.8, 125.7, 125.4, 122.8, 77.4, 75.6, 52.6; HRMS (ESI TOF): Calculated for $\mathrm{C}_{11} \mathrm{H}_{6} \mathrm{Cl}_{2} \mathrm{~N}_{2} \mathrm{NaO}_{2}(\mathrm{M}+\mathrm{Na})^{+}:$290.9704, Found: 290.9701.

\section{Prop-2-yn-1-yl 2-diazo-2-(3,5-difluorophenyl)acetate (1f):}<smiles>C#CCOC(=O)C(=N)c1cc(F)cc(F)c1</smiles>

Compound $1 \mathbf{f}$ was synthesized following the general procedure (B). The product was obtained as orange solid (1.842 g, 78\% yield): $R_{f}=0.4$ petroleum ether/EtOAc (95:5); m.p.: 
36-38 ${ }^{\circ} \mathrm{C}$; IR (neat) $\mathrm{cm}^{-1}: 3301,3099,2953,2094,1704,1623,1589,1479,1445,1269,1124$, 1067, 987, 926, 839, 741, 669, 641; ${ }^{1} \mathrm{H}$ NMR (400 MHz, $\left.\mathrm{CDCl}_{3}\right): \delta 7.26-6.82(\mathrm{~m}, 2 \mathrm{H})$, 6.65-6.59 (m, 1H), $4.88(\mathrm{~d}, J=2.5 \mathrm{~Hz}, 2 \mathrm{H}), 2.54(\mathrm{t}, J=2.5 \mathrm{~Hz}, 1 \mathrm{H}) ;{ }^{13} \mathrm{C}$ NMR $(100 \mathrm{MHz}$, $\left.\mathrm{CDCl}_{3}\right): \delta 163.5(\mathrm{dd}, J=247.8,13.7 \mathrm{~Hz}), 163.3,129.2(\mathrm{t}, J=11.5 \mathrm{~Hz}), 106.7-106.2(\mathrm{~m})$, 101.2 (t, $J=25.5 \mathrm{~Hz}$ ), 77.3, 75.6, 52.6; HRMS (ESI TOF): Calculated for $\mathrm{C}_{11} \mathrm{H}_{6} \mathrm{~F}_{2} \mathrm{~N}_{2} \mathrm{NaO}_{2}$ $(\mathrm{M}+\mathrm{Na})^{+}: 259.0294$, Found: 259.0281 .

\section{Prop-2-yn-1-yl 2-diazo-2-(naphthalen-2-yl)acetate (1g):}<smiles>C#CCOC(=O)C(=N)c1ccc2ccccc2c1</smiles>

Compound $1 \mathrm{~g}$ was synthesized following the general procedure (B). The product was obtained as pale orange solid (1.927 g, 77\% yield). $R_{f}=0.35$ petroleum ether/EtOAc (95:5); m.p.: 82-84 ${ }^{\circ} \mathrm{C}$; IR (neat) $\mathrm{cm}^{-1}: 3291,3057,2947,2083,1698,1323,1248,1147,1121,1038$, 895, 852, 812, 736, 680, 639; ${ }^{1} \mathrm{H}$ NMR (400 MHz, $\left.\mathrm{CDCl}_{3}\right): \delta 8.01(\mathrm{~d}, J=1.7 \mathrm{~Hz}, 1 \mathrm{H}), 7.92-$ $7.69(\mathrm{~m}, 3 \mathrm{H}), 7.58-7.37(\mathrm{~m}, 3 \mathrm{H}), 4.91(\mathrm{~d}, J=2.5 \mathrm{~Hz}, 2 \mathrm{H}), 2.53(\mathrm{t}, J=2.5 \mathrm{~Hz}, 1 \mathrm{H}) ;{ }^{13} \mathrm{C}$ NMR (100 MHz, $\left.\mathrm{CDCl}_{3}\right): \delta 164.5,133.7,131.7,128.9,127.8,127.8,126.8,126.0,122.8$, 122.3, 121.9, 77.7, 75.4, 52.4; HRMS (ESI TOF): Calculated for $\mathrm{C}_{15} \mathrm{H}_{10} \mathrm{~N}_{2} \mathrm{NaO}_{2}(\mathrm{M}+\mathrm{Na})^{+}$: 273.0640, Found: 273.0640 .

\section{Prop-2-yn-1-yl 2-diazo-2-(p-tolyl)acetate (1h):}<smiles>C#CCOC(=O)C(=N)c1ccc(C)cc1</smiles>

Compound $1 \mathrm{~h}$ was synthesized following the general procedure (B). The product was obtained as orange solid (1.392 g, 65\% yield): $R_{f}=0.35$ petroleum ether/EtOAc (95:5); m.p.: $52-54{ }^{\circ} \mathrm{C}$; IR (neat) $\mathrm{cm}^{-1}: 3293,2947,2084,1699,1567,1514,1242,1154,1037,949,811$, 771, 735, 680, 640; ${ }^{1} \mathrm{H}$ NMR (400 MHz, $\left.\mathrm{CDCl}_{3}\right): \delta 7.36$ (d, $\left.J=8.0 \mathrm{~Hz}, 2 \mathrm{H}\right), 7.20(\mathrm{~d}, J=8.0$ $\mathrm{Hz}, 2 \mathrm{H}), 4.86(\mathrm{~d}, J=2.5 \mathrm{~Hz}, 2 \mathrm{H}), 2.51(\mathrm{t}, J=2.5 \mathrm{~Hz}, 1 \mathrm{H}), 2.34(\mathrm{~s}, 3 \mathrm{H}) ;{ }^{13} \mathrm{C} \mathrm{NMR}(100 \mathrm{MHz}$, $\left.\mathrm{CDCl}_{3}\right): \delta 164.7,136.1,129.9,124.3,121.8,77.8,75.2,52.3,21.1$; HRMS (ESI TOF): Calculated for $\mathrm{C}_{12} \mathrm{H}_{10} \mathrm{~N}_{2} \mathrm{NaO}_{2}(\mathrm{M}+\mathrm{Na})^{+}$: 237.0640, Found: 237.0635. 


\section{Prop-2-yn-1-yl 2-diazo-2-(4-methoxyphenyl)acetate (1i):}<smiles>C#CCOC(=O)C(C#N)c1ccc(OC)cc1</smiles>

Compound 1i was synthesized following the general procedure (B). The product was obtained as dark orange solid (1.934 g, 84\% yield): $R_{f}=0.3$ petroleum ether/EtOAc (95:5); m.p.: 68-69 ${ }^{\circ} \mathrm{C}$; IR (neat) $\mathrm{cm}^{-1}: 3289,2949,2837,2081,1694,1609,1573,1510,1240,1142$, 1022, 949, 825, 735, 680, 640; ${ }^{1} \mathrm{H}$ NMR (400 MHz, $\left.\mathrm{CDCl}_{3}\right): \delta 7.48-7.30(\mathrm{~m}, 2 \mathrm{H}), 7.09-$ $6.81(\mathrm{~m}, 2 \mathrm{H}), 4.86(\mathrm{~d}, J=2.4 \mathrm{~Hz}, 2 \mathrm{H}), 3.81(\mathrm{~s}, 3 \mathrm{H}), 2.51(\mathrm{t}, J=2.4 \mathrm{~Hz}, 1 \mathrm{H}) ;{ }^{13} \mathrm{C} \mathrm{NMR}(100$ $\left.\mathrm{MHz}, \mathrm{CDCl}_{3}\right): \delta 165.0,158.3,126.2,116.5,114.8,77.8,75.2,55.5,52.3$; HRMS (ESI TOF): Calculated for $\mathrm{C}_{12} \mathrm{H}_{10} \mathrm{~N}_{2} \mathrm{NaO}_{3}(\mathrm{M}+\mathrm{Na})^{+}$: 253.0589, Found: 253.0584.

\section{Prop-2-yn-1-yl 2-diazo-2-(3,5-dimethoxyphenyl)acetate (1j):}<smiles>C#CCOC(=O)C(=N)c1cc(OC)cc(OC)c1</smiles>

Compound $\mathbf{1} \mathbf{j}$ was synthesized following the general procedure (B). The product was obtained as yellow solid (1.822 g, 70\% yield): $R_{f}=0.25$ petroleum ether/EtOAc (95:5); m.p.: 91-93 ${ }^{\circ} \mathrm{C}$; IR (neat) $\mathrm{cm}^{-1}: 3254,2946,2839,2075,1703,1594,1454,1383,1324,1272,1154$, 1035, 954, 926, 830, 736, 672; ${ }^{1} \mathrm{H}$ NMR (400 MHz, $\left.\mathrm{CDCl}_{3}\right): \delta 6.67$ (d, $\left.J=2.2 \mathrm{~Hz}, 2 \mathrm{H}\right), 6.30$ $(\mathrm{t}, J=2.2 \mathrm{~Hz}, 1 \mathrm{H}), 4.86(\mathrm{~d}, J=2.5 \mathrm{~Hz}, 2 \mathrm{H}), 3.80(\mathrm{~s}, 6 \mathrm{H}), 2.52(\mathrm{t}, J=2.5 \mathrm{~Hz}, 1 \mathrm{H}) ;{ }^{13} \mathrm{C} \mathrm{NMR}$ (100 MHz, $\left.\mathrm{CDCl}_{3}\right): \delta 164.2,161.3,127.3,102.1,98.4,77.7,75.3,55.5,52.3$; HRMS (ESI TOF): Calculated for $\mathrm{C}_{13} \mathrm{H}_{12} \mathrm{~N}_{2} \mathrm{NaO}_{4}(\mathrm{M}+\mathrm{Na})^{+}:$283.0695, Found: 283.0695 .

\section{General Procedure C1 for optimization of reaction conditions}

A mixture of $5 \mathrm{~mol} \%$ of catalyst and $1.5 \mathrm{~mL}$ of benzene $2 \mathrm{a}$ was stirred at room temperature in a dried $25 \mathrm{~mL}$ two-necked RB flask under an inert atmosphere for 5 minutes. Then $\alpha$-aryl- $\alpha$-diazoacetate $1(0.5 \mathrm{mmol})$ in $1 \mathrm{~mL}$ of benzene was added to above reaction mixture dropwise over 5 minutes, and then the reaction mixture was stirred at room temperature or refluxed at $80{ }^{\circ} \mathrm{C}$ over $4-12 \mathrm{~h}$. The progress of the reaction was monitored by TLC. After completion of the reaction, the solvent was removed under reduced pressure. The 
crude product obtained was purified by column chromatography over silica gel to furnish the product 3 .

\section{General procedure $\mathrm{C} 2$ for screening of solvents}

In a dried $25 \mathrm{~mL} \mathrm{RB}$ flask, Sc(OTf) 3 (2.5-5 mol\%), propargyl $\alpha$-phenyl- $\alpha$-diazoacetate $1 \mathrm{a}(0.1 \mathrm{~g}, 0.5 \mathrm{mmol})$ and benzene $2 \mathrm{a}(0.45 \mathrm{~mL}, 5 \mathrm{mmol})$ were added in $2.5 \mathrm{~mL}$ of solvent at room temperature under inert atmosphere. Then the reaction mixture was refluxed at $72-80$ ${ }^{\circ} \mathrm{C}$ over 4-12 h. The progress of the reaction was monitored by TLC. After completion of the reaction, the solvent was removed under reduced pressure. The crude product obtained was purified by column chromatography over silica gel to furnish the desired product 3aa (see Appendix-I).

Appendix-I Screening of solvents ${ }^{\mathrm{a}-\mathrm{c}}$<smiles>C#CCOC(=O)C(=N)c1ccccc1</smiles>

$1 a$<smiles>c1ccccc1</smiles>

$2 a$
$\mathrm{Sc}(\mathrm{OTf})_{3}(2.5-5 \mathrm{~mol} \%)$

Solvent, temp, 4-12 h

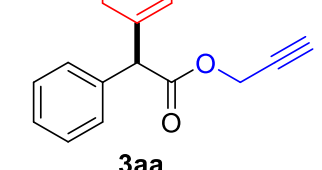

\begin{tabular}{clcccc}
\hline Entry & Solvent & $\begin{array}{c}\text { Sc(OTf) } \\
(\mathbf{m o l} \%)\end{array}$ & $\begin{array}{c}\text { Temp } \\
\left({ }^{\circ} \mathbf{C}\right)\end{array}$ & $\begin{array}{c}\text { Time } \\
(\mathbf{h})\end{array}$ & $\begin{array}{c}\text { Yield }^{\mathbf{b}, \mathbf{c}} \\
(\mathbf{\%})\end{array}$ \\
\hline 1 & EtOAc & 5 & 78 & 8 & 39 \\
2 & Nitromethane & 5 & 80 & 4 & 45 \\
3 & DCM & 5 & 72 & 12 & 48 \\
$\mathbf{4}$ & DCE & $\mathbf{5}$ & $\mathbf{8 0}$ & $\mathbf{4}$ & $\mathbf{7 2}$ \\
5 & DMF & 5 & 80 & 12 & NR \\
6 & Acetonitrile & 5 & 80 & 12 & NR \\
7 & DCE & 2.5 & 80 & 8 & 40 \\
8 & THF & - & - & - & - \\
\hline
\end{tabular}

${ }^{a}$ Reaction conditions: propargyl $\alpha$-phenyl- $\alpha$-diazoacetate 1a $\left(0.5 \mathrm{mmol}, 1\right.$ equiv.), $\mathrm{Sc}(\mathrm{OTf})_{3}(5 \mathrm{~mol} \%)$, and benzene $2 \mathbf{a}$ ( $5 \mathrm{mmol}, 10$ equiv.) was added in solvent $(2.5 \mathrm{~mL})$ under inert atmosphere and reaction mixture was refluxed at $72-80{ }^{\circ} \mathrm{C}$; the reaction was monitored by TLC. ${ }^{\mathrm{b}}$ Isolated yield after purification by column chromatography. ${ }^{\mathrm{c}} \mathrm{NR}$ - no reaction. 


\section{General procedure $\mathrm{C} 3$ for screening of co-catalyst}

In a dried $25 \mathrm{~mL} \mathrm{RB}$ flask, $\mathrm{Sc}(\mathrm{OTf})_{3}(2.5-5 \mathrm{~mol} \%)$, co-catalyst (0-5 mol\%), propargyl $\alpha$-phenyl- $\alpha$-diazoacetate $1 \mathbf{a}(0.1 \mathrm{~g}, 0.5 \mathrm{mmol})$ and benzene $\mathbf{2 a}(0.45 \mathrm{~mL}, 5 \mathrm{mmol})$ was added in $2.5 \mathrm{~mL}$ of $\mathrm{DCE}$ at room temperature under inert atmosphere. Then the reaction mixture was refluxed at $80{ }^{\circ} \mathrm{C}$ over 2-12 h. The progress of the reaction was monitored by TLC. After completion of the reaction, the solvent was removed under reduced pressure. The crude product obtained was purified by column chromatography over silica gel to furnish the desired product 3aa (see Appendix-II).

Appendix-II Screening of co-catalyst ${ }^{\mathrm{a}-\mathrm{c}}$

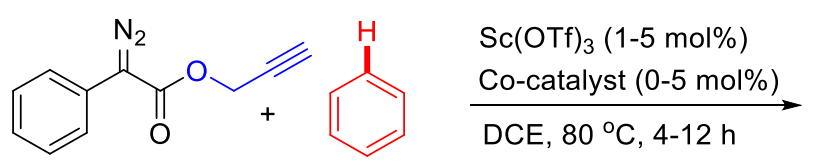

$1 \mathbf{a}$

$2 a$

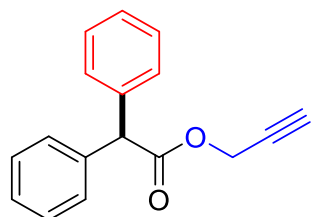

3aa

\begin{tabular}{cccccc}
\hline Entry & Solvent & $\begin{array}{c}\text { Sc(OTf) } \\
(\mathbf{m o l} \%)\end{array}$ & $\begin{array}{c}\text { Co-catalyst } \\
(\mathbf{m o l} \%)\end{array}$ & $\begin{array}{c}\text { Time } \\
\text { (h) }\end{array}$ & $\begin{array}{c}\text { Yield (\%) } \\
\text { 3aa }\end{array}$ \\
\hline 1 & DCE & 2 & $\operatorname{AgSbF}_{6}(5)$ & 4 & 20 \\
2 & DCE & 5 & $\operatorname{AgSbF}_{6}(5)$ & 2 & 40 \\
3 & DCE & 1 & $\operatorname{AgSbF}_{6}(5)$ & 12 & 10 \\
4 & DCE & 5 & $\operatorname{AgOTf}^{\mathbf{c}}(5)$ & 12 & DC \\
5 & DCE & 2 & $\operatorname{AgOTf}(5)$ & 12 & DC \\
$\mathbf{6}$ & DCE & $\mathbf{5}$ & - & $\mathbf{4}$ & $\mathbf{7 2}$ \\
\hline
\end{tabular}

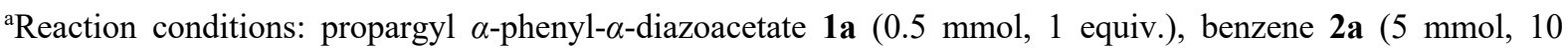
equiv.), Catalyst (1-5 mol\%) and co-catalyst (5 mol\%) was added in DCE (2.5 mL) under inert atmosphere and reaction mixture was refluxed at $80{ }^{\circ} \mathrm{C}$; the reaction was monitored by TLC. ${ }^{\mathrm{b}}$ Isolated yield after purification by column chromatography. ${ }^{\mathrm{c} D C}$ - decomposed.

\section{General procedure D for synthesis of $\alpha$-diarylacetates}

In a dried $25 \mathrm{~mL} \mathrm{RB}$ flask, $\mathrm{Sc}(\mathrm{OTf})_{3}(12.3 \mathrm{mg}, 5 \mathrm{~mol} \%)$, propargyl $\alpha$-phenyl- $\alpha$ diazoacetate $1 \mathrm{a}(0.1 \mathrm{~g}, 0.5 \mathrm{mmol})$ and benzene $2 \mathrm{a}(0.45 \mathrm{~mL}, 5 \mathrm{mmol})$ was added in $2.5 \mathrm{~mL}$ of DCE at room temperature under inert atmosphere. Then the reaction mixture was refluxed at $80{ }^{\circ} \mathrm{C}$ over $4 \mathrm{~h}$. The progress of the reaction was monitored by TLC. After completion of the reaction, the solvent was removed under reduced pressure. The crude product obtained 
was purified by column chromatography over silica gel to furnish the desired product 3aa (0.09 g, 72\% yield).

\section{Prop-2-yn-1-yl 2,2-diphenylacetate (3aa):}<smiles>C#CCOC(=O)C(c1ccccc1)c1ccccc1</smiles>

Compound 3aa was synthesized following the general procedure (D). The product was obtained as white solid ( $0.09 \mathrm{~g}, 72 \%$ yield): $R_{f}=0.5$ petroleum ether/EtOAc (95:5); m.p.: 46-48 ${ }^{\circ} \mathrm{C}$; IR (neat) $\mathrm{cm}^{-1}: 3289,3031,2941,2126,1739,1598,1494,1448,1370,1220,1181$, 1080, 997, 739, 694, 638, 564; ${ }^{1} \mathrm{H}$ NMR (400 MHz, $\left.\mathrm{CDCl}_{3}\right): \delta 7.49-7.12$ (m, 10H), 5.07 (s, $1 \mathrm{H}), 4.74(\mathrm{~d}, J=2.5 \mathrm{~Hz}, 2 \mathrm{H}), 2.46(\mathrm{t}, J=2.5 \mathrm{~Hz}, 1 \mathrm{H}) ;{ }^{13} \mathrm{C} \mathrm{NMR}\left(100 \mathrm{MHz}, \mathrm{CDCl}_{3}\right): \delta 171.8$, 138.3, 128.8, 128.7, 127.5, 77.4, 75.3, 56.8, 52.7; HRMS (ESI TOF): Calculated for $\mathrm{C}_{17} \mathrm{H}_{14} \mathrm{NaO}_{2}(\mathrm{M}+\mathrm{Na})^{+}: 273.0891$, Found: 273.0891 .

\section{Ethyl 2,2-diphenylacetate (3a'a):}<smiles>CCOC(=O)C(c1ccccc1)c1ccccc1</smiles>

Compound 3a'a was synthesized following the general procedure (D). The product was obtained as colourless liquid $\left(0.037 \mathrm{~g}, 31 \%\right.$ yield): $R_{f}=0.45$ petroleum ether/EtOAc (95:5); IR (neat) $\mathrm{cm}^{-1}:$ 3029, 2984, 1730, 1598, 1493, 1452, 1148, 1093, 1025, 961, 697, 633, 561; ${ }^{1} \mathrm{H}$ NMR (400 MHz, $\left.\mathrm{CDCl}_{3}\right): \delta 7.35$ - $7.29(\mathrm{~m}, 8 \mathrm{H}), 7.28-7.22(\mathrm{~m}, 2 \mathrm{H}), 5.01(\mathrm{~s}, 1 \mathrm{H})$, $4.20(\mathrm{q}, J=7.1 \mathrm{~Hz}, 2 \mathrm{H}), 1.25(\mathrm{t}, J=7.1 \mathrm{~Hz}, 3 \mathrm{H}) ;{ }^{13} \mathrm{C} \mathrm{NMR}\left(100 \mathrm{MHz}, \mathrm{CDCl}_{3}\right): \delta$ 172.6, 138.9, 128.7, 128.7, 127.3, 61.3, 57.3, 14.3; HRMS (ESI TOF): Calculated for $\mathrm{C}_{16} \mathrm{H}_{16} \mathrm{NaO}_{2}$ $(\mathrm{M}+\mathrm{Na})^{+}: 263.1048$, Found: 263.1042. 


\section{Allyl 2,2-diphenylacetate (3a"a):}<smiles>C=CCOC(=O)C(c1ccccc1)c1ccccc1</smiles>

Compound 3a"a was synthesized following the general procedure (D). The product was obtained as colourless liquid $\left(0.042 \mathrm{~g}, 33 \%\right.$ yield): $R_{f}=0.6$ petroleum ether/EtOAc (95:5); IR(neat) $\mathrm{cm}^{-1}: 3029,2942,2109,1734,1648,1494,1450,1184,1145,1083,989,931$, 740, 700, 624, 559; ${ }^{1} \mathrm{H}$ NMR (400 MHz, $\left.\mathrm{CDCl}_{3}\right): \delta 7.38-7.17$ (m, 10H), 5.89 (ddt, $J=15.4$, 10.4, $5.7 \mathrm{~Hz}, 1 \mathrm{H}), 5.22$ (ddq, $J=15.4,10.4,1.4 \mathrm{~Hz}, 1 \mathrm{H}), 5.05$ (s, 1H), 4.65 (dt, $J=5.7,1.4$ $\mathrm{Hz}, 1 \mathrm{H}) ;{ }^{13} \mathrm{C} \mathrm{NMR}\left(100 \mathrm{MHz}, \mathrm{CDCl}_{3}\right): \delta 172.3,138.8,132.0,128.8,128.7,127.4,118.6$, 65.9, 57.2; HRMS (ESI TOF): Calculated for $\mathrm{C}_{17} \mathrm{H}_{16} \mathrm{NaO}_{2}(\mathrm{M}+\mathrm{Na})^{+}$: 275.1048, Found: 275.1044 .

\section{2,2,2-trifluoroethyl 2,2-diphenylacetate (3a'"'a):}<smiles>O=C(OCC(F)(F)F)C(c1ccccc1)c1ccccc1</smiles>

Compound 3a'"'a was synthesized following the general procedure (D). The product was obtained as colourless liquid $(0.063 \mathrm{~g}, 52 \%$ yield $): R_{f}=0.6$ petroleum ether/EtOAc (95:5); IR(neat) $\mathrm{cm}^{-1}: 3032,2945,1758,1497,1451,1408,1282,1170,1137,1047,976,845$, 741, 702, 646, 561. ${ }^{1} \mathrm{H}$ NMR (400 MHz, $\left.\mathrm{CDCl}_{3}\right): \delta(\mathrm{ppm}) 7.36-7.24(\mathrm{~m}, 10 \mathrm{H}), 5.12(\mathrm{~s}, 1 \mathrm{H})$, $4.51(\mathrm{q}, J=8.4 \mathrm{~Hz}, 2 \mathrm{H}) .{ }^{13} \mathrm{C} \mathrm{NMR}\left(101 \mathrm{MHz}, \mathrm{CDCl}_{3}\right): \delta(\mathrm{ppm}) 171.15,137.78,128.88$, 128.64, 127.75, 122.98 (q, $J=277.4 \mathrm{~Hz}), 60.82$ (q, $J=36.7 \mathrm{~Hz}), 56.61 .{ }^{19} \mathrm{~F} \mathrm{NMR}(377 \mathrm{MHz}$, $\left.\mathrm{CDCl}_{3}\right): \delta(\mathrm{ppm})$-73.57. HRMS (ESI TOF): Calculated for $\mathrm{C}_{16} \mathrm{H}_{14} \mathrm{~F}_{3} \mathrm{O}_{2}(\mathrm{M}+\mathrm{H})^{+}:$295.0946, Found: 295.0941. 


\section{Prop-2-yn-1-yl 2-(4-bromophenyl)-2-phenylacetate (3ba):}<smiles>C#CCOC(=O)C(c1ccccc1)c1ccc(Br)cc1</smiles>

Compound 3 ba was synthesized following the general procedure (D). The product was obtained as white solid ( $0.122 \mathrm{~g}, 74 \%$ yield): $R_{f}=0.45$ petroleum ether/EtOAc (95:5); m.p.: 104-106 ${ }^{\circ} \mathrm{C}$; IR (neat) $\mathrm{cm}^{-1}:$ 3292, 3032, 2940, 2126, 1739, 1597, 1488, 1271, 1368, 1139, 1002, 810, 746, 694, 639, 579; ${ }^{1} \mathrm{H}$ NMR (400 MHz, $\left.\mathrm{CDCl}_{3}\right): \delta 7.48-7.43(\mathrm{~m}, 2 \mathrm{H})$, $7.36-7.25(\mathrm{~m}, 5 \mathrm{H}), 7.22-7.17(\mathrm{~m}, 2 \mathrm{H}), 5.02(\mathrm{~s}, 1 \mathrm{H}), 4.85-4.62(\mathrm{~m}, 2 \mathrm{H}), 2.48(\mathrm{t}, J=2.5$ $\mathrm{Hz}, 1 \mathrm{H}) ;{ }^{13} \mathrm{C} \mathrm{NMR}\left(100 \mathrm{MHz}, \mathrm{CDCl}_{3}\right): \delta 171.4,137.8,137.3,131.9,130.5,128.9,128.6$, 127.8, 121.7, 77.3, 75.5, 56.2, 52.9; HRMS (ESI TOF): Calculated for $\mathrm{C}_{17} \mathrm{H}_{14}{ }^{81} \mathrm{BrO}_{2}(\mathrm{M}+$ $\mathrm{H})^{+}:$331.0157, Found: 331.0144 .

\section{Prop-2-yn-1-yl 2-(4-chlorophenyl)-2-phenylacetate (3ca):}<smiles>C#CCOC(=O)C(c1ccccc1)c1ccc(Cl)cc1</smiles>

Compound 3ca was synthesized following the general procedure (D). The product was obtained as white solid ( $0.114 \mathrm{~g}, 80 \%$ yield): $R_{f}=0.4$ petroleum ether/EtOAc (95:5); m.p.: 83-85 ${ }^{\circ} \mathrm{C}$; IR (neat) $\mathrm{cm}^{-1}: 3293,3033,2127,1739,1598,1490,1272,1222,1138$, 1001, 813, 751, 691, 636, 550; ${ }^{1} \mathrm{H}$ NMR (400 MHz, $\left.\mathrm{CDCl}_{3}\right): \delta 7.35$ - 7.24 (m, 9H), 5.03 (s, 1H), $4.79-4.69$ (m, 2H), 2.47 (t, $J=2.5 \mathrm{~Hz}, 1 \mathrm{H}) ;{ }^{13} \mathrm{C}$ NMR (100 MHz, DMSO-d 6 ): $\delta$ 171.0, 138.4, 137.7, 132.0, 130.4, 128.7, 128.5, 128.3, 127.3, 78.1 (for 2-C), 54.5, 52.6; HRMS (ESI TOF): Calculated for $\mathrm{C}_{17} \mathrm{H}_{13} \mathrm{ClNaO}_{2}(\mathrm{M}+\mathrm{Na})^{+}$: 307.0502, Found: 307.0505 . 


\section{Prop-2-yn-1-yl 2-(4-fluorophenyl)-2-phenylacetate (3da):}<smiles>C#CCOC(=O)C(c1ccccc1)c1ccc(F)cc1</smiles>

Compound 3da was synthesized following the general procedure (D). The product was obtained as white solid (0.094 g, 70\% yield): $R_{f}=0.4$ petroleum ether/EtOAc (95:5); m.p.: $65-67{ }^{\circ} \mathrm{C}$; IR (neat) $\mathrm{cm}^{-1}: 3293,3064,2130,1739,1604,1506,1448,1370,1276$, 1182, 1137, 996, 809, 732, 692, 636; ${ }^{1} \mathrm{H}$ NMR (400 MHz, $\left.\mathrm{CDCl}_{3}\right): \delta 7.38-7.25(\mathrm{~m}, 7 \mathrm{H})$, $7.09-6.92(\mathrm{~m}, 2 \mathrm{H}), 5.05(\mathrm{~s}, 1 \mathrm{H}), 4.78-4.67(\mathrm{~m}, 2 \mathrm{H}), 2.47(\mathrm{t}, J=2.5 \mathrm{~Hz}, 1 \mathrm{H}) ;{ }^{13} \mathrm{C} \mathrm{NMR}$ $\left(100 \mathrm{MHz}, \mathrm{CDCl}_{3}\right): \delta 171.7,162.2(\mathrm{~d}, J=246.3 \mathrm{~Hz}), 138.2,134.1(\mathrm{~d}, J=3.3 \mathrm{~Hz}), 130.4(\mathrm{~d}, J$ $=8.1 \mathrm{~Hz}), 128.9,128.6,127.7,115.6(\mathrm{~d}, J=21.5 \mathrm{~Hz}), 77.4,75.4,56.0,52.8$; HRMS (ESI TOF): Calculated for $\mathrm{C}_{17} \mathrm{H}_{14} \mathrm{FO}_{2}(\mathrm{M}+\mathrm{H})^{+}:$269.0978, Found: 269.0980.

\section{Prop-2-yn-1-yl 2-(3,4-dichlorophenyl)-2-phenylacetate (3ea):}<smiles>C#CCOC(=O)C(c1ccccc1)c1ccc(Cl)c(Cl)c1</smiles>

Compound 3ea was synthesized following the general procedure (D). The product was obtained as colourless liquid $(0.136 \mathrm{~g}, 85 \%$ yield $): R_{f}=0.35$ petroleum ether/EtOAc (95:5); IR (neat) $\mathrm{cm}^{-1}: 3295,3071,2948,2124,1743,1596,1470,1269,1145,1028,907$, 772, 702; ${ }^{1} \mathrm{H}$ NMR (400 MHz, $\left.\mathrm{CDCl}_{3}\right): \delta 7.42(\mathrm{~d}, J=2.2 \mathrm{~Hz}, 1 \mathrm{H}), 7.41-7.26(\mathrm{~m}, 6 \mathrm{H}), 7.16$ $(\mathrm{dd}, J=8.3,2.2 \mathrm{~Hz}, 1 \mathrm{H}), 5.00(\mathrm{~s}, 1 \mathrm{H}), 4.80-4.69(\mathrm{~m}, 2 \mathrm{H}), 2.49(\mathrm{t}, J=2.5 \mathrm{~Hz}, 1 \mathrm{H}) ;{ }^{13} \mathrm{C}$ NMR (100 MHz, $\left.\mathrm{CDCl}_{3}\right)$ : $\delta 171.0,138.4,137.2,132.8,131.8,130.7,130.6,129.1,128.5$, 128.2, 128.0, 75.6(for 2-C), 55.8, 53.0; HRMS (ESI TOF): Calculated for $\mathrm{C}_{17} \mathrm{H}_{12} \mathrm{Cl}_{2} \mathrm{NaO}_{2}$ (M $+\mathrm{Na})^{+}:$341.0112, Found: 341.0099 . 


\section{Prop-2-yn-1-yl 2-(3,5-difluorophenyl)-2-phenylacetate (3fa):}<smiles>C#CCOC(=O)C(c1ccccc1)c1cc(F)cc(F)c1</smiles>

Compound 3fa was synthesized following the general procedure (D). The product was obtained as colourless liquid ( $0.139 \mathrm{~g}, 97 \%$ yield): $R_{f}=0.45$ petroleum ether/EtOAc (95:5); IR (neat) $\mathrm{cm}^{-1}: 3297,3091,2130,1742,1623,1597,1495,1450,1302,1219,1146,1119$, 1026, 987, 848, 770, 697; ${ }^{1} \mathrm{H}$ NMR (400 MHz, $\left.\mathrm{CDCl}_{3}\right): \delta 7.49-7.27$ (m, 5H), 6.98 - 6.79 $(\mathrm{m}, 2 \mathrm{H}), 6.71(\mathrm{tt}, J=8.8,2.3 \mathrm{~Hz}, 1 \mathrm{H}), 5.01(\mathrm{~s}, 1 \mathrm{H}), 4.92-4.48(\mathrm{~m}, 2 \mathrm{H}), 2.49(\mathrm{t}, J=2.5 \mathrm{~Hz}$, $1 \mathrm{H}) ;{ }^{13} \mathrm{C} \mathrm{NMR}\left(100 \mathrm{MHz}, \mathrm{CDCl}_{3}\right): \delta 170.8,163.1(\mathrm{dd}, J=248.8,12.8 \mathrm{~Hz}), 142.0(\mathrm{t}, J=9.2$ Hz), 137.0, 129.1, 128.6, 128.1, $112.11-111.55$ (m), 103.1 (t, $J=25.3 \mathrm{~Hz}), 77.4$, 75.6, 56.2 $\left(\mathrm{t}, J=1.9 \mathrm{~Hz}\right.$ ), 53.0; HRMS (ESI TOF): Calculated for $\mathrm{C}_{17} \mathrm{H}_{13} \mathrm{~F}_{2} \mathrm{O}_{2}(\mathrm{M}+\mathrm{H})^{+}:$287.0884, Found: 287.0888.

\section{Prop-2-yn-1-yl 2-phenyl-2-(p-tolyl)acetate (3ha):}<smiles>C#CCOC(=O)C(c1ccccc1)c1ccc(C)cc1</smiles>

Compound 3ha was synthesized following the general procedure (D). The product was obtained as white solid ( $0.02 \mathrm{~g}, 15 \%$ yield): $R_{f}=0.5$ petroleum ether/EtOAc (95:5); m.p.: $88-90{ }^{\circ} \mathrm{C}$; IR (neat) $\mathrm{cm}^{-1}: 3290,3028,2927,2128,1742,1509,1448,1370,1221,1182,1141$, 999, 731, 695; ${ }^{1} \mathrm{H}$ NMR (400 MHz, $\left.\mathrm{CDCl}_{3}\right): \delta 7.34-7.24(\mathrm{~m}, 5 \mathrm{H}), 7.20$ (d, J=8.0 Hz, 2H), $7.13(\mathrm{~d}, J=8.0 \mathrm{~Hz}, 2 \mathrm{H}), 5.03(\mathrm{~s}, 1 \mathrm{H}), 4.73(\mathrm{~d}, J=2.5 \mathrm{~Hz}, 2 \mathrm{H}), 2.45$ (t, $J=2.5 \mathrm{~Hz}, 1 \mathrm{H}), 2.32$ (s, 3H); ${ }^{13} \mathrm{C}$ NMR (100 MHz, $\left.\mathrm{CDCl}_{3}\right): \delta 172.0,138.5,137.2,135.4,129.5,128.7,128.66$, 128.6, 127.4, 77.5, 75.3, 56.4, 52.7, 21.2; HRMS (ESI TOF): Calculated for $\mathrm{C}_{18} \mathrm{H}_{16} \mathrm{NaO}_{2}(\mathrm{M}$ $+\mathrm{Na})^{+}: 287.1048$, Found: 287.1040 . 


\section{Prop-2-yn-1-yl 2-(3,5-dimethoxyphenyl)-2-phenylacetate (3ja):}<smiles>C#CCOC(=O)C(c1ccccc1)c1cc(OC)cc(OC)c1</smiles>

Compound 3ja was synthesized following the general procedure (D). The product was obtained as colourless liquid ( $0.026 \mathrm{~g}, 17 \%$ yield): $R_{f}=0.5$ petroleum ether/EtOAc (80:20); IR (neat) $\mathrm{cm}^{-1}: 3284,2936,2841,2128,1740,1595,1460,1298,1201,1143,1062,999,938$, 834, 761; ${ }^{1} \mathrm{H}$ NMR (400 MHz, $\left.\mathrm{CDCl}_{3}\right): \delta 7.36$ - $7.22(\mathrm{~m}, 5 \mathrm{H}), 6.48(\mathrm{~d}, J=2.2 \mathrm{~Hz}, 2 \mathrm{H}), 6.37$ $(\mathrm{t}, J=2.2 \mathrm{~Hz}, 1 \mathrm{H}), 4.99(\mathrm{~s}, 1 \mathrm{H}), 4.74(\mathrm{~d}, J=2.5 \mathrm{~Hz}, 2 \mathrm{H}), 3.75(\mathrm{~s}, 6 \mathrm{H}), 2.46(\mathrm{t}, J=2.5 \mathrm{~Hz}$, $1 \mathrm{H}) ;{ }^{13} \mathrm{C} \mathrm{NMR}\left(100 \mathrm{MHz}, \mathrm{CDCl}_{3}\right): \delta 171.6,161.0,140.3,138.0,128.7,128.7,127.6,107.0$, 99.4, 77.5, 75.3, 56.9, 55.4, 52.7; HRMS (ESI TOF): Calculated for $\mathrm{C}_{19} \mathrm{H}_{19} \mathrm{O}_{4}(\mathrm{M}+\mathrm{H})^{+}$: 311.1283, Found: 311.1287.

\section{Prop-2-yn-1-yl 2-(4-fluorophenyl)-2-phenylacetate (3ab):}<smiles>C#CCOC(=O)C(c1ccccc1)c1ccc(F)cc1</smiles>

Compound 3ab was synthesized following the general procedure (D). The product was obtained as a major product para-isomer $(p: o=91: 9)$, $p: o$ ratio determined by ${ }^{1} \mathrm{H}$ NMR. The product was obtained as viscous colourless liquid $(0.054 \mathrm{~g}, 40 \%$ yield $): R_{f}=0.4$ petroleum ether/EtOAc (95:5); IR (neat) $\mathrm{cm}^{-1}:$ 3294, 3071, 2945, 2125, 1742, 1604, 1506, 1447, 1370, 1279, 1227, 1144, 1001, 816, 733, 695, 641, 560; ${ }^{1} \mathrm{H}$ NMR (400 MHz, $\left.\mathrm{CDCl}_{3}\right): \delta$ $7.51-7.18(\mathrm{~m}, 7 \mathrm{H}), 7.13-6.85(\mathrm{~m}, 2 \mathrm{H}), 5.05(\mathrm{~s}, 1 \mathrm{H}), 4.73(\mathrm{dd}, J=2.4,1.0 \mathrm{~Hz}, 2 \mathrm{H}), 2.46(\mathrm{t}$, $J=2.4 \mathrm{~Hz}, 1 \mathrm{H}) ;{ }^{13} \mathrm{C} \mathrm{NMR}\left(100 \mathrm{MHz}, \mathrm{CDCl}_{3}\right): \delta 171.7,162.2(\mathrm{~d}, J=246.3 \mathrm{~Hz}), 138.1,134.1$ (d, $J=2.9 \mathrm{~Hz}), 130.4$ (d, $J=8.0 \mathrm{~Hz}), 128.9,128.5,127.7,115.6$ (d, $J=21.3 \mathrm{~Hz}), 77.3,75.4$, 55.9, 52.8; HRMS (ESI TOF): Calculated for $\mathrm{C}_{17} \mathrm{H}_{14} \mathrm{FO}_{2}(\mathrm{M}+\mathrm{H})^{+}$: 269.0978, Found: 269.0988 . 


\section{Prop-2-yn-1-yl 2-(4-bromophenyl)-2-(4-fluorophenyl)acetate (3bb):}<smiles>C#CCOC(=O)C(c1ccc(F)cc1)c1ccc(Br)cc1</smiles>

Compound $\mathbf{3 b b}$ was synthesized following the general procedure (D). The product was obtained as a major product para-isomer $(p: o=91: 9)$, $p$ :o ratio determined by ${ }^{1} \mathrm{H}$ NMR. The product was obtained as semisolid $(0.090 \mathrm{~g}, 52 \%$ yield $): R_{f}=0.45$ petroleum ether/EtOAc (95:5); IR (neat) $\mathrm{cm}^{-1}:$ 3295, 3060, 2130, 1741, 1603, 1504, 1438, 1369, 1273, 1227, 1144, 950, 822, 760, 683, 641; ${ }^{1} \mathrm{H}$ NMR (400 MHz, $\left.\mathrm{CDCl}_{3}\right): \delta 7.50-7.41(\mathrm{~m}, 2 \mathrm{H})$, $7.30-7.22(\mathrm{~m}, 2 \mathrm{H}), 7.21-7.13(\mathrm{~m}, 2 \mathrm{H}), 7.07-6.94(\mathrm{~m}, 2 \mathrm{H}), 4.99(\mathrm{~s}, 1 \mathrm{H}), 4.74(\mathrm{dd}, J=3.8$, $2.5 \mathrm{~Hz}, 2 \mathrm{H}), 2.48(\mathrm{t}, J=2.5 \mathrm{~Hz}, 1 \mathrm{H}) ;{ }^{13} \mathrm{C} \mathrm{NMR}\left(100 \mathrm{MHz}, \mathrm{CDCl}_{3}\right): \delta 171.3,162.3(\mathrm{~d}, J=$ $246.9 \mathrm{~Hz}), 137.2,133.6(\mathrm{~d}, J=3.3 \mathrm{~Hz}), 132.0,130.3,130.2,121.8,115.8$ (d, $J=21.6 \mathrm{~Hz})$, 77.2, 75.5, 55.4, 53.0; HRMS (ESI TOF): Calculated for $\mathrm{C}_{17} \mathrm{H}_{13} \mathrm{BrFO}_{2}(\mathrm{M}+\mathrm{H})^{+}$: 347.0083 , Found: 347.0092 .

\section{Prop-2-yn-1-yl 2-(4-chlorophenyl)-2-(4-fluorophenyl)acetate (3cb):}<smiles>C#CCOC(=O)C(c1ccc(F)cc1)c1ccc(Cl)cc1</smiles>

Compound 3cb was synthesized following the general procedure (B). The product was obtained as a major product para-isomer $(p: o=91: 9)$, $p$ :o ratio determined by ${ }^{1} \mathrm{H}$ NMR. The product was obtained as colourless liquid $(0.082 \mathrm{~g}, 54 \%$ yield $) . R_{f}=0.45$ petroleum ether/EtOAc (95:5); IR (neat) $\mathrm{cm}^{-1}: 3297,2947,2130,1741,1602,1439,1270,1227,1143$, 1093, 1005, 823, 762, 683, 641; ${ }^{1} \mathrm{H}$ NMR (400 MHz, $\left.\mathrm{CDCl}_{3}\right): \delta 7.39-7.16(\mathrm{~m}, 6 \mathrm{H}), 7.15-$ $6.90(\mathrm{~m}, 2 \mathrm{H}), 5.01(\mathrm{~s}, 1 \mathrm{H}), 4.73(\mathrm{~d}, J=2.5 \mathrm{~Hz}, 2 \mathrm{H}), 2.48(\mathrm{t}, J=2.5 \mathrm{~Hz}, 1 \mathrm{H}) ;{ }^{13} \mathrm{C} \mathrm{NMR}(100$ $\left.\mathrm{MHz}, \mathrm{CDCl}_{3}\right): \delta 171.3,163.0(\mathrm{~d}, J=246.8 \mathrm{~Hz}), 136.6,133.7,133.6(\mathrm{~d}, J=3.3 \mathrm{~Hz}), 130.3(\mathrm{~d}$, $J=8.1 \mathrm{~Hz}), 129.9,129.0,115.8(\mathrm{~d}, J=21.6 \mathrm{~Hz}), 77.2,75.5,55.3,52.9$; HRMS (ESI TOF): Calculated for $\mathrm{C}_{17} \mathrm{H}_{13} \mathrm{ClFO}_{2}(\mathrm{M}+\mathrm{H})^{+}$: 303.0588, Found: 303.0595 . 
<smiles>C#CCOC(=O)C(c1ccc(Cl)cc1)c1ccc(Br)cc1</smiles>

Compound 3bc was synthesized following the general procedure (D). The product was obtained as a major product para-isomer $(p: o=90: 10)$, $p$ :o ratio determined by ${ }^{1} \mathrm{H}$ NMR. The product was obtained as viscous colourless liquid $(0.091 \mathrm{~g}, 50 \%$ yield $): R_{f}=0.45$ petroleum ether/EtOAc (95:5); IR (neat) $\mathrm{cm}^{-1}:$ 3294, 2946, 2130, 1740, 1591, 1488, 1369, 1301, 1183, 1142, 1088, 1006, 954, 807, 761, 681, 638; ${ }^{1} \mathrm{H}$ NMR (400 MHz, $\left.\mathrm{CDCl}_{3}\right): \delta 7.50$ - $7.43(\mathrm{~m}, 2 \mathrm{H}), 7.34-7.14(\mathrm{~m}, 6 \mathrm{H}), 4.98(\mathrm{~s}, 1 \mathrm{H}), 4.74(\mathrm{~d}, J=2.5 \mathrm{~Hz}, 2 \mathrm{H}), 2.49$ (t, $J=2.4$ $\mathrm{Hz}, 1 \mathrm{H}) ;{ }^{13} \mathrm{C} \mathrm{NMR}\left(100 \mathrm{MHz}, \mathrm{CDCl}_{3}\right): \delta 171.0,136.8,136.2,133.8,132.0,130.3,130.0$, 129.1, 121.9, 77.4, 75.6, 55.5, 53.0; HRMS (ESI TOF): Calculated for $\mathrm{C}_{17} \mathrm{H}_{13} \mathrm{BrClO}_{2}(\mathrm{M}+$ $\mathrm{H})^{+}: 362.9787$, Found: 362.9785 .

\section{Prop-2-yn-1-yl 2,2-bis(4-chlorophenyl)acetate (3cc):}<smiles>C#CCOC(=O)C(c1ccc(Cl)cc1)c1ccc(Cl)cc1</smiles>

Compound 3cc was synthesized following the general procedure (D). The product was obtained as a major product para-isomer $(p: o=89: 11)$, $p$ : o ratio determined by ${ }^{1} \mathrm{H}$ NMR. The product was obtained as colourless liquid $(0.083 \mathrm{~g}, 52 \%$ yield $): R_{f}=0.4$ petroleum ether/EtOAc (95:5); IR (neat) $\mathrm{cm}^{-1}:$ 3295, 2947, 2130, 1740, 1594, 1489, 1369, 1268, 1141, 1090, 1003, 954, 905, 808, 762, 681, 637; ${ }^{1} \mathrm{H}$ NMR (400 MHz, $\left.\mathrm{CDCl}_{3}\right): \delta 7.42-7.28(\mathrm{~m}$, 4H), $7.25-7.18(\mathrm{~m}, 4 \mathrm{H}), 5.00(\mathrm{~s}, 1 \mathrm{H}), 4.74(\mathrm{~d}, J=2.5 \mathrm{~Hz}, 2 \mathrm{H}), 2.48(\mathrm{t}, J=2.5 \mathrm{~Hz}, 1 \mathrm{H}) ;{ }^{13} \mathrm{C}$ NMR (100 MHz, $\left.\mathrm{CDCl}_{3}\right): \delta 171.1,136.3,133.8,130.0,129.1,77.2,75.6,55.4,53.0$; HRMS (ESI TOF): Calculated for $\mathrm{C}_{17} \mathrm{H}_{13} \mathrm{Cl}_{2} \mathrm{O}_{2}(\mathrm{M}+\mathrm{H})^{+}: 319.0293$, Found: 319.0305 . 


\section{Prop-2-yn-1-yl (S)-2-phenyl-2-(p-tolyl)acetate (3ad):}<smiles>C#CCOC(=O)C(c1ccccc1)c1ccc(C)cc1</smiles>

Compound 3ad was synthesized following the general procedure (D). The product was obtained as a major product para-isomer $(p: o=80: 20)$, $p$ : o ratio determined by ${ }^{1} \mathrm{H}$ NMR. The product was obtained as viscous colourless liquid $(0.111 \mathrm{~g}, 84 \%$ yield $): R_{f}=0.5$ petroleum ether/EtOAc (95:5); IR (neat) $\mathrm{cm}^{-1}$ : 3290, 2925, 2124, 1743, 1603, 1501, 1449, 1371, 1306, 1275, 1219, 1142, 999, 806, 732, 696, 640; ${ }^{1} \mathrm{H}$ NMR (400 MHz, $\left.\mathrm{CDCl}_{3}\right): \delta 7.45$ - $6.99(\mathrm{~m}, 9 \mathrm{H}), 5.03(\mathrm{~s}, 1 \mathrm{H}), 4.73(\mathrm{~d}, J=2.4 \mathrm{~Hz}, 2 \mathrm{H}), 2.46(\mathrm{t}, J=2.4 \mathrm{~Hz}, 1 \mathrm{H}), 2.31(\mathrm{~s}, 3 \mathrm{H})$; ${ }^{13} \mathrm{C} \mathrm{NMR}\left(100 \mathrm{MHz}, \mathrm{CDCl}_{3}\right): \delta 172.0,138.5,137.2,135.3,129.5,128.7,128.7,128.6,127.5$, 77.4, 75.3, 56.4, 52.7, 21.2; HRMS (ESI TOF): Calculated for $\mathrm{C}_{18} \mathrm{H}_{16} \mathrm{NaO}_{2}(\mathrm{M}+\mathrm{Na})^{+}$: 287.1048, Found: 287.1054 .

\section{Prop-2-yn-1-yl 2-(4-bromophenyl)-2-(p-tolyl)acetate (3bd):}<smiles>C#CCOC(=O)C(c1ccc(C)cc1)c1ccc(Br)cc1</smiles>

Compound 3bd was synthesized following the general procedure (D). The product was obtained as a major product para-isomer $(p: o=78: 22)$, $p$ :o ratio determined by ${ }^{1} \mathrm{H}$ NMR. The product was obtained as colourless liquid $\left(0.144 \mathrm{~g}, 84 \%\right.$ yield): $R_{f}=0.45$ petroleum ether/EtOAc (95:5); IR (neat) $\mathrm{cm}^{-1}:$ 3291, 3025, 2945, 2130, 1739, 1592, 1487, 1441, 1369, 1270, 1219, 1073, 1002, 951, 809, 754, 680, 638; ${ }^{1} \mathrm{H}$ NMR (400 MHz, $\left.\mathrm{CDCl}_{3}\right): \delta 7.51-7.37$ (m, 2H), $7.28-7.03(\mathrm{~m}, 6 \mathrm{H}), 4.98(\mathrm{~s}, 1 \mathrm{H}), 4.82-4.65(\mathrm{~m}, 2 \mathrm{H}), 2.46(\mathrm{t}, J=2.5 \mathrm{~Hz}, 1 \mathrm{H}), 2.32$ (s, 3H); ${ }^{13} \mathrm{C}$ NMR $\left(100 \mathrm{MHz}, \mathrm{CDCl}_{3}\right): \delta 171.5,137.6137 .5,134.8,131.8,130.4,129.6$, 128.4, 121.6, 77.4, 75.4, 55.8, 52.8, 21.2; HRMS (ESI TOF): Calculated for $\mathrm{C}_{18} \mathrm{H}_{16} \mathrm{BrO}_{2}(\mathrm{M}$ $+\mathrm{H})^{+}$: 343.0334, Found: 343.0330 . 


\section{Prop-2-yn-1-yl 2-(3,4-dimethylphenyl)-2-phenylacetate (3ae):}<smiles>C#CCOC(=O)C(c1ccccc1)c1ccc(C)c(C)c1</smiles>

Compound 3ae was synthesized following the general procedure (D). The product was obtained as a major product para-isomer $(p: o=84: 16)$, $p$ :o ratio determined by ${ }^{1} \mathrm{H}$ NMR. The product was obtained as white solid $(0.124 \mathrm{~g}, 89 \%$ yield $): R_{f}=0.5$ petroleum ether/EtOAc (95:5); m.p.: $88-90{ }^{\circ} \mathrm{C}$; IR (neat) $\mathrm{cm}^{-1}: 3287,3027,2934,2126,1740,1601$, 1497, 1448, 1371, 1207, 1137, 1079, 997, 815, 695, 637, 573; ${ }^{1} \mathrm{H}$ NMR (400 MHz, $\left.\mathrm{CDCl}_{3}\right): \delta$ $7.33-7.21(\mathrm{~m}, 5 \mathrm{H}), 7.11-7.02(\mathrm{~m}, 3 \mathrm{H}), 5.01(\mathrm{~s}, 1 \mathrm{H}), 4.79-4.68(\mathrm{~m}, 2 \mathrm{H}), 2.46(\mathrm{t}, J=2.5$ $\mathrm{Hz}, 1 \mathrm{H}), 2.30-2.15(\mathrm{~m}, 6 \mathrm{H})\left(\right.$ for $\left.2-\mathrm{CH}_{3}\right) ;{ }^{13} \mathrm{C} \mathrm{NMR}\left(100 \mathrm{MHz}, \mathrm{CDCl}_{3}\right): \delta 172.0,138.6$, 137.0, 135.9, 135.7, 130.0, 129.9, 128.7, 128.7, 127.4, 126.0, 77.6, 75.2, 56.4, 52.7, 20.0, 19.5; HRMS (ESI TOF): Calculated for $\mathrm{C}_{19} \mathrm{H}_{19} \mathrm{O}_{2}(\mathrm{M}+\mathrm{H})^{+}:$279.1385, Found: 279.1387.

\section{Prop-2-yn-1-yl 2-(4-bromophenyl)-2-(3,4-dimethylphenyl)acetate (3be):}<smiles>C#CCOC(=O)C(c1ccc(Br)cc1)c1ccc(C)c(C)c1</smiles>

Compound 3be was synthesized following the general procedure (D). The product was obtained as a major product para-isomer $(p: o=83: 17)$, $p$ : o ratio determined by ${ }^{1} \mathrm{H}$ NMR. The product was obtained as colourless liquid $(0.161 \mathrm{~g}, 90 \%$ yield $): R_{f}=0.5$ petroleum ether/EtOAc (95:5); IR (neat) $\mathrm{cm}^{-1}:$ 3293, 2939, 2123, 1744, 1590, 1490, 1446, 1371, 1272 , 1145, 1073, 1007, 949, 818, 762, 681, 641, 568; ${ }^{1} \mathrm{H}$ NMR (400 MHz, $\left.\mathrm{CDCl}_{3}\right): \delta 7.48-7.39$ (m, 2H), $7.21-6.99(\mathrm{~m}, 5 \mathrm{H}), 4.95(\mathrm{~s}, 1 \mathrm{H}), 4.79-4.66(\mathrm{~m}, 2 \mathrm{H}), 2.52-2.41(\mathrm{~m}, 1 \mathrm{H}), 2.29-$ $2.12(\mathrm{~m}, 6 \mathrm{H}) ;{ }^{13} \mathrm{C}$ NMR $\left(100 \mathrm{MHz}, \mathrm{CDCl}_{3}\right): \delta 171.6,137.6,137.2,136.2,135.2,131.8$, $130.4,130.1,129.7,125.9,121.5,77.4,75.4,55.8,52.8,20.0,19.5$; HRMS (ESI TOF): Calculated for $\mathrm{C}_{19} \mathrm{H}_{18} \mathrm{BrO}_{2}(\mathrm{M}+\mathrm{H})^{+}: 357.0490$, Found: 357.0487 . 
Prop-2-yn-1-yl 2-(2,5-dimethylphenyl)-2-phenylacetate (3af):<smiles>C#CCOC(=O)C(c1ccccc1)c1cc(C)ccc1C</smiles>

Compound 3af was synthesized following the general procedure (D). The product was obtained as white solid ( $0.12 \mathrm{~g}, 86 \%$ yield): $R_{f}=0.5$ petroleum ether/EtOAc (95:5); m.p.: 79$81{ }^{\circ} \mathrm{C}$; IR (neat) $\mathrm{cm}^{-1}: 3289,2929,2126,1740,1606,1498,1447,1370,1273,1213,1141$, 996, 946, 812, 736, 692, 637, 578; ${ }^{1} \mathrm{H}$ NMR (400 MHz, $\left.\mathrm{CDCl}_{3}\right): \delta 7.51-7.14(\mathrm{~m}, 5 \mathrm{H}), 7.09$ - $6.95(\mathrm{~m}, 3 \mathrm{H}), 5.22(\mathrm{~s}, 1 \mathrm{H}), 4.74(\mathrm{~d}, J=2.5 \mathrm{~Hz}, 2 \mathrm{H}), 2.46(\mathrm{t}, J=2.5 \mathrm{~Hz}, 1 \mathrm{H}), 2.28(\mathrm{~s}, 3 \mathrm{H})$, $2.23(\mathrm{~s}, 3 \mathrm{H}) ;{ }^{13} \mathrm{C} \mathrm{NMR}\left(100 \mathrm{MHz}, \mathrm{CDCl}_{3}\right): \delta 172.2,137.6,136.3,135.8,133.4,130.7,129.1$, 128.8, 128.7, 128.3, 127.4, 77.6, 75.2, 53.5, 52.7, 21.3, 19.5; HRMS (ESI TOF): Calculated for $\mathrm{C}_{19} \mathrm{H}_{18} \mathrm{NaO}_{2}(\mathrm{M}+\mathrm{Na})^{+}:$301.1204, Found: 301.1209 .

Prop-2-yn-1-yl 2-(4-bromophenyl)-2-(2,5-dimethylphenyl)acetate (3bf):<smiles>C#CCOC(=O)C(c1ccc(Br)cc1)c1cc(C)ccc1C</smiles>

Compound 3bf was synthesized following the general procedure (D). The product was obtained as white solid (0.168 g, 94\% yield): $R_{f}=0.5$ petroleum ether/EtOAc (95:5); m.p.: $115-117{ }^{\circ} \mathrm{C}$; IR (neat) $\mathrm{cm}^{-1}: 3292,2930,2123,1743,1613,1491,1445,1370,1315$, 1213, 1149, 1074, 1007, 813, 761, 679, 641; ${ }^{1} \mathrm{H}$ NMR (400 MHz, $\left.\mathrm{CDCl}_{3}\right): \delta 7.61-7.33(\mathrm{~m}$, 2H), $7.19-6.94(\mathrm{~m}, 5 \mathrm{H}), 5.16(\mathrm{~s}, 1 \mathrm{H}), 4.90-4.64(\mathrm{~m}, 2 \mathrm{H}), 2.48(\mathrm{t}, J=2.5 \mathrm{~Hz}, 1 \mathrm{H}), 2.30(\mathrm{~s}$, $3 \mathrm{H}), 2.21(\mathrm{~s}, 3 \mathrm{H}) ;{ }^{13} \mathrm{C} \mathrm{NMR}\left(\left(100 \mathrm{MHz}, \mathrm{CDCl}_{3}\right): \delta 171.8,136.7,136.1,135.8,133.3,131.8\right.$, $130.9,130.8,128.59,128.58,121.5,77.4,75.3,53.0,52.8,21.2,19.4$; HRMS (ESI TOF): Calculated for $\mathrm{C}_{19} \mathrm{H}_{17} \mathrm{BrNaO}_{2}(\mathrm{M}+\mathrm{Na})^{+}$: 379.0310 , Found: 379.0305 . 


\section{Prop-2-yn-1-yl 2-mesityl-2-phenylacetate (3ag):}<smiles>C#CCOC(=O)C(c1ccccc1)c1c(C)cc(C)cc1C</smiles>

Compound 3ag was synthesized following the general procedure (D). The product was obtained as colourless liquid $(0.135 \mathrm{~g}, 92 \%$ yield $): R_{f}=0.5$ petroleum ether/EtOAc (95:5); IR (neat) $\mathrm{cm}^{-1}: 3286,2922,2125,1742,1608,1489,1448,1371,1271,1154,1000$, 943, 853, 734, 693, 569; ${ }^{1} \mathrm{H}$ NMR (400 MHz, $\left.\mathrm{CDCl}_{3}\right)$ : $\delta 7.33-7.20$ (m, 3H), $7.15-7.08$ (m, 2H), $6.90(\mathrm{~s}, 2 \mathrm{H}), 5.42(\mathrm{~s}, 1 \mathrm{H}), 4.79-4.68(\mathrm{~m}, 2 \mathrm{H}), 2.43(\mathrm{t}, J=2.5 \mathrm{~Hz}, 1 \mathrm{H}), 2.29(\mathrm{~s}, 3 \mathrm{H}), 2.18$ $(\mathrm{s}, 6 \mathrm{H}) ;{ }^{13} \mathrm{C} \mathrm{NMR}\left(100 \mathrm{MHz}, \mathrm{CDCl}_{3}\right): \delta 172.6,137.6,137.1,136.5,131.9,130.1,128.8$, 128.3, 127.0, 77.8, 75.0, 52.5, 50.8, 21.0 (for two type of carbons); HRMS (ESI TOF): Calculated for $\mathrm{C}_{20} \mathrm{H}_{20} \mathrm{NaO}_{2}(\mathrm{M}+\mathrm{Na})^{+}: 315.1361$, Found: 315.1365 .

\section{Prop-2-yn-1-yl 2-(4-bromophenyl)-2-mesitylacetate (3bg):}<smiles>C#CCOC(=O)C(c1ccc(Br)cc1)c1c(C)cc(C)cc1C</smiles>

Compound $3 \mathbf{b g}$ was synthesized following the general procedure (D). The product was obtained as colourless liquid $(0.182 \mathrm{~g}, 98 \%$ yield $): R_{f}=0.45$ petroleum ether/EtOAc (95:5); IR (neat) $\mathrm{cm}^{-1}: 3292,2949,2120,1743,1611,1486,1447,1159,1075,1005,946$, 878, 778, 679, 634; ${ }^{1} \mathrm{H}$ NMR (400 MHz, $\left.\mathrm{CDCl}_{3}\right): \delta 7.45-7.35(\mathrm{~m}, 2 \mathrm{H}), 7.06-6.97$ (m, 2H), $6.91(\mathrm{~s}, 2 \mathrm{H}), 5.33(\mathrm{~s}, 1 \mathrm{H}), 4.89-4.57(\mathrm{~m}, 2 \mathrm{H}), 2.44(\mathrm{t}, J=2.4 \mathrm{~Hz}, 1 \mathrm{H}), 2.30(\mathrm{~s}, 3 \mathrm{H}), 2.16(\mathrm{~s}$, $6 \mathrm{H}) ;{ }^{13} \mathrm{C} \mathrm{NMR}\left(100 \mathrm{MHz}, \mathrm{CDCl}_{3}\right): \delta 172.2,137.5,137.4,135.5,131.4,131.4,130.6,130.1$, 121.1, 77.6, 75.2, 52.6, 50.1, 21.0, 20.9; HRMS (ESI TOF): Calculated for $\mathrm{C}_{20} \mathrm{H}_{19} \mathrm{BrNaO}_{2}(\mathrm{M}$ $+\mathrm{Na})^{+}:$393.0466, Found: 393.0452. 


\section{Prop-2-yn-1-yl 2-(4-chlorophenyl)-2-mesitylacetate (3cg):}<smiles>C#CCOC(=O)C(c1ccc(Cl)cc1)c1c(C)cc(C)cc1C</smiles>

Compound $3 \mathrm{cg}$ was synthesized following the general procedure (D). The product was obtained as colourless liquid $\left(0.154 \mathrm{~g}, 94 \%\right.$ yield): $R_{f}=0.4$ petroleum ether/EtOAc (95:5); IR (neat) $\mathrm{cm}^{-1}: 3293,2923,2123,1742,1610,1489,1448,1268,1159,1093,1008$, 848, 775, 681, 635; ${ }^{1} \mathrm{H}$ NMR (400 MHz, $\left.\mathrm{CDCl}_{3}\right): \delta 7.29-7.21(\mathrm{~m}, 2 \mathrm{H}), 7.10-7.03(\mathrm{~m}, 2 \mathrm{H})$, $6.91(\mathrm{~s}, 2 \mathrm{H}), 5.35(\mathrm{~s}, 1 \mathrm{H}), 4.78-4.68(\mathrm{~m}, 2 \mathrm{H}), 2.43(\mathrm{t}, J=2.5 \mathrm{~Hz}, 1 \mathrm{H}), 2.30(\mathrm{~s}, 6 \mathrm{H}), 2.16(\mathrm{~s}$, $3 \mathrm{H}) ;{ }^{13} \mathrm{C} \mathrm{NMR}\left(100 \mathrm{MHz}, \mathrm{CDCl}_{3}\right): \delta 172.2,137.5,137.3,135.0,132.9,131.5,130.2,130.1$, 128.5, 77.6, 75.1, 52.6, 50.1, 21.0, 20.9; HRMS (ESI TOF): Calculated for $\mathrm{C}_{20} \mathrm{H}_{19} \mathrm{ClNaO}_{2}(\mathrm{M}$ $+\mathrm{Na})^{+}: 349.0971$, Found: 349.0966.

\section{Prop-2-yn-1-yl 2-(4-fluorophenyl)-2-mesitylacetate (3dg):}<smiles>C#CCOC(=O)C(c1ccc(F)cc1)c1c(C)cc(C)cc1C</smiles>

Compound 3dg was synthesized following the general procedure (D). The product was obtained as colourless liquid $(0.148 \mathrm{~g}, 95 \%$ yield $): R_{f}=0.45$ petroleum ether/EtOAc (95:5); IR (neat) $\mathrm{cm}^{-1}: 3293,2924,2122,1743,1606,1509,1452,1372,1273,1227$, 1158,1004, 847, 799, 681, 639; ${ }^{1} \mathrm{H}$ NMR (400 MHz, $\left.\mathrm{CDCl}_{3}\right): \delta 7.14-7.07$ (m, 2H), 7.02 $6.94(\mathrm{~m}, 2 \mathrm{H}), 6.92(\mathrm{~s}, 2 \mathrm{H}), 5.36(\mathrm{~s}, 1 \mathrm{H}), 4.83-4.64(\mathrm{~m}, 2 \mathrm{H}), 2.44(\mathrm{t}, J=2.5 \mathrm{~Hz}, 1 \mathrm{H}), 2.31(\mathrm{~s}$, 3H), $2.18(\mathrm{~s}, 6 \mathrm{H}) ;{ }^{13} \mathrm{C} \mathrm{NMR}\left(100 \mathrm{MHz}, \mathrm{CDCl}_{3}\right): \delta 172.5,161.9(\mathrm{~d}, J=245.6 \mathrm{~Hz}), 137.5$, 137.3, 132.1 (d, $J=3.2 \mathrm{~Hz}), 131.8,130.4$ (d, $J=7.9 \mathrm{~Hz}), 130.1,115.1$ (d, $J=21.4 \mathrm{~Hz}), 77.7$, 75.1, 52.6, 50.0, 21.0, 20.9; HRMS (ESI TOF): Calculated for $\mathrm{C}_{18} \mathrm{H}_{19} \mathrm{FNaO}_{2}(\mathrm{M}+\mathrm{Na})^{+}$: 333.1267, Found: 333.1268 . 


\section{Prop-2-yn-1-yl 2-(4-bromophenyl)-2-(4-methoxyphenyl)acetate (3bh):}<smiles>C#CCOC(=O)C(c1ccc(Br)cc1)c1ccc(OC)cc1</smiles>

Compound 3bh was synthesized following the general procedure (D). The product was obtained as a major product para-isomer $(p: o=74: 26)$, $p$ :o ratio determined by ${ }^{1} \mathrm{H}$ NMR. The product was obtained as ( $0.169 \mathrm{~g}, 94 \%$ yield). $R_{f}=0.3$ petroleum ether/EtOAc (95:5); IR (neat) $\mathrm{cm}^{-1}: 3290,2946,2838,2309,2126,1899,1739,1504,1368,1297,1147,1007,815$, 681; ${ }^{1} \mathrm{H}$ NMR (400 MHz, $\left.\mathrm{CDCl}_{3}\right): \delta 7.51$ - 7.38 (m, 2H), 7.31 - 7.12 (m, 4H), 6.94 - 6.80 $(\mathrm{m}, 2 \mathrm{H}), 4.96(\mathrm{~s}, 1 \mathrm{H}), 4.78-4.65(\mathrm{~m}, 2 \mathrm{H}), 3.78(\mathrm{~s}, 3 \mathrm{H}), 2.46(\mathrm{t}, J=2.5 \mathrm{~Hz}, 1 \mathrm{H}) ;{ }^{13} \mathrm{C}$ NMR $\left(100 \mathrm{MHz}, \mathrm{CDCl}_{3}\right): \delta 171.6,159.1,137.7,131.8,131.0,130.3,129.7,121.6,114.3,77.4$, 75.4, 55.4, 55.3, 52.8; HRMS (ESI TOF): Calculated for $\mathrm{C}_{18} \mathrm{H}_{16} \mathrm{BrO}_{3}(\mathrm{M}+\mathrm{H})^{+}:$359.0283, Found: 359.0275.

Prop-2-yn-1-yl 2-(2,5-dimethoxyphenyl)-2-phenylacetate (3ai):<smiles>C#CCOC(=O)C(c1ccccc1)c1cc(OC)ccc1OC</smiles>

Compound 3ai was synthesized following the general procedure (D). The product was obtained as white solid ( $0.149 \mathrm{~g}, 96 \%$ yield): $R_{f}=0.4$ petroleum ether/EtOAc (80:20); m.p.: $74-76{ }^{\circ} \mathrm{C}$; IR (neat) $\mathrm{cm}^{-1}: 3286,2945,2836,2126,1741,1595,1496,1455,1369,1277,1219$, 1145, 1026, 947, 807, 699, 640; ${ }^{1} \mathrm{H}$ NMR (400 MHz, $\left.\mathrm{CDCl}_{3}\right): \delta 7.37-7.24(\mathrm{~m}, 5 \mathrm{H}), 6.82-$ $6.74(\mathrm{~m}, 2 \mathrm{H}), 6.61(\mathrm{~d}, J=2.9 \mathrm{~Hz}, 1 \mathrm{H}), 5.30(\mathrm{~s}, 1 \mathrm{H}), 4.79-4.66(\mathrm{~m}, 2 \mathrm{H}), 3.78(\mathrm{~s}, 3 \mathrm{H}), 3.68(\mathrm{~s}$, $3 \mathrm{H}), 2.44(\mathrm{t}, J=2.5 \mathrm{~Hz}, 1 \mathrm{H}) ;{ }^{13} \mathrm{C} \mathrm{NMR}\left(100 \mathrm{MHz}, \mathrm{CDCl}_{3}\right): \delta 172.1,153.6,151.3,137.0$, $129.2,128.8,128.6,127.6,116.2,112.5,111.4,77.9,74.9,56.2,55.7,52.5,51.0$; HRMS (ESI TOF): Calculated for $\mathrm{C}_{19} \mathrm{H}_{19} \mathrm{O}_{4}(\mathrm{M}+\mathrm{H})^{+}:$311.1283, Found: 311.1277. 
<smiles>C#CCOC(=O)C(c1ccc(Br)cc1)c1cc(OC)ccc1OC</smiles>

Compound $\mathbf{3 b i}$ was synthesized following the general procedure (D). The product was obtained as white solid ( $0.175 \mathrm{~g}, 90 \%$ yield): $R_{f}=0.45$ petroleum ether/EtOAc (80:20); m.p.: 97-99 ${ }^{\circ} \mathrm{C}$; IR (neat) $\mathrm{cm}^{-1}: 3289,2944,2837,2126,1741,1593,1494,1222,1148,1010,945$, 806, 712, 641, 537; ${ }^{1} \mathrm{H}$ NMR (400 MHz, $\left.\mathrm{CDCl}_{3}\right): \delta 7.49-7.42(\mathrm{~m}, 2 \mathrm{H}), 7.23-7.17(\mathrm{~m}, 2 \mathrm{H})$, $6.86-6.73(\mathrm{~m}, 2 \mathrm{H}), 6.62(\mathrm{~d}, J=2.8 \mathrm{~Hz}, 1 \mathrm{H}), 5.25(\mathrm{~s}, 1 \mathrm{H}), 4.78-4.67(\mathrm{~m}, 2 \mathrm{H}), 3.77(\mathrm{~s}, 3 \mathrm{H})$, $3.70(\mathrm{~s}, 3 \mathrm{H}), 2.45$ (t, $J=2.5 \mathrm{~Hz}, 1 \mathrm{H}) ;{ }^{13} \mathrm{C} \mathrm{NMR}\left(100 \mathrm{MHz}, \mathrm{CDCl}_{3}\right): \delta$ 171.7, 153.6, 151.1, $136.3,131.9,130.9,128.0,121.6,115.9,112.7,111.5,77.7,75.1,56.2,55.8,52.6,50.4$; HRMS (ESI TOF): Calculated for $\mathrm{C}_{19} \mathrm{H}_{17} \mathrm{BrO}_{4}(\mathrm{M})^{+}: 388.0310$, Found: 388.0305 .

Prop-2-yn-1-yl 2-(4-chlorophenyl)-2-(2,5-dimethoxyphenyl)acetate (3ci):<smiles>C#CCOC(=O)C(c1ccc(Cl)cc1)c1cc(OC)ccc1OC</smiles>

Compound 3ci was synthesized following the general procedure (D). The product was obtained as white solid ( $0.159 \mathrm{~g}, 92 \%$ yield): $R_{f}=0.45$ petroleum ether/EtOAc (80:20); m.p.: 98-99 ${ }^{\circ} \mathrm{C}$; IR (neat) $\mathrm{cm}^{-1}: 3290,2947,2836,2125,1741,1595,1494,1278,1222,1174,1095$, 1014, 806, 755, 709, 639, 539; ${ }^{1} \mathrm{H}$ NMR (400 MHz, $\left.\mathrm{CDCl}_{3}\right): \delta 7.33-7.28(\mathrm{~m}, 2 \mathrm{H}), 7.27$ $7.22(\mathrm{~m}, 2 \mathrm{H}), 6.85-6.74(\mathrm{~m}, 2 \mathrm{H}), 6.62(\mathrm{~d}, J=2.8 \mathrm{~Hz}, 1 \mathrm{H}), 5.27(\mathrm{~s}, 1 \mathrm{H}), 4.78-4.67(\mathrm{~m}, 2 \mathrm{H})$, 3.77 (s, 3H), 3.70 (s, 3H), 2.45 (t, $\left.J=2.5 \mathrm{~Hz}, 1 \mathrm{H}) ;{ }^{13} \mathrm{C} \mathrm{NMR} \mathrm{(100} \mathrm{MHz,} \mathrm{CDCl}_{3}\right): \delta 171.8$, $153.7,151.2,135.8,133.5,130.6,128.9,128.1,115.9,112.8,111.6,77.7,75.0,56.2$, 55.8, 52.6, 50.3; HRMS (ESI TOF): Calculated for $\mathrm{C}_{19} \mathrm{H}_{17} \mathrm{ClNaO}_{4}(\mathrm{M}+\mathrm{Na})^{+}$: 367.0713, Found: 367.0710 . 


\section{Prop-2-yn-1-yl 2-(2,5-dimethoxyphenyl)-2-(4-fluorophenyl)acetate (3di):}<smiles>C#CCOC(=O)C(c1ccc(F)cc1)c1cc(OC)ccc1OC</smiles>

Compound 3di was synthesized following the general procedure (D). The product was obtained as colourless liquid ( $0.156 \mathrm{~g}, 95 \%$ yield): $R_{f}=0.35$ petroleum ether/EtOAc $(85: 15)$; IR (neat) $\mathrm{cm}^{-1}: 3291,2948,2838,2122,1744,1603,1503,1457,1369,1280,1226,1153$, 1040, 844, 808, 712, 642; ${ }^{1} \mathrm{H}$ NMR (400 MHz, $\left.\mathrm{CDCl}_{3}\right): \delta 7.34-7.25(\mathrm{~m}, 2 \mathrm{H}), 7.07-6.96$ $(\mathrm{m}, 2 \mathrm{H}), 6.78(\mathrm{dt}, J=8.8,5.8 \mathrm{~Hz}, 2 \mathrm{H}), 6.61(\mathrm{~d}, J=2.8 \mathrm{~Hz}, 1 \mathrm{H}), 5.27(\mathrm{~s}, 1 \mathrm{H}), 4.78-4.66(\mathrm{~m}$, 2H), 3.77 (s, 3H), 3.69 (s, 3H), 2.45 (t, $J=2.5 \mathrm{~Hz}, 1 \mathrm{H}) ;{ }^{13} \mathrm{C}$ NMR (100 MHz, $\left.\mathrm{CDCl}_{3}\right): \delta$ 172.0, 162.2 (d, $J=245.8 \mathrm{~Hz}), 153.6,151.1,132.9$ (d, $J=3.2 \mathrm{~Hz}), 130.8$ (d, $J=8.2 \mathrm{~Hz})$, 128.4, 115.8 (d, $J=18.1 \mathrm{~Hz}), 115.5,112.6,111.5,77.7,75.0,56.1,55.7,52.5,50.2$; HRMS (ESI TOF): Calculated for $\mathrm{C}_{19} \mathrm{H}_{17} \mathrm{FNaO}_{4}(\mathrm{M}+\mathrm{Na})^{+}$: 351.1009, Found: 351.1000 .

Prop-2-yn-1-yl 2-(2,5-dimethoxyphenyl)-2-(naphthalen-2-yl)acetate (3gi):<smiles>C#CCOC(=O)C(c1ccc2ccccc2c1)c1cc(OC)ccc1OC</smiles>

Compound 3gi was synthesized following the general procedure (D). The product was obtained as white solid ( $0.151 \mathrm{~g}, 84 \%$ yield): $R_{f}=0.45$ petroleum ether/EtOAc (80:20); m.p.: 91-93 ${ }^{\circ} \mathrm{C}$; IR (neat) $\mathrm{cm}^{-1}: 3287,2945,2837,2125,1740,1597,1497,1455,1369,1222,1148$, 1032, 863, 808, 750, 709, 644; ${ }^{1} \mathrm{H}$ NMR (400 MHz, $\left.\mathrm{CDCl}_{3}\right): \delta 7.87-7.73(\mathrm{~m}, 4 \mathrm{H}), 7.52$ $7.40(\mathrm{~m}, 3 \mathrm{H}), 6.84-6.75(\mathrm{~m}, 2 \mathrm{H}), 6.63(\mathrm{~d}, J=3.0 \mathrm{~Hz}, 1 \mathrm{H}), 5.47(\mathrm{~s}, 1 \mathrm{H}), 4.82-4.69(\mathrm{~m}, 2 \mathrm{H})$, $3.80(\mathrm{~s}, 3 \mathrm{H}), 3.65(\mathrm{~s}, 3 \mathrm{H}), 2.45(\mathrm{t}, J=2.5 \mathrm{~Hz}, 1 \mathrm{H}) ;{ }^{13} \mathrm{C} \mathrm{NMR}\left(100 \mathrm{MHz}, \mathrm{CDCl}_{3}\right): \delta 172.1$, $153.6,151.3$, 134.5, 133.6, 132.8, 128.6, 128.5, 128.1, 128.0, 127.7, 127.3, 126.3, 126.1, 116.3, 112.6, 111.4, 77.9, 75.0, 56.2, 55.8, 52.6, 51.1; HRMS (ESI TOF): Calculated for $\mathrm{C}_{23} \mathrm{H}_{20} \mathrm{NaO}_{4}(\mathrm{M}+\mathrm{Na})^{+}:$383.1259, Found: 383.1262 . 


\section{Prop-2-yn-1-yl 2-phenyl-2-(2,4,6-trimethoxyphenyl)acetate (3aj):}<smiles>C#CCOC(=O)C(c1ccccc1)c1c(OC)cc(OC)cc1OC</smiles>

Compound 3aj was synthesized following the general procedure (D). The product was obtained as white solid $(0.157 \mathrm{~g}, 92 \%$ yield $): R_{f}=0.35$ petroleum ether/EtOAc (80:20); m.p.: 121-123 ${ }^{\circ} \mathrm{C}$; IR (neat) $\mathrm{cm}^{-1}: 3281,2937,2842,2127,1741,1597,1496,1420,1337,1195$, 1114, 1002, 949, 864, 814, 746, 696, 664; ${ }^{1} \mathrm{H}$ NMR ((400 MHz, $\left.\left.\mathrm{CDCl}_{3}\right)\right)$ : $\delta 7.34-7.30(\mathrm{~m}$, 2H), $7.28-7.23(\mathrm{~m}, 2 \mathrm{H}), 7.22-7.16(\mathrm{~m}, 1 \mathrm{H}), 6.14(\mathrm{~s}, 2 \mathrm{H}), 5.33(\mathrm{~s}, 1 \mathrm{H}), 4.77-4.64(\mathrm{~m}, 2 \mathrm{H})$, $3.80(\mathrm{~s}, 3 \mathrm{H}), 3.78(\mathrm{~s}, 6 \mathrm{H}), 2.38(\mathrm{t}, J=2.4 \mathrm{~Hz}, 1 \mathrm{H}) ;{ }^{13} \mathrm{C} \mathrm{NMR}\left(100 \mathrm{MHz}, \mathrm{CDCl}_{3}\right): \delta 173.1$, $160.7,158.2,138.7,129.4,128.1,126.8,109.2,90.9,78.5,74.2,55.9,55.4,52.0,45.8$; HRMS (ESI TOF): Calculated for $\mathrm{C}_{20} \mathrm{H}_{21} \mathrm{O}_{5}(\mathrm{M}+\mathrm{H})^{+}: 341.1389$, Found: 341.1398 .

\section{Prop-2-yn-1-yl 2-(4-bromophenyl)-2-(2,4,6-trimethoxyphenyl)acetate (3bj):}<smiles>C#CCOC(=O)C(c1ccc(Br)cc1)c1c(OC)cc(OC)cc1OC</smiles>

Compound 3bj was synthesized following the general procedure (D). The product was obtained as colourless liquid ( $0.176 \mathrm{~g}, 84 \%$ yield): $R_{f}=0.3$ petroleum ether/EtOAc (80:20); IR(neat) $\mathrm{cm}^{-1}: 3287,2943,2841,2121,1743,1600,1460,1420,1195,1157,1061$, 1009, 950, 814, 759, 668, 634; ${ }^{1} \mathrm{H}$ NMR (400 MHz, $\left.\mathrm{CDCl}_{3}\right): \delta 7.41-7.33(\mathrm{~m}, 2 \mathrm{H}), 7.26-$ $7.17(\mathrm{~m}, 2 \mathrm{H}), 6.13(\mathrm{~s}, 2 \mathrm{H}), 5.27(\mathrm{~s}, 1 \mathrm{H}), 4.75-4.64(\mathrm{~m}, 2 \mathrm{H}), 3.79(\mathrm{~s}, 3 \mathrm{H}), 3.77(\mathrm{~s}, 6 \mathrm{H}), 2.39$ $(\mathrm{t}, J=2.5 \mathrm{~Hz}, 1 \mathrm{H}) ;{ }^{13} \mathrm{C} \mathrm{NMR}\left(100 \mathrm{MHz}, \mathrm{CDCl}_{3}\right): \delta 172.6,160.8,158.1,137.8,131.2,131.1$, 120.7, 108.6, 90.9, 78.3, 74.4, 55.8, 55.4, 52.1, 45.2; HRMS (ESI TOF): Calculated for $\mathrm{C}_{20} \mathrm{H}_{20} \mathrm{BrO}_{5}(\mathrm{M}+\mathrm{H})^{+}:$419.0494, Found: 419.0494 . 
<smiles>C#CCOC(=O)C(c1ccc2ccccc2c1)c1c(OC)cc(OC)cc1OC</smiles>

Compound 3gj was synthesized following the general procedure (D). The product was obtained as white solid ( $0.166 \mathrm{~g}, 85 \%$ yield): $R_{f}=0.25$ petroleum ether/EtOAc (80:20); m.p.: 99-101 ${ }^{\circ} \mathrm{C}$; IR (neat) $\mathrm{cm}^{-1}: 3285,2941,2842,2122,1741,1598,1499,1458,1422,1154$, 1115, 1026, 950, 857, 812, 751, 662; ${ }^{1} \mathrm{H}$ NMR (400 MHz, $\left.\mathrm{CDCl}_{3}\right): \delta 7.80-7.72(\mathrm{~m}, 3 \mathrm{H})$, $7.67(\mathrm{~s}, 1 \mathrm{H}), 7.55(\mathrm{dd}, J=8.5,1.8 \mathrm{~Hz}, 1 \mathrm{H}), 7.42-7.36(\mathrm{~m}, 2 \mathrm{H}), 6.16(\mathrm{~s}, 2 \mathrm{H}), 5.50(\mathrm{~s}, 1 \mathrm{H})$, $4.82-4.66(\mathrm{~m}, 2 \mathrm{H}), 3.81(\mathrm{~s}, 3 \mathrm{H}), 3.80(\mathrm{~s}, 6 \mathrm{H}), 2.40(\mathrm{t}, J=2.4 \mathrm{~Hz}, 1 \mathrm{H}) ;{ }^{13} \mathrm{C} \mathrm{NMR}(100 \mathrm{MHz}$, $\left.\mathrm{CDCl}_{3}\right): \delta 173.1,160.8,158.3,136.3,133.4,132.6,128.1,128.0,127.9,127.6,125.6,125.5$, 109.0, 91.0, 78.5, 77.4, 74.3, 55.9, 55.5, 52.1, 46.0; HRMS (ESI TOF): Calculated for $\mathrm{C}_{24} \mathrm{H}_{23} \mathrm{O}_{5}(\mathrm{M}+\mathrm{H})^{+}:$391.1545, Found: 391.1546 .

\section{Prop-2-yn-1-yl 2-(benzo[d][1,3]dioxol-5-yl)-2-phenylacetate (3ak):}<smiles>C#CCOC(=O)C(c1ccccc1)c1ccc2c(c1)OCO2</smiles>

Compound 3ak was synthesized following the general procedure (D). The product was obtained as a major product para-isomer $(p: o=90: 10)$, $p$ :o ratio determined by ${ }^{1} \mathrm{H}$ NMR. The product was obtained as colourless liquid $(0.138 \mathrm{~g}, 94 \%$ yield $): R_{f}=0.55$ petroleum ether/EtOAc (80:20); IR(neat) $\mathrm{cm}^{-1}: 3288,3027,2895,2126,1739,1490,1444,1367,1239$, 1138, 1032, 997, 930, 807, 735, 694, 643; ${ }^{1} \mathrm{H}$ NMR (400 MHz, $\left.\mathrm{CDCl}_{3}\right): \delta 7.36-7.25$ (m, $5 \mathrm{H}), 6.85-6.71(\mathrm{~m}, 3 \mathrm{H}), 5.93(\mathrm{~d}, J=3.9 \mathrm{~Hz}, 2 \mathrm{H}), 4.98(\mathrm{~s}, 1 \mathrm{H}), 4.73(\mathrm{~d}, J=2.6 \mathrm{~Hz}, 2 \mathrm{H})$, $2.50-2.43(\mathrm{~m}, 1 \mathrm{H}) ;{ }^{13} \mathrm{C} \mathrm{NMR}\left(100 \mathrm{MHz}, \mathrm{CDCl}_{3}\right): \delta 171.8,148.0,147.1,138.4,132.1$, 128.8, 128.5, 127.6, 122.1, 109.3, 108.4, 101.3, 77.4, 75.4, 56.3, 52.8; HRMS (ESI TOF): Calculated for $\mathrm{C}_{18} \mathrm{H}_{14} \mathrm{NaO}_{4}(\mathrm{M}+\mathrm{Na})^{+}: 317.0790$, Found: 317.0778 . 
<smiles>C#CCOC(=O)C(c1ccc(Br)cc1)c1ccc2c(c1)OCO2</smiles>

Compound 3bk was synthesized following the general procedure (D). The product was obtained as a major product para-isomer $(p: o=92: 8)$, $p: o$ ratio determined by ${ }^{1} \mathrm{H}$ NMR. The product was obtained as colourless liquid $(0.175 \mathrm{~g}, 94 \%$ yield $): R_{f}=0.6$ petroleum ether/EtOAc (80:20); IR (neat) $\mathrm{cm}^{-1}:$ 3290, 2896, 2126, 1738, 1606, 1487, 1442, 1301, 1238, 1140, 1031, 1002, 931, 800, 760, 676, 640; ${ }^{1} \mathrm{H}$ NMR (400 MHz, $\left.\mathrm{CDCl}_{3}\right): \delta 7.51-7.39(\mathrm{~m}$, 2H), $7.24-7.13(\mathrm{~m}, 2 \mathrm{H}), 6.82-6.69(\mathrm{~m}, 3 \mathrm{H}), 5.94(\mathrm{~s}, 2 \mathrm{H}), 4.92(\mathrm{~s}, 1 \mathrm{H}), 4.78-4.68(\mathrm{~m}, 2 \mathrm{H})$, $2.48(\mathrm{t}, J=2.5 \mathrm{~Hz}, 1 \mathrm{H}) ;{ }^{13} \mathrm{C} \mathrm{NMR}\left(100 \mathrm{MHz}, \mathrm{CDCl}_{3}\right): \delta 171.4,148.1,147.2,137.4,131.9$, 131.5, 130.3, 122.0, 121.7, 109.1, 108.5, 101.4, 77.3, 75.5, 55.7, 52.9; HRMS (ESI TOF): Calculated for $\mathrm{C}_{18} \mathrm{H}_{13} \mathrm{BrNaO}_{4}(\mathrm{M}+\mathrm{Na})^{+}$: 394.9895, Found: 394.9894 .

\section{Prop-2-yn-1-yl 2-(benzo[d][1,3]dioxol-5-yl)-2-(p-tolyl)acetate (3hk):}<smiles>C#CCOC(=O)C(c1ccc(C)cc1)c1ccc2c(c1)OCO2</smiles>

Compound 3hk was synthesized following the general procedure (D). The product was obtained as a major product para-isomer $(p: o=98: 2)$, $p$ :o ratio determined by ${ }^{1} \mathrm{H}$ NMR. The product was obtained as white solid $(0.094 \mathrm{~g}, 61 \%$ yield $): R_{f}=0.6$ petroleum ether/EtOAc (80:20); m.p.: 59-61 ${ }^{\circ} \mathrm{C}$; IR (neat) $\mathrm{cm}^{-1}:$ 3288, 2894, 2779, 2129, 1739, 1611, 1489, 1441, 1306, 1241, 1185, 1140, 1034, 997, 932, 808, 755, 681; ${ }^{1} \mathrm{H}$ NMR (400 MHz, $\left.\mathrm{CDCl}_{3}\right): \delta 7.23-7.16(\mathrm{~m}, 2 \mathrm{H}), 7.13(\mathrm{~d}, J=8.0 \mathrm{~Hz}, 2 \mathrm{H}), 6.86-6.78(\mathrm{~m}, 1 \mathrm{H}), 6.78-6.69(\mathrm{~m}$, 2H), $5.92(\mathrm{~s}, 2 \mathrm{H}), 4.94(\mathrm{~s}, 1 \mathrm{H}), 4.72(\mathrm{~d}, J=2.5 \mathrm{~Hz}, 2 \mathrm{H}), 2.46$ (t, $J=2.5 \mathrm{~Hz}, 1 \mathrm{H}), 2.32(\mathrm{~s}, 3 \mathrm{H})$; ${ }^{13} \mathrm{C} \mathrm{NMR}\left(100 \mathrm{MHz}, \mathrm{CDCl}_{3}\right): \delta 172.0,148.0,147.0,137.3,135.4,132.3,129.5,128.4,122.0$, 109.2, 108.3, 101.2, 77.5, 75.3, 56.0, 52.7, 21.2; HRMS (ESI TOF): Calculated for $\mathrm{C}_{19} \mathrm{H}_{17} \mathrm{O}_{4}$ $(\mathrm{M}+\mathrm{H})^{+}:$309.1127, Found: 309.1127. 
<smiles>C#CCOC(=O)C(c1ccc(OC)cc1)c1ccc2c(c1)OCO2</smiles>

Compound 3ik was synthesized following the general procedure (D). The product was obtained as a major product para-isomer ( $p: o=99: 1), p: o$ ratio determined by ${ }^{1} \mathrm{H}$ NMR. The product was obtained as colourless liquid $(0.089 \mathrm{~g}, 55 \%$ yield $): R_{f}=0.5$ petroleum ether/EtOAc (80:20); IR (neat) $\mathrm{cm}^{-1}:$ 3287, 2904, 2124, 1740, 1610, 1501, 1443, 1367, 1246, 1143, 1034, 933, 807, 760, 683, 637; ${ }^{1} \mathrm{H}$ NMR (400 MHz, $\left.\mathrm{CDCl}_{3}\right): \delta 7.24-7.19(\mathrm{~m}, 2 \mathrm{H})$, $6.88-6.82(\mathrm{~m}, 2 \mathrm{H}), 6.80(\mathrm{~s}, 1 \mathrm{H}), 6.77-6.70(\mathrm{~m}, 2 \mathrm{H}), 5.91(\mathrm{~s}, 2 \mathrm{H}), 4.93(\mathrm{~s}, 1 \mathrm{H}), 4.72(\mathrm{~d}, J=$ $2.5 \mathrm{~Hz}, 2 \mathrm{H}), 3.77(\mathrm{~s}, 3 \mathrm{H}), 2.47(\mathrm{t}, J=2.5 \mathrm{~Hz}, 1 \mathrm{H}) ;{ }^{13} \mathrm{C} \mathrm{NMR}\left(100 \mathrm{MHz}, \mathrm{CDCl}_{3}\right): \delta 172.1$, 159.0, 148.0, 147.0, 132.5, 130.5, 129.6, 121.9, 114.2, 109.2, 108.3, 101.2, 77.5, 75.3, 55.5, 55.4, 52.7; HRMS (ESI TOF): Calculated for $\mathrm{C}_{19} \mathrm{H}_{17} \mathrm{O}_{5}(\mathrm{M}+\mathrm{H})^{+}$: 325.1076, Found: 325.1092 .

\section{Prop-2-yn-1-yl 2-(4-bromophenyl)-2-(2,5-dimethylfuran-3-yl)acetate (3bl):}<smiles>C#CCOC(=O)C(c1ccc(Br)cc1)c1cc(C)oc1C</smiles>

Compound 3 bl was synthesized following the general procedure (D). The product was obtained as colourless liquid (0.104 g, 60\% yield): $R_{f}=0.5$ petroleum ether/EtOAc (80:20); IR (neat) $\mathrm{cm}^{-1}$ : 3296, 2924, 2126, 1742, 1583, 1487, 1222, 1146, 1079, 1003, 935, 805, 762, 677, 640; ${ }^{1} \mathrm{H}$ NMR (400 MHz, $\left.\mathrm{CDCl}_{3}\right): \delta 7.44$ (d, $\left.J=8.4 \mathrm{~Hz}, 2 \mathrm{H}\right), 7.18$ (d, $\left.J=8.4 \mathrm{~Hz}, 2 \mathrm{H}\right)$, $5.98(\mathrm{~s}, 1 \mathrm{H}), 4.75(\mathrm{~s}, 1 \mathrm{H}), 4.71(\mathrm{dd}, J=2.5,1.9 \mathrm{~Hz}, 2 \mathrm{H}), 2.47(\mathrm{t}, J=2.5 \mathrm{~Hz}, 1 \mathrm{H}), 2.21(\mathrm{~s}$, $3 \mathrm{H}), 2.17(\mathrm{~s}, 3 \mathrm{H}) ;{ }^{13} \mathrm{C} \mathrm{NMR}\left(100 \mathrm{MHz}, \mathrm{CDCl}_{3}\right): \delta 171.4,150.1,146.9,137.3,131.8,129.9$, 121.5, 116.4, 106.8, 77.3, 75.4, 52.8, 47.3, 13.6, 11.8; HRMS (ESI TOF): Calculated for $\mathrm{C}_{17} \mathrm{H}_{16} \mathrm{BrO}_{3}(\mathrm{M}+\mathrm{H})^{+}:$347.0283, Found: 347.0290 . 


\section{General procedure $\mathrm{E}$ for gram-scale synthrsis of $\alpha$-diarylacetates}

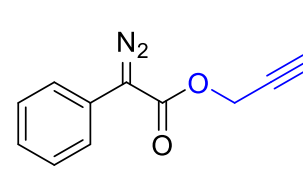

$1 \mathbf{a}$

(1.5 g, 1 equiv.)

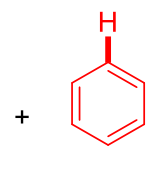

$2 \mathbf{a}$

(10 equiv.)

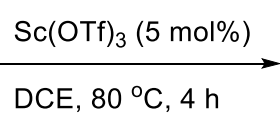

CE, $80^{\circ}, 4 \mathrm{~h}$

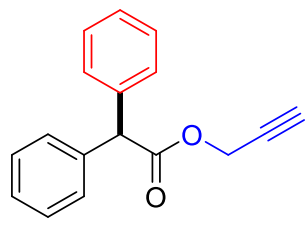

3aa

$(1.31 \mathrm{~g}, 70 \%)$

In a dried $100 \mathrm{~mL}$ RB flask, $\operatorname{Sc}(\mathrm{OTf})_{3}(0.184 \mathrm{~g}, 5 \mathrm{~mol} \%)$, propargyl $\alpha$-phenyl- $\alpha$ diazoacetate 1a (1.5 g, 1 equiv.) and benzene 2a (6.7 mL, 10 equiv.) was added in $25 \mathrm{~mL}$ of DCE at room temperature under inert atmosphere. Then the reaction mixture was refluxed at $80{ }^{\circ} \mathrm{C}$ over $4 \mathrm{~h}$. The progress of the reaction was monitored by TLC. After completion of the reaction, the solvent was removed under reduced pressure. The crude product obtained was purified by column chromatography over silica gel to furnish the desired product $\mathbf{3 a a}$ as white solid (1.31 g, 70\% yield).

\section{Prop-2-yn-1-yl 2,2-diphenylacetate (3aa):}<smiles>C#CCOC(=O)C(c1ccccc1)c1ccccc1</smiles>

Compound 3aa was synthesized following the general procedure (E). The product was obtained as white solid ( $1.31 \mathrm{~g}, 70 \%$ yield): $R_{f}=0.5$ petroleum ether/EtOAc (95:5); m.p.: 46-48 ${ }^{\circ} \mathrm{C}$; IR (neat) $\mathrm{cm}^{-1}: 3289,3031,2941,2126,1739,1598,1494,1448,1370,1220,1181$, 1080, 997, 739, 694, 638, 564; ${ }^{1} \mathrm{H}$ NMR (400 MHz, $\left.\mathrm{CDCl}_{3}\right): \delta 7.49-7.12(\mathrm{~m}, 10 \mathrm{H}), 5.07$ (s, $1 \mathrm{H}), 4.74(\mathrm{~d}, J=2.5 \mathrm{~Hz}, 2 \mathrm{H}), 2.46(\mathrm{t}, J=2.5 \mathrm{~Hz}, 1 \mathrm{H}) ;{ }^{13} \mathrm{C} \mathrm{NMR}\left(100 \mathrm{MHz}, \mathrm{CDCl}_{3}\right): \delta 171.8$, 138.3, 128.8, 128.7, 127.5, 77.4, 75.3, 56.8, 52.7; HRMS (ESI TOF): Calculated for $\mathrm{C}_{17} \mathrm{H}_{14} \mathrm{NaO}_{2}(\mathrm{M}+\mathrm{Na})^{+}: 273.0891$, Found: 273.0891 . 
Prop-2-yn-1-yl 2-(4-chlorophenyl)-2-phenylacetate (3ca):<smiles>C#CCOC(=O)C(c1ccccc1)c1ccc(Cl)cc1</smiles>

Compound 3ca was synthesized following the general procedure (E). The product was obtained as white solid ( $1.31 \mathrm{~g}, 72 \%$ yield): $R_{f}=0.4$ petroleum ether/EtOAc (95:5); m.p.: 83$85{ }^{\circ} \mathrm{C}$; IR (neat) $\mathrm{cm}^{-1}: 3293,3033,2127,1739,1598,1490,1272,1222,1138,1001,813$, 751, 691, 636, 550; ${ }^{1} \mathrm{H}$ NMR (400 MHz, $\left.\mathrm{CDCl}_{3}\right): \delta 7.35-7.24(\mathrm{~m}, 9 \mathrm{H}), 5.03(\mathrm{~s}, 1 \mathrm{H}), 4.79-$ 4.69 (m, 2H), 2.47 (t, $J=2.5 \mathrm{~Hz}, 1 \mathrm{H}) ;{ }^{13} \mathrm{C}$ NMR (100 MHz, DMSO-d 6 ): $\delta 171.0,138.4$, 137.7, 132.0, 130.4, 128.7, 128.5, 128.3, 127.3, 78.1 (for 2-C), 54.5, 52.6; HRMS (ESI TOF): Calculated for $\mathrm{C}_{17} \mathrm{H}_{13} \mathrm{ClNaO}_{2}(\mathrm{M}+\mathrm{Na})^{+}$: 307.0502, Found: 307.0505 .

\section{Prop-2-yn-1-yl 2-(3,4-dichlorophenyl)-2-phenylacetate (3ea):}<smiles>C#CCOC(=O)C(c1ccccc1)c1ccc(Cl)c(Cl)c1</smiles>

Compound 3ea was synthesized following the general procedure (E). The product was obtained as colourless liquid ( $1.46 \mathrm{~g}, 82 \%$ yield): $R_{f}=0.35$ petroleum ether/EtOAc (95:5); IR (neat) $\mathrm{cm}^{-1}: 3295,3071,2948,2124,1743,1596,1470,1269,1145,1028,907,772,702 ;{ }^{1} \mathrm{H}$ NMR (400 MHz, $\left.\mathrm{CDCl}_{3}\right): \delta 7.42(\mathrm{~d}, J=2.2 \mathrm{~Hz}, 1 \mathrm{H}), 7.41-7.26(\mathrm{~m}, 6 \mathrm{H}), 7.16(\mathrm{dd}, J=8.3$, $2.2 \mathrm{~Hz}, 1 \mathrm{H}), 5.00(\mathrm{~s}, 1 \mathrm{H}), 4.80-4.69(\mathrm{~m}, 2 \mathrm{H}), 2.49$ (t, $J=2.5 \mathrm{~Hz}, 1 \mathrm{H}) ;{ }^{13} \mathrm{C} \mathrm{NMR}(100 \mathrm{MHz}$, $\left.\mathrm{CDCl}_{3}\right): \quad \delta 171.0,138.4,137.2,132.8,131.8,130.7,130.6,129.1,128.5,128.2,128.0$, 75.6(for 2-C), 55.8, 53.0; HRMS (ESI TOF): Calculated for $\mathrm{C}_{17} \mathrm{H}_{12} \mathrm{Cl}_{2} \mathrm{NaO}_{2}(\mathrm{M}+\mathrm{Na})^{+}$: 341.0112, Found: 341.0099. 
<smiles>C#CCOC(=O)C(c1ccc(Br)cc1)c1cc(OC)ccc1OC</smiles>

Compound 3bi was synthesized following the general procedure (E). The product was obtained as white solid (1.84 g, 88\% yield): $R_{f}=0.45$ petroleum ether/EtOAc (80:20); m.p.: 97-99 ${ }^{\circ} \mathrm{C}$; IR (neat) $\mathrm{cm}^{-1}: 3289,2944,2837,2126,1741,1593,1494,1222,1148,1010,945$, 806, 712, 641, 537; ${ }^{1} \mathrm{H}$ NMR (400 MHz, $\left.\mathrm{CDCl}_{3}\right): \delta 7.49-7.42(\mathrm{~m}, 2 \mathrm{H}), 7.23-7.17(\mathrm{~m}, 2 \mathrm{H})$, $6.86-6.73(\mathrm{~m}, 2 \mathrm{H}), 6.62(\mathrm{~d}, J=2.8 \mathrm{~Hz}, 1 \mathrm{H}), 5.25(\mathrm{~s}, 1 \mathrm{H}), 4.78-4.67(\mathrm{~m}, 2 \mathrm{H}), 3.77(\mathrm{~s}, 3 \mathrm{H})$, $3.70(\mathrm{~s}, 3 \mathrm{H}), 2.45$ (t, $J=2.5 \mathrm{~Hz}, 1 \mathrm{H}) ;{ }^{13} \mathrm{C} \mathrm{NMR}\left(100 \mathrm{MHz}, \mathrm{CDCl}_{3}\right): \delta$ 171.7, 153.6, 151.1, $136.3,131.9,130.9,128.0,121.6,115.9,112.7,111.5,77.7,75.1,56.2,55.8,52.6,50.4$; HRMS (ESI TOF): Calculated for $\mathrm{C}_{19} \mathrm{H}_{17} \mathrm{BrO}_{4}(\mathrm{M})^{+}: 388.0310$, Found: 388.0305 .

\section{General procedure $\mathbf{F}$ for the deprotection of propargyl group}<smiles>C#CCOC(=O)C(c1ccccc1)c1ccccc1</smiles>

(1.25 g, 1 equiv.)

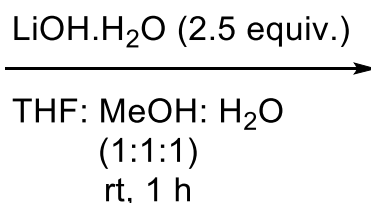

rt, $1 \mathrm{~h}$<smiles>O=C(O)C(c1ccccc1)c1ccccc1</smiles>

$(95 \%)$

A mixture of diarylacetate 3aa (1.25 g, 1 equiv.) and $\mathrm{LiOH}_{\mathrm{H}} \mathrm{H}_{2} \mathrm{O}(0.524 \mathrm{~g}, 2.5$ equiv.) was taken in $100 \mathrm{~mL}$ RB flask \& $30 \mathrm{~mL}$ solvent (THF: $\mathrm{MeOH}: \mathrm{H}_{2} \mathrm{O}=1: 1: 1$ ) was added in above mixture under open atmosphere. The reaction mixture was stirred at room temperature over $1 \mathrm{~h}$. The progress of the reaction was monitored by TLC. After completion of the reaction, the reaction mixture was acidified by using $3 \mathrm{~N} \mathrm{HCl}$ and adjusted to $\mathrm{pH} 2-3$. The crude product was extracted by using ethyl acetate. Solvent was removed under reduced pressure $\&$ crude product was purified by column chromatography over silica gel (100-200 mesh size) to furnish the product $4 \mathbf{a a}$ as a white solid (1.01 g, 95\% yield). 


\section{2,2-Diphenylacetic acid (4aa):}<smiles>O=C(O)C(c1ccccc1)c1ccccc1</smiles>

Compound 4aa was synthesized following the general procedure (F). The product was obtained as white solid (1.01 g, 95\% yield). $R_{f}=0.5$ petroleum ether/EtOAc (70:30); m.p.: 147-149 ${ }^{\circ} \mathrm{C} ;{ }^{1} \mathrm{H}$ NMR $\left(400 \mathrm{MHz}, \mathrm{CDCl}_{3}\right): \delta 7.45-7.16(\mathrm{~m}, 10 \mathrm{H}), 5.04(\mathrm{~s}, 1 \mathrm{H}) ;{ }^{13} \mathrm{C} \mathrm{NMR}$ (100 MHz, $\mathrm{CDCl}_{3}$ ): $\delta 178.9,138.0,128.8$ (for two carbon), 127.6, 57.1; IR (neat) $\mathrm{cm}^{-1}: 3027$, 2910, 1699, 1494, 1451, 1411, 1306, 1218, 1031, 934, 737, 698, 635; HRMS (ESI TOF): Calculated for $\mathrm{C}_{14} \mathrm{H}_{13} \mathrm{O}_{2}(\mathrm{M}+\mathrm{H})^{+}:$213.0916, Found: 213.0917 .

\section{2-(4-Chlorophenyl)-2-phenylacetic acid (4ca):}<smiles>O=C(O)C(c1ccccc1)c1ccc(Cl)cc1</smiles>

Compound 4ca was synthesized following the general procedure $(\mathrm{F})$. The product was obtained as white solid (1.02 g, 94\% yield). $R_{f}=0.25$ petroleum ether/EtOAc (80:20); m.p.: 117-119 ${ }^{\circ} \mathrm{C}$; IR (neat) $\mathrm{cm}^{-1}: 3029,2695,1704,1599,1491,1452,1408,1280,1213,1091$, 1015, 930, 812, 749, 700, 663; ${ }^{1} \mathrm{H}$ NMR ((400 MHz, $\left.\mathrm{CDCl}_{3}\right): \delta 7.39-7.20(\mathrm{~m}, 9 \mathrm{H}), 5.01(\mathrm{~s}$, $1 \mathrm{H}) ;{ }^{13} \mathrm{C} \mathrm{NMR}\left(100 \mathrm{MHz}, \mathrm{CDCl}_{3}\right): \delta 178.6,137.5,136.4,133.7,130.2,129.0$ (for 2 Carbons), 128.7, 127.9, 56.4; HRMS (ESI TOF): Calculated for $\mathrm{C}_{14} \mathrm{H}_{11} \mathrm{ClNaO}_{2}(\mathrm{M}+\mathrm{Na})^{+}$: 269.0345, Found: 269.0342.

\section{2-(3,4-Dichlorophenyl)-2-phenylacetic acid (4ea):}<smiles>O=C(O)C(c1ccccc1)c1ccc(Cl)c(Cl)c1</smiles> 
Compound 4ea was synthesized following the general procedure $(\mathrm{F})$. The product was obtained as white solid (1.06 g, 96\% yield). $R_{f}=0.30$ petroleum ether/EtOAc (75:25); m.p.: $118-121^{\circ} \mathrm{C}$; IR (neat) $\mathrm{cm}^{-1}: 3028,1706,1596,1560,1469,1404,1274,1214,1136,1032$, 917, 816, 755, 705; ${ }^{1} \mathrm{H}$ NMR (400 MHz, $\left.\mathrm{CDCl}_{3}\right): \delta 7.45-7.29(\mathrm{~m}, 7 \mathrm{H}), 7.18(\mathrm{dd}, J=8.4,2.1$ $\mathrm{Hz}, 1 \mathrm{H}), 5.00(\mathrm{~s}, 1 \mathrm{H}) ;{ }^{13} \mathrm{C} \mathrm{NMR}\left(100 \mathrm{MHz}, \mathrm{CDCl}_{3}\right): \delta 178.0,138.0,136.9,132.9,132.0$, 130.8, 130.7, 129.1, 128.6, 128.2, 128.2, 56.1. HRMS (ESI TOF): Calculated for $\mathrm{C}_{14} \mathrm{H}_{11} \mathrm{Cl}_{2} \mathrm{O}_{2}(\mathrm{M}+\mathrm{Na})^{+}:$302.9956, Found: 302.9948 .

\section{Procedure for gram-scale synthesis of Adiphenine: antispasmodic drug}

A mixture of 2,2-diphenylacetic acid 4aa ( 0.9 g, 1 equiv.), sodium carbonate ( $2.25 \mathrm{~g}$, 5 equiv.) and diethyl amine ( $8.8 \mathrm{~mL}, 20$ equiv.) was stirred in $30 \mathrm{~mL}$ of DCE at room temperature in $100 \mathrm{~mL} \mathrm{RB}$ flask under inert atmosphere for 5 minutes. The reaction mixture was refluxed at $80{ }^{\circ} \mathrm{C}$ over $16 \mathrm{~h}$. The progress of the reaction was monitored by TLC. After completion of the reaction, the solvent was removed under reduced pressure. The residue was diluted with water $(30 \mathrm{~mL})$ and extracted with ethyl acetate $(3 \times 30 \mathrm{~mL})$. The combined organic layers were washed with cold water and brine and dried over $\mathrm{Na}_{2} \mathrm{SO}_{4}$, filtered and the filtrate was evaporated under vacuum. The crude product obtained was purified by column chromatography over silica gel to furnish the desired product $\mathbf{2 1}$ obtained as colourless liquid (1.12 g, 85\% yield).

\section{2-(Diethylamino)ethyl 2,2-diphenylacetate (21):}

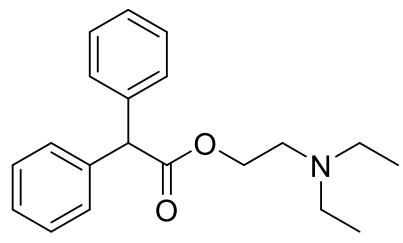

The compound 21 was obtained as colourless liquid (1.12 g, 85\% yield). $R_{f}=0.30$ petroleum ether/EtOAc (75:25); IR (neat) cm $\mathrm{cm}^{-1}$ : 2969, 2805, 1735, 1597, 1496 1447, 1304, 1189, 1149, 1079, 1020, 741, 697. ${ }^{1} \mathrm{H}$ NMR (400 MHz, $\left.\mathrm{CDCl}_{3}\right): \delta 7.38-7.20(\mathrm{~m}, 10 \mathrm{H}), 5.03$ $(\mathrm{s}, 1 \mathrm{H}), 4.22(\mathrm{t}, J=6.2 \mathrm{~Hz}, 2 \mathrm{H}), 2.68(\mathrm{t}, J=6.2 \mathrm{~Hz}, 2 \mathrm{H}), 2.50(\mathrm{q}, J=7.2 \mathrm{~Hz}, 4 \mathrm{H}), 0.97$ (t, $J=$ $7.2 \mathrm{~Hz}, 4 \mathrm{H}) ;{ }^{13} \mathrm{C}$ NMR $\left(100 \mathrm{MHz}, \mathrm{CDCl}_{3}\right): \delta 172.6,138.8,128.8,128.7,127.3,63.6,57.3$, 51.1, 47.7, 12.0; HRMS (ESI TOF): Calculated for $\mathrm{C}_{20} \mathrm{H}_{26} \mathrm{NO}_{2}(\mathrm{M}+\mathrm{H})^{+}:$312.1964, Found: 312.1969 . 


\section{Mechanistic investigations:}

\section{Appendix-III: Studies of Kinetic isotopic effect:}

A)<smiles>C#CCOC(=O)C(=N)c1ccc(Br)cc1</smiles>

1b<smiles>c1ccccc1</smiles>

2a<smiles>[2H]c1c([2H])c2c([2H])c(c1[2H])OCCC2</smiles>

2a-d

(0.5 mmol, 1 equiv.) (2.5 mmol, 5 equiv.) ( $2.5 \mathrm{mmol}, 5$ equiv.)

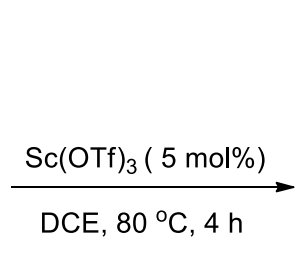<smiles>C#CCOC(=O)C(c1ccc(Br)cc1)c1c(O)c([18OH])c([18OH])c([18OH])c1[18OH]</smiles>
$\mathrm{H}: \mathrm{D}=50: 50$ $K_{\mathrm{H}} / K_{\mathrm{D}}=1$ Cleavage of $\mathrm{C}-\mathrm{H}$ bond is not R.D.S

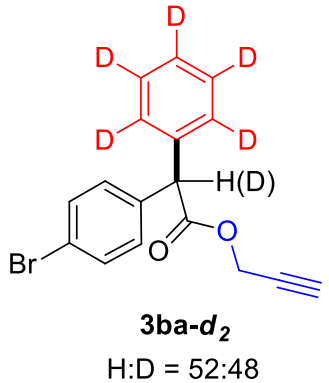

Concerted as well as stepwise

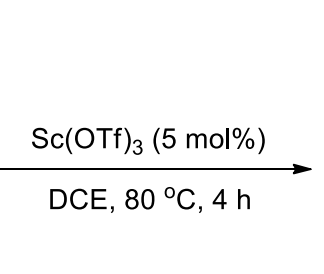

B)<smiles>C#CCOC(=O)C(=N)c1ccc(Br)cc1</smiles>

(0.5 mmol, 1 equiv.)<smiles>[2H]c1c([2H])c([2H])c([2H])c([2H])c1[2H]</smiles>

2a-d

(5 mmol, 10 equiv.)

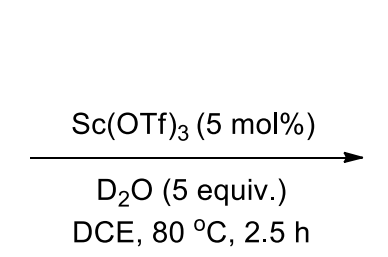

DCE, $80^{\circ} \mathrm{C}, 2.5 \mathrm{~h}$

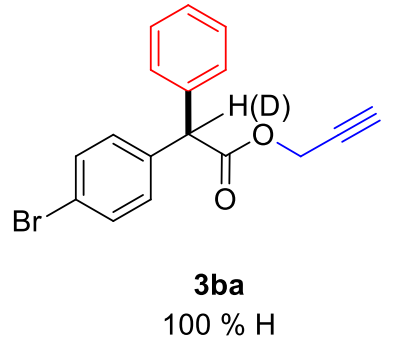

$100 \% \mathrm{H}$

No H-D exchange after product formation

Reaction-A: In a dried $25 \mathrm{~mL}$ RB flask, $\mathrm{Sc}(\mathrm{OTf})_{3}(12.3 \mathrm{mg}, 5 \mathrm{~mol} \%)$, propargyl $\alpha-(4-$ bromophenyl)- $\alpha$-diazoacetate $\mathbf{1 b}(0.140 \mathrm{~g}, 1$ equiv., $0.5 \mathrm{mmol})$, benzene $2 \mathbf{a}(0.223 \mathrm{~mL}, 5$ equiv., $2.5 \mathrm{mmol})$ and benzene- $\mathrm{d}_{6} \mathbf{2 a - d}(0.222 \mathrm{~mL}, 5$ equiv., $2.5 \mathrm{mmol})$ were added in 2.5 $\mathrm{mL}$ of DCE at room temperature under inert atmosphere. Then the reaction mixture was refluxed at $80{ }^{\circ} \mathrm{C}$ over $4 \mathrm{~h}$. The progress of the reaction was monitored by TLC. After completion of the reaction, the solvent was removed under reduced pressure. The crude product obtained was purified by column chromatography over silica gel to furnish the desired product $\mathbf{3 b a}-\boldsymbol{d}_{1}$. 
Reaction-B: In a dried $25 \mathrm{~mL} \mathrm{RB}$ flask, $\mathrm{Sc}(\mathrm{OTf})_{3}(12.3 \mathrm{mg}, 5 \mathrm{~mol} \%)$, propargyl $\alpha$-(4bromophenyl)- $\alpha$-diazoacetate $\mathbf{1 b}(0.140$ g, 1 equiv., $0.5 \mathrm{mmol})$ and benzene- $\mathrm{d}_{6} \mathbf{2 a - d}(0.443$ $\mathrm{mL}, 10$ equiv., $5 \mathrm{mmol}$ ) were added in $2.5 \mathrm{~mL}$ of DCE at room temperature under inert atmosphere. Then the reaction mixture was refluxed at $80{ }^{\circ} \mathrm{C}$ over $4 \mathrm{~h}$. The progress of the reaction was monitored by TLC. After completion of the reaction, the solvent was removed under reduced pressure. The crude product obtained was purified by column chromatography over silica gel to furnish the desired product $\mathbf{3 b a}-\boldsymbol{d}_{2}$.

Reaction-C: In a dried 25 mL RB flask, Sc(OTf) 3 (12.3 mg, 5 mol\%), Prop-2-yn-1-yl 2-(4bromophenyl)-2-phenylacetate $3 \mathbf{b a}(0.165 \mathrm{~g}, 1$ equiv., $0.5 \mathrm{mmol})$ and deuterium oxide $(0.045$ $\mathrm{mL}, 5$ equiv., $2.5 \mathrm{mmol}$ ) were added in $2.5 \mathrm{~mL}$ of DCE at room temperature under inert atmosphere. Then the reaction mixture was refluxed at $80{ }^{\circ} \mathrm{C}$ over $2.5 \mathrm{~h}$. The progress of the reaction was monitored by TLC. After completion of the reaction, the solvent was removed under reduced pressure. The crude product obtained was purified by column chromatography over silica gel. The product $\mathbf{3 b a}$ was obtained as one and only product.

\section{Appendix-IV: Computational Studies:}

The calculations have been carried out with Turbomole 7.2 [1,2] using the TZVP basis set [3]. Geometry optimizations were performed using the Perdew, Burke, and Ernzerhof functional (PBE) [4]. Dispersion corrections [5] have been included in all the calculations. The resolution of identity (RI) [6] along with the multipole accelerated RI (marij) [7] approximations have been used for an accurate and efficient treatment of the electronic Coulomb term in the DFT calculations. Solvent corrections have also been included in all the calculations using the cosmo model [8], with epsilon $(\varepsilon)$ $=10.4$, employed to model the solvent dichloroethane, which has been employed experimentally to study the reaction involved. Local reactivity is described in terms of the Fukui function, which represents the change of the electronic density in a given point of the molecule with respect to the change in the number of electrons. The Fukui function is usually the correct reactivity indicator for predicting the reaction mechanism. [9-11] These descriptors are useful to evaluate the relevant sites in a molecule where nucleophilic or electrophilic attack can take place[12]. The efficiency of the catalytic cycle for the reaction mechanism investigated has been calculated with the AUTOF [13,14] program by employing the "Energetic Span Model" (ESM), developed by Shaik and co-workers [1517]. This has been done for all the free energy profiles discussed in the manuscript. The turnover frequency (TOF) calculations take into account the principal rate-determining transition state, as well 
as the potentially rate-influencing transition states and intermediates during the catalysis process. In most cases, the TOF is calculated from the TOF-determining transition state (TDTS), the TOFdetermining intermediate (TDI), and from the reaction energy, $\Delta G_{\mathrm{r}}$ as shown below:

$\mathrm{TOF}=\frac{\mathrm{KbT}}{\mathrm{h}} \mathrm{e}^{-\delta \mathrm{E} / \mathrm{RT}}$

$\delta \mathrm{E}=\mathrm{TDTS}-\mathrm{TDI} \quad$ if TDTS appears after TDI

$\delta \mathrm{E}=\mathrm{TDTS}-\mathrm{TDI}-\Delta \mathrm{Gr} \quad$ if TDTS appears before TDI

This model has been employed to calculate the TOFs for the free energy profiles obtained for the mechanisms in the solvent phase discussed in the manuscript. This model can also be employed for stoichiometric reactions, where the TOF would correspond to the efficiency of the reaction.

\section{Results and Discussion (computational approach)}

At first, we looked into the electrophilicity of the carbon center with the diazo functional group, by employing the Fukui function indices. The highest electrophilicity at the incoming carbene carbon was seen for the case when the ester carbon had a propargyl group attached to it, in comparison to other groups (see Table 1).

Table 1. Fukui function indices at the carbene carbon for different reactant complexes.

\begin{tabular}{|c|l|l|l|c|}
\hline Reactant complex & $\begin{array}{l}\text { Hirshfield } \\
\text { population for } \\
\text { Neutral } \\
\text { Species at } \\
\text { Carbene } \\
\text { Carbon }\end{array}$ & $\begin{array}{l}\text { Hirshfield } \\
\text { population for } \\
\text { cationic } \\
\text { species at } \\
\text { Carbene } \\
\text { Carbon }\end{array}$ & $\begin{array}{l}\text { Hirshfield } \\
\text { population for } \\
\text { anionic species } \\
\text { at Carbene } \\
\text { Carbon }\end{array}$ & $\begin{array}{l}\text { Electrophilicity }\left(\mathrm{F}^{+}\right) \\
\text {at Carbene Carbon }\end{array}$ \\
\hline $\begin{array}{c}\text { Propargyl } \alpha \text {-Aryl- } \\
\alpha \text {-diazoacetate }\end{array}$ & -0.001580 & 0.091116 & -0.066365 & 0.064785 \\
\hline $\begin{array}{c}\text { 2,2,2-trifuloro } \\
\text { ethyl } \alpha \text {-Aryl- } \alpha- \\
\text { diazoacetate }\end{array}$ & 0.000779 & 0.088683 & -0.062377 & 0.063156 \\
\hline
\end{tabular}




\begin{tabular}{|c|c|c|c|c|}
\hline $\begin{array}{c}\text { Allyl } \alpha \text {-Aryl- } \alpha- \\
\text { diazoacetate }\end{array}$ & -0.003877 & 0.092164 & -0.066856 & 0.062979 \\
\hline $\begin{array}{c}\text { Ethyl } \alpha \text {-Aryl- } \alpha- \\
\text { diazoacetate }\end{array}$ & -0.003250 & 0.092229 & -0.064495 & 0.061245 \\
\hline $\begin{array}{c}\text { Propargyl } \alpha \text {-Aryl- } \\
\alpha \text {-diazoacetate- } \\
\text { Sc(OTf) })_{3} \text { complex }\end{array}$ & 0.013038 & 0.069141 & -0.025331 & 0.038369 \\
\hline $\begin{array}{c}2,2,2-\text { trifuloro } \\
\text { ethyl } \alpha \text {-Aryl- } \alpha- \\
\text { diazoacetate- } \\
\text { Sc(OTf) })_{3} \text { complex }\end{array}$ & 0.015817 & 0.070922 & -0.020496 & 0.036313 \\
\hline $\begin{array}{c}\text { Allyl } \alpha \text {-Aryl- } \alpha- \\
\text { diazoacetate- } \\
\text { Sc(OTf })_{3} \text { complex }\end{array}$ & 0.011210 & 0.069464 & -0.02327 & 0.03448 \\
\hline $\begin{array}{c}\text { Sc(OTf) })_{3} \text { complex } \alpha \text {-Aryl- } \alpha- \\
\text { diazoacetate- }\end{array}$ & 0.011551 & 0.076957 & -0.021929 & \\
\hline & & & & \\
\hline
\end{tabular}

To look into the reaction mechanism, we first optimized the propargyl $\alpha$-aryl- $\alpha$ diazoacetate with $\mathrm{Sc}(\mathrm{OTf})_{3}$ to investigate the most stable conformation in which $\mathrm{Sc}(\mathrm{OTf})_{3}$ would interact with the different oxygens of the propargyl $\alpha$-aryl- $\alpha$-diazoacetate (see Figure 1). It was found that $\operatorname{Sc}(\mathrm{OTf})_{3}$ interacts with the carbonyl oxygen in an anti fashion to the diazo group with an interaction energy (Int1) of $-10.0 \mathrm{kcal} / \mathrm{mol}$ (see Figure 1). 


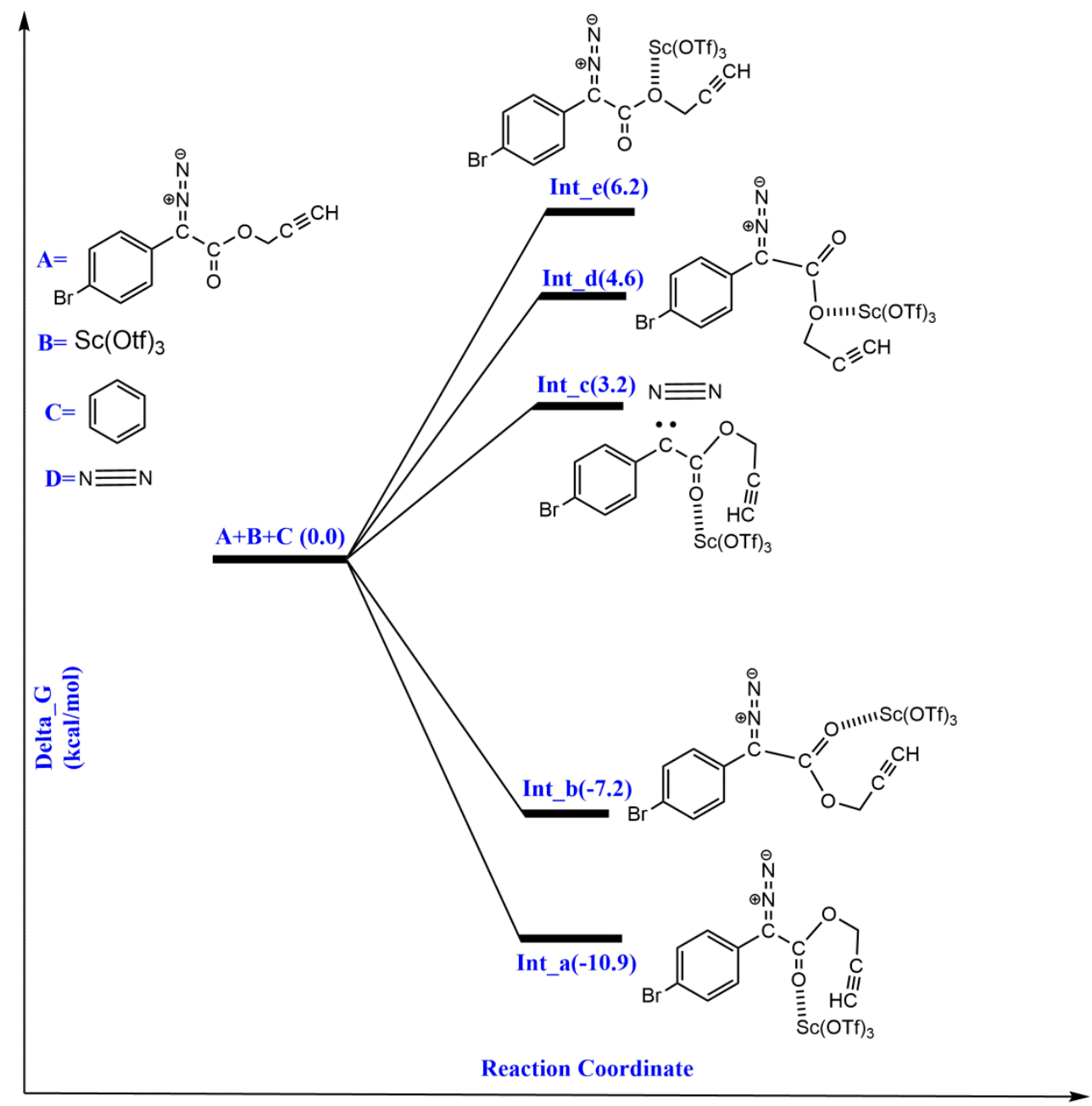

Figure 1. The energy profile for different conformations of interactions of propargyl $\alpha-(4-$ bromophenyl)- $\alpha$-diazoacetate with scandium triflate $\left(\operatorname{Sc}(\mathrm{OTf})_{3}\right)$ catalyst. Energies are in $\mathrm{kcal} / \mathrm{mol}$. 

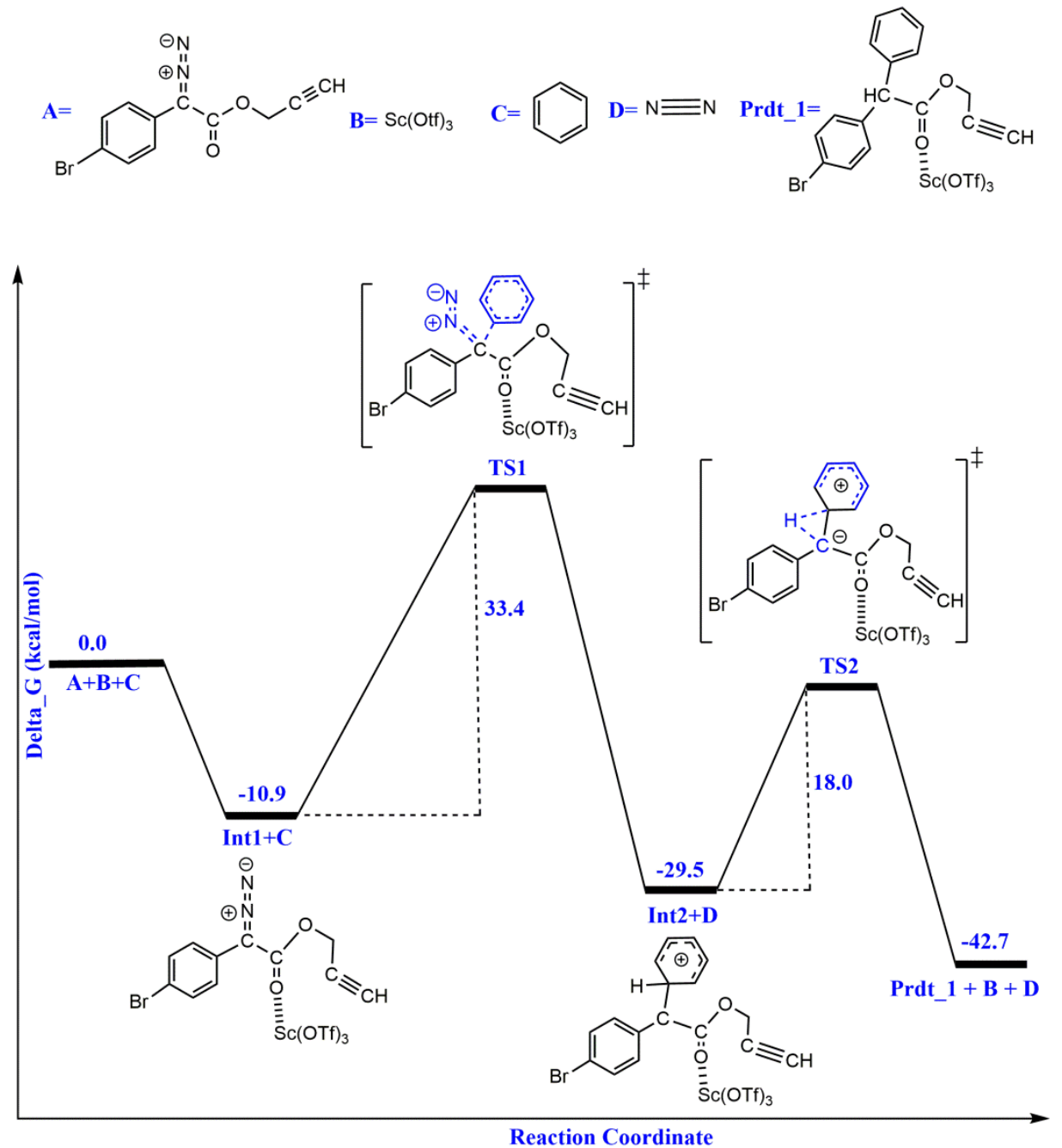

Figure 2. The energy profile for the reaction of propargyl $\alpha$-(4-bromophenyl)- $\alpha$-diazoacetate with benzene, with the scandium triflate $\left(\mathrm{Sc}(\mathrm{OTf})_{3}\right)$ catalyst. Energies are in $\mathrm{kcal} / \mathrm{mol}$.

After the $\operatorname{Sc}(\mathrm{OTf})_{3}$ interacts with the carbonyl oxygen of the propargyl $\alpha$-aryl- $\alpha$ diazoacetate, the arene approaches and interacts with the carbene carbon of the propargyl $\alpha$ aryl- $\alpha$-diazoacetate, removing the diazo as dinitrogen, with a free energy (TS1) of 33.4 $\mathrm{kcal} / \mathrm{mol}$ (see Figure 2). The free energy for the transition state is acceptable for the experimental conditions employed (temperature of $80^{\circ} \mathrm{C}$ ). The arene forms a complex (Int2) with an interaction energy of $-21.5 \mathrm{kcal} / \mathrm{mol}$. At this point, the arene aromatizes (TS2) with a free energy of $18.0 \mathrm{kcal} / \mathrm{mol}$ to form the product (see Figure 2). The product is stable by 42.7 $\mathrm{kcal} / \mathrm{mol}$ (see Figure 2). Thermodynamics and kinetics calculations have also been calculated for the other groups that have been experimentally studied with the $\mathrm{Sc}(\mathrm{OTf})_{3}$ catalyst (see Figure 3-5). The turnover frequency (TOF) has also been calculated and compared for all the energy profiles associated with the different groups (see Table 2). 


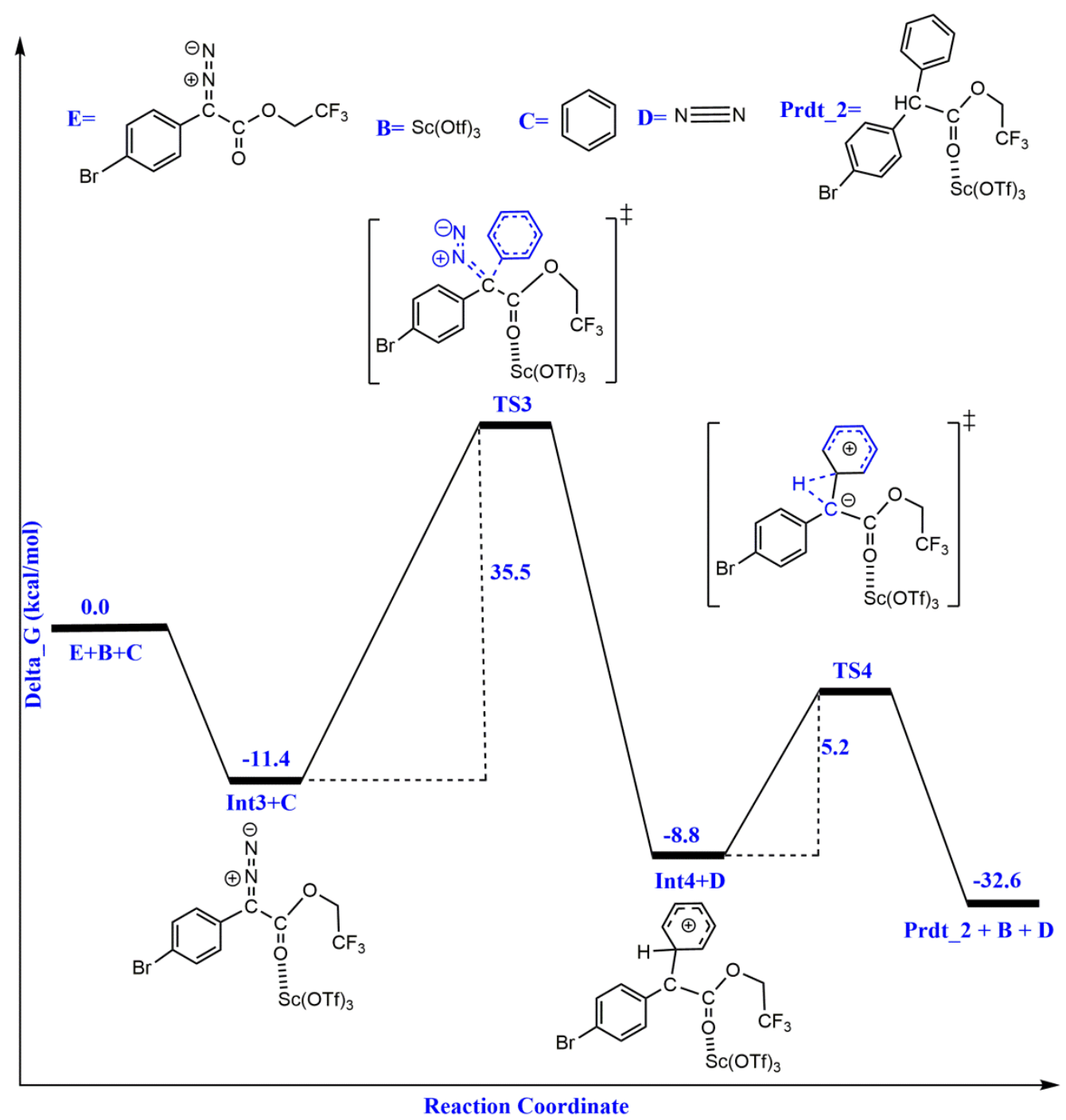

Figure 3. The energy profile for the reaction of 2,2,2-Trifluoroethyl $\alpha$-(4-bromophenyl)- $\alpha$ diazoacetate with benzene, with the scandium triflate $\left[\mathrm{Sc}(\mathrm{OTf})_{3}\right]$ catalyst. Energies are in $\mathrm{kcal} / \mathrm{mol}$. 


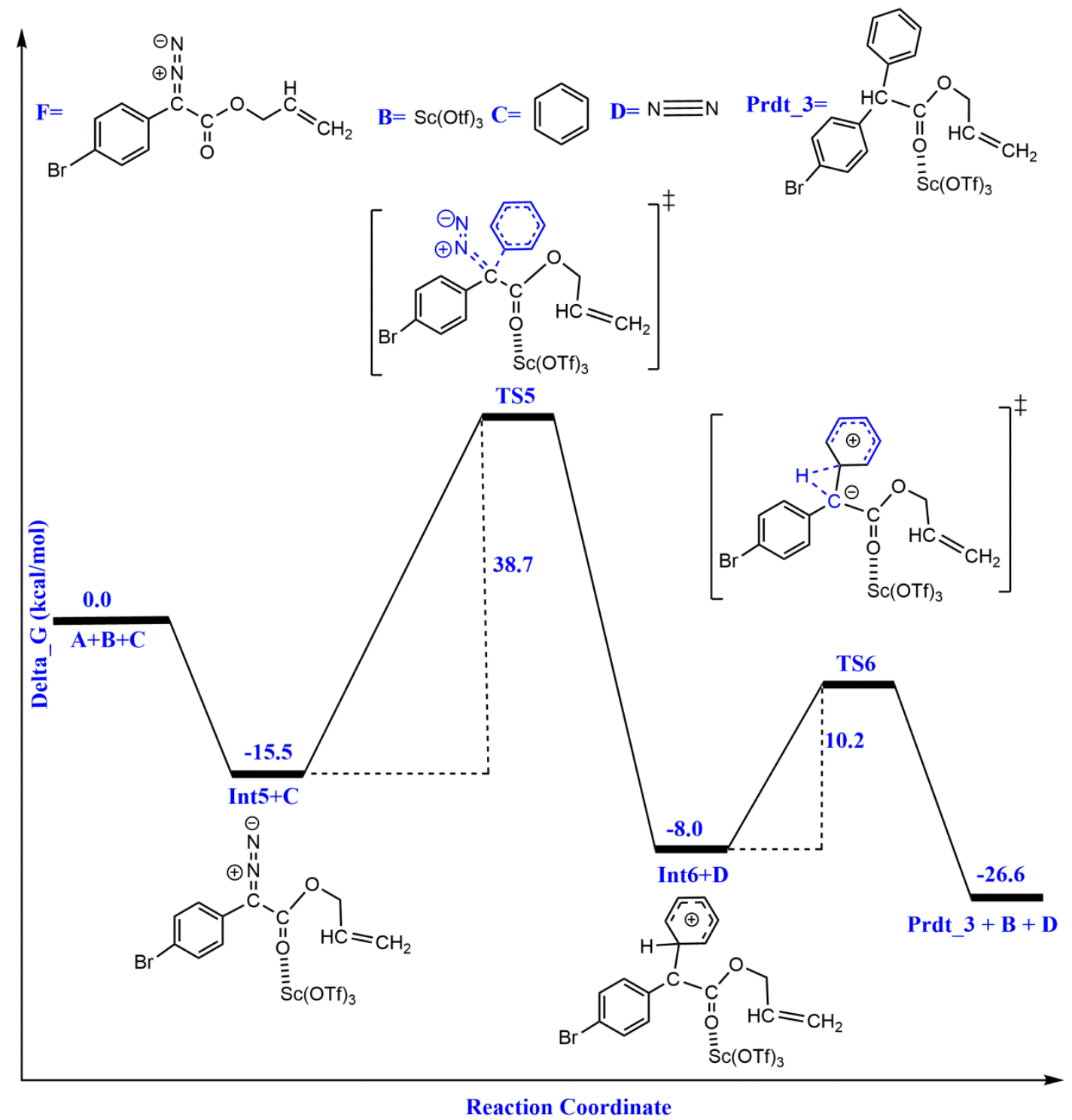

Figure 4. The energy profile for the reaction of allyl $\alpha$-(4-bromophenyl)- $\alpha$-diazoacetate with benzene, with the scandium triflate $\left(\mathrm{Sc}(\mathrm{OTf})_{3}\right)$ catalyst. Energies are in $\mathrm{kcal} / \mathrm{mol}$. 


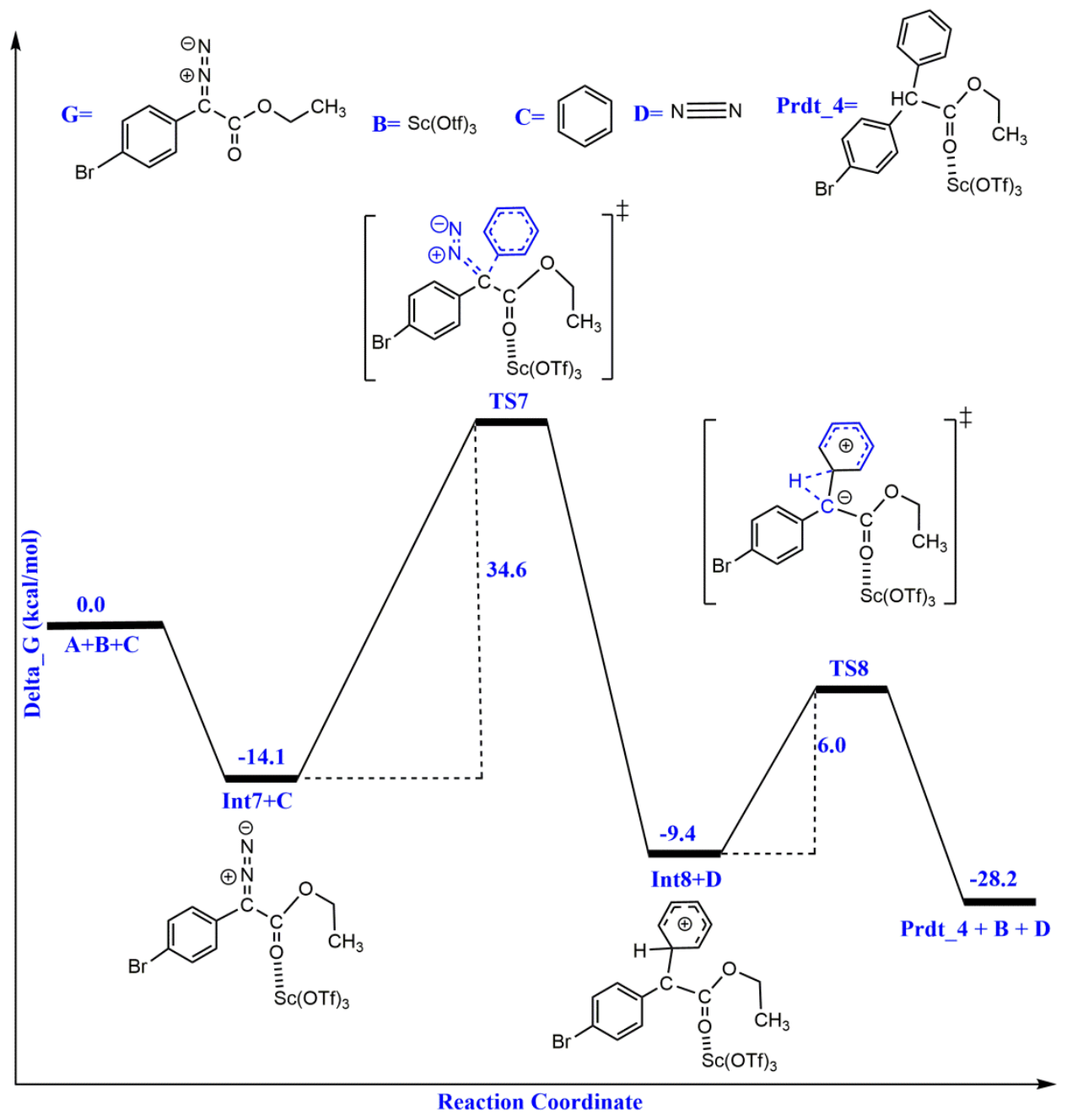

Figure 5. The energy profile for the reaction of ethyl $\alpha$-(4-bromophenyl)- $\alpha$-diazoacetate with benzene, with the scandium triflate $\left(\mathrm{Sc}(\mathrm{OTf})_{3}\right)$ catalyst. Energies are in $\mathrm{kcal} / \mathrm{mol}$.

Table 2. TOFs obtained for the different reactions with the $\mathrm{Sc}(\mathrm{OTf})_{3}$ catalyst.

\begin{tabular}{|c|c|}
\hline Reaction & TOF $\left(\mathrm{h}^{-1}\right)$ \\
\hline Propargyl $\alpha$-Aryl- $\alpha$-diazoacetate $+\operatorname{Sc}(\mathrm{OTf})_{3}$ & $8.6^{*} 10^{-12}$ \\
\hline $2,2,2$-trifuloroethyl $\alpha$-Aryl- $\alpha$-diazoacetate $+\operatorname{Sc}(\mathrm{OTf})_{3}$ & $2.4^{*} 10^{-13}$ \\
\hline Allyl $\alpha$-Aryl- $\alpha$-diazoacetate $+\operatorname{Sc}(\mathrm{OTf})_{3}$ & $7.4 * 10^{-15}$ \\
\hline Ethyl $\alpha$-Aryl- $\alpha$-diazoacetate $+\operatorname{Sc}(\mathrm{OTf})_{3}$ & $1.9 * 10^{-16}$ \\
\hline
\end{tabular}


The TOF calculations suggest that the reaction of propargyl $\alpha$-aryl- $\alpha$-diazoacetates with $\mathrm{Sc}(\mathrm{OTf})_{3}$ will give the product with highest efficiency. This shows that there is a definite role of the propargyl group in activating the arene efficiently.

\section{References:}

[1] Z. Liang, L. Zhang, L. Li, J. Liu, H. Li, L. Zhang, L. Chen, K. Cheng, M. Zheng, X. Wen, P. Zhang, J. Hao, Y. Gong, X. Zhang, X. Zhu, J. Chen, H. Liu, H. Jiang, C. Luo, H. Sun, Eur. J. Med. Chem. 2011, 46, 2011.

[2] S. I. Lee, G.-S. Hwang, D. H. Ryu, J. Am. Chem. Soc. 2013, 135, 7126.

[3] R. Ahlrichs, M. Bär, M. Häser, H. Horn, C. Kölmel, Chem. Phys. Lett. 1989, 162, 165.

[4] Turbomole V6.3 2011, "A Development of University of Karlsruhe and Forschungszentrum Karlsruhe GmbH, 1989-2007, Turbomole GmbH,” 2007. http://www.turbomole.com.

[5] S. Ansgar, H. Christian, A. Reinhart, J. Chem. Phys. 1994, 100, 5829.

[6] J. P. Perdew, K. Burke, M. Ernzerhof Phys. Rev. Lett. 1996, 77, 3865-3868.

[7] S. Grimme, J. Antony, S. Ehrlich, H. Krieg, J. Chem. Phys. 2010, 132, 154104.

[8] K. Eichkorn, O. Treutler, H. Öhm, M. Häser, R. Ahlrichs, Chem. Phys. Lett. 1995, 240, 283.

[9] M. Sierka, A. Hogekamp, R. Ahlrichs, J. Chem. Phys. 2003, 118, 9136.

[10]A. Klamt, G. Schuurmann, J. Chem. Soc., Perkin Trans. 2 1993, 799-805.

[11]P. W. Ayers, R. G. Parr, R. G. Pearson, J. Chem. Phys. 2006, 124, 194107.

[12] J. L. Gazquez, F. Mendez, J. Phys. Chem. 1994, 98, 4591-4593.

[13]F. Mendez, J. L. Gazquez, J. Am. Chem. Soc. 1994, 116, 9298-9301.

[14]C. Gatti, P. Macchi, Modern Charge Density Analysis, 2012, 715-764.

[15] S. Kozuch, J. M. L. Martin, ACS Catal. 2011, 1, 246.

[16]A. Uhe, S. Kozuch, S. Shaik, J. Comput. Chem. 2010, 32, 978.

[17] S. Kozuch, WIREs Comput. Mol. Sci. 2012, 2, 795.

[18]A. Uhe, S. Kozuch, S. Shaik, J. Comput. Chem. 2011, 32, 978.

[19] S. Kozuch, S. Shaik, Acc. Chem. Res. 2010, 44, 101.

[20]W. Li, X. Liu, X. Hao, X. Hu, Y. Chu, W. Cao, S. Qin, C. Hu, L. Lin, X. Feng, J. Am. Chem. Soc. 2011, 133, 15268-15271. 
13. Copies of ${ }^{1} \mathrm{H}$ and ${ }^{13} \mathrm{C}$ NMR spectra

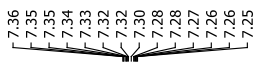

诲

$\stackrel{\circ}{i}$<smiles>C#CCOC(=O)Cc1ccccc1</smiles>

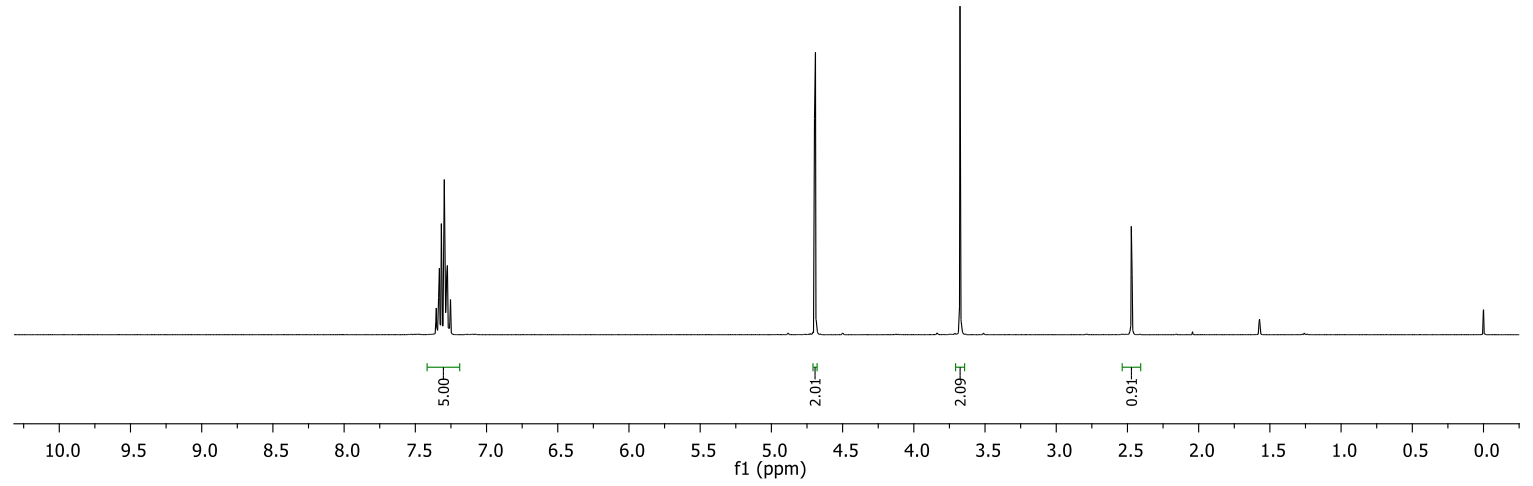



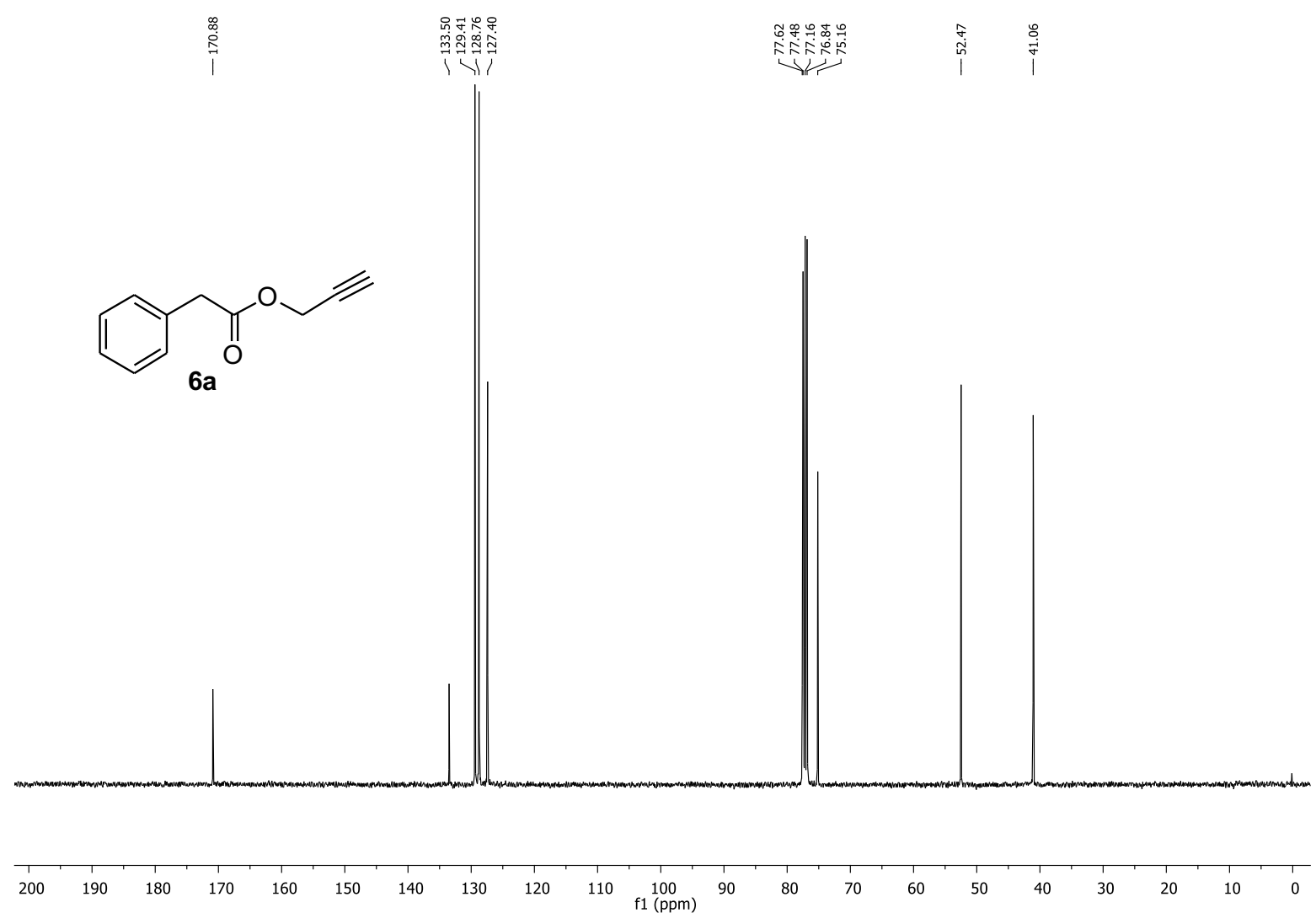

रॉ<smiles>C#CCOC(=O)Cc1ccc(Br)cc1</smiles>

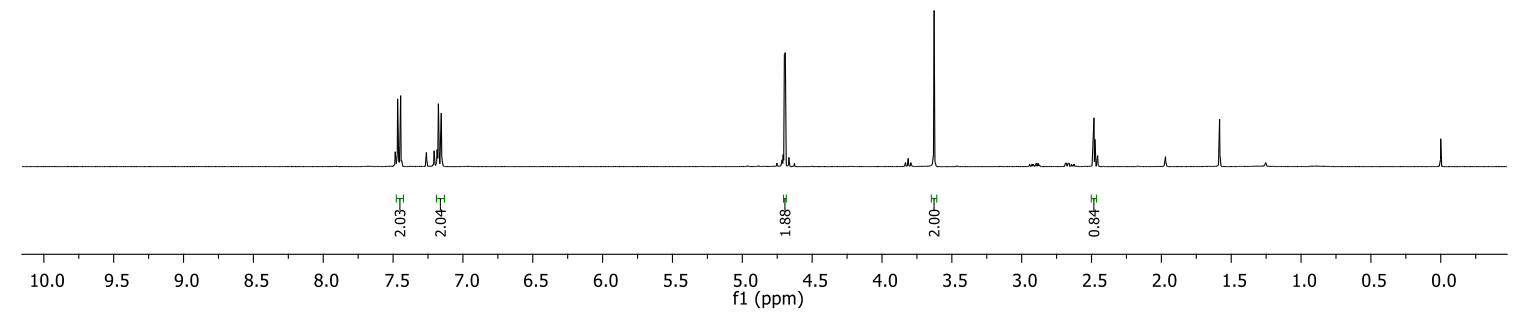




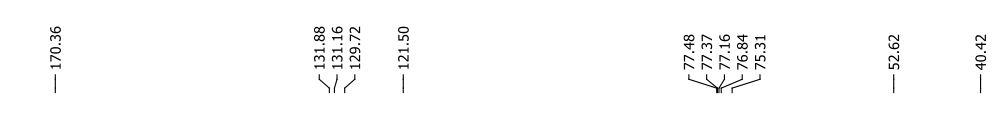<smiles>C#CCOC(=O)Cc1ccc(Br)cc1</smiles>
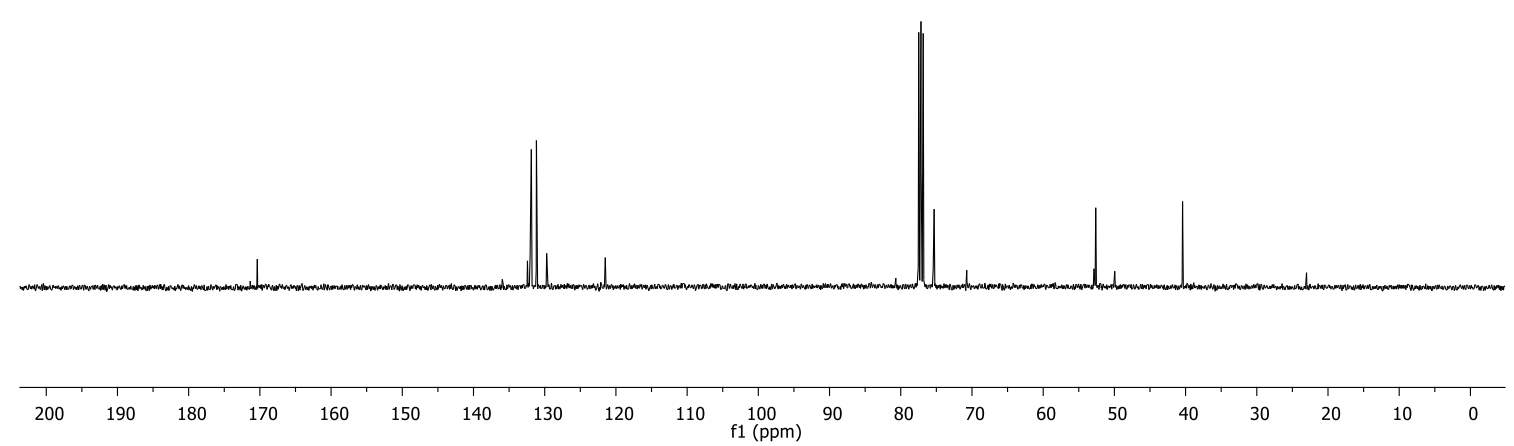

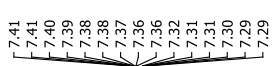

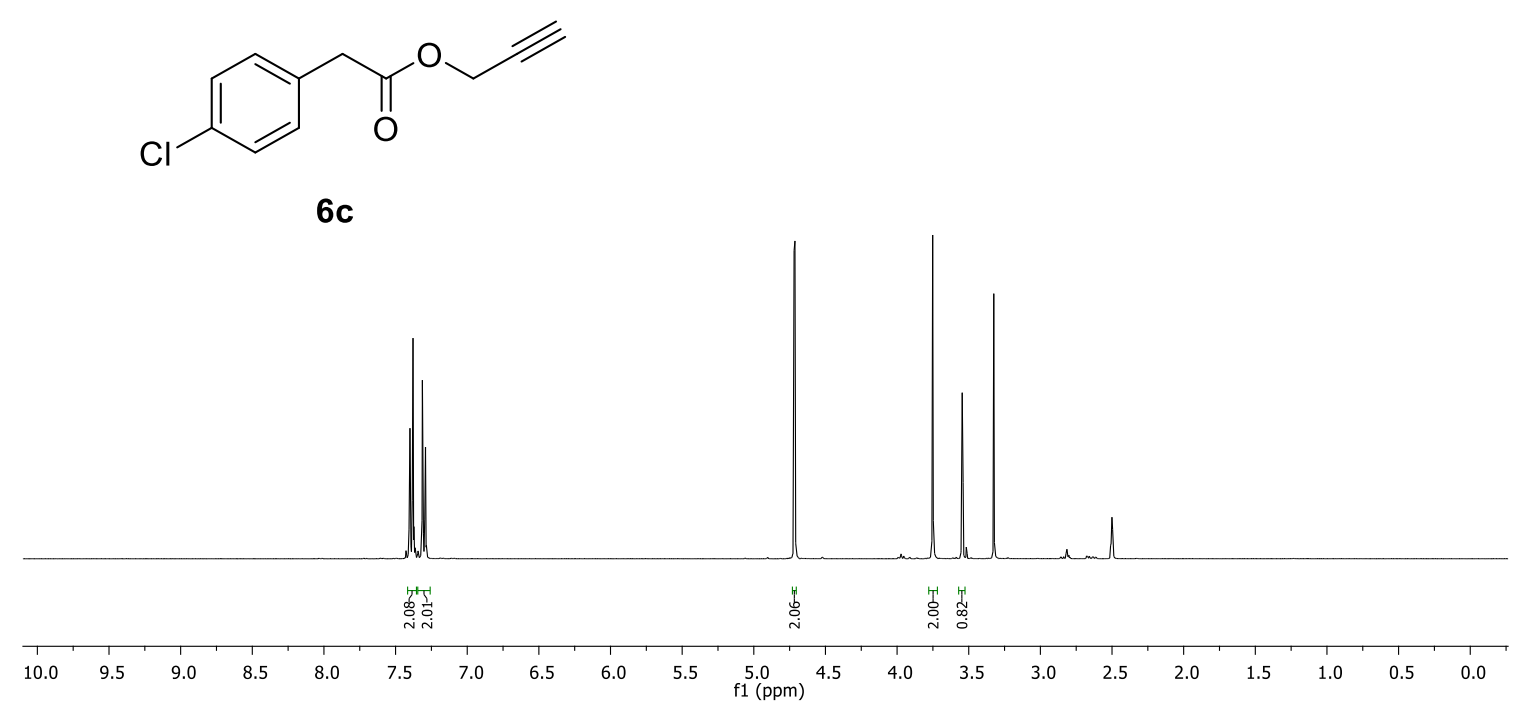




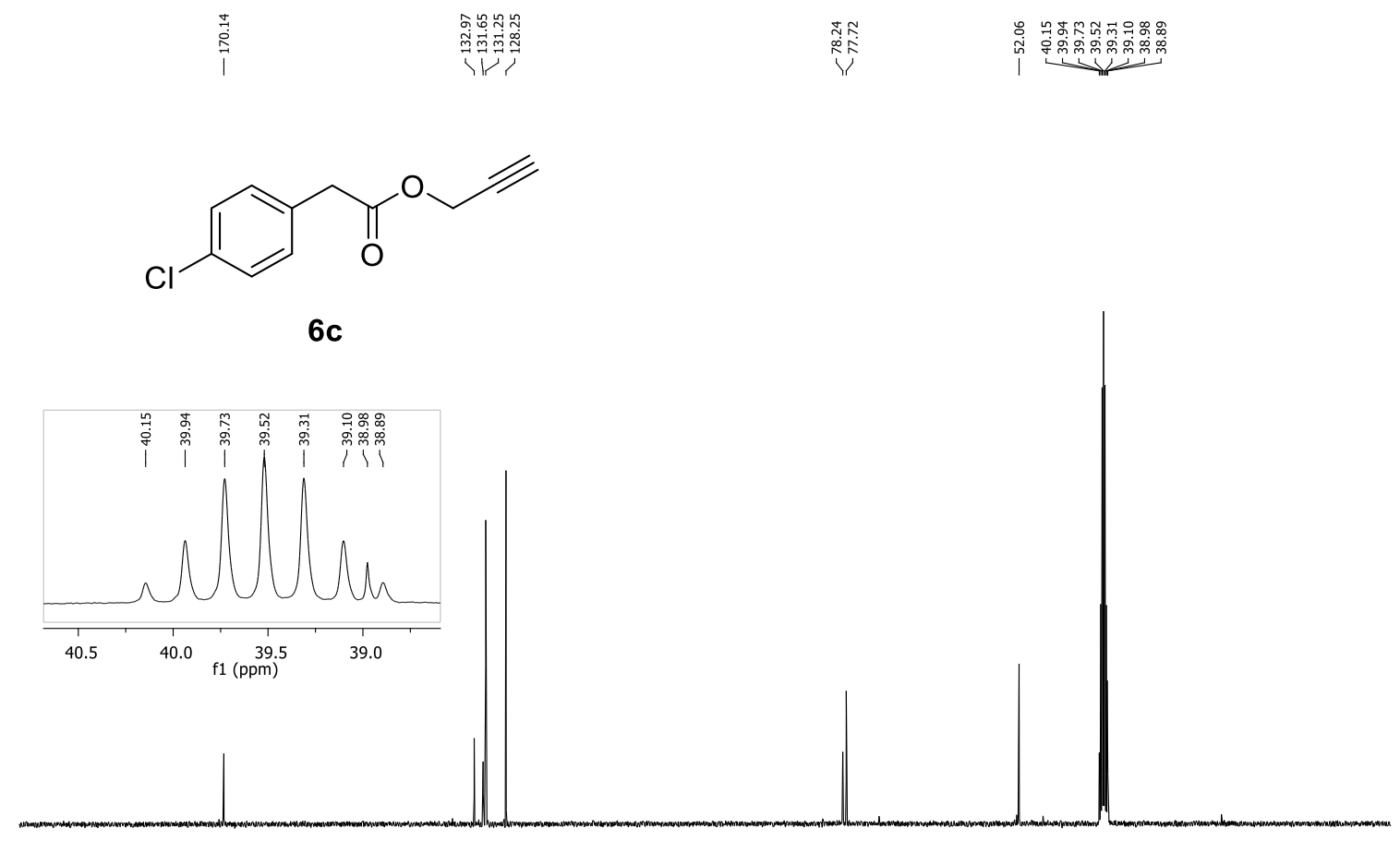

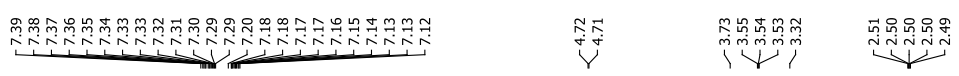

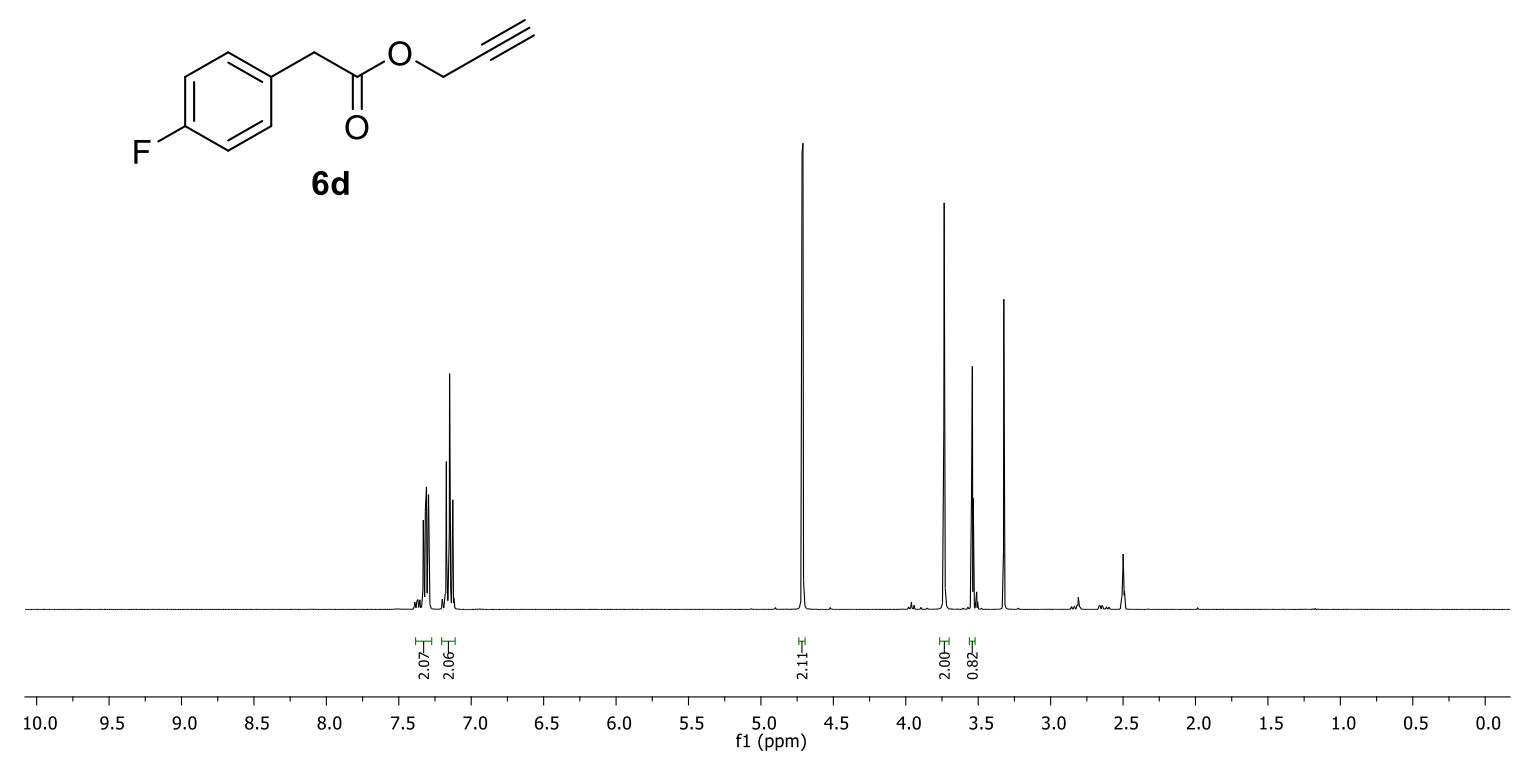




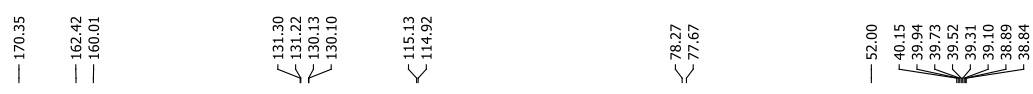<smiles>C#CCOC(=O)Cc1ccc(F)cc1</smiles>
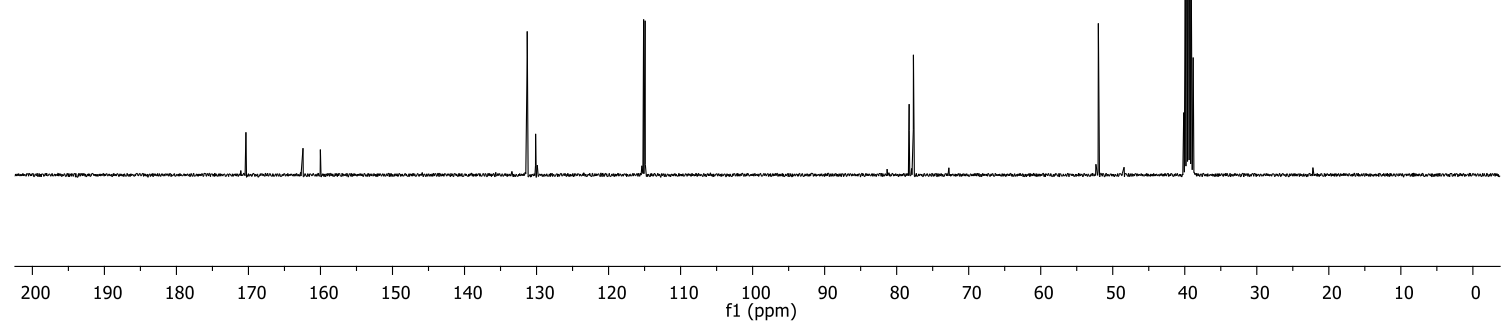

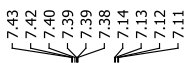

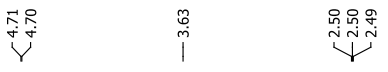<smiles>C#CCOC(=O)Cc1ccc(Cl)c(Cl)c1</smiles>

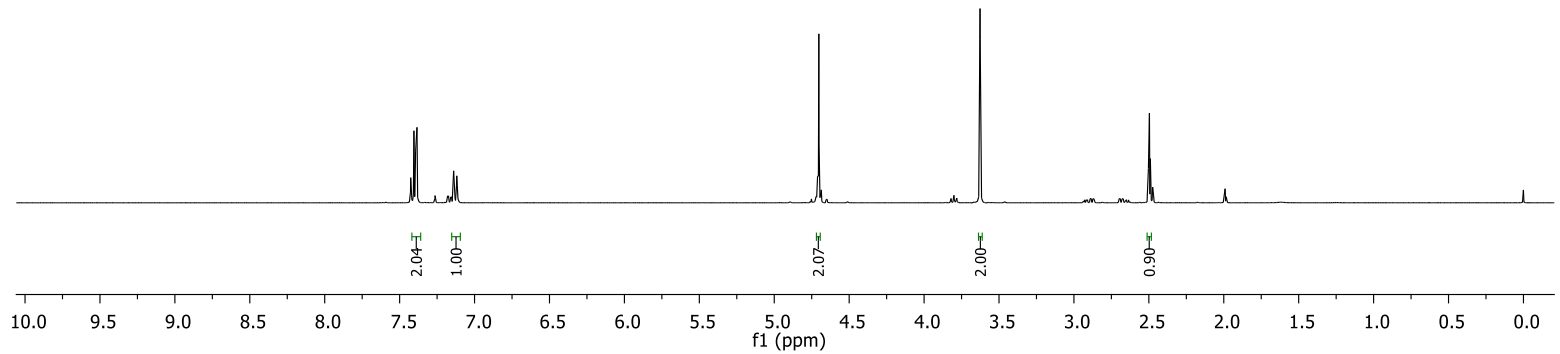




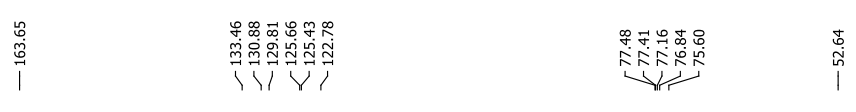<smiles>C#CCOC(=O)Cc1ccc(Cl)c(Cl)c1</smiles>

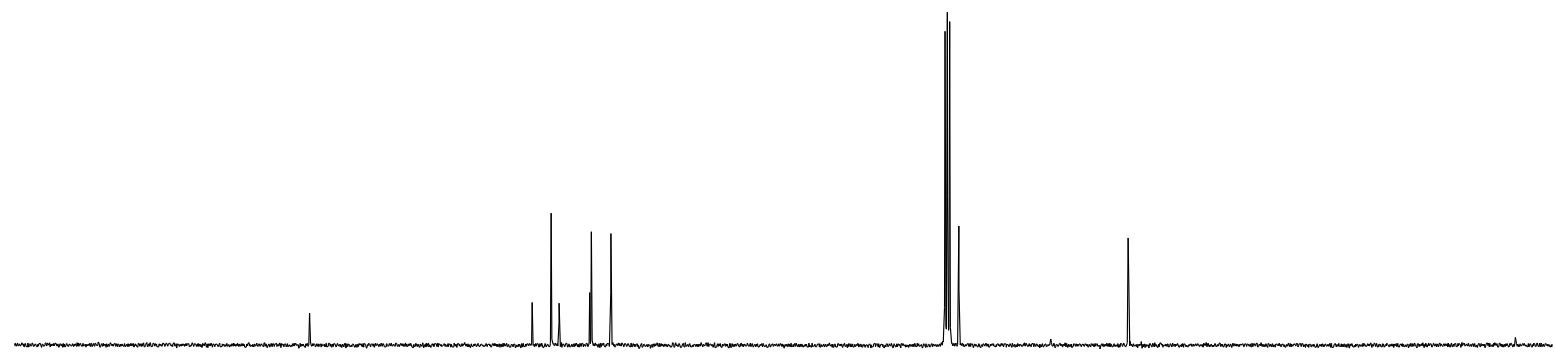

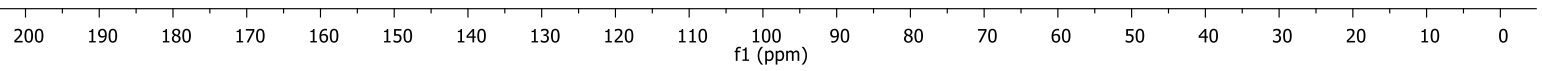

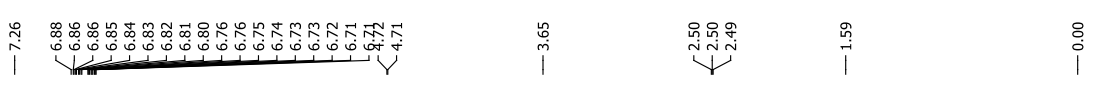

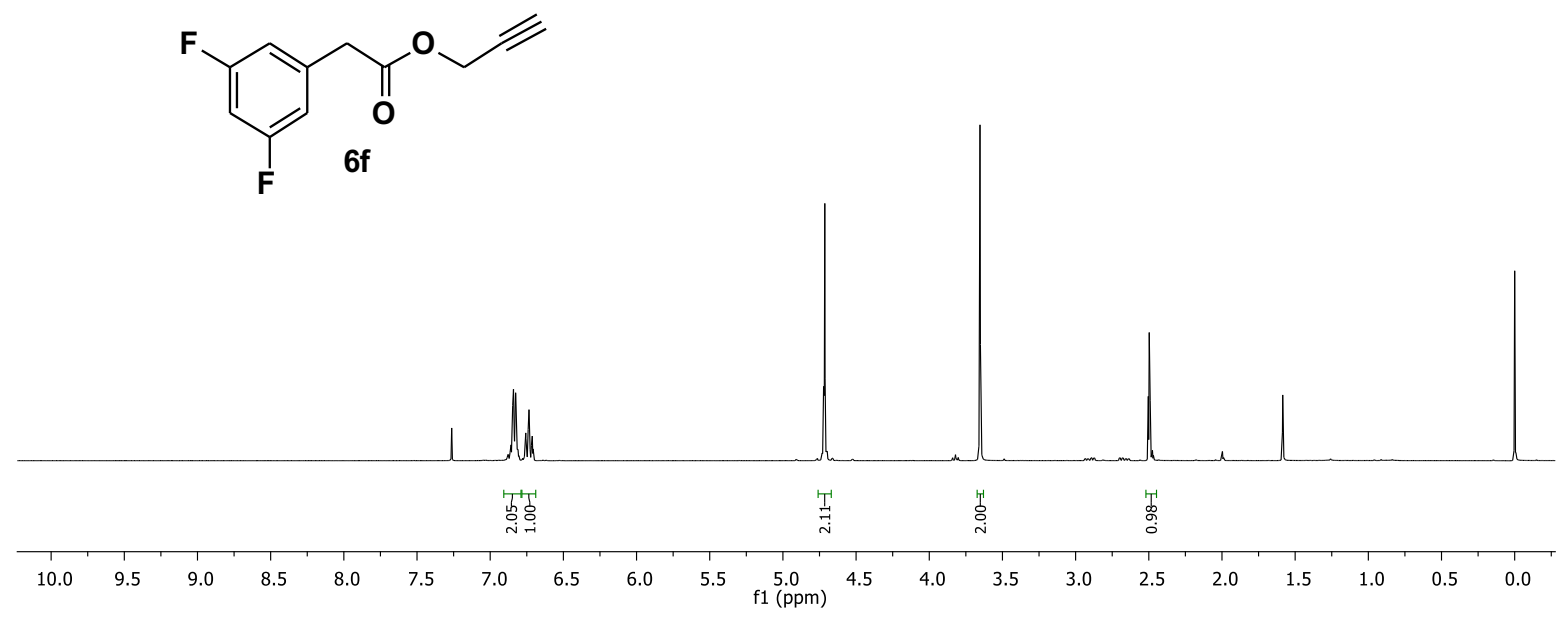




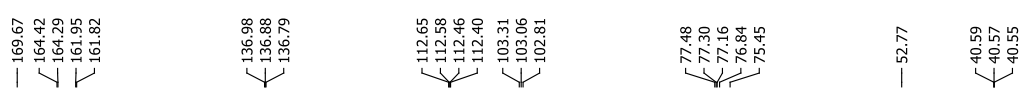<smiles>C#CCOC(=O)Cc1cc(F)cc(F)c1</smiles>

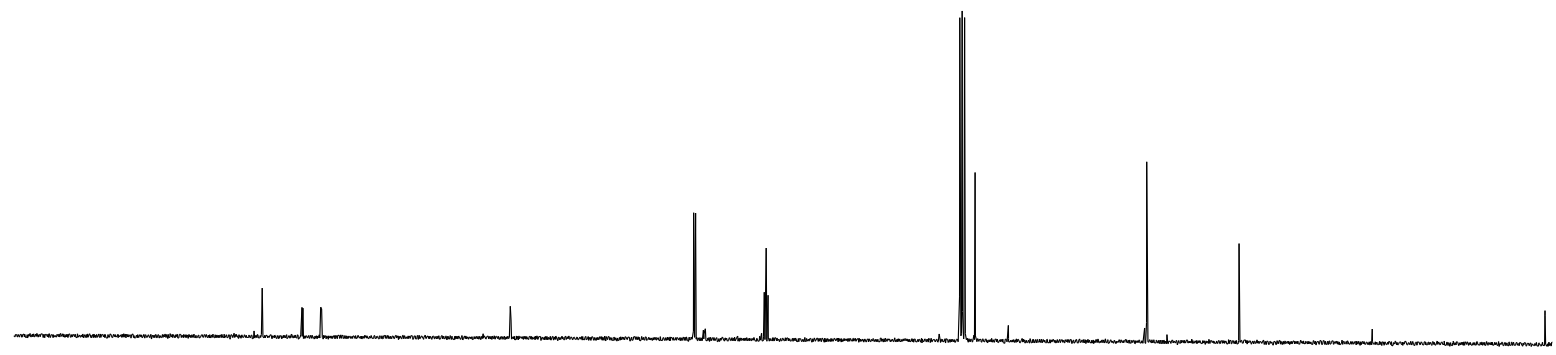

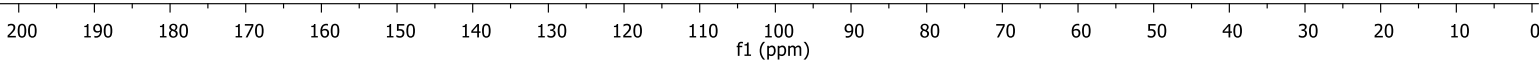

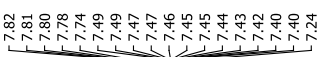

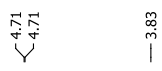

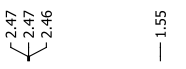<smiles>C#CCOC(=O)Cc1ccc2ccccc2c1</smiles>

$6 \mathrm{~g}$

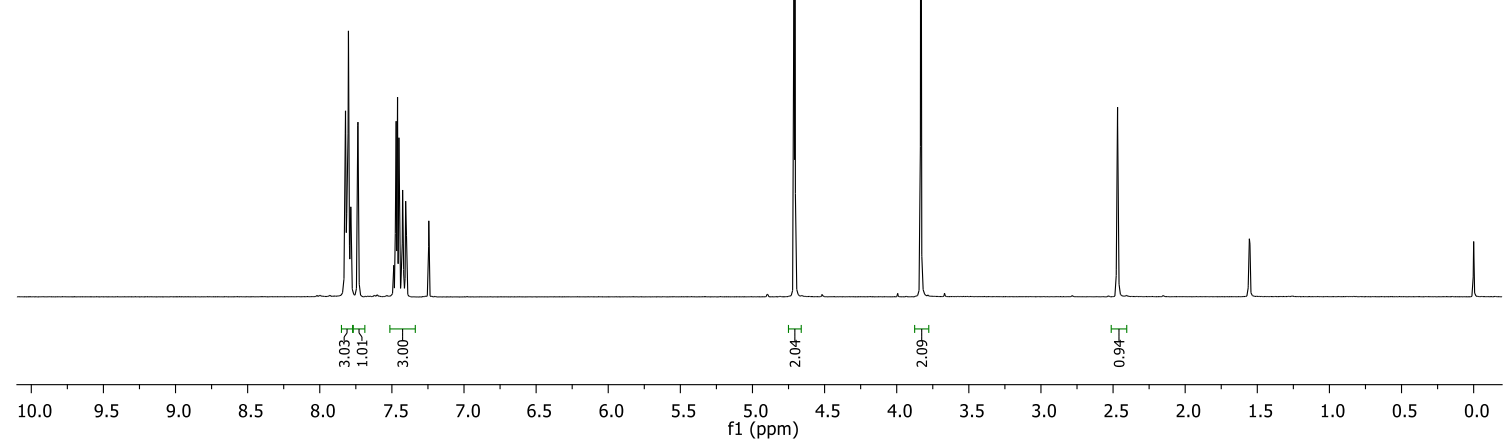



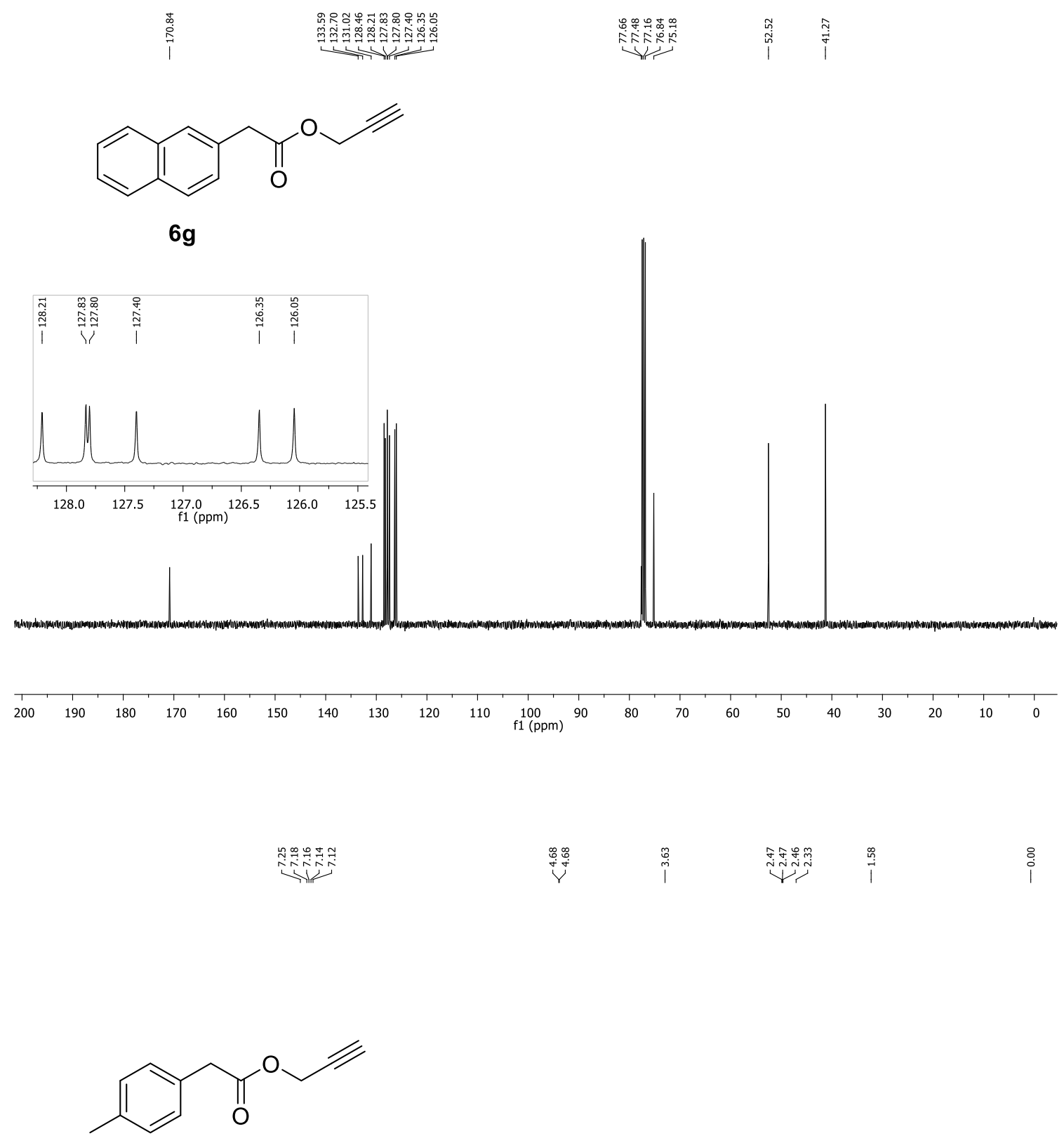

$6 h$

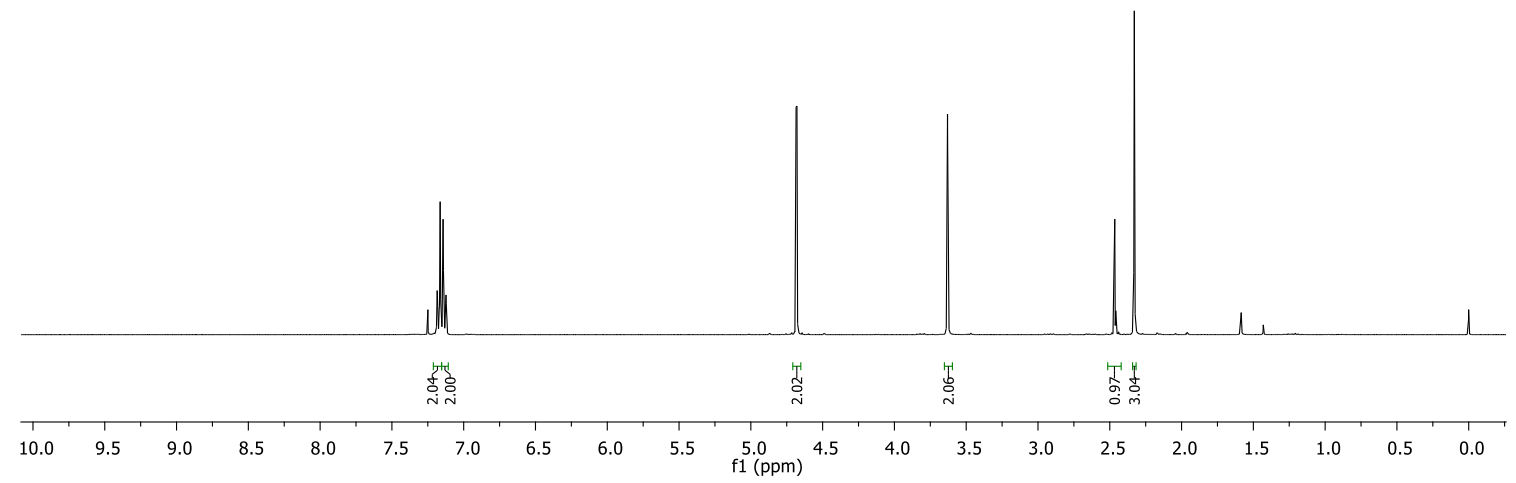



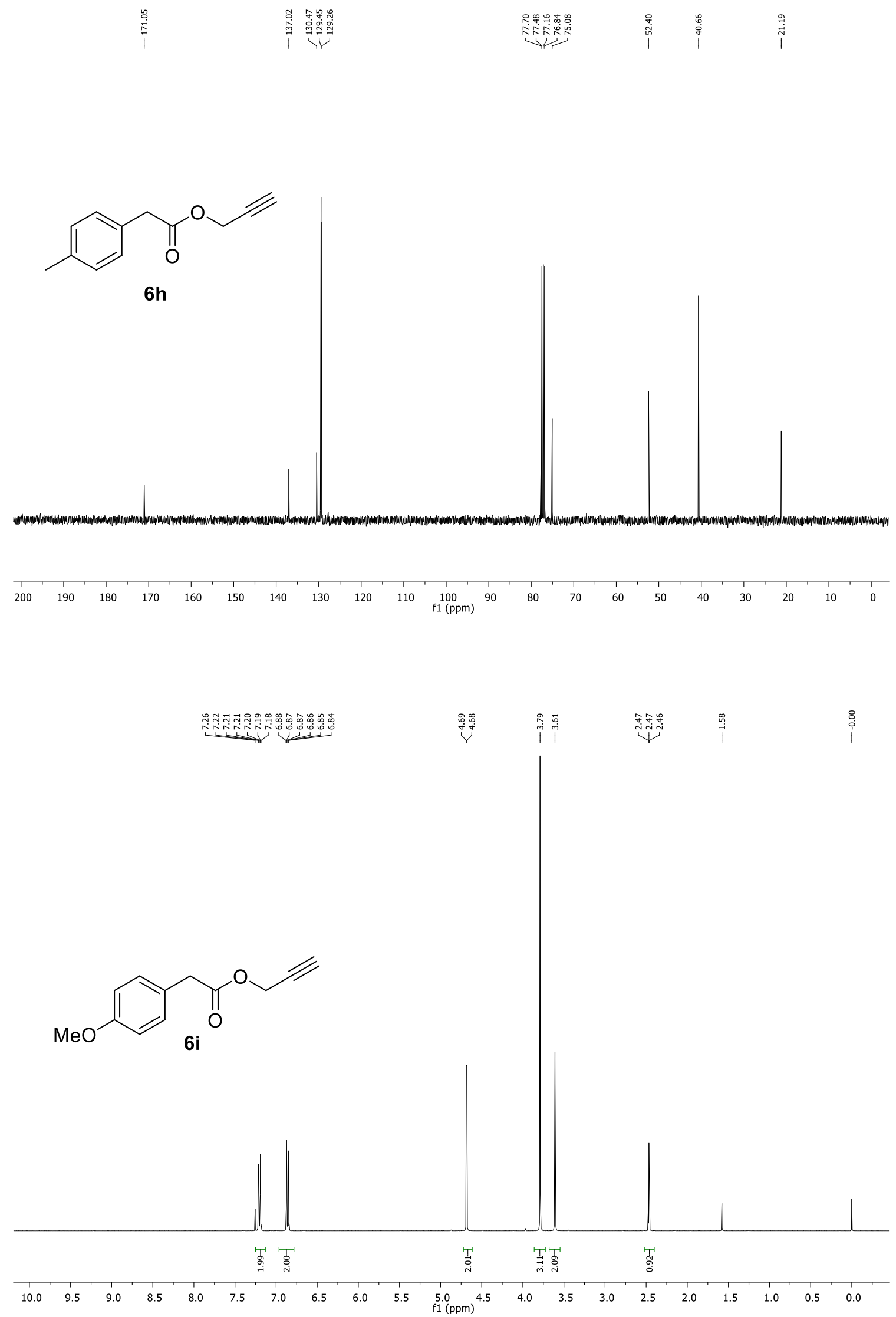


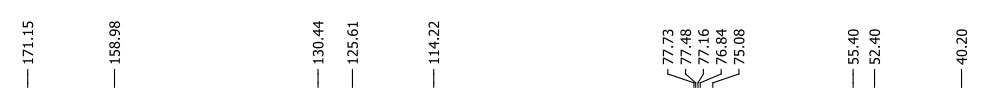

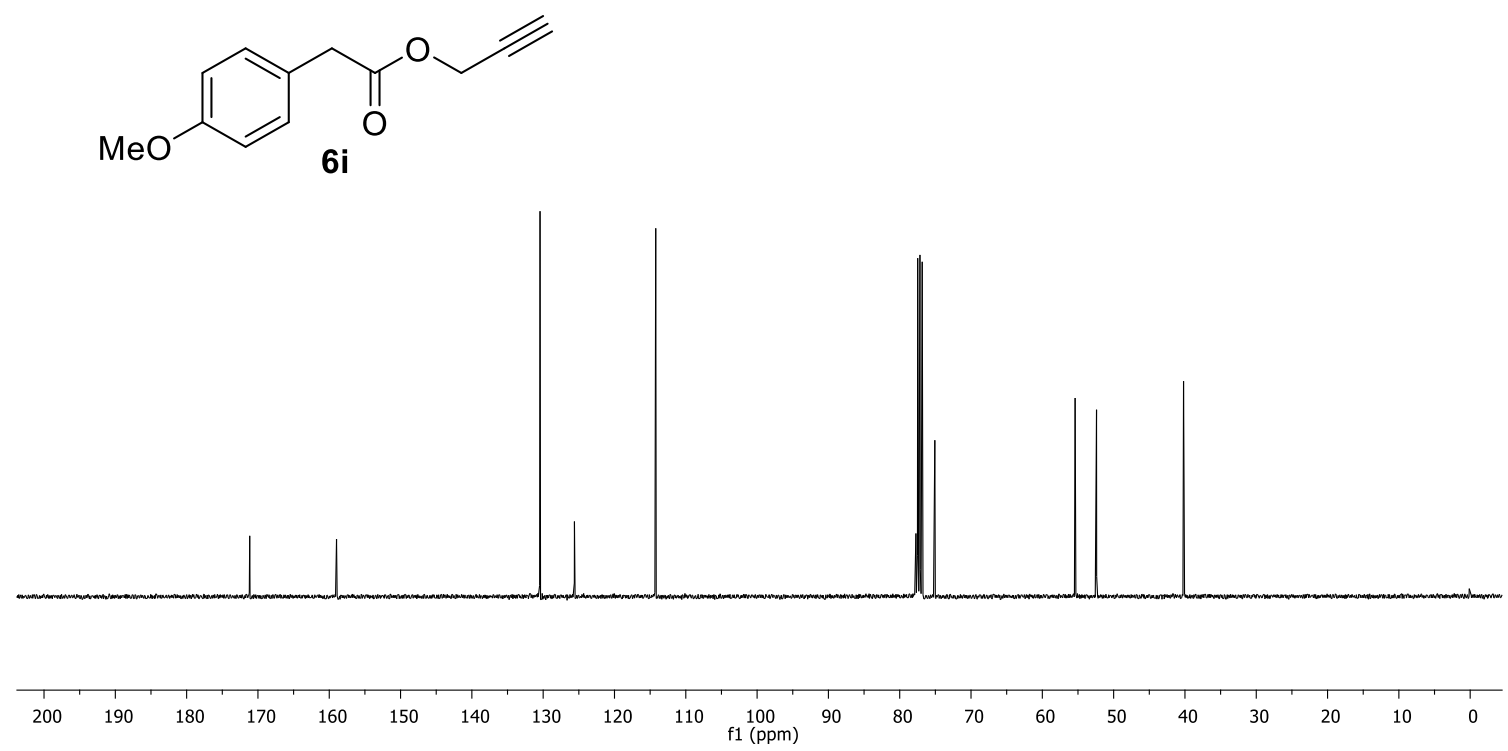



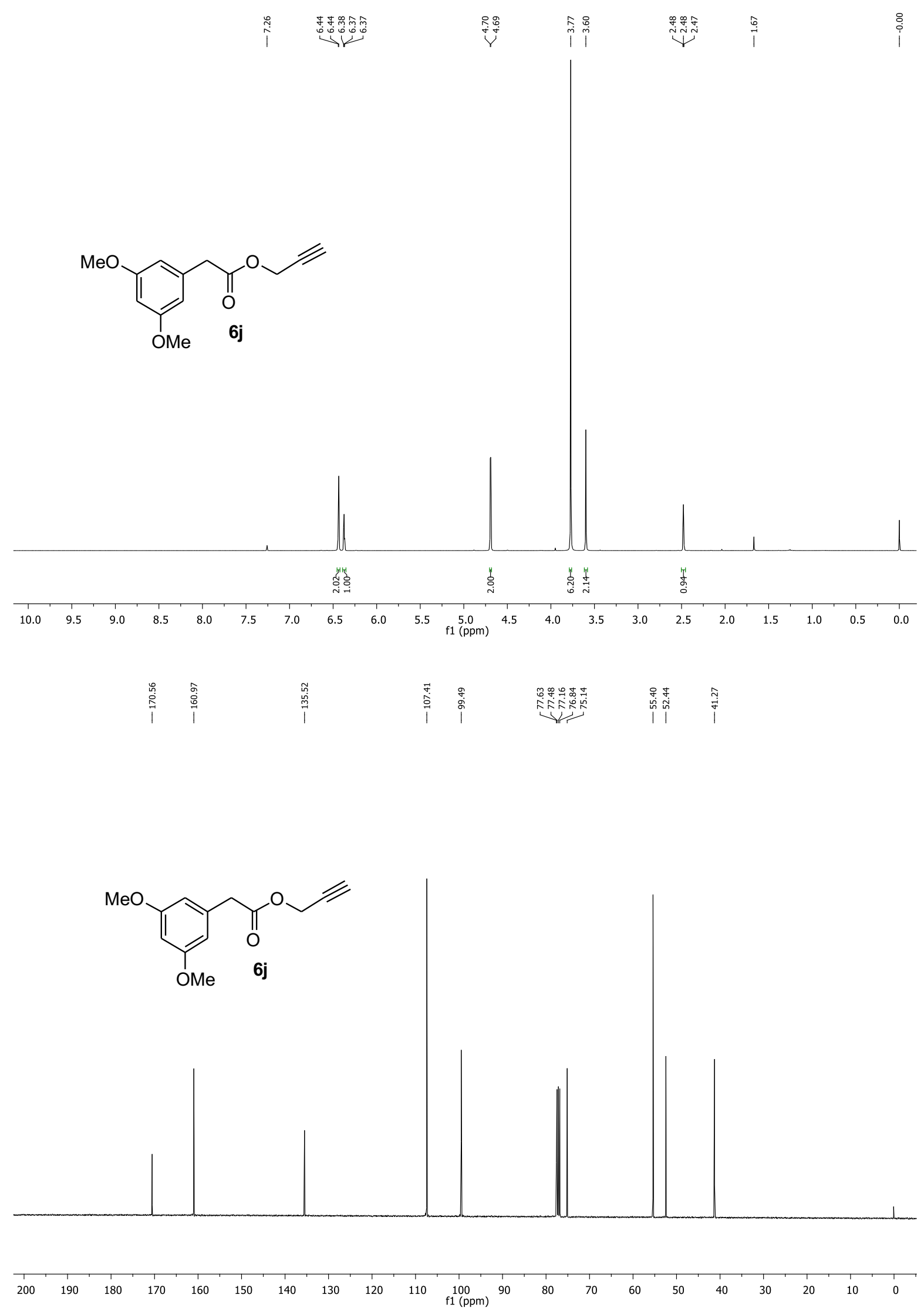

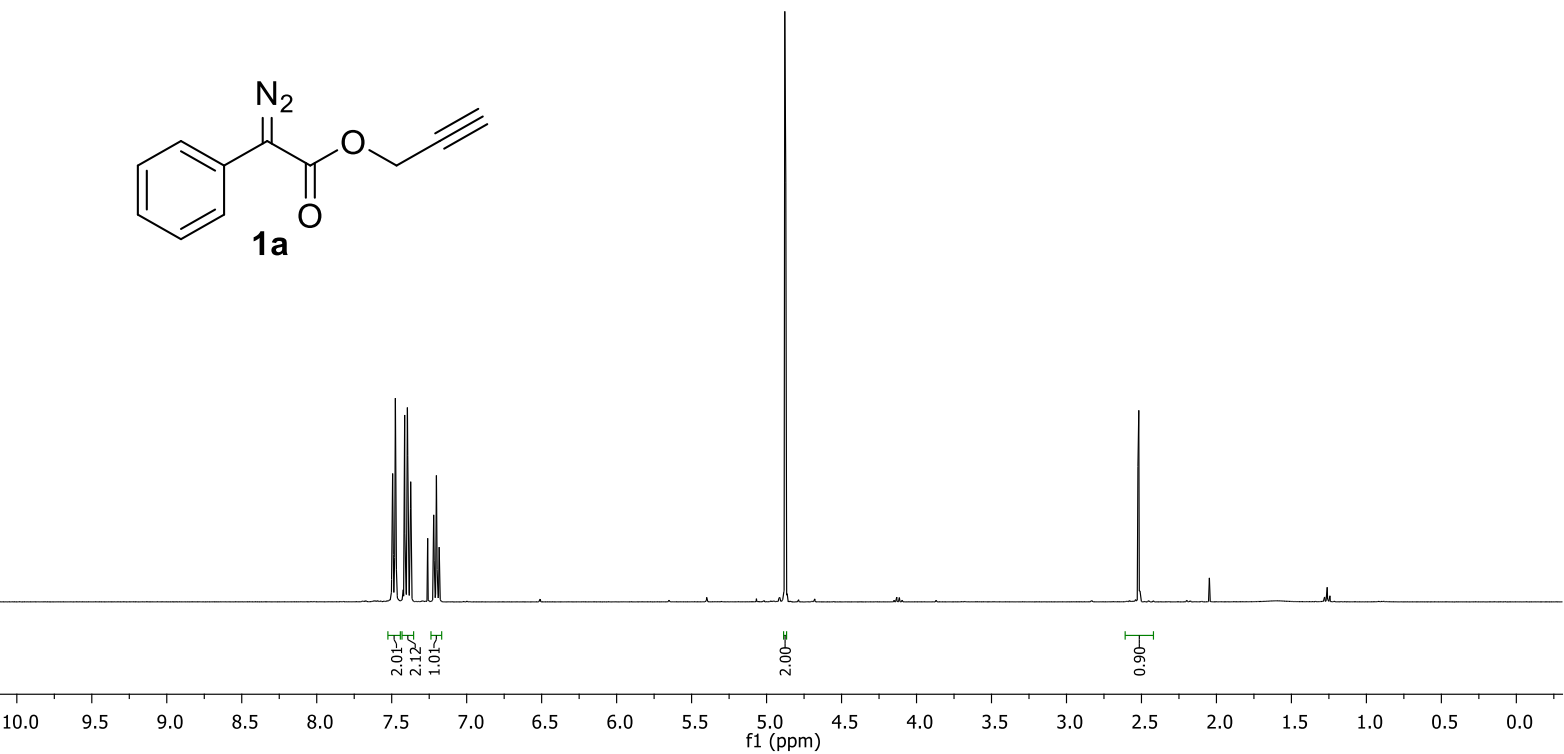

\begin{tabular}{|c|c|}
\hline & 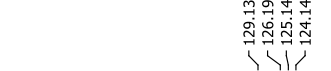 \\
\hline
\end{tabular}

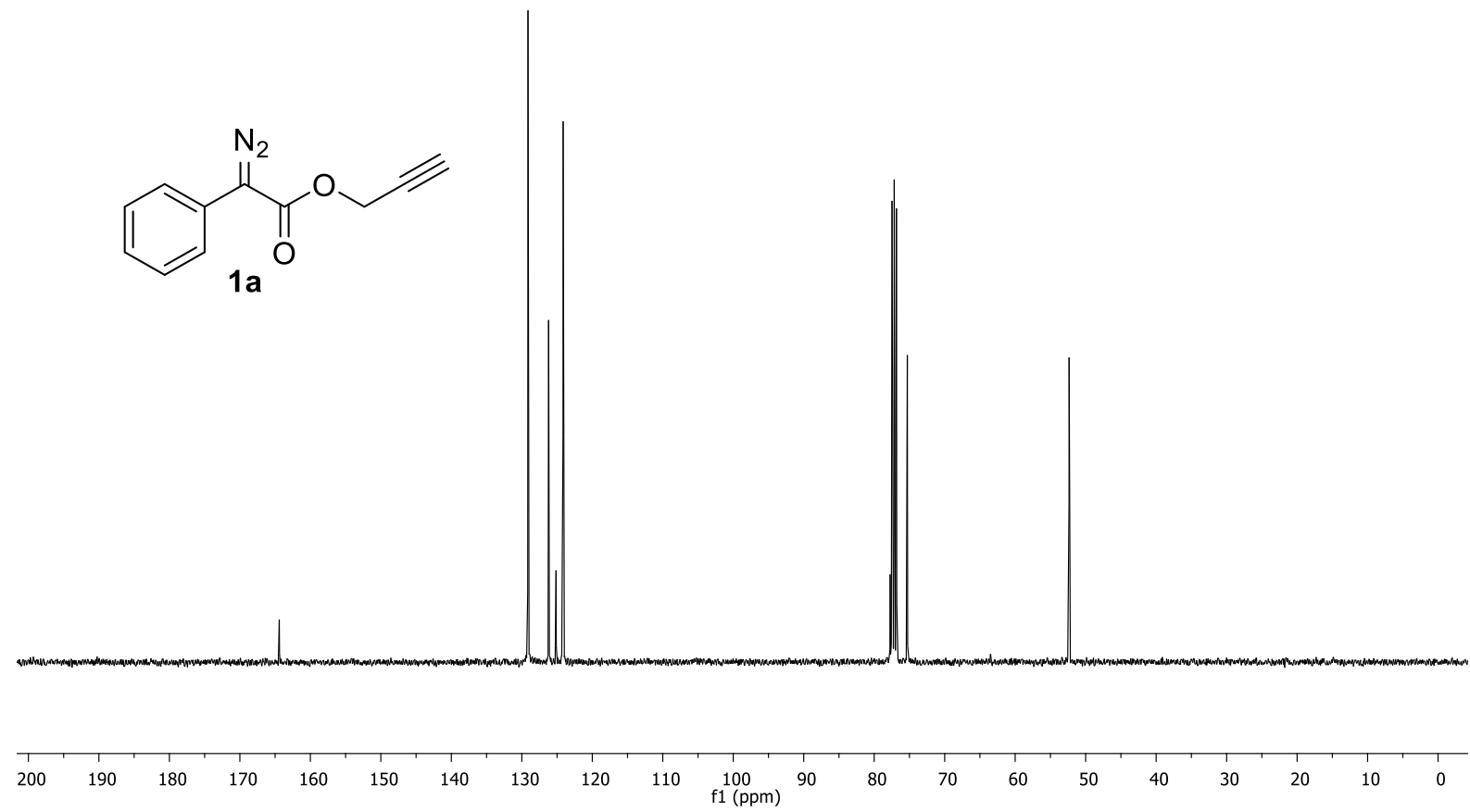


<smiles>CCOC(=O)C(=N)C(=[18O])c1ccccc1</smiles>
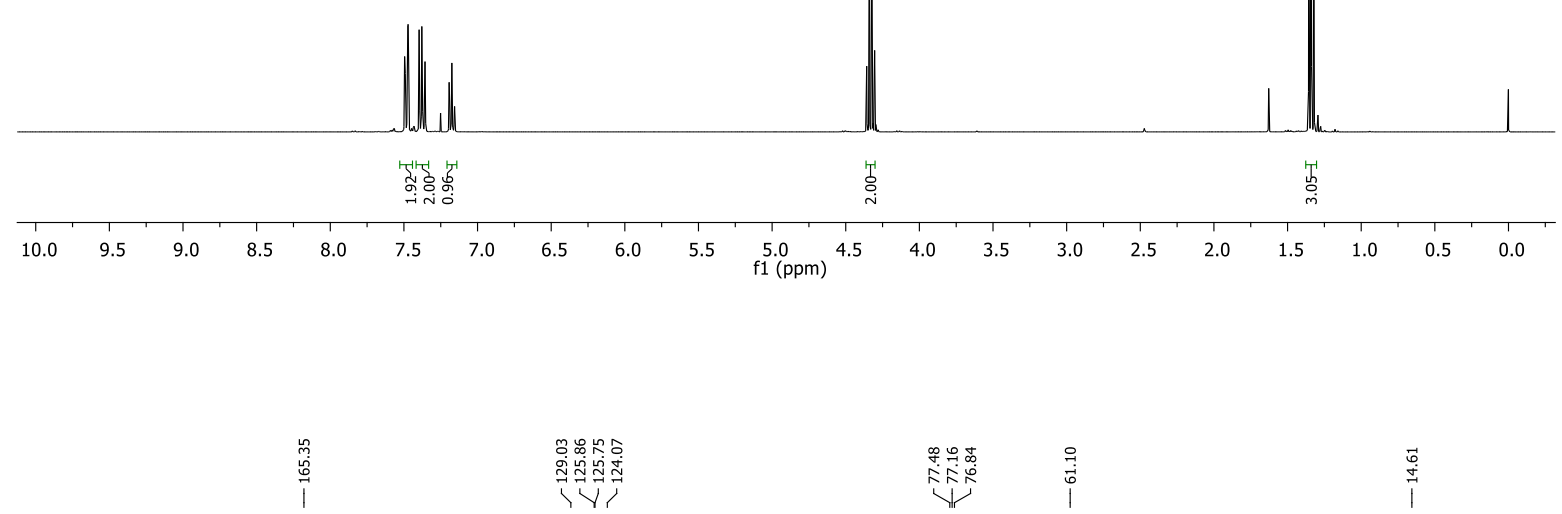

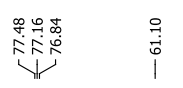

$\stackrel{\vec{b}}{\dot{\sigma}}$<smiles>CCOC(=O)C(=N)c1ccccc1</smiles>

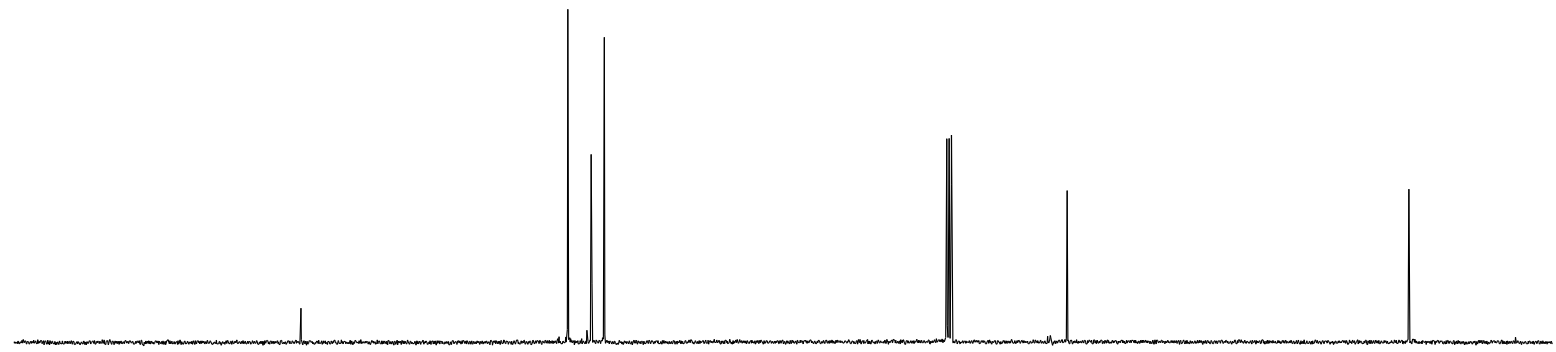

\begin{tabular}{lllllllllllllllllllllll}
\hline 200 & 190 & 180 & 170 & 160 & 150 & 140 & 130 & 120 & 110 & 100 & 90 & 80 & 70 & 60 & 50 & 40 & 30 & 20 & 10 & 0
\end{tabular} 
<smiles>C=CCOC(=O)C(=N)c1ccccc1</smiles>

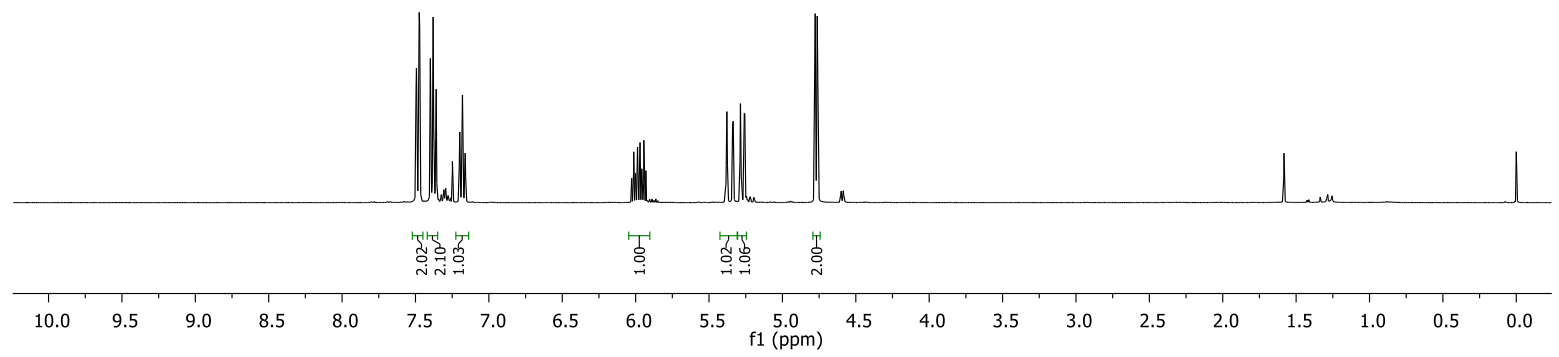

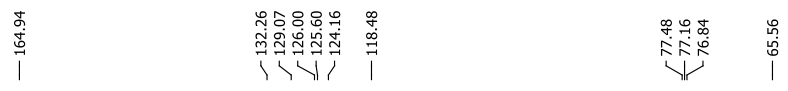<smiles>C=COC(=O)C(=N)c1ccccc1</smiles>

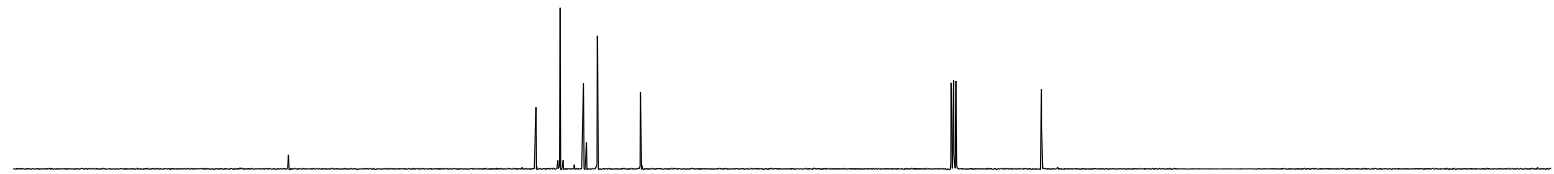

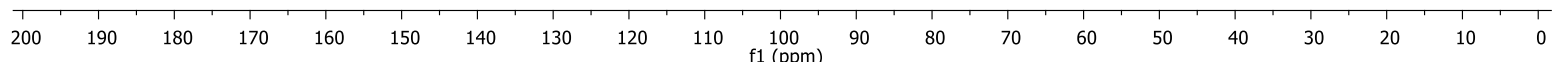


<smiles>N#CC(=O)OCC(F)(F)F</smiles>
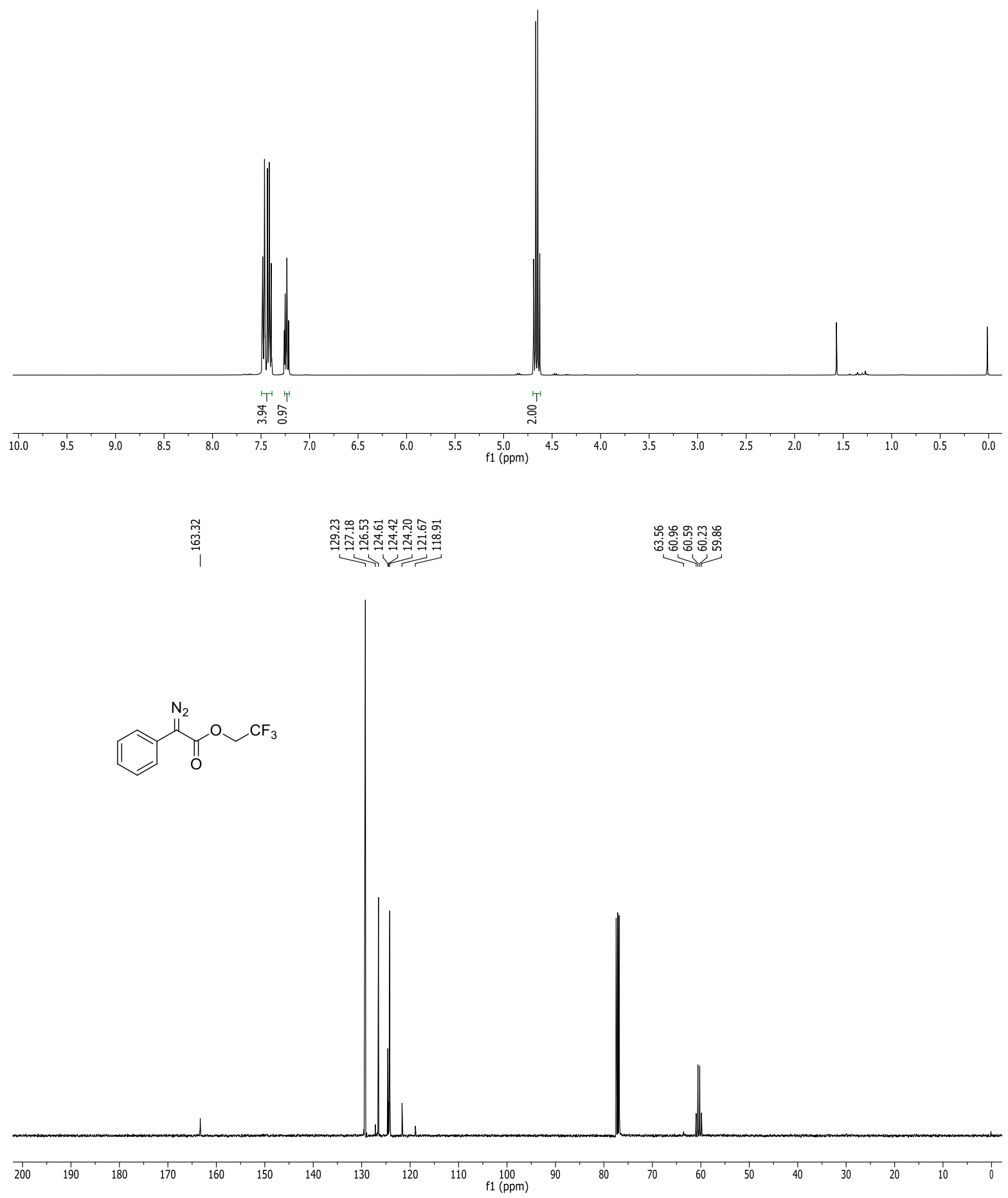


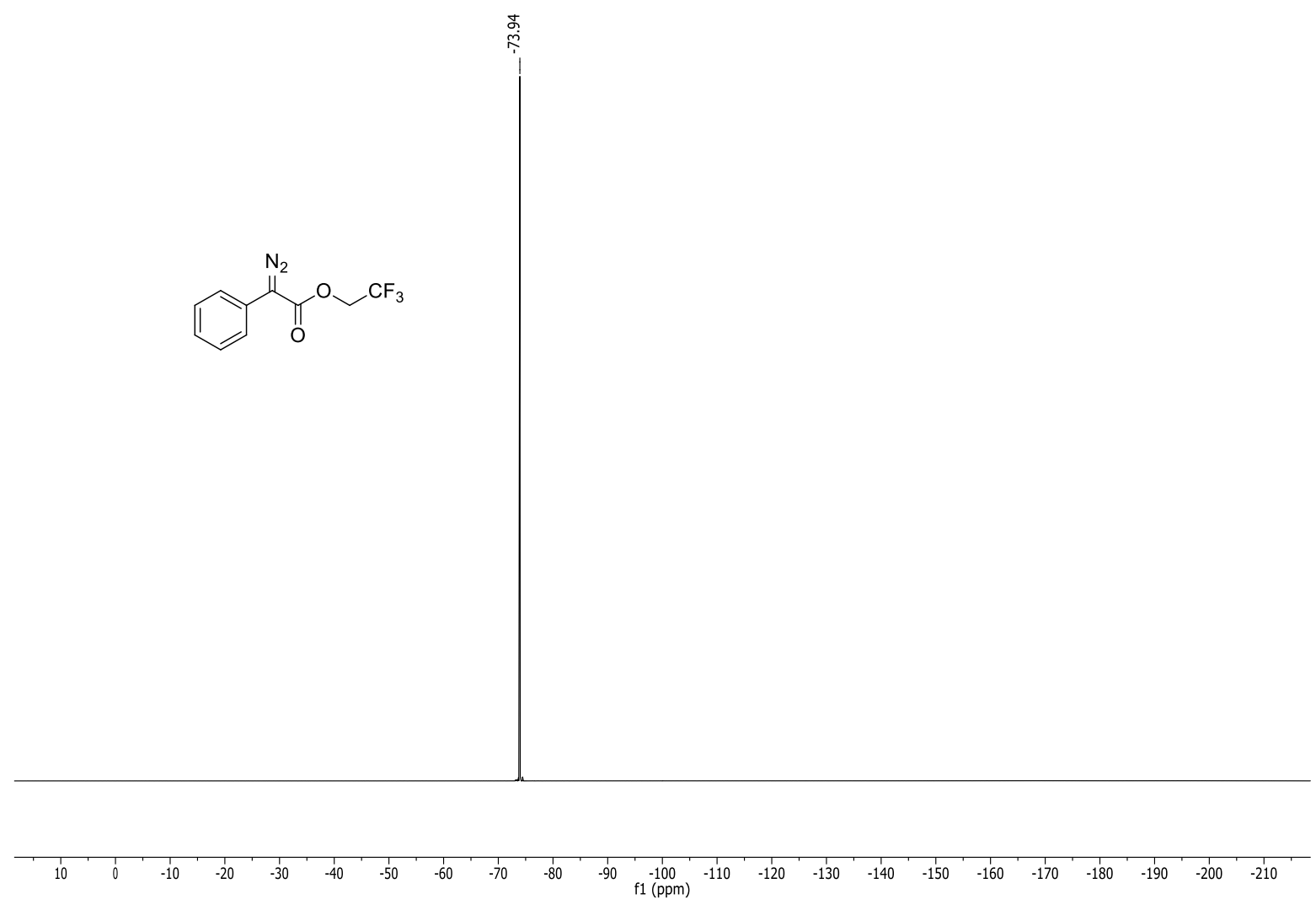



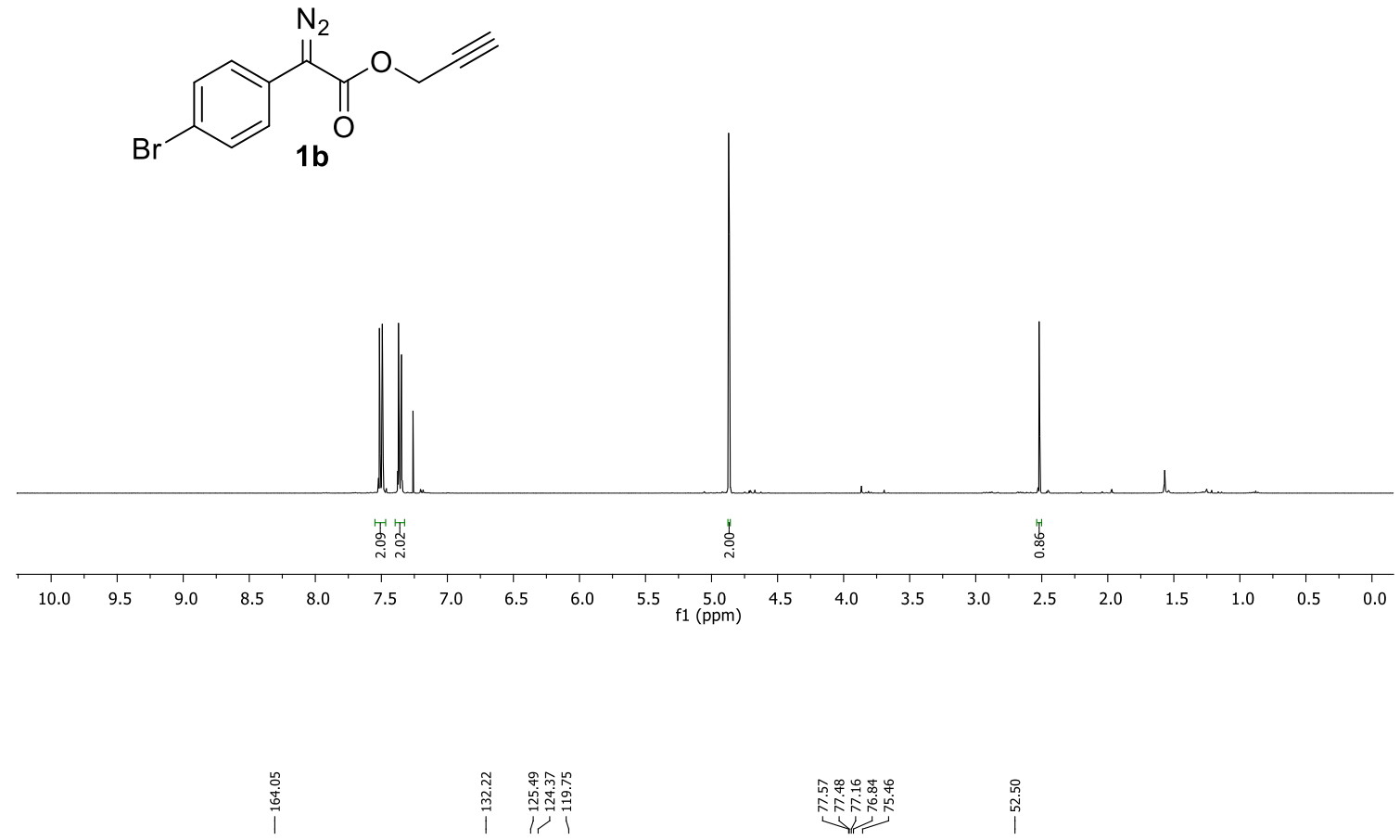

|

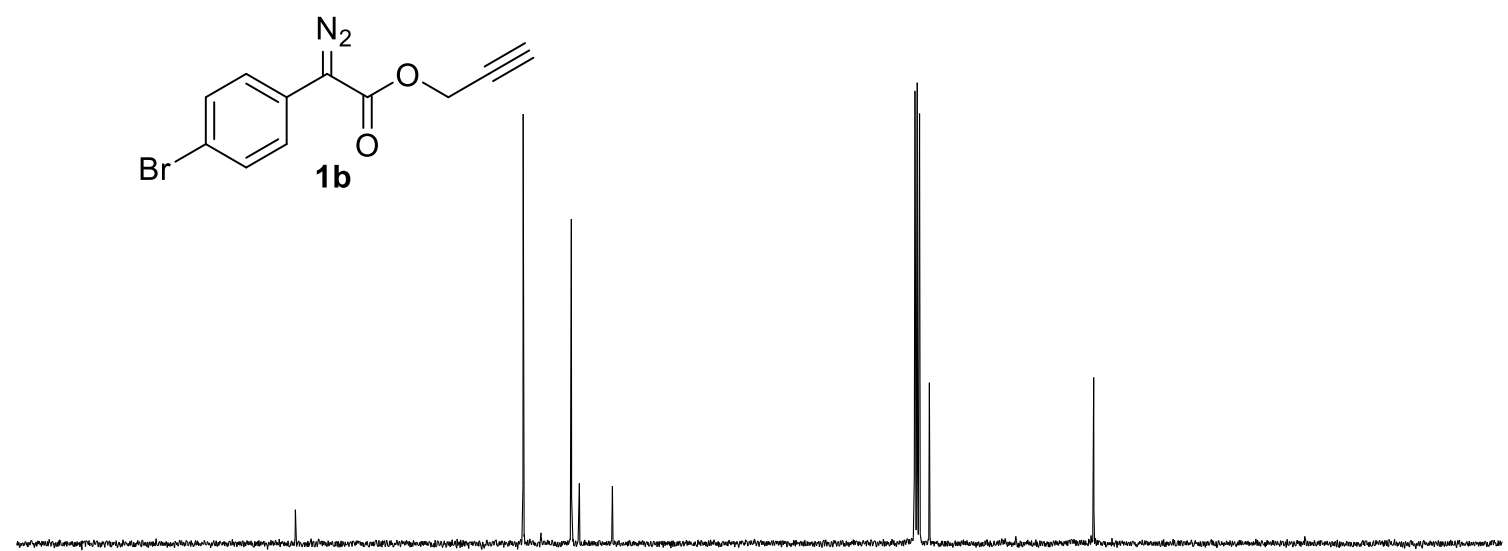

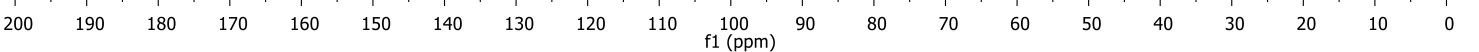



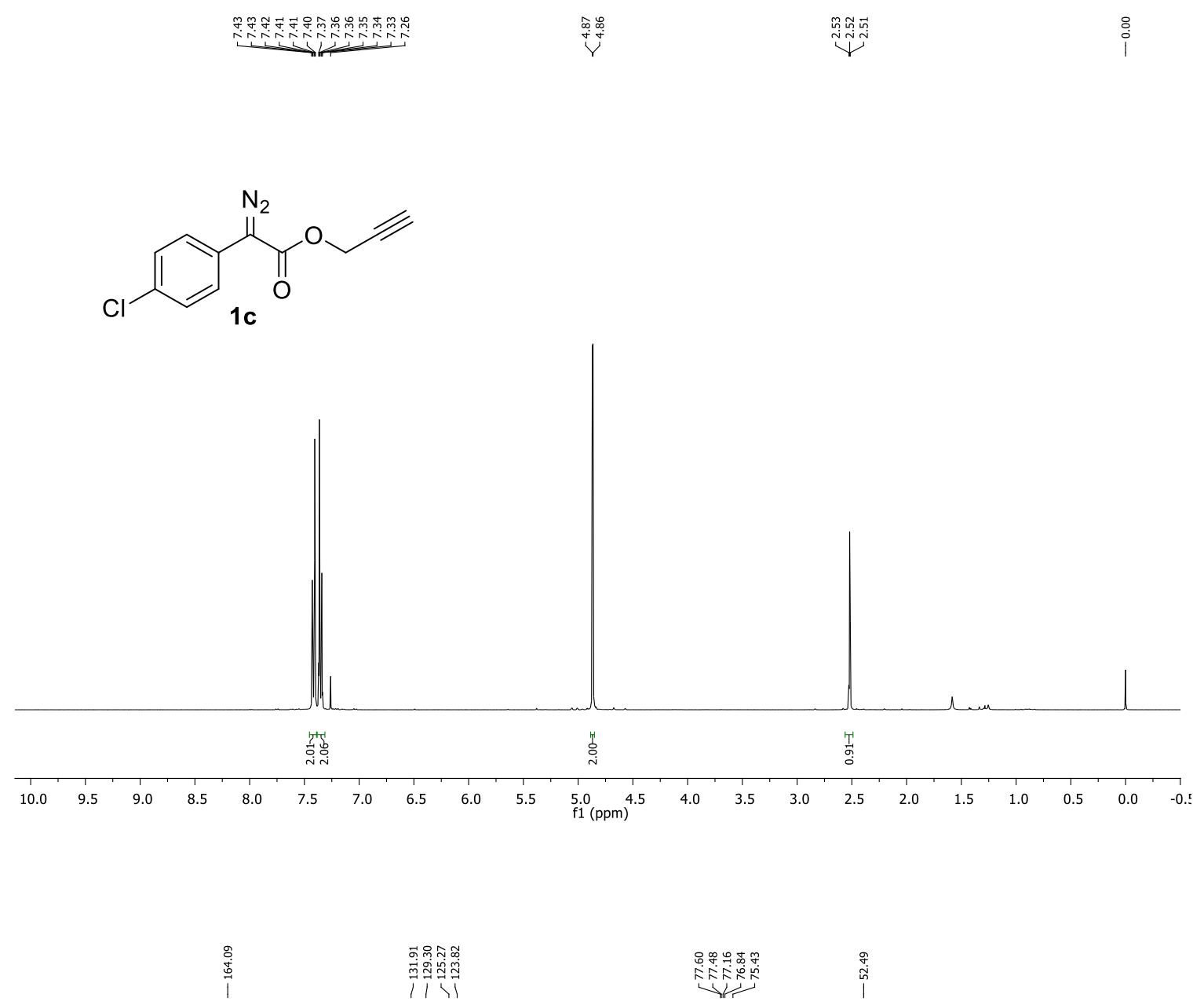

诲

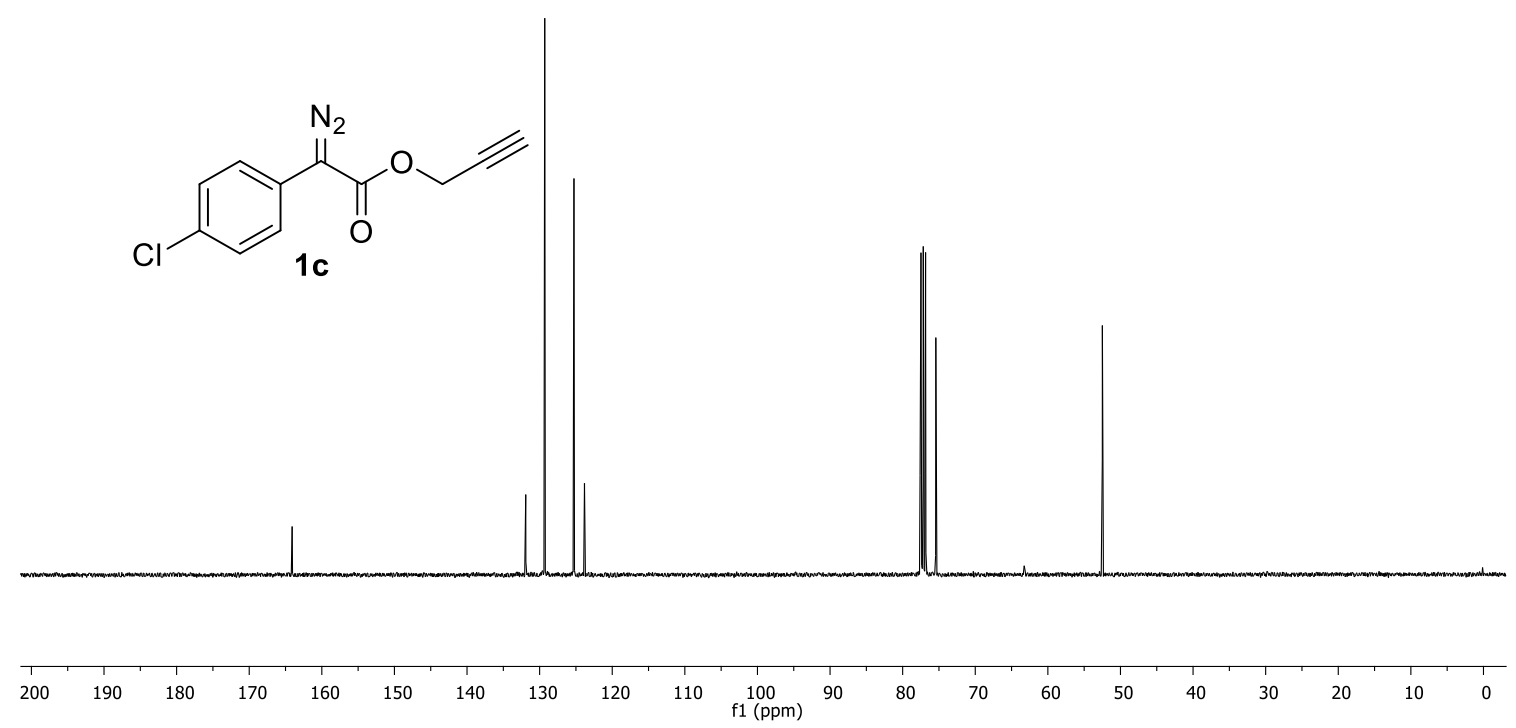


<smiles>C#CCOC(=O)C(=N)c1ccc(F)cc1</smiles>

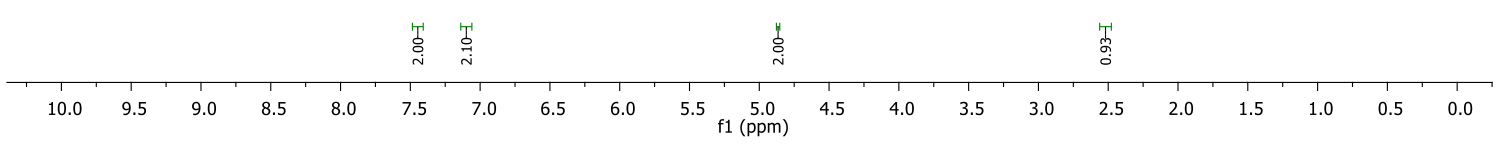

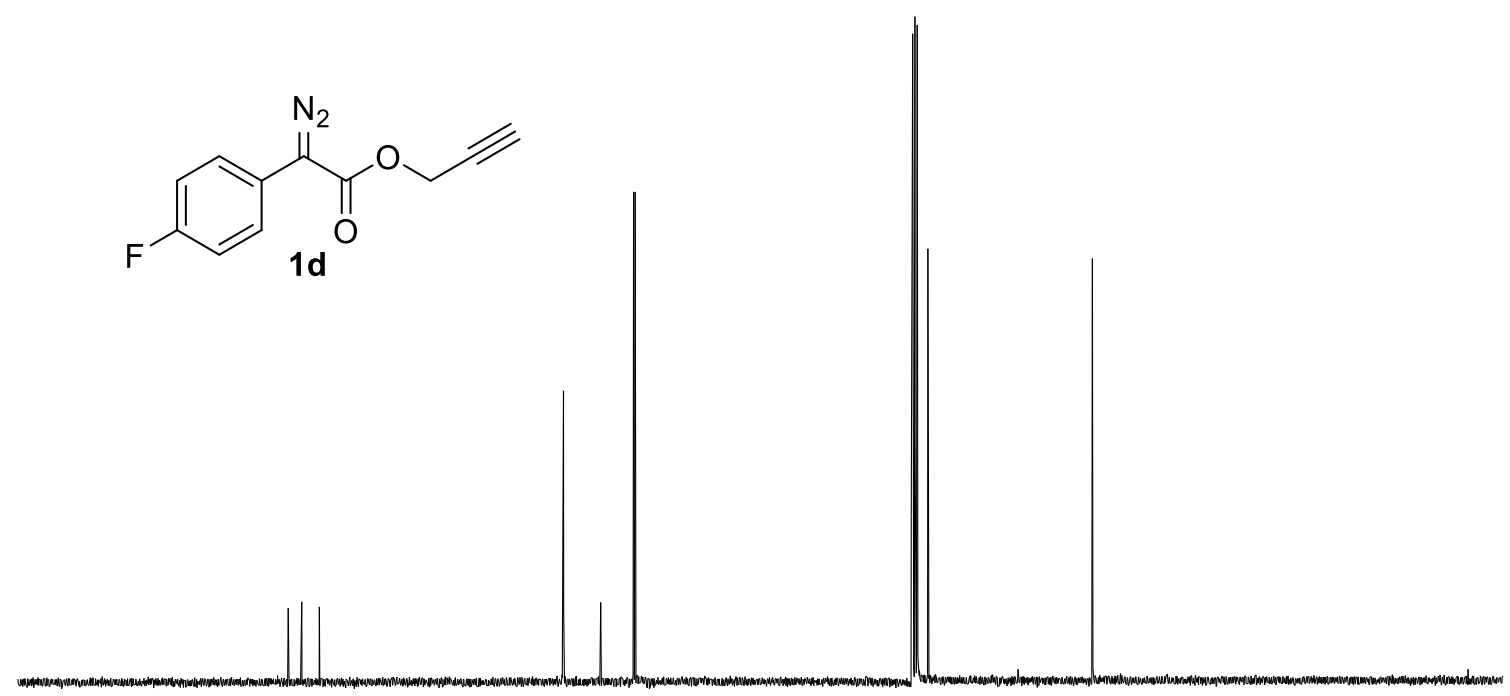

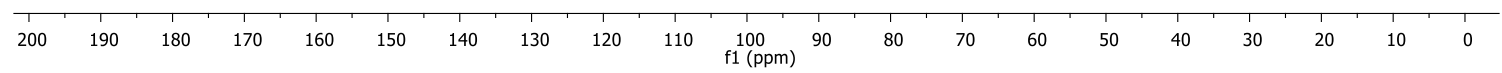


<smiles>C#CCOC(=O)C(=N)c1ccc(Cl)c(Cl)c1</smiles>
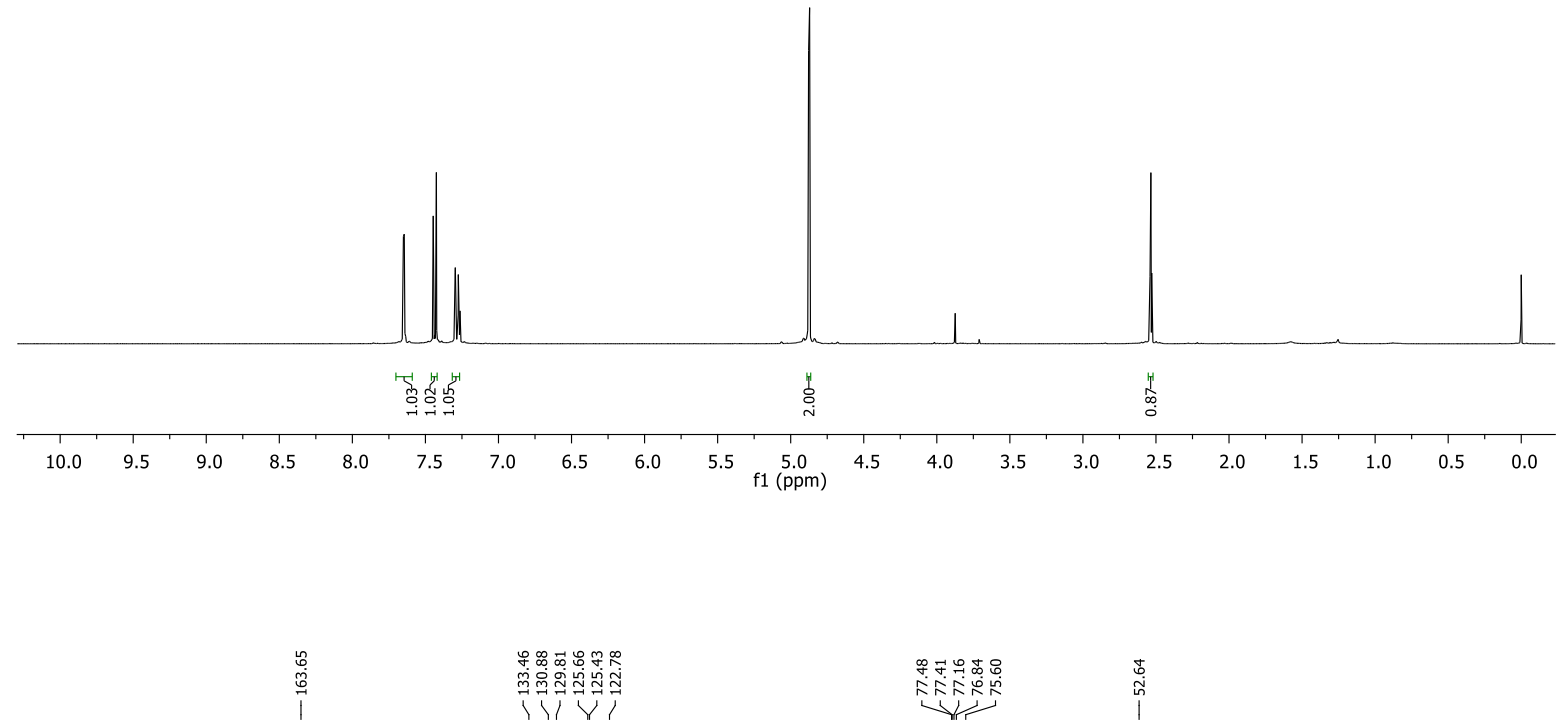<smiles>C#CCOC(=O)C(=N)c1ccc(Cl)c(Cl)c1</smiles>
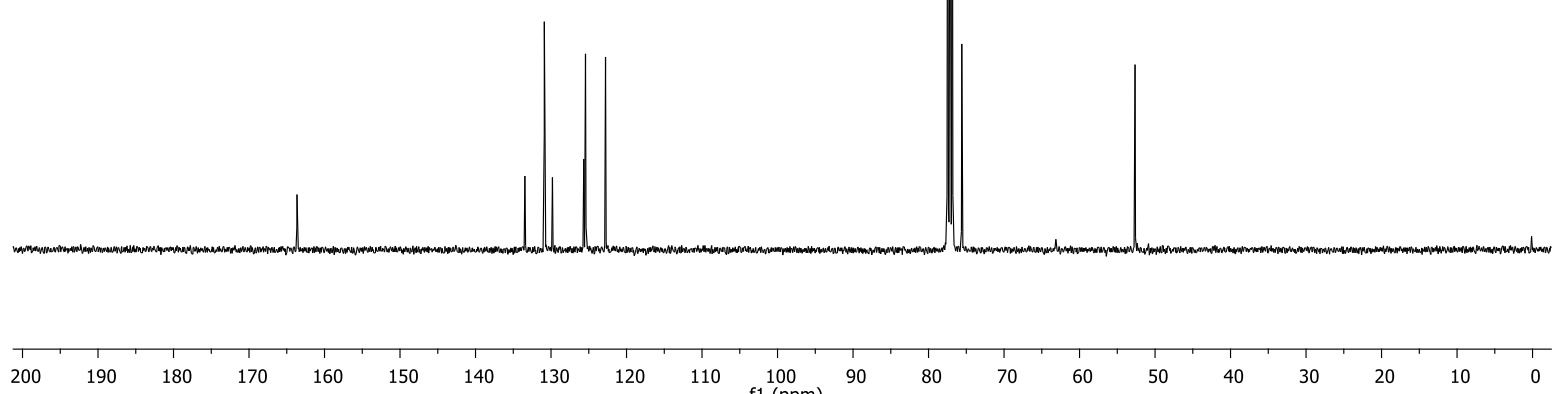
<smiles>C#CCOC(=O)C(=N)c1cc(F)cc(F)c1</smiles>

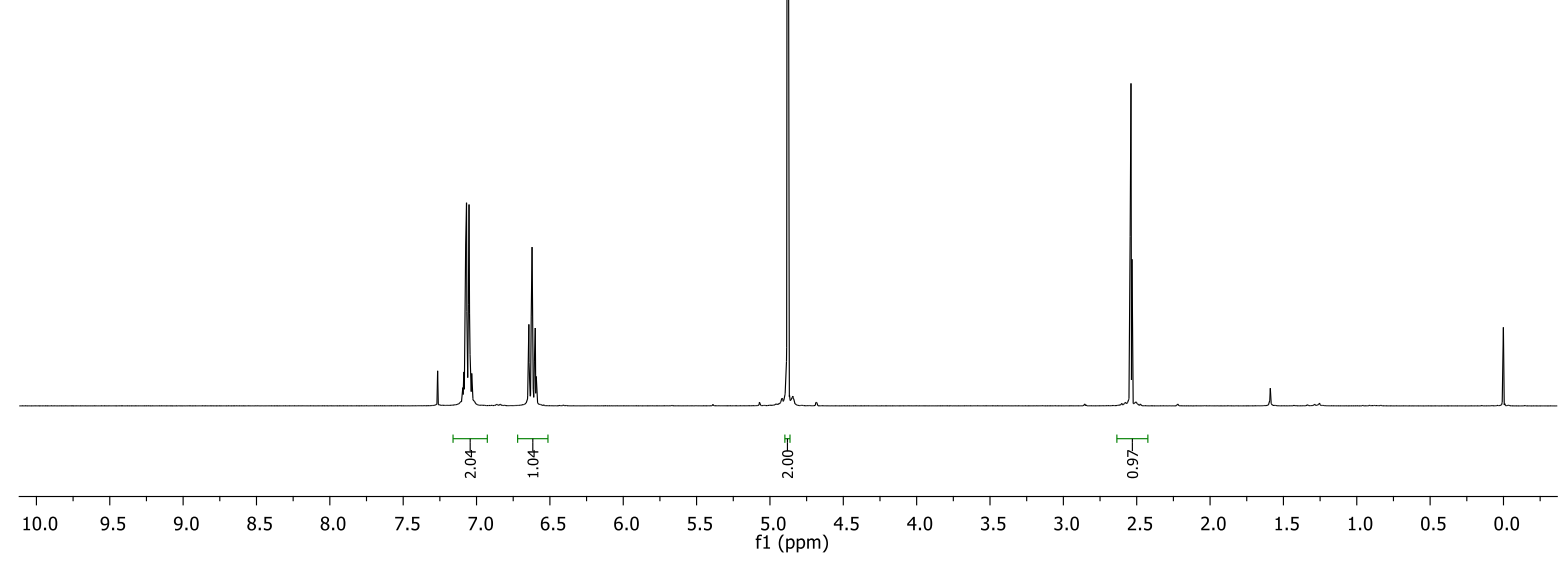<smiles>C#CCOC(=O)C(=N)c1cc(F)cc(F)c1</smiles>
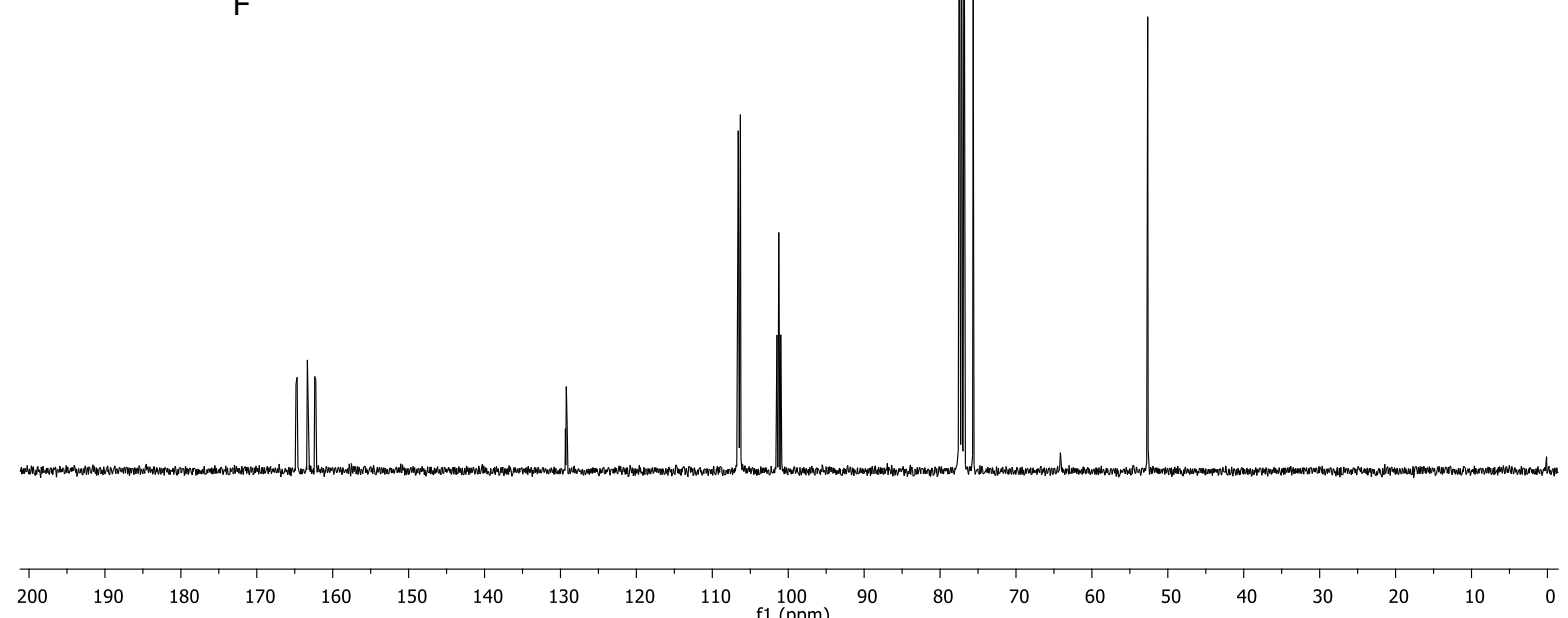

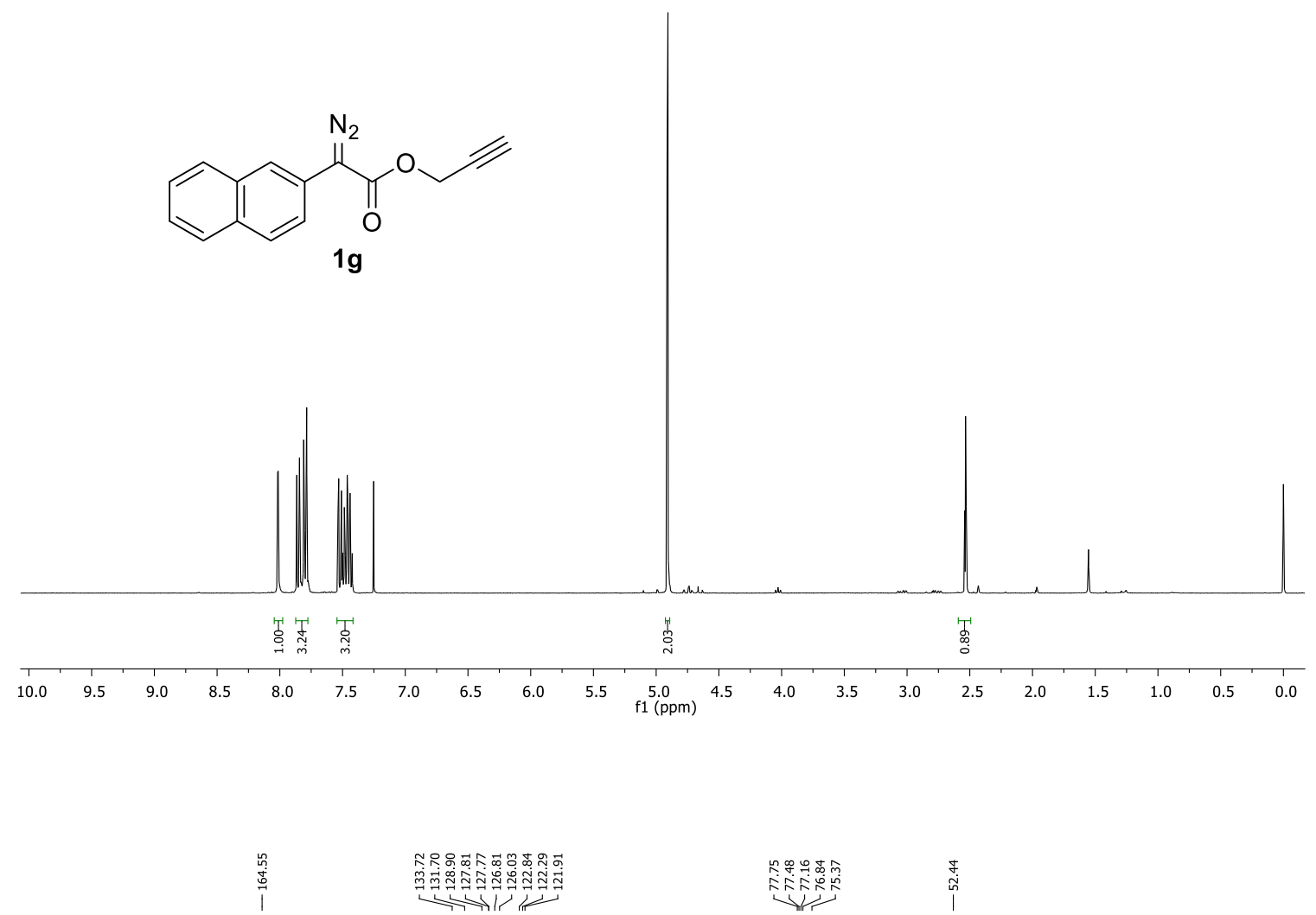

诲<smiles>C#CCOC(=O)C(=N)c1ccc2ccccc2c1</smiles>

$1 \mathrm{~g}$

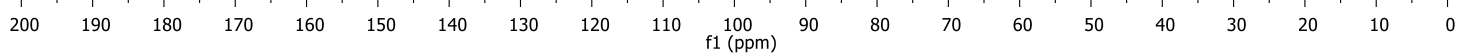




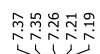
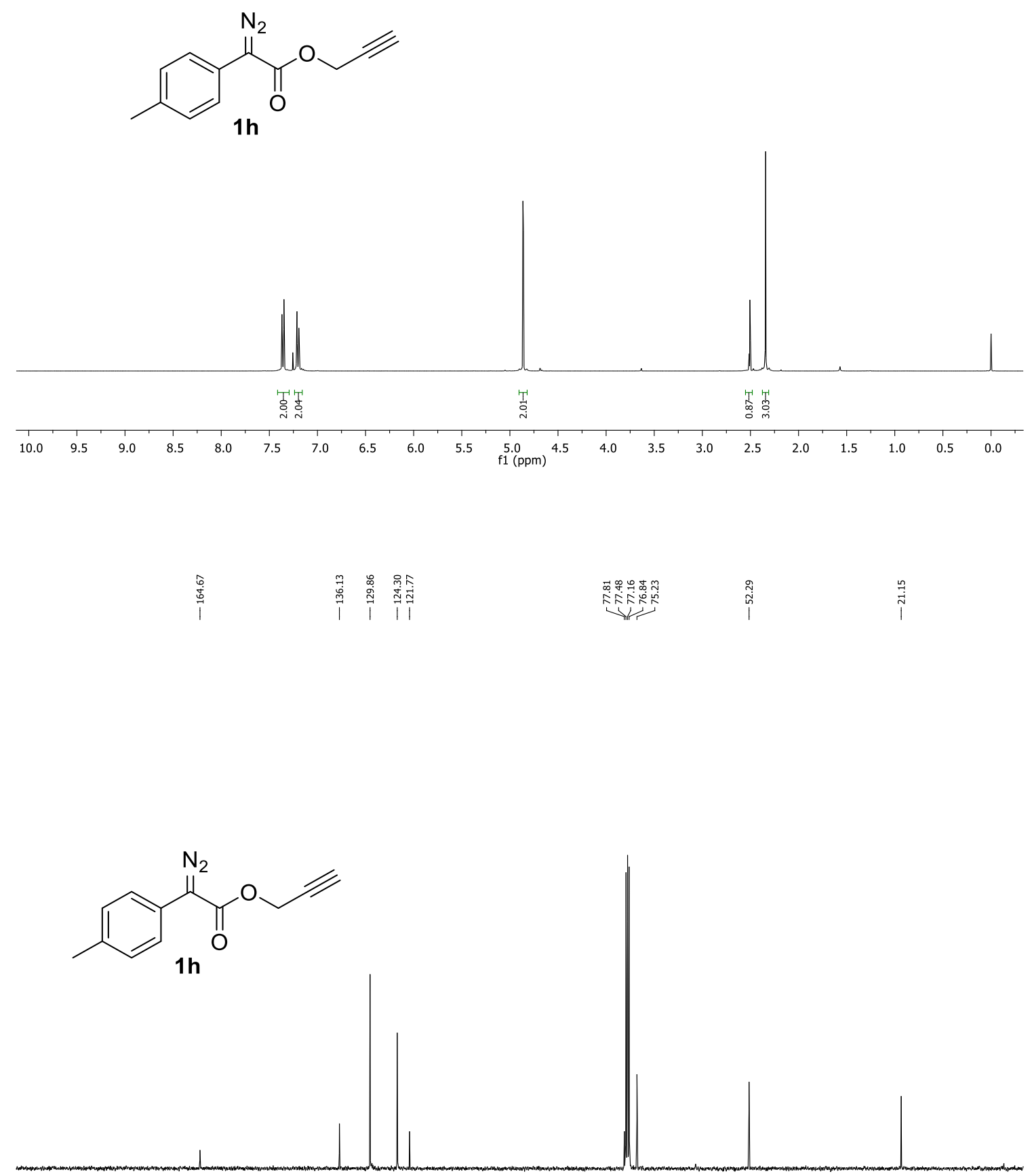

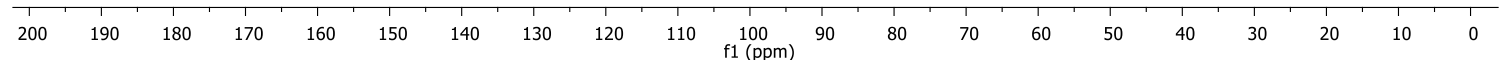




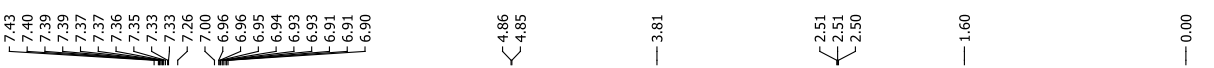

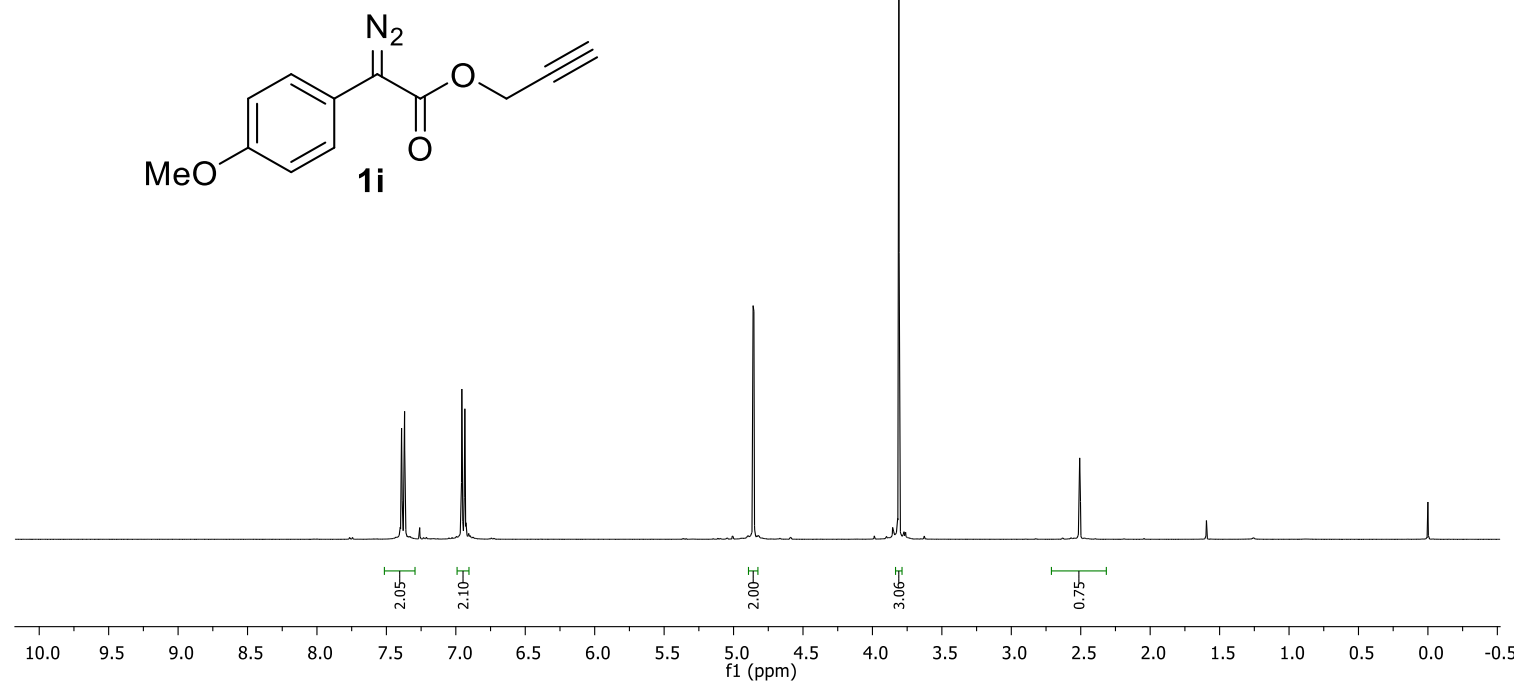

0
0

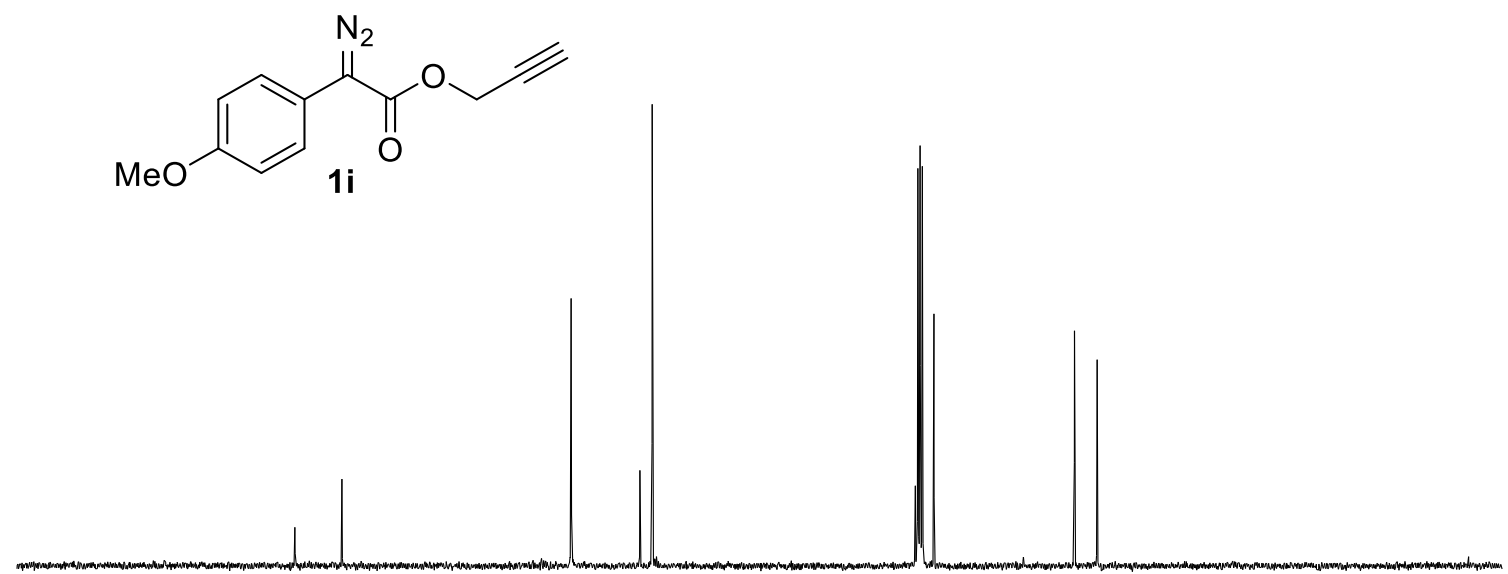

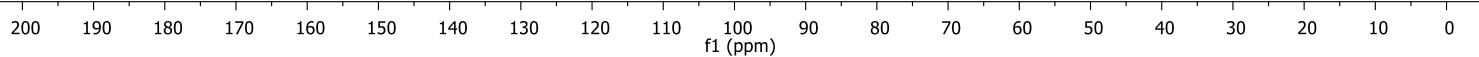




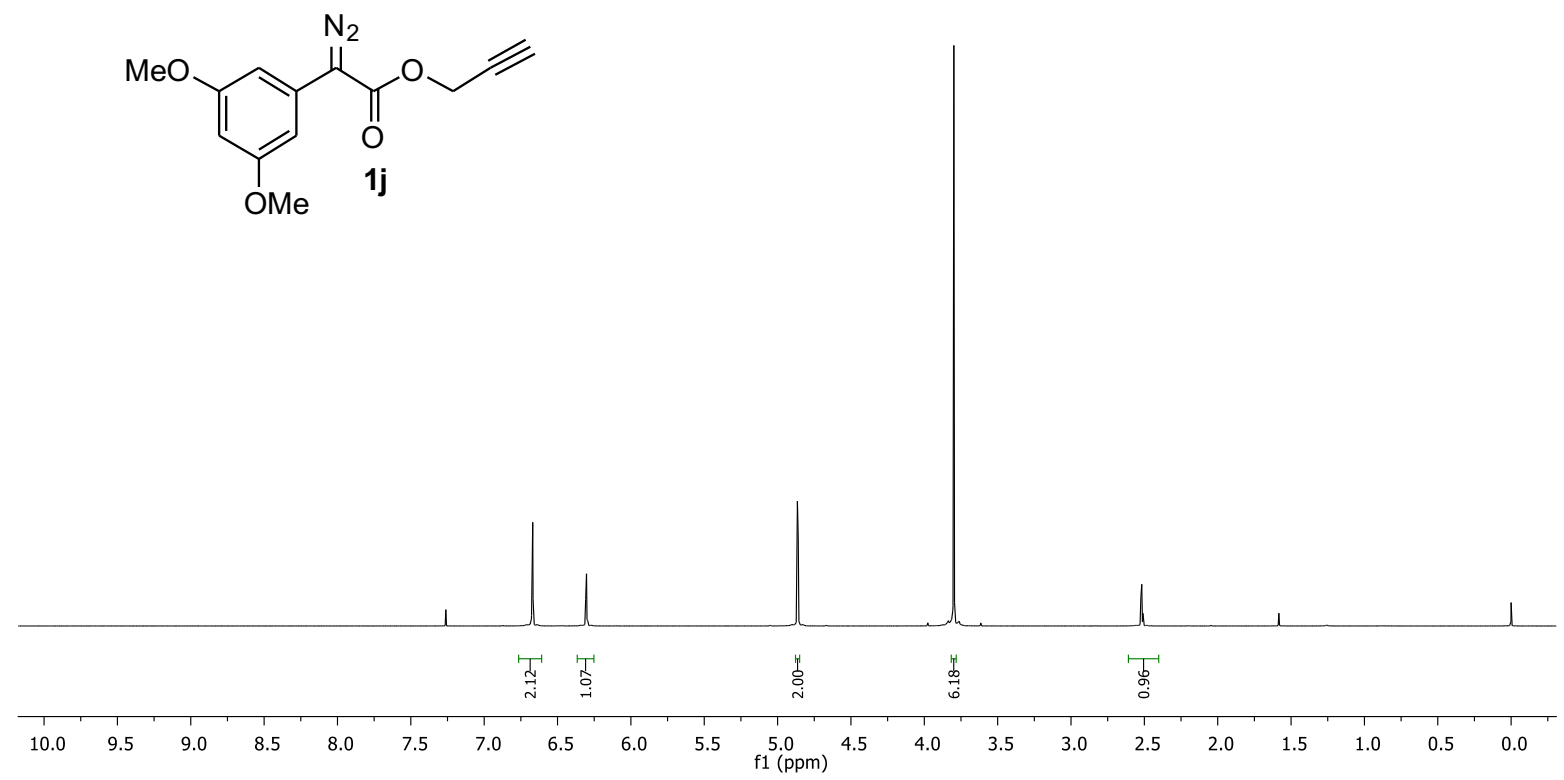




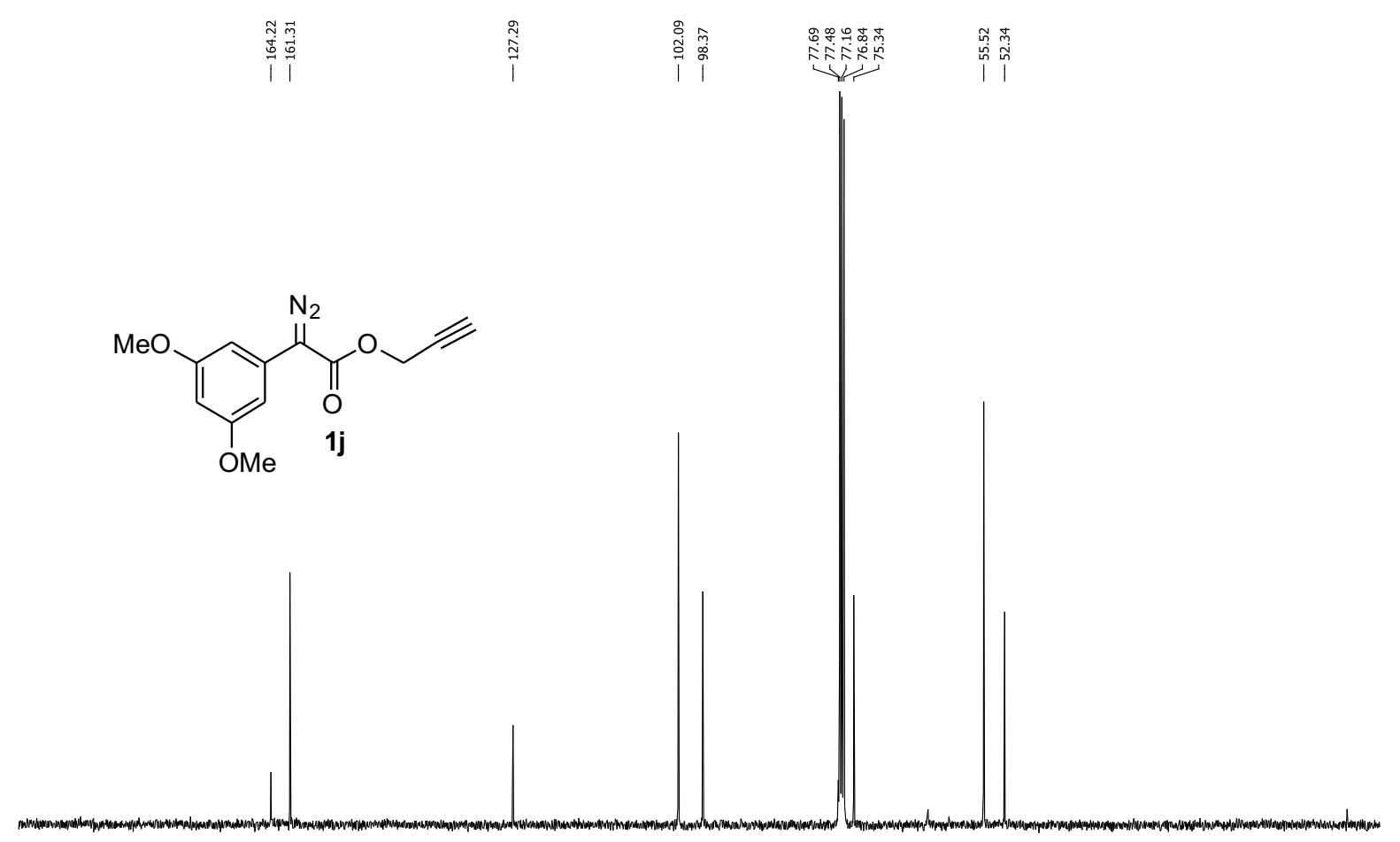

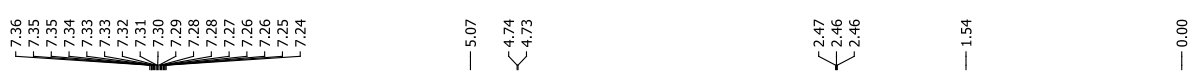

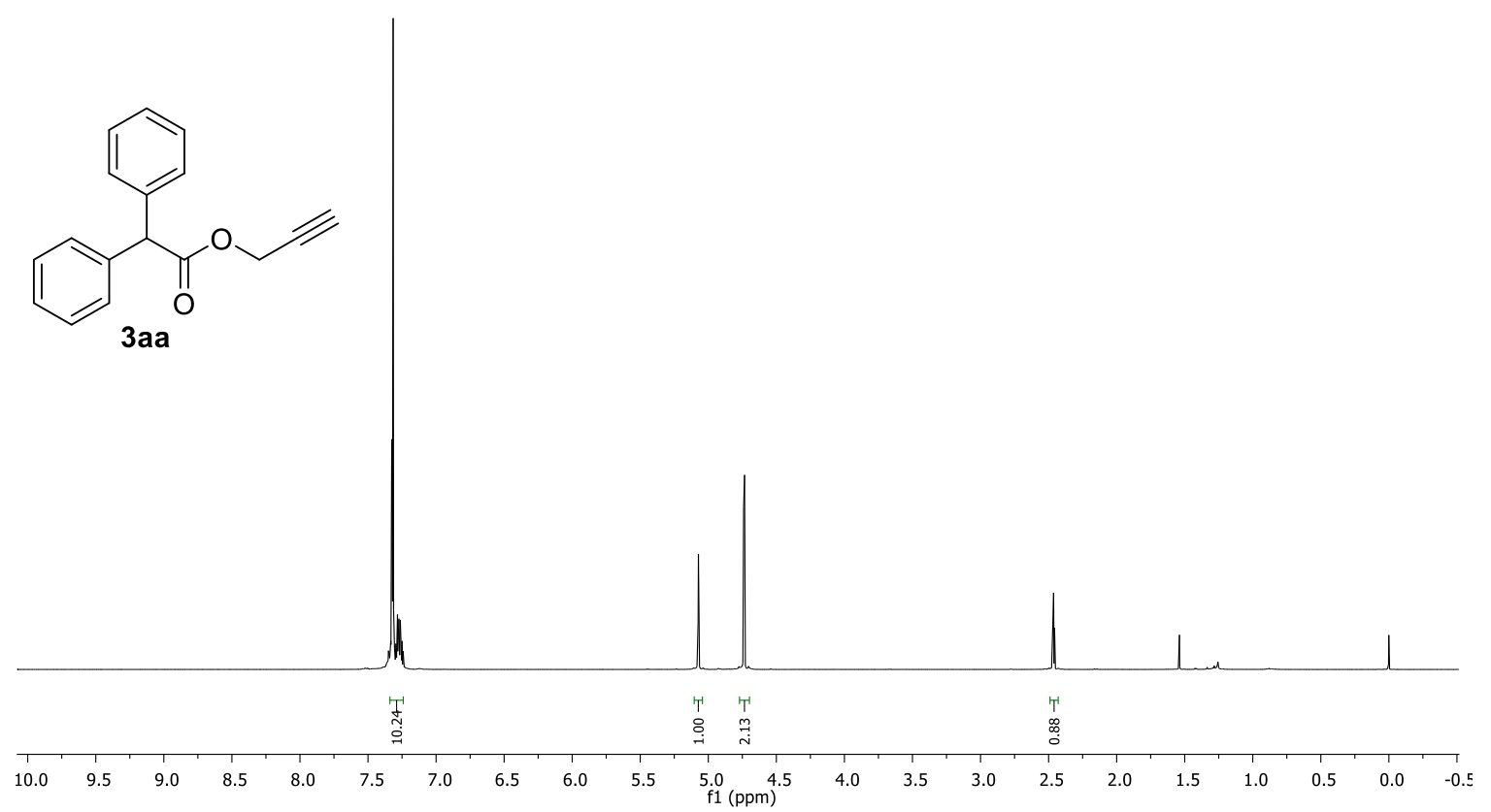




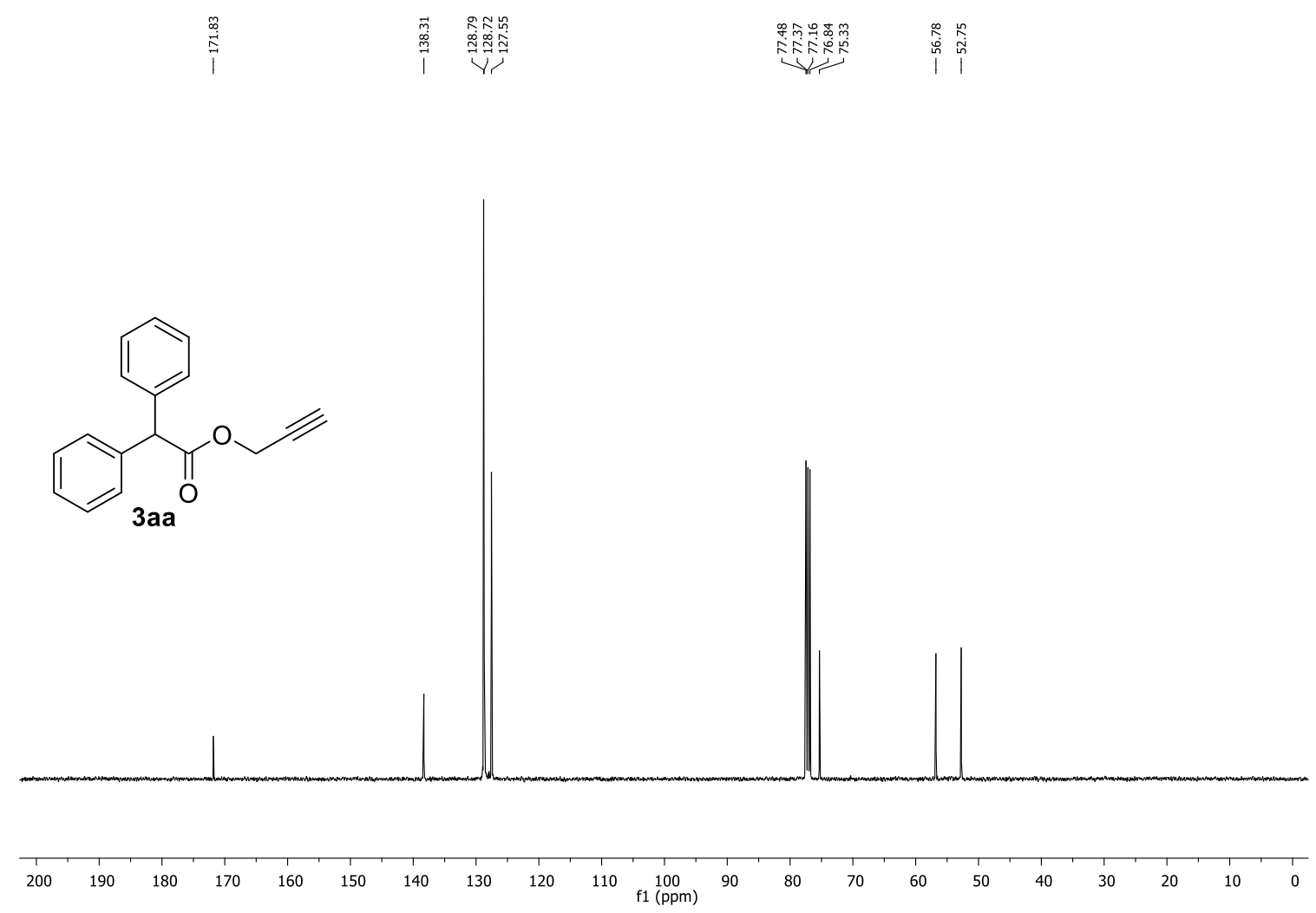

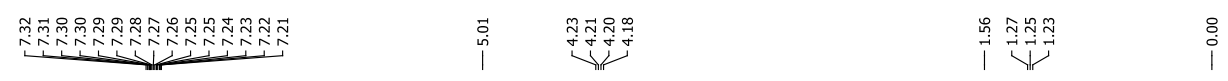

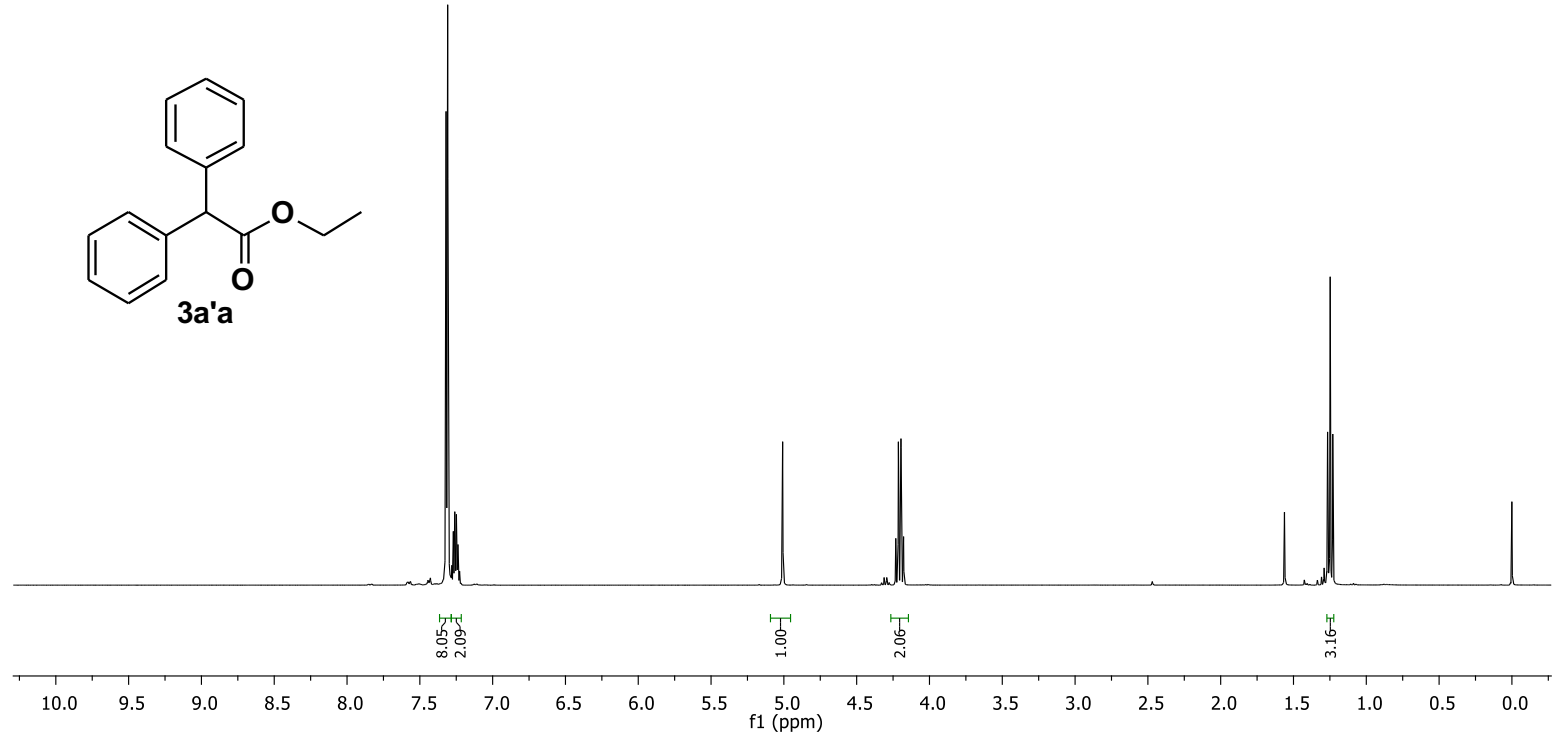


$\stackrel{\overrightarrow{0}}{\stackrel{0}{i}}$

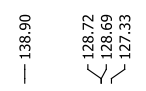

成

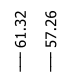

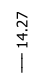
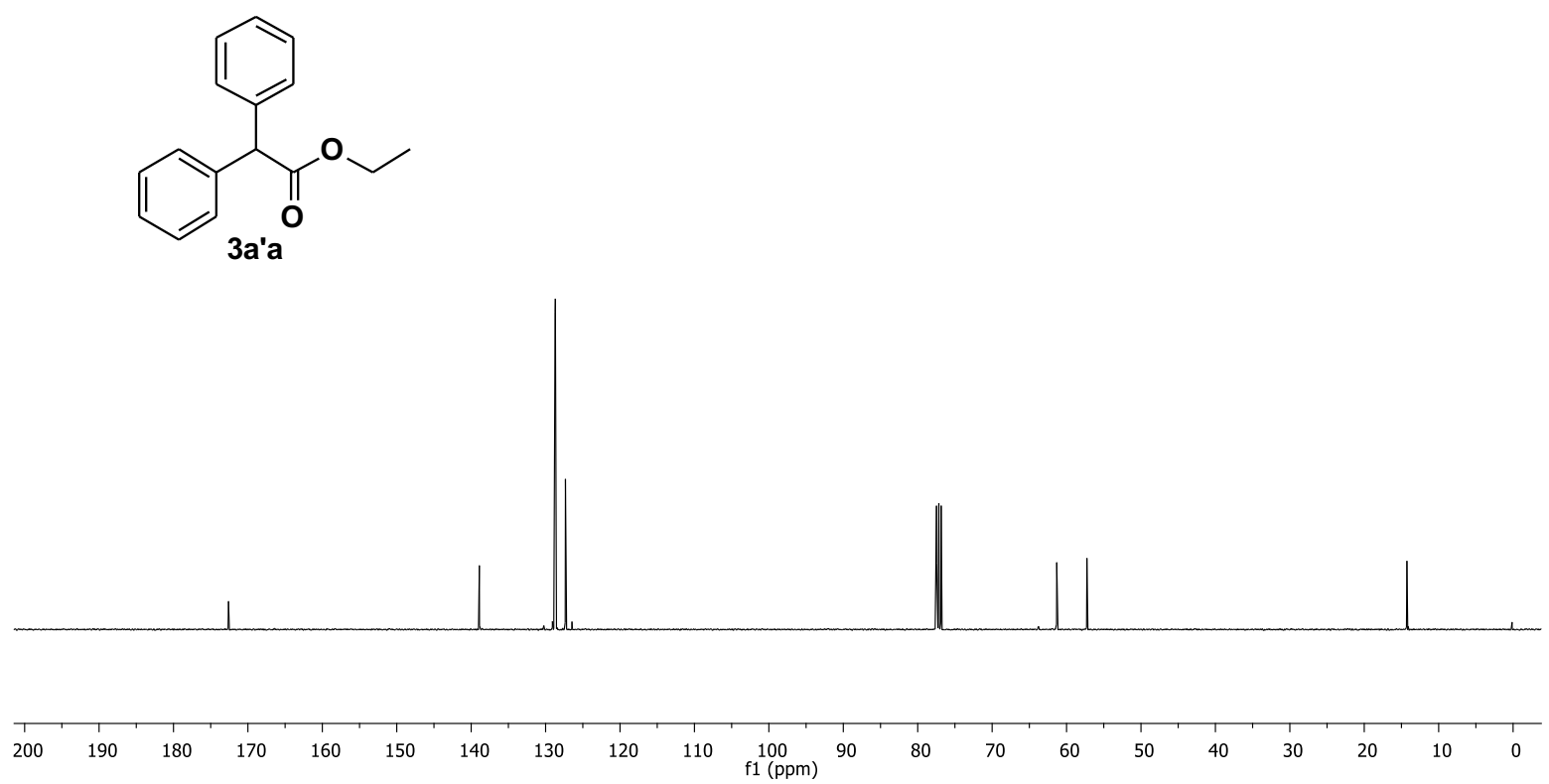

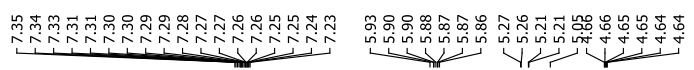

|

$\stackrel{8}{\circ}$
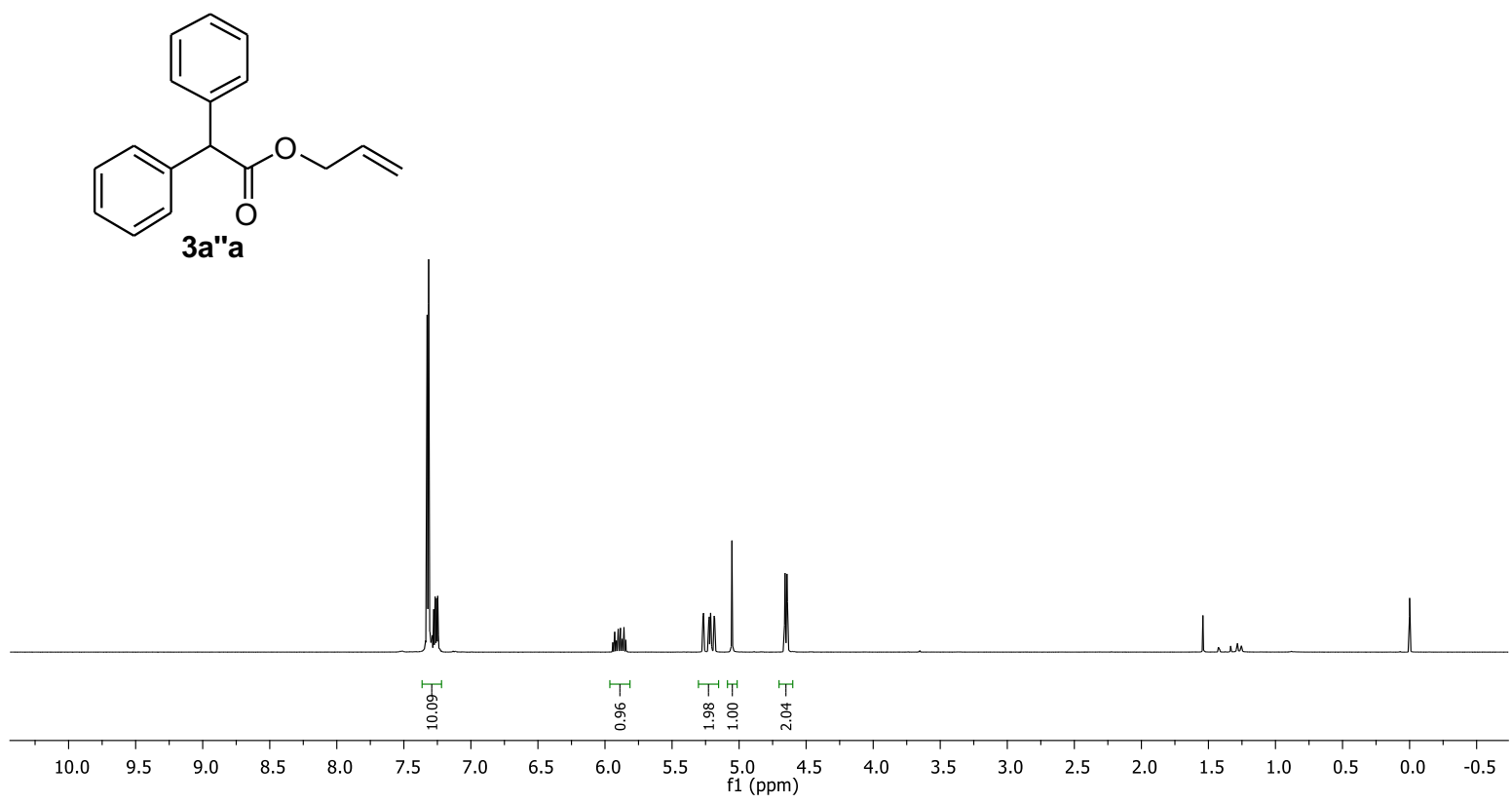

S77 


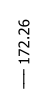

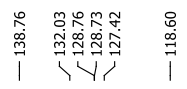

供 |
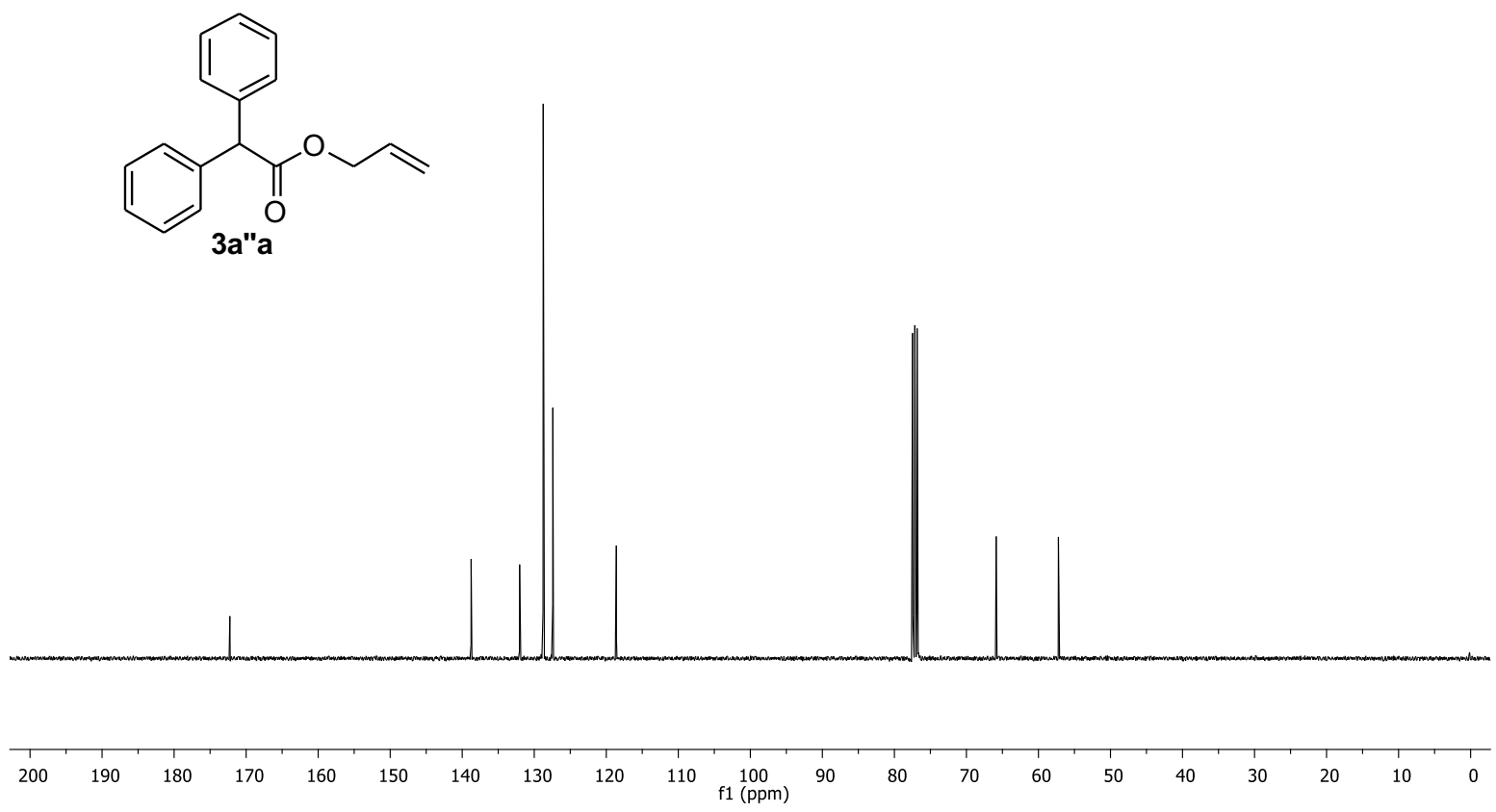

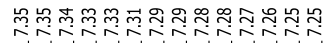

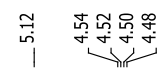

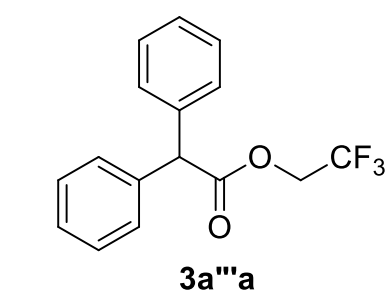

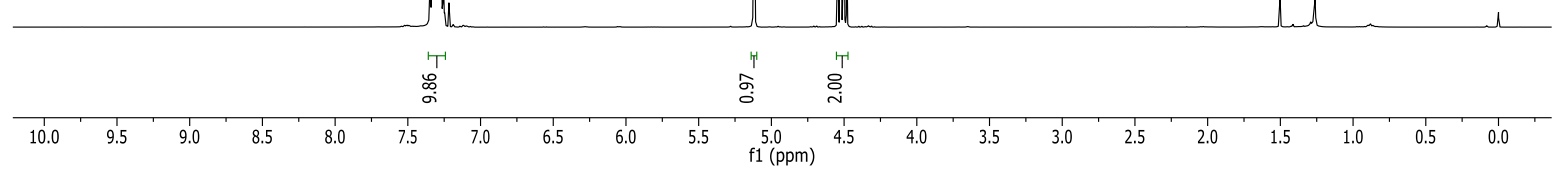

S78 

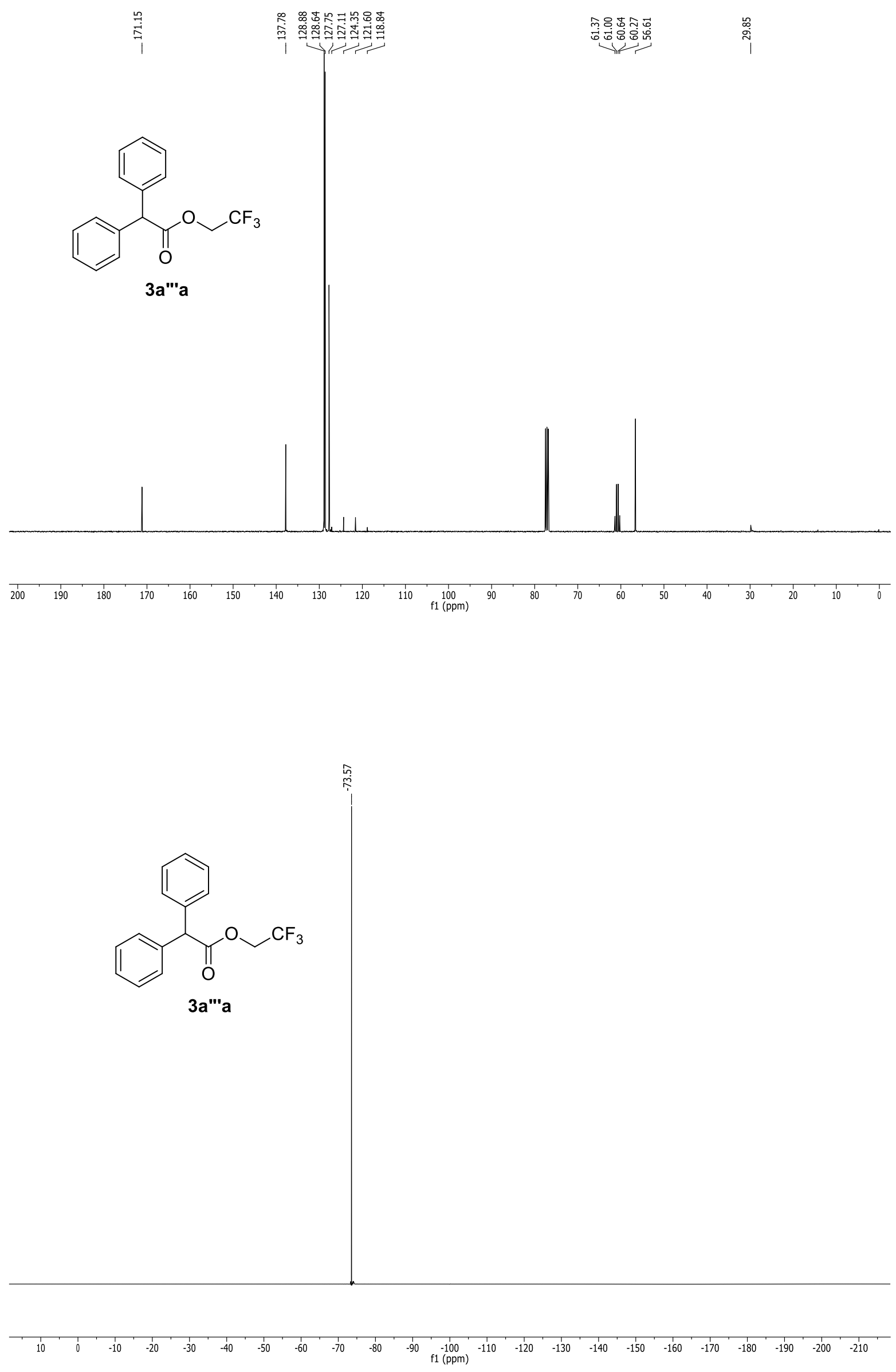

S79 
<smiles>C#CCOC(=O)C(c1ccccc1)c1ccc(Br)cc1</smiles>

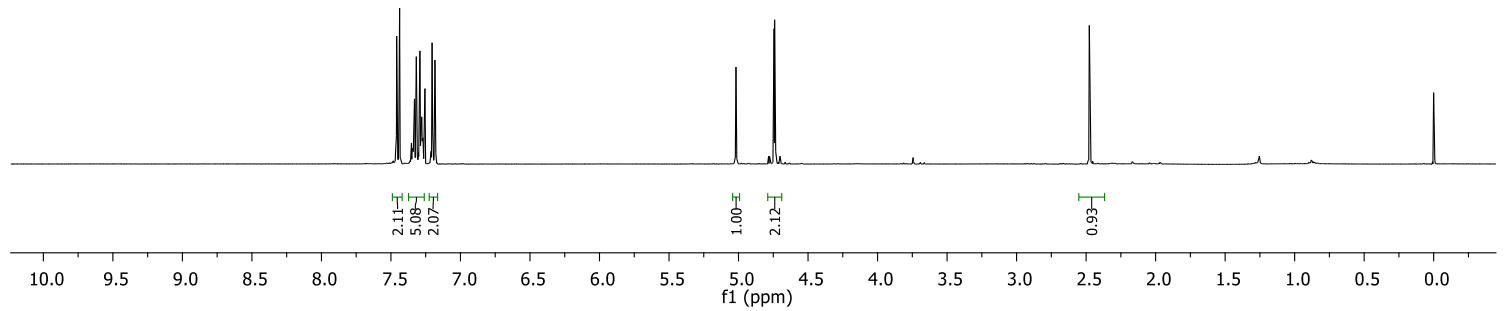



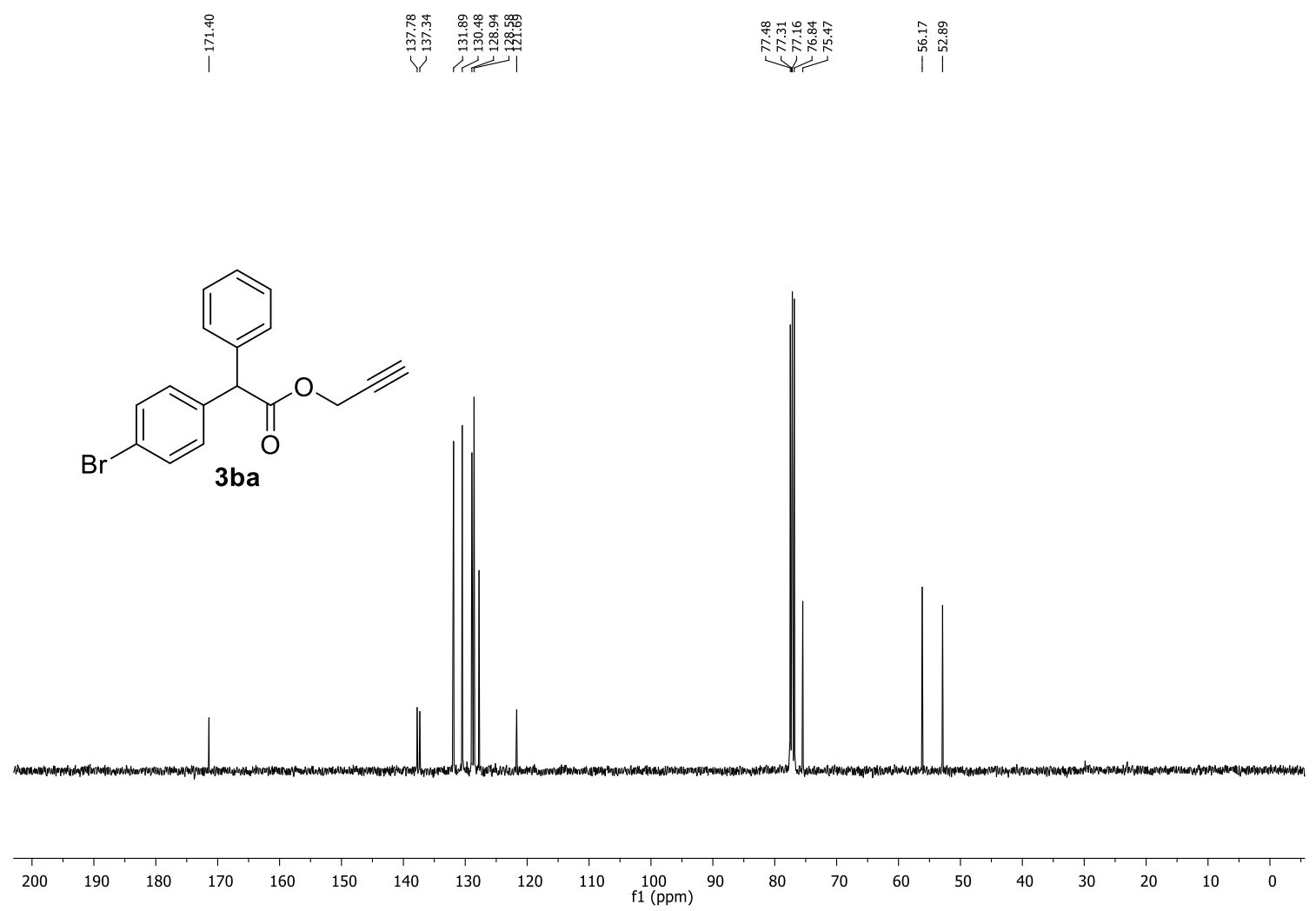

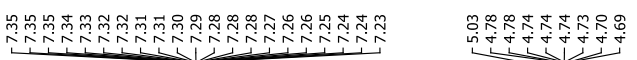

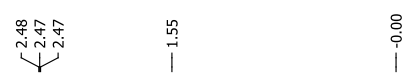<smiles>C#CCOC(=O)C(c1ccccc1)c1ccc(Cl)cc1</smiles>

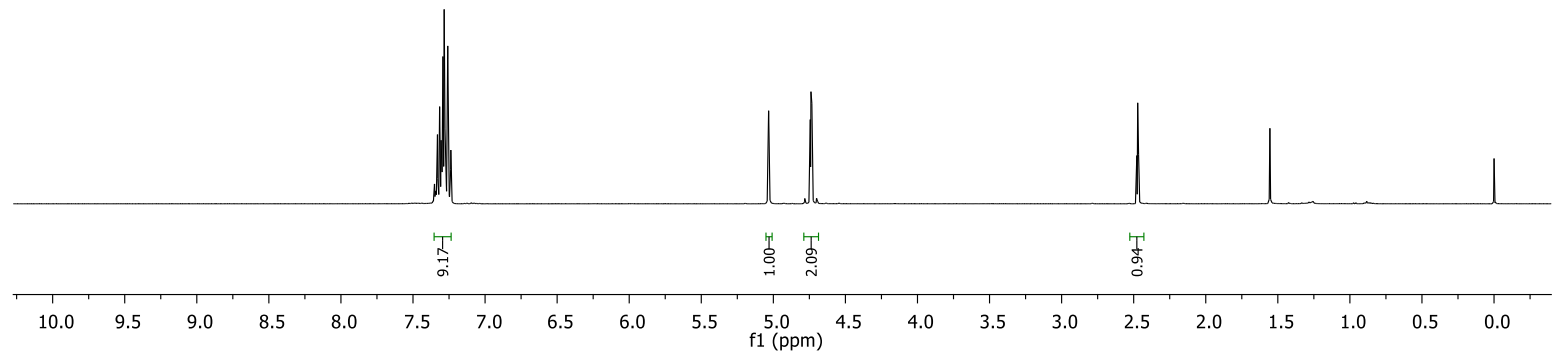




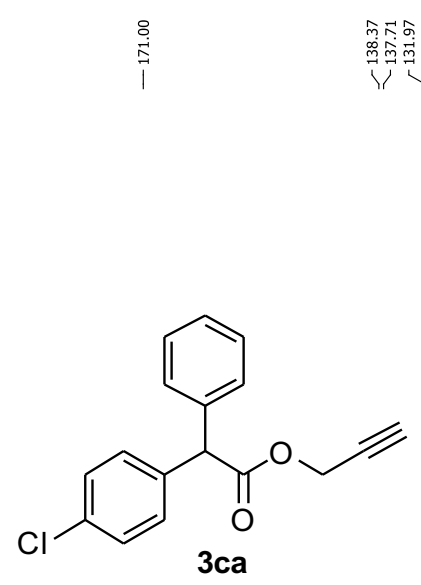

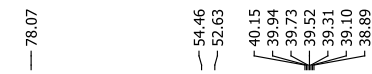

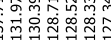

(1)

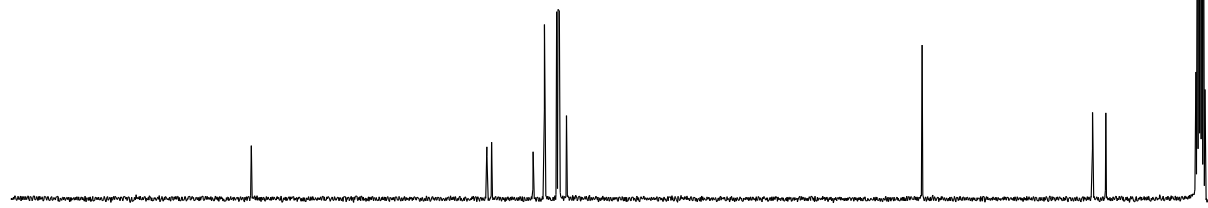

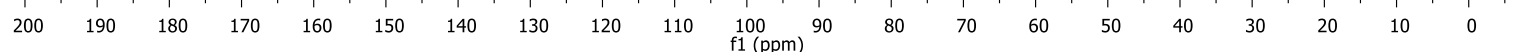

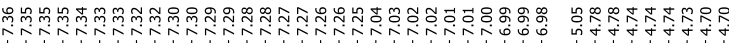

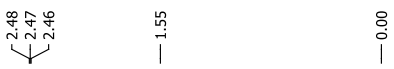<smiles>C#CCOC(=O)C(c1ccccc1)c1ccc(F)cc1</smiles>

3da

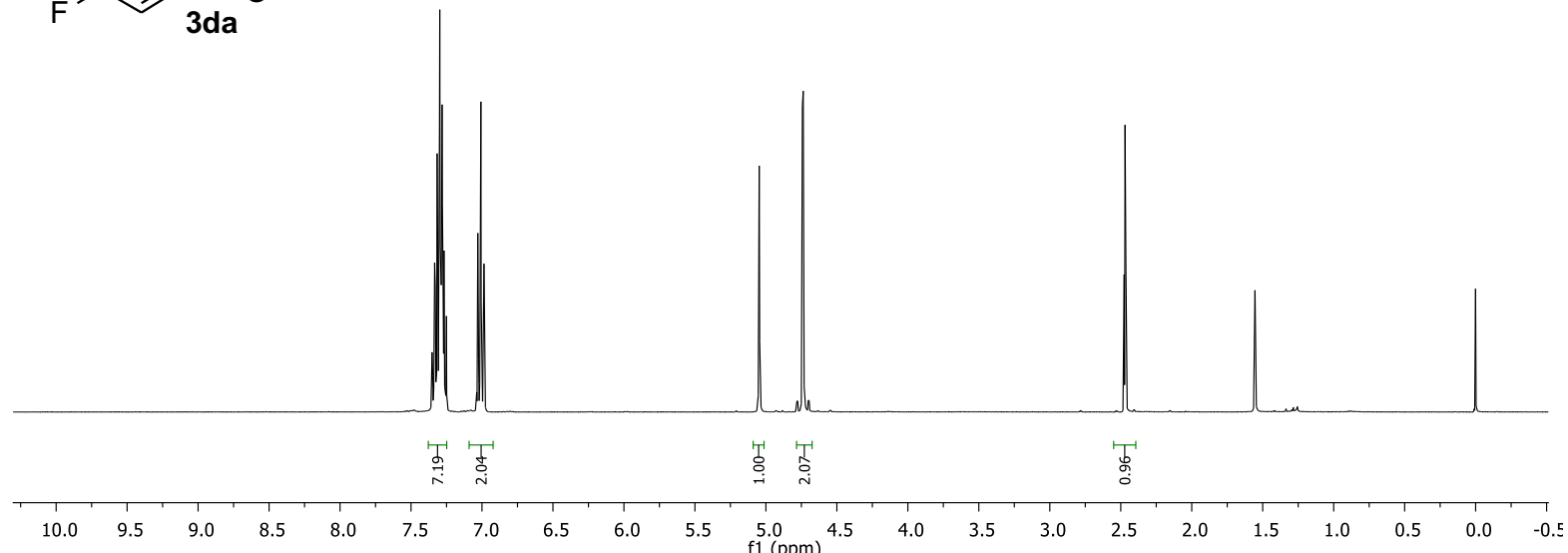




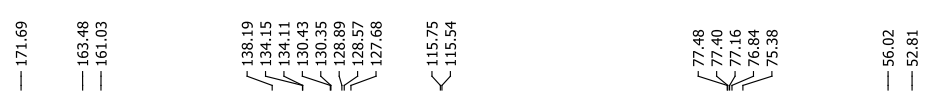
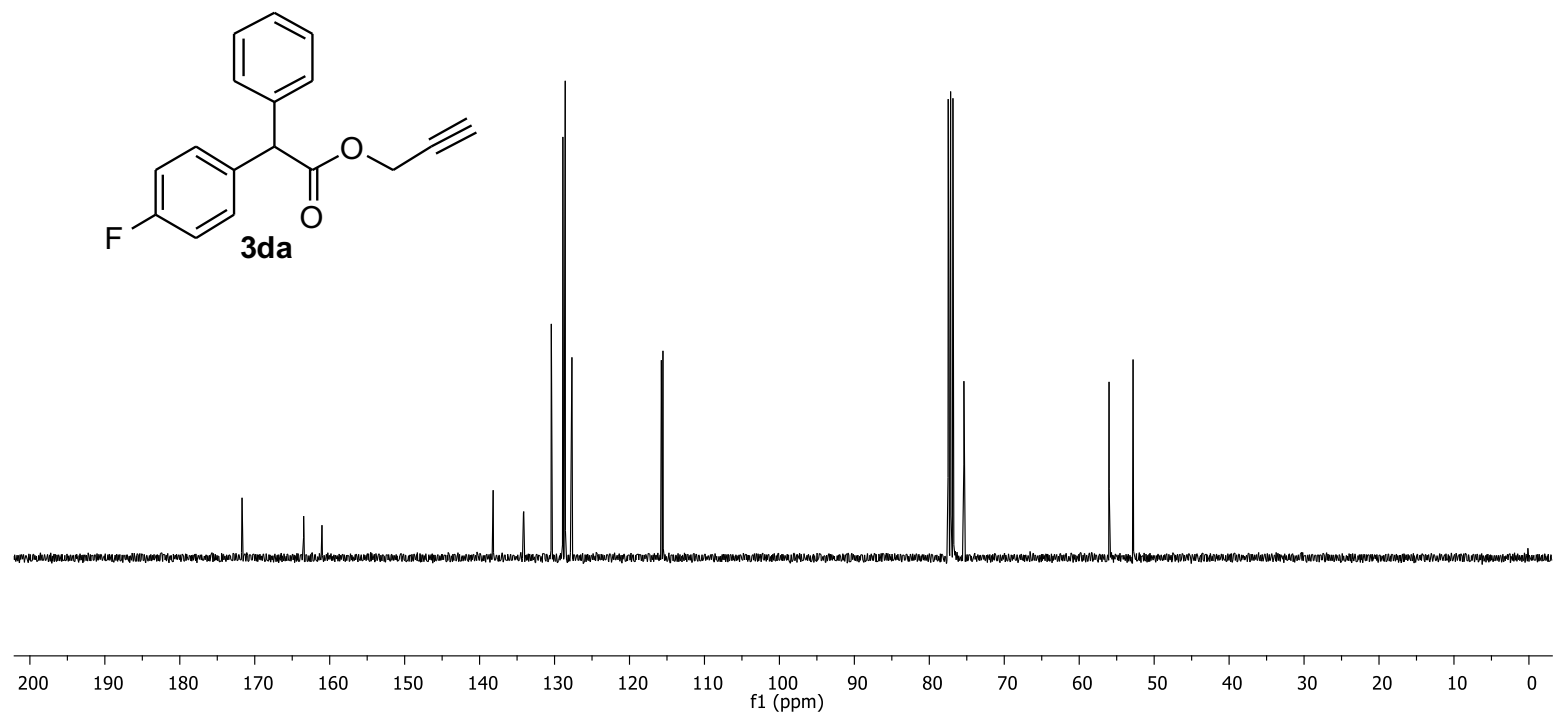

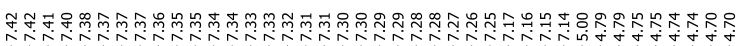

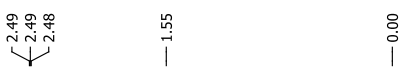

(clea

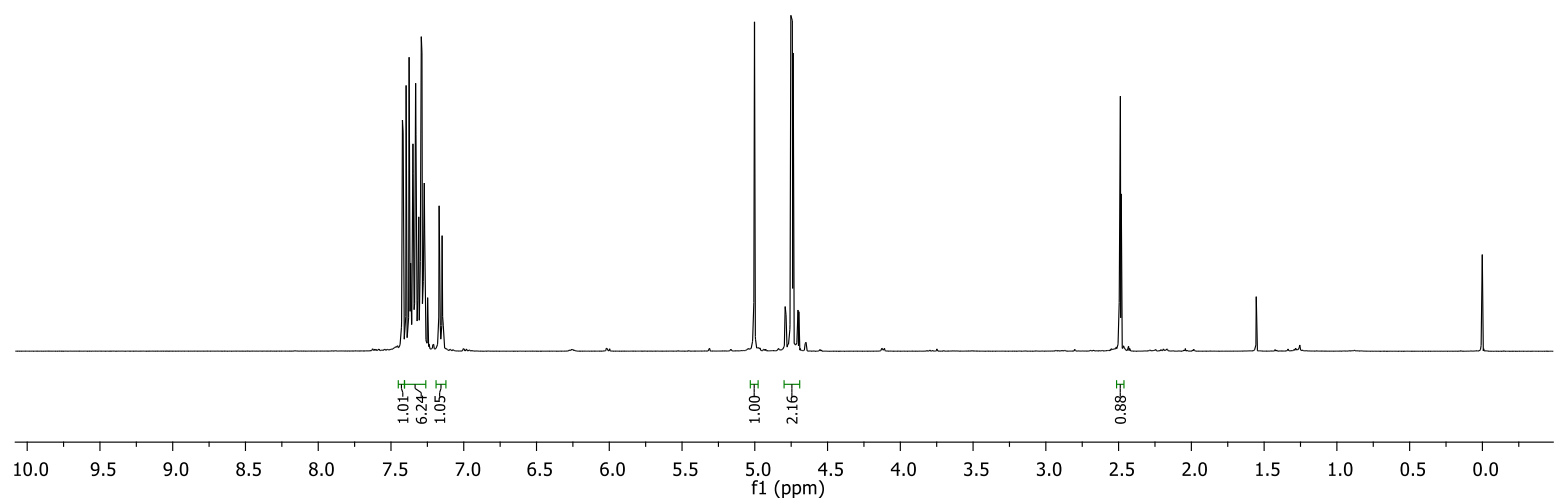



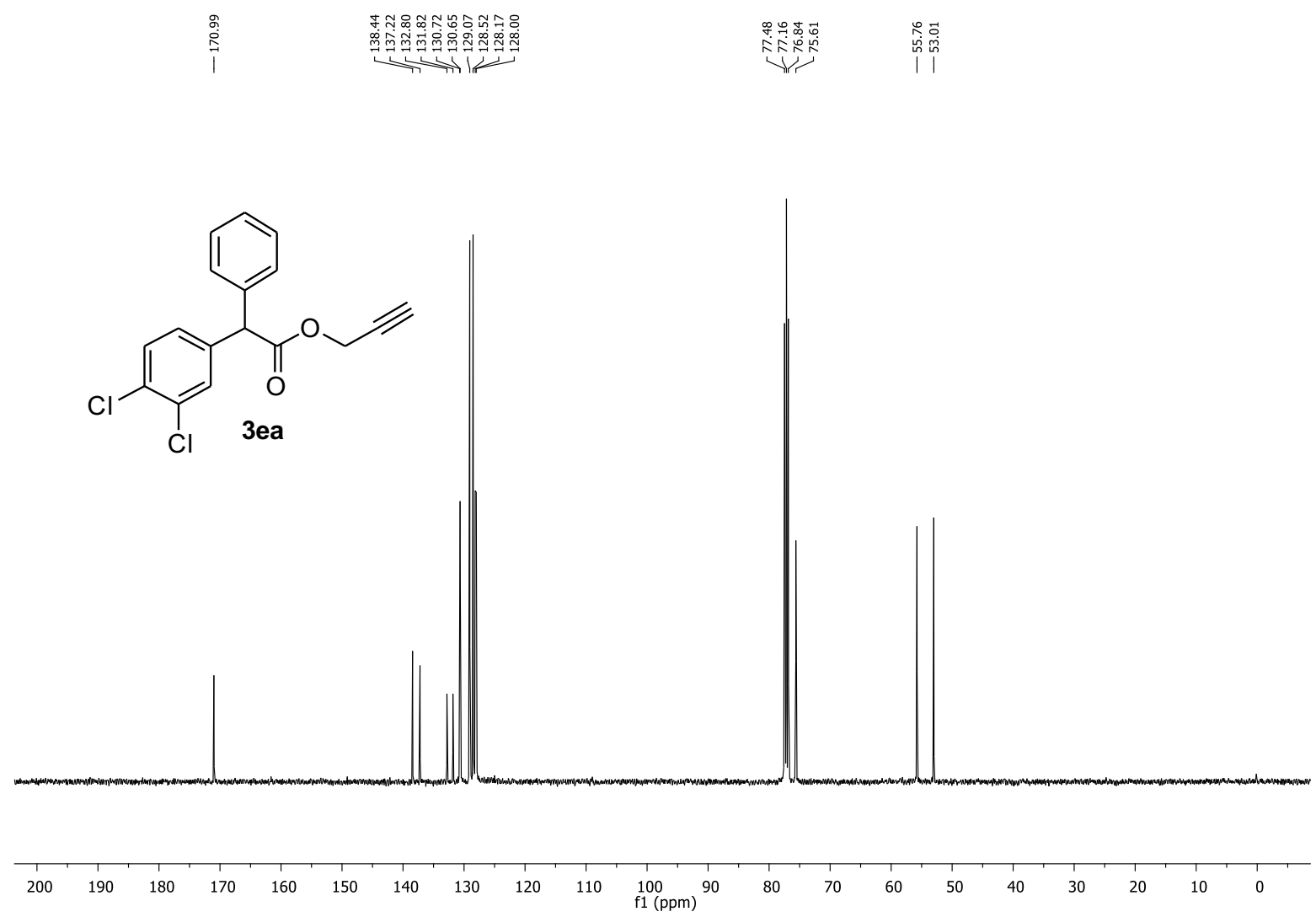

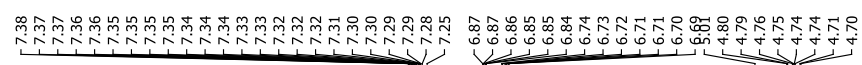<smiles>C#CCOC(=O)C(c1ccccc1)c1cc(F)cc(F)c1</smiles>

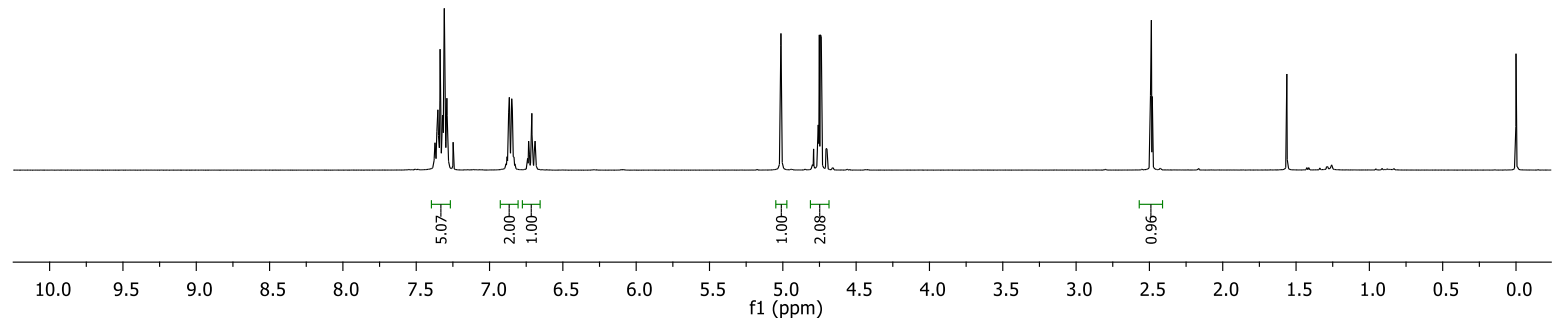




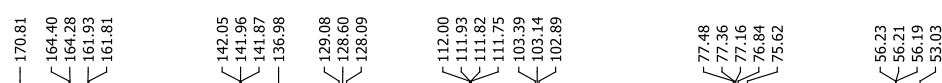

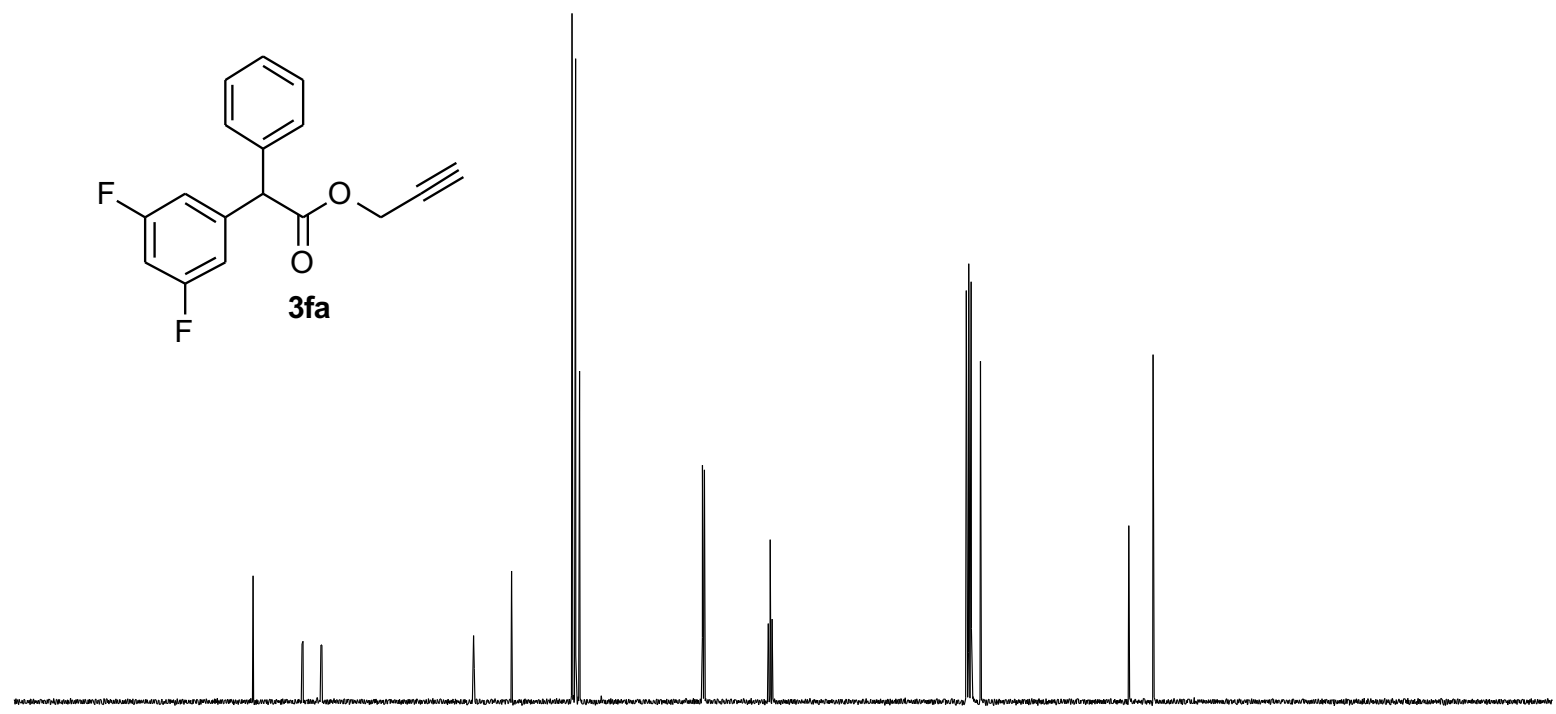

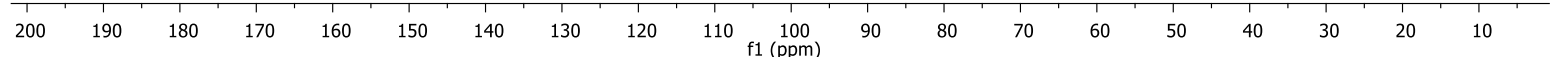

\begin{tabular}{|c|c|}
\hline 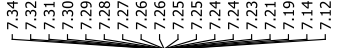 & 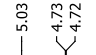 \\
\hline
\end{tabular}<smiles>C#CCOC(=O)C(c1ccccc1)c1ccc(C)cc1</smiles>

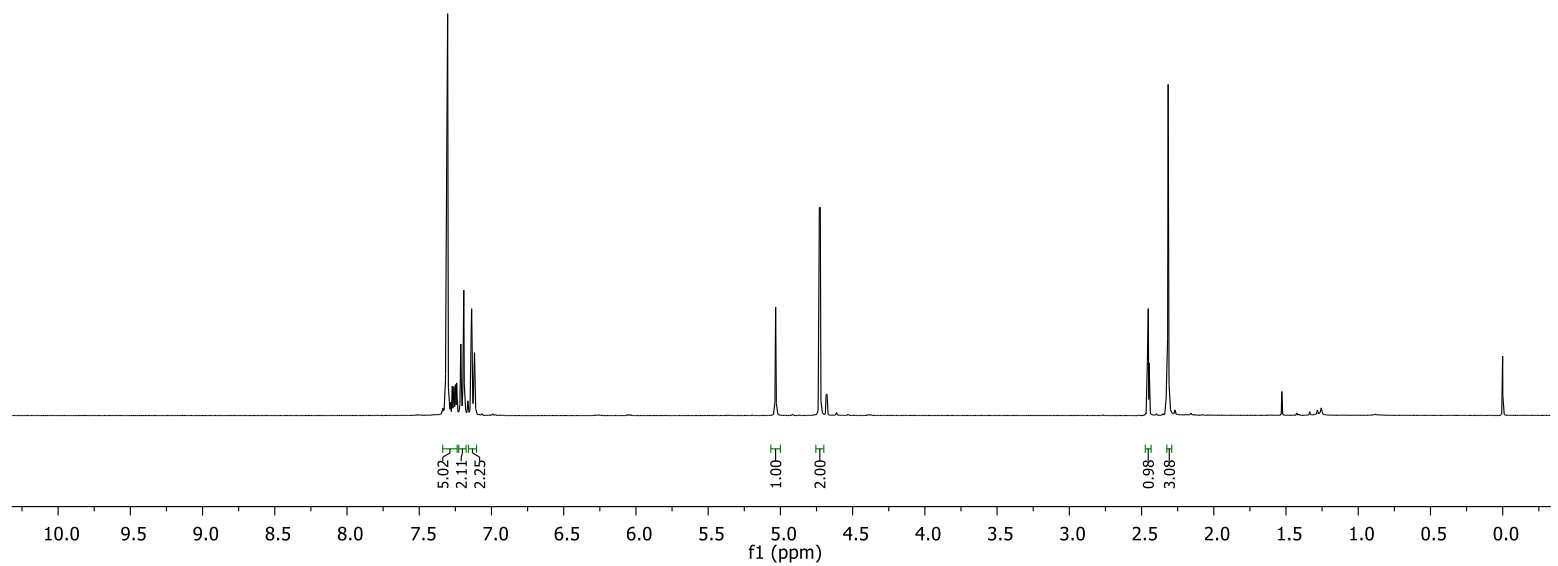


<smiles>C#CCOC(=O)C(c1ccccc1)c1ccc(C)cc1</smiles>

3ha
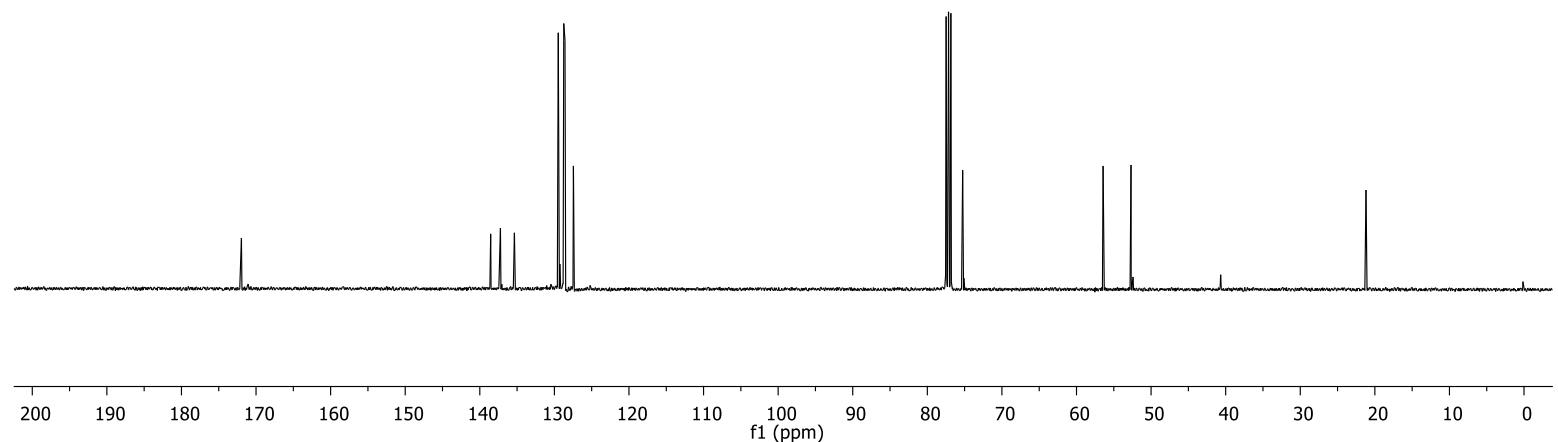

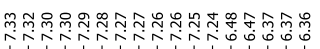

旅走寺

$\sqrt{\sqrt{2}}$<smiles>C#CCOC(=O)C(c1ccccc1)c1cc(OC)cc(OC)c1</smiles>

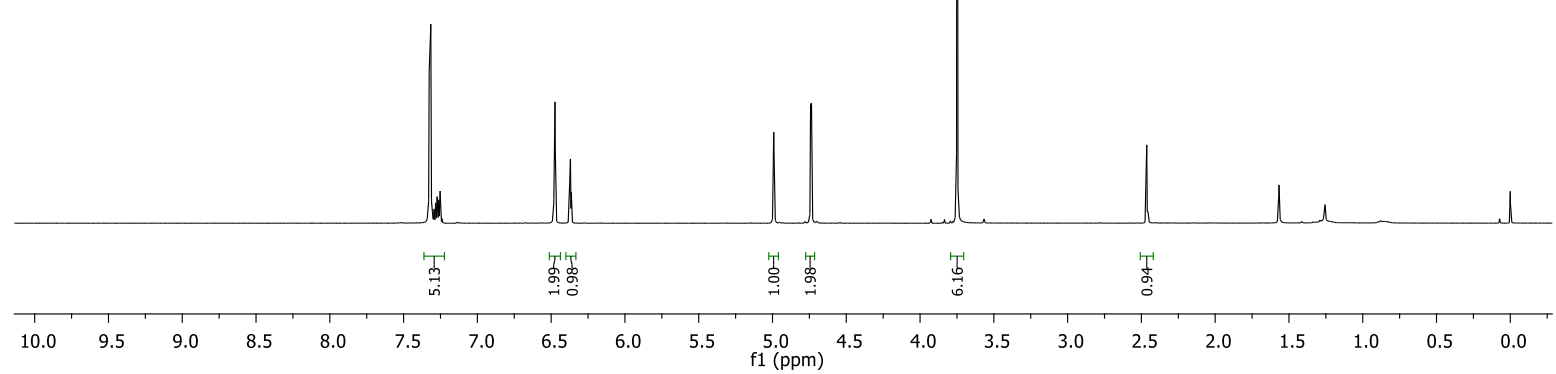


|
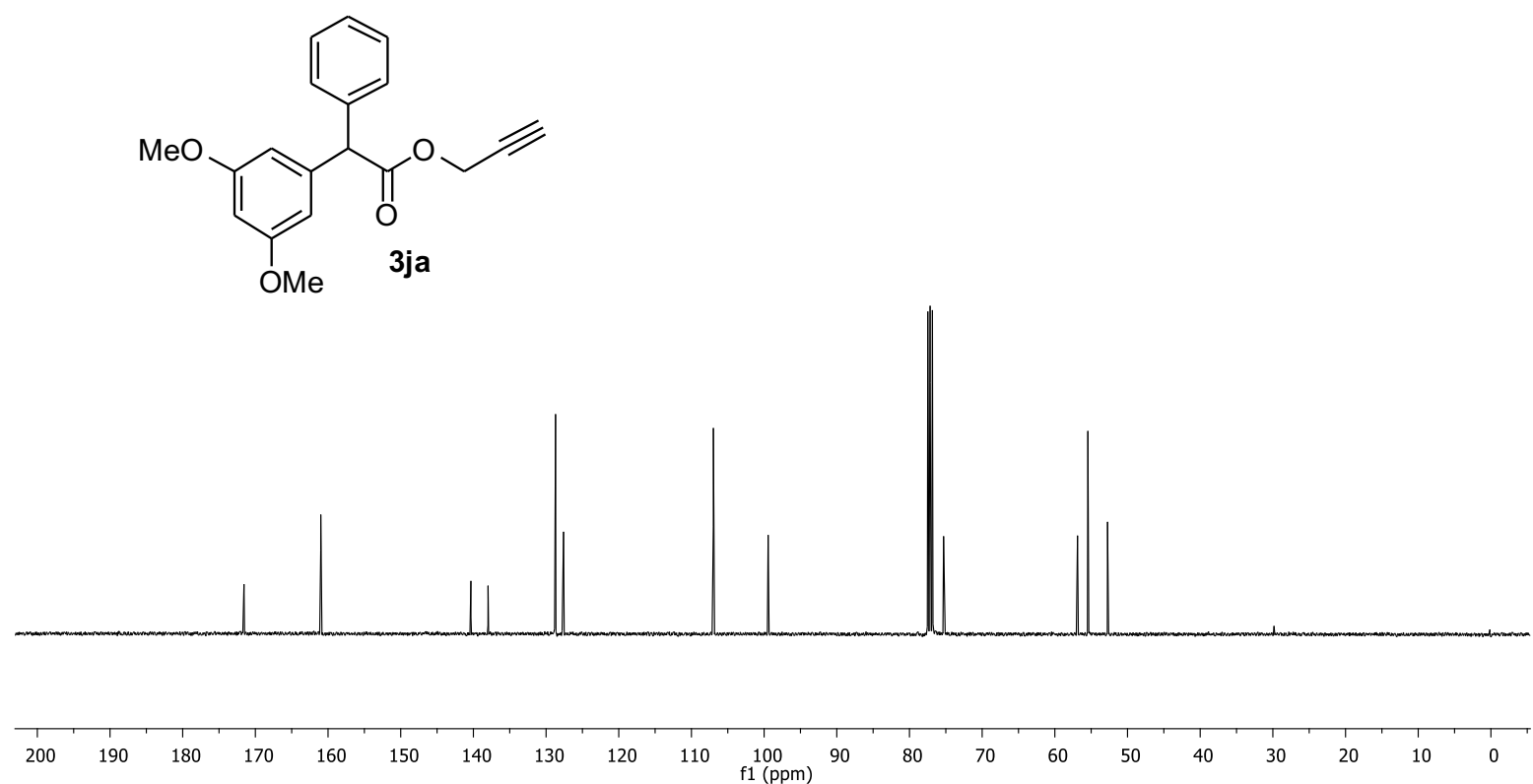

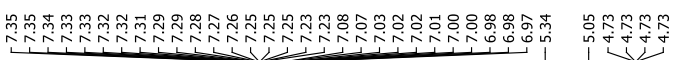
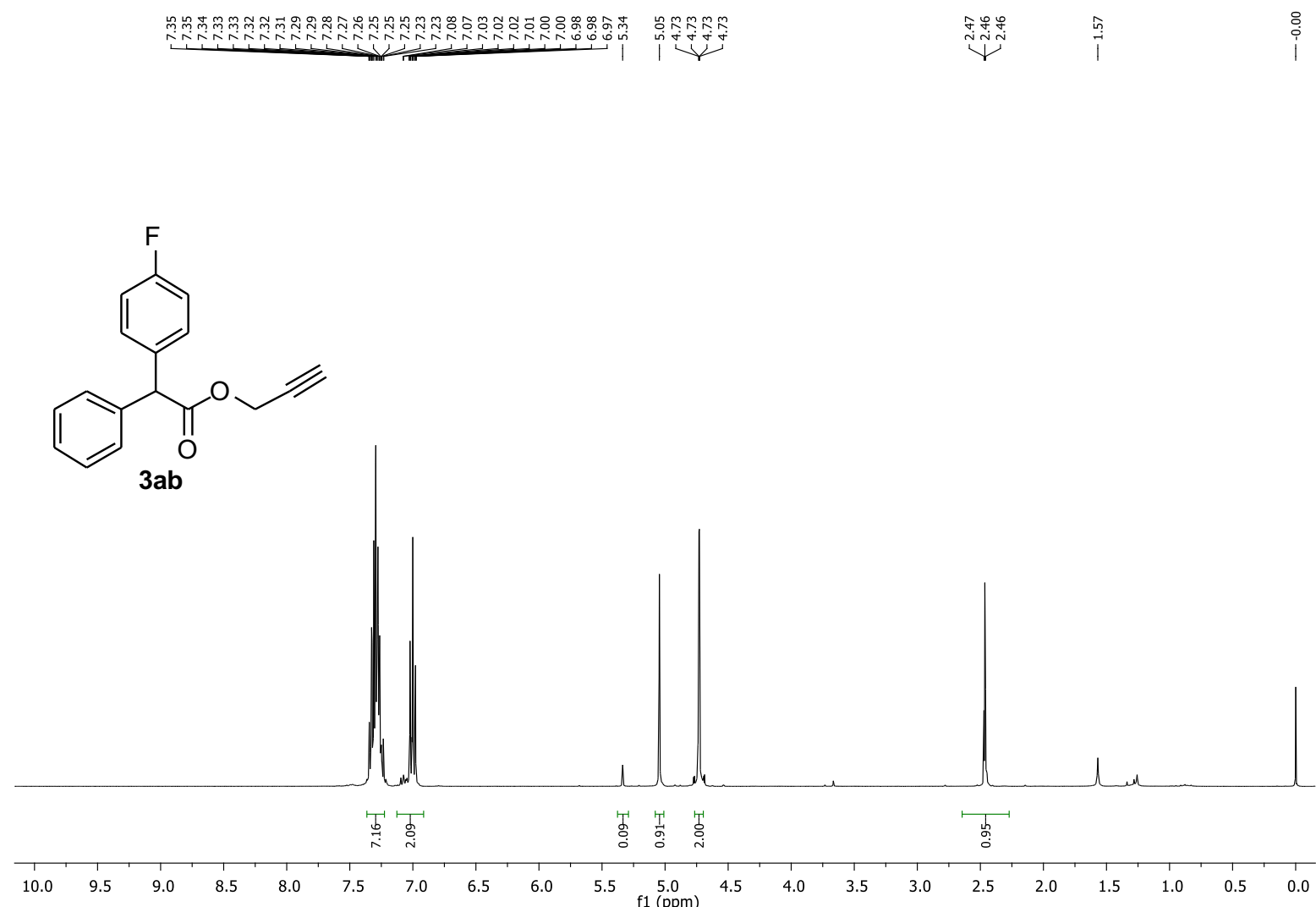


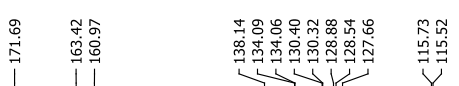

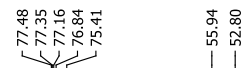<smiles>C#CCOC(=O)C(c1ccccc1)c1ccc(F)cc1</smiles>

3ab

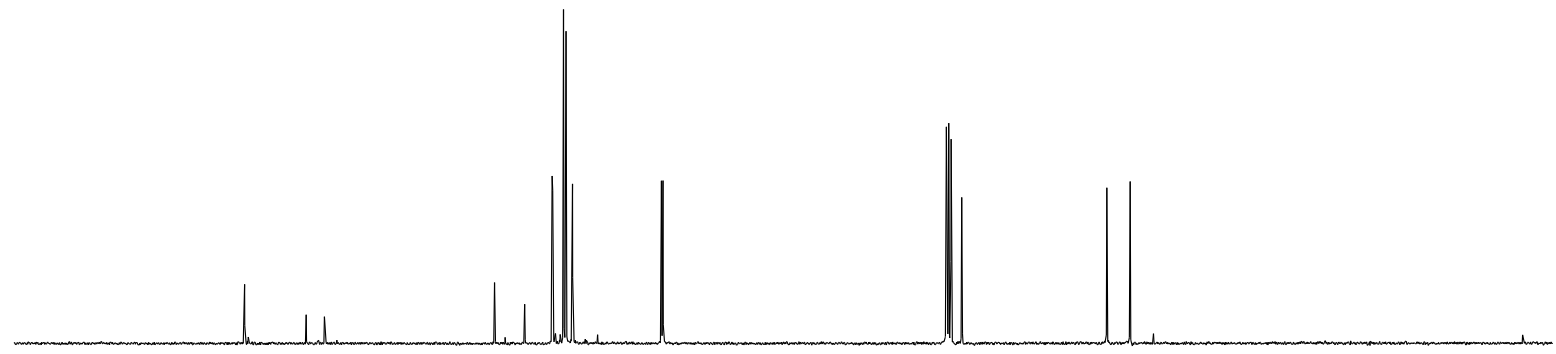

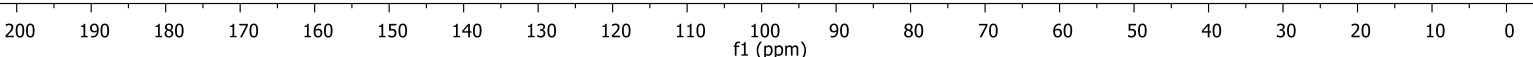

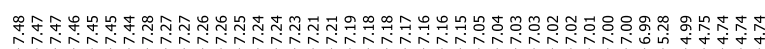

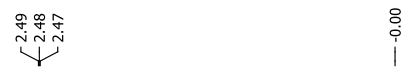

(c)

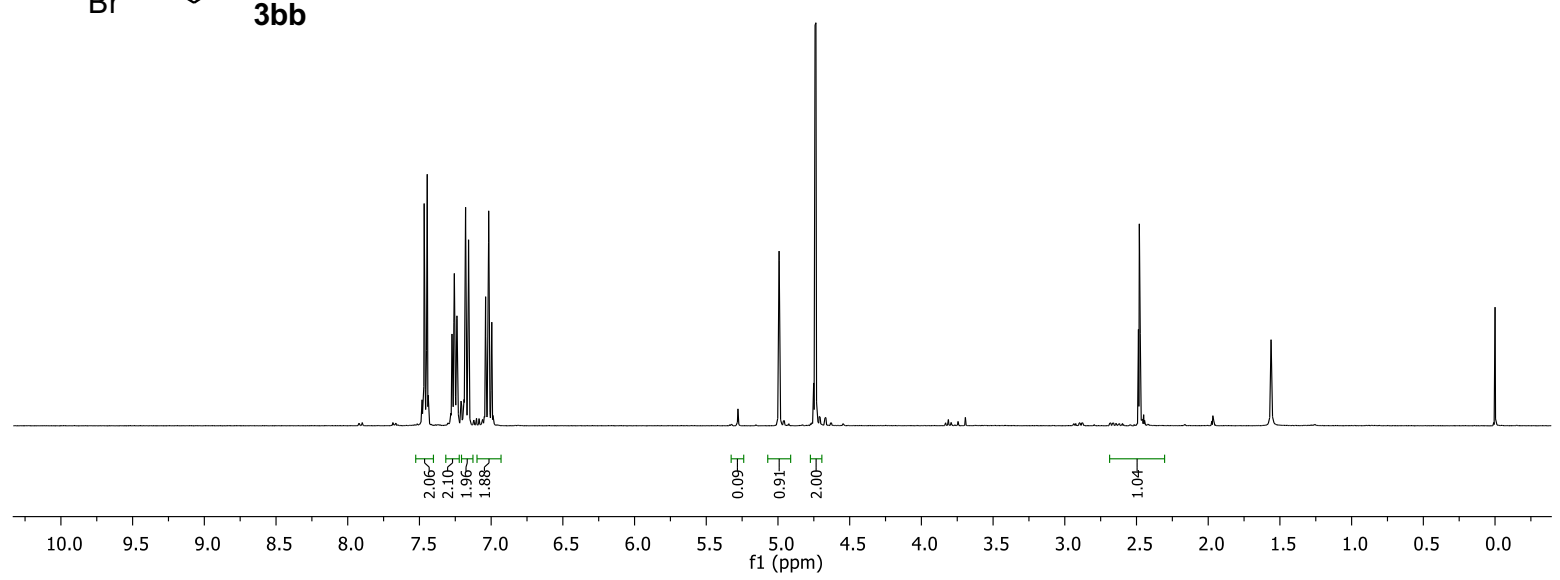




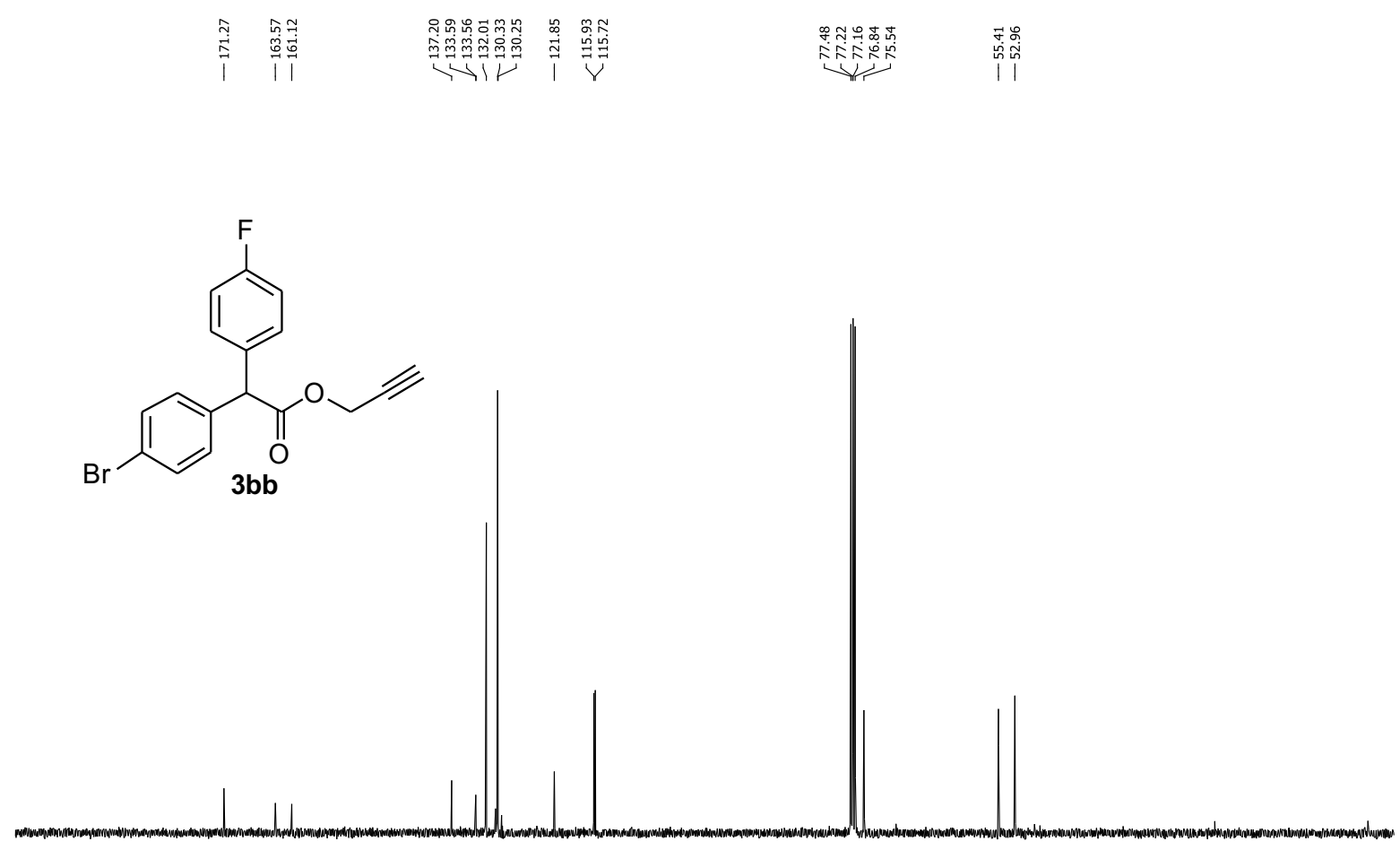

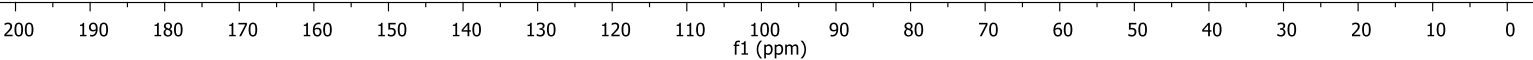

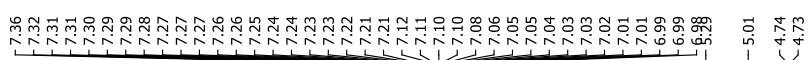

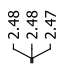

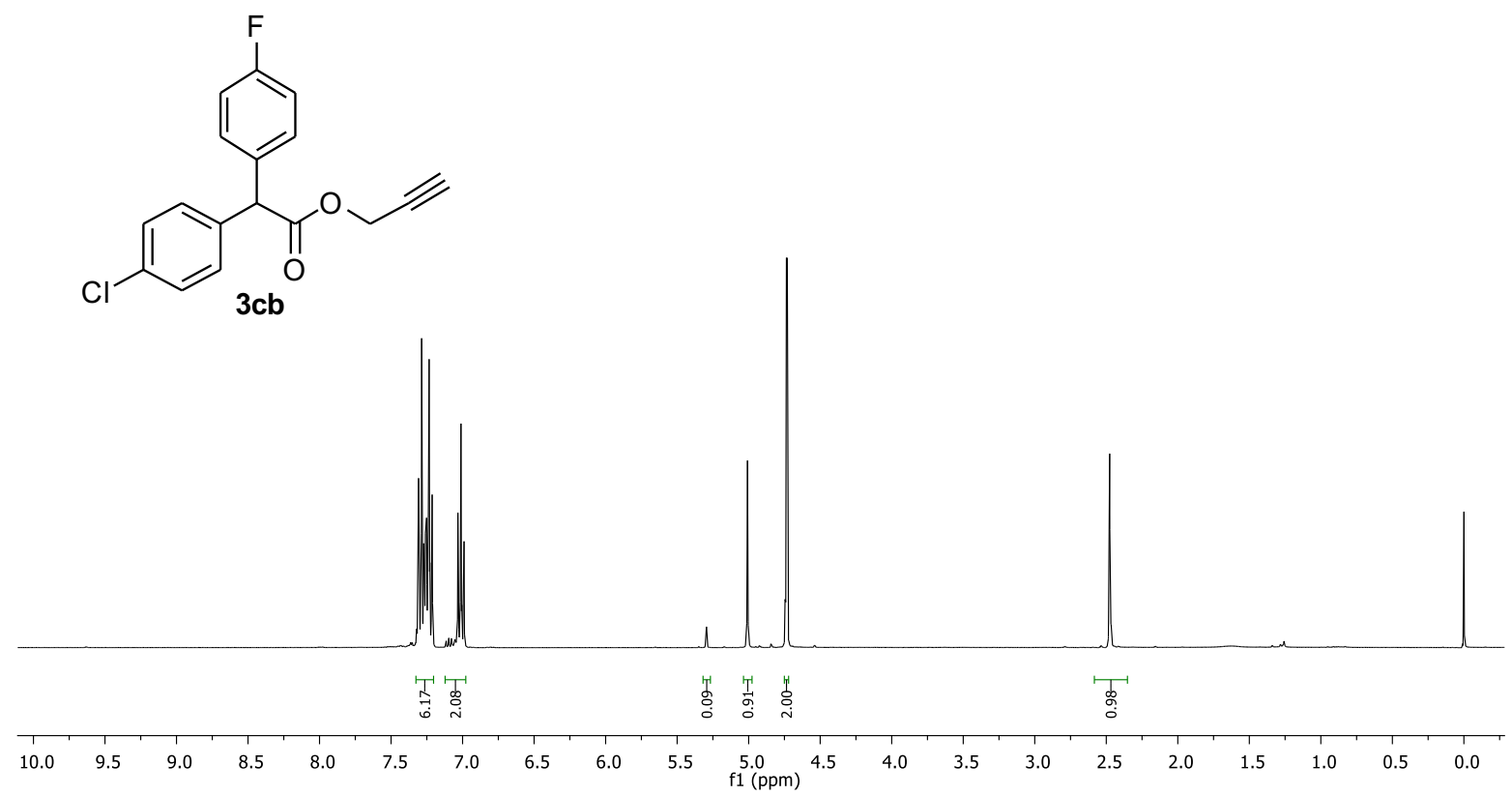


|
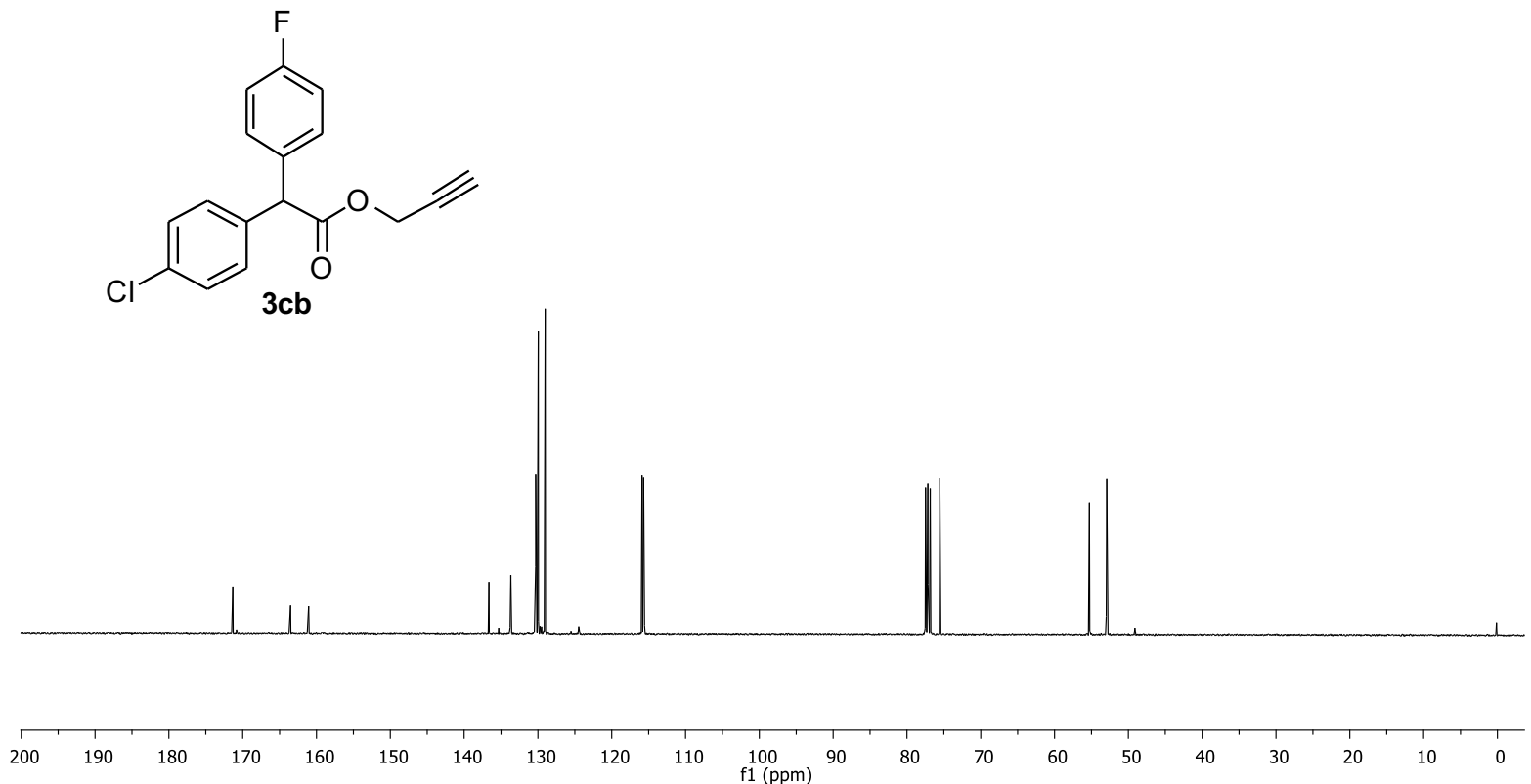

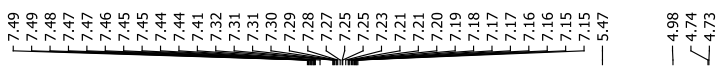
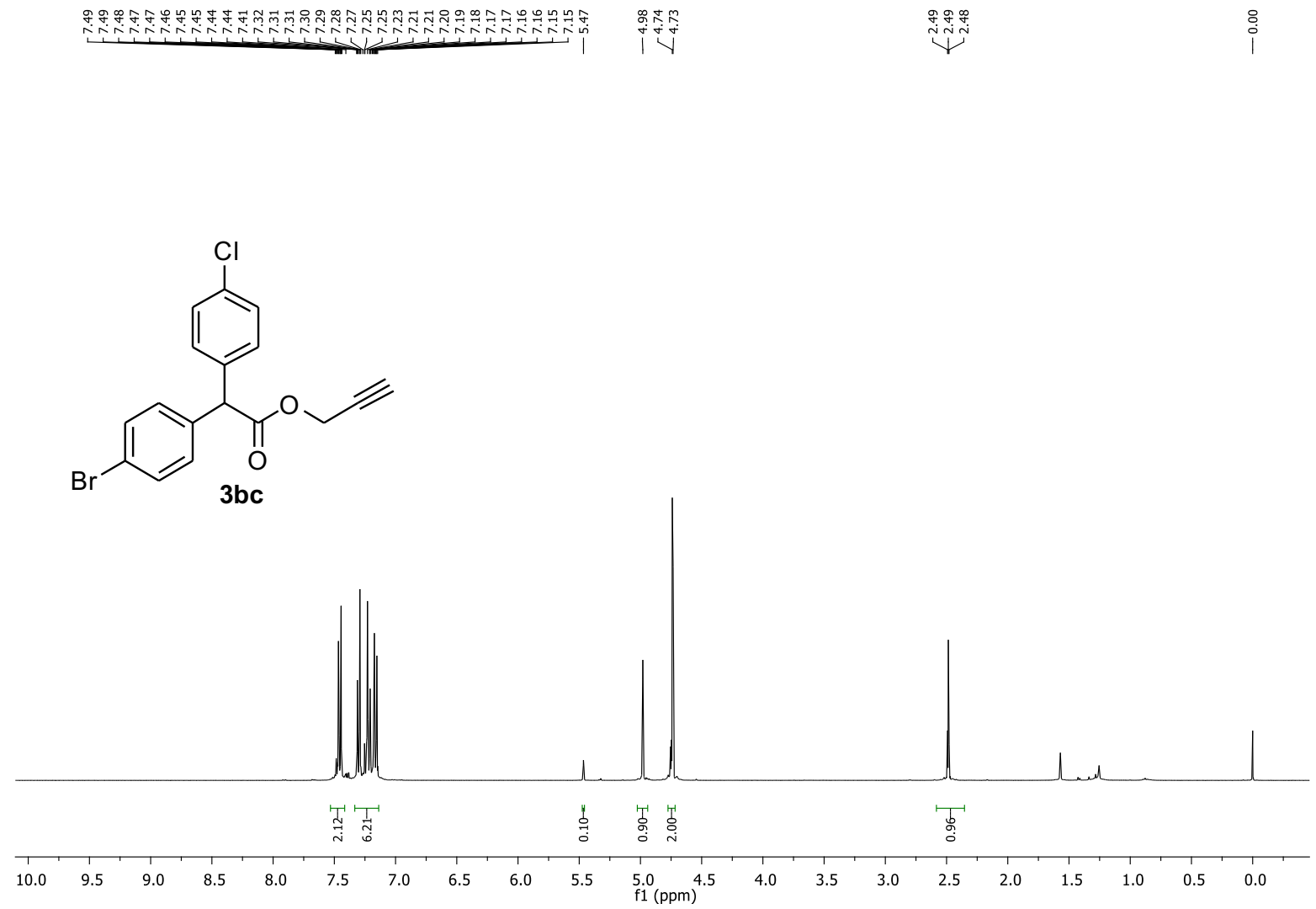


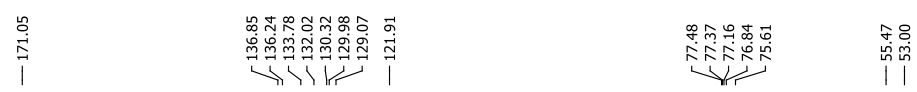
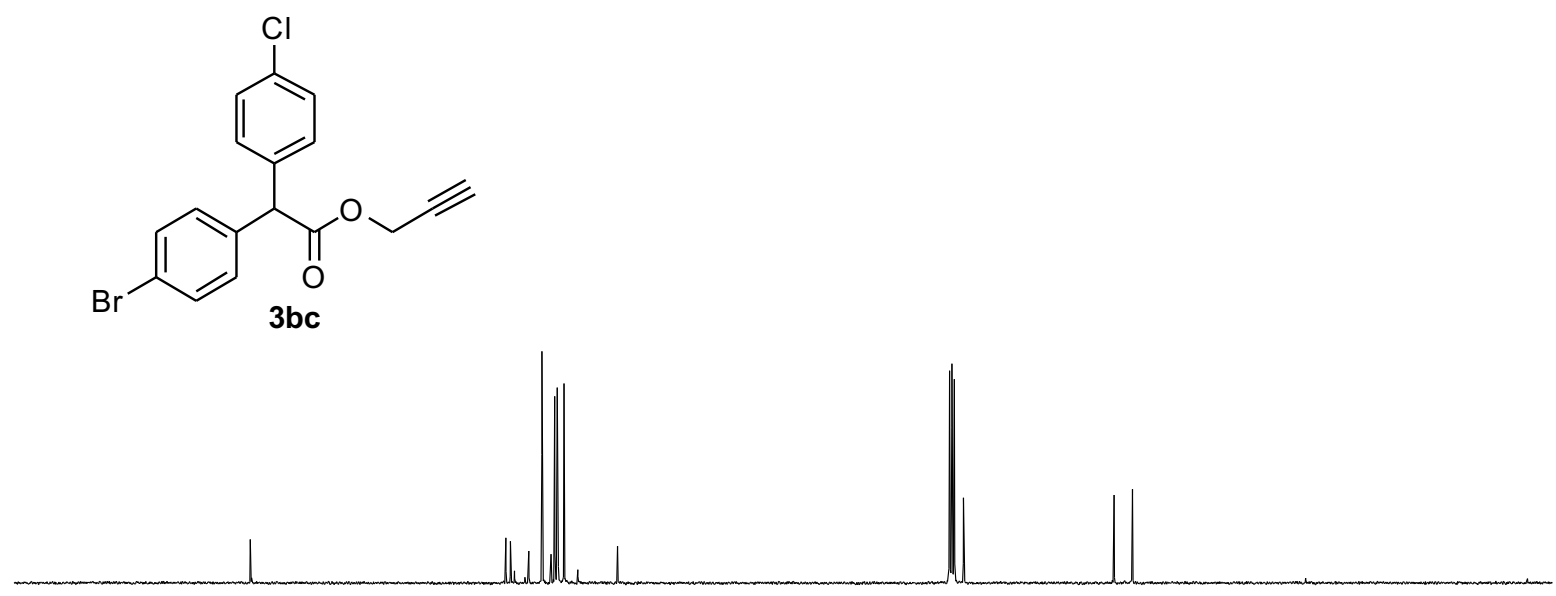

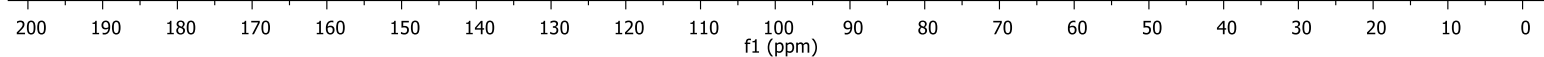

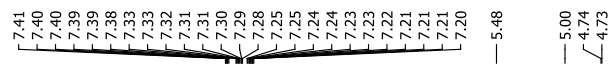
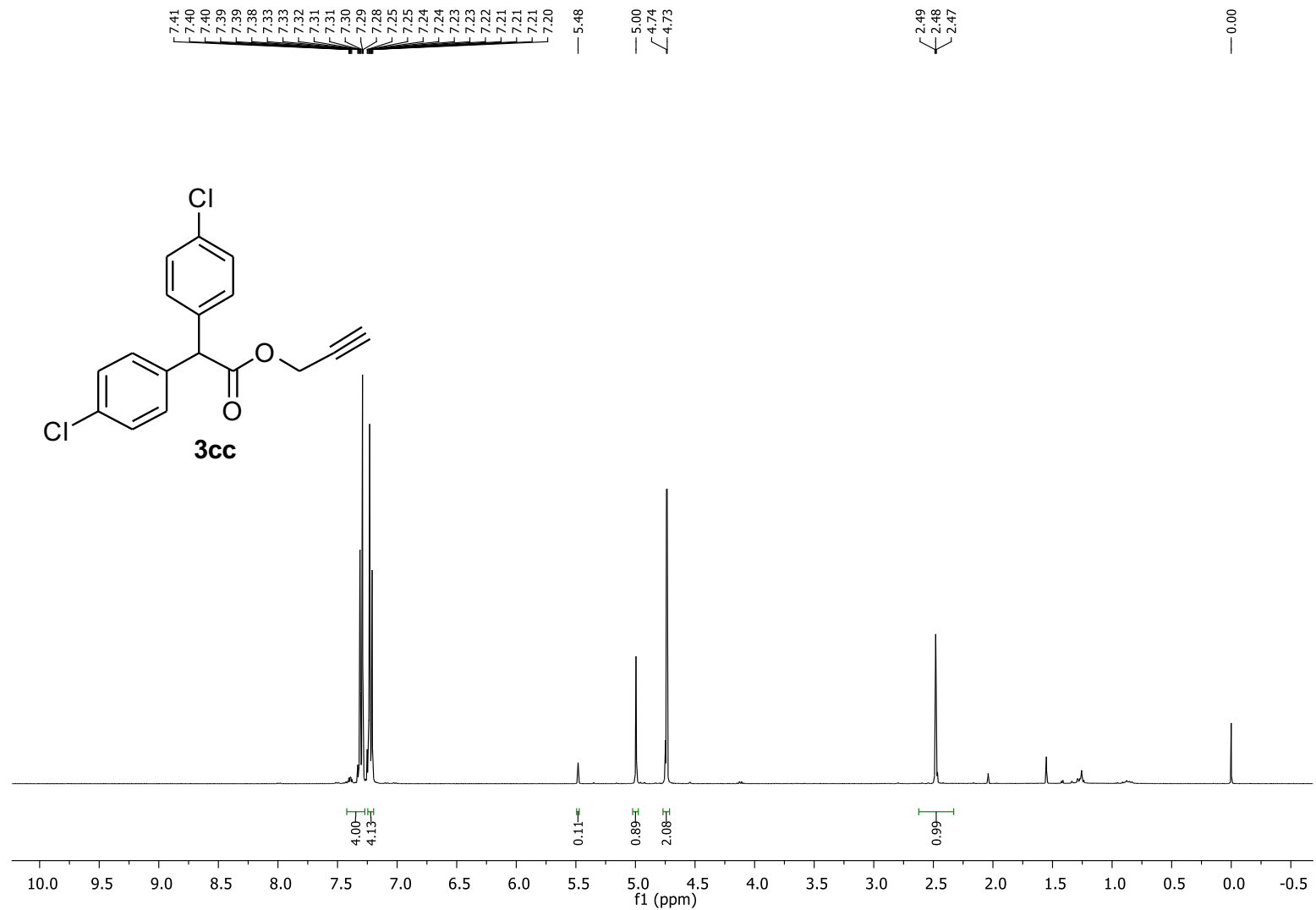


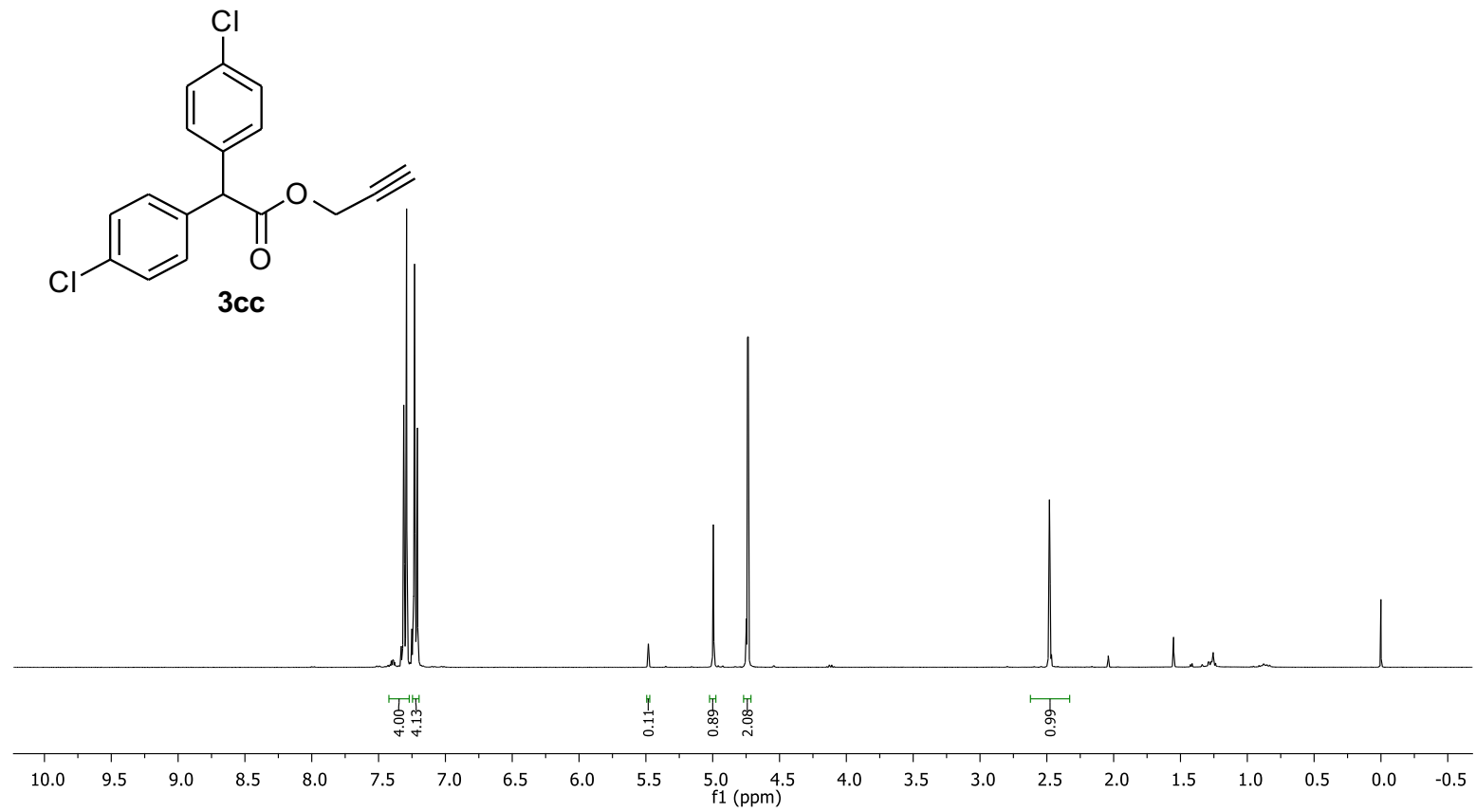

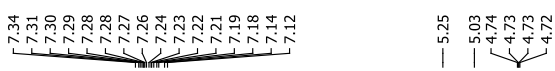

约约<smiles>C#CCOC(=O)C(c1ccccc1)c1ccc(C)cc1</smiles>

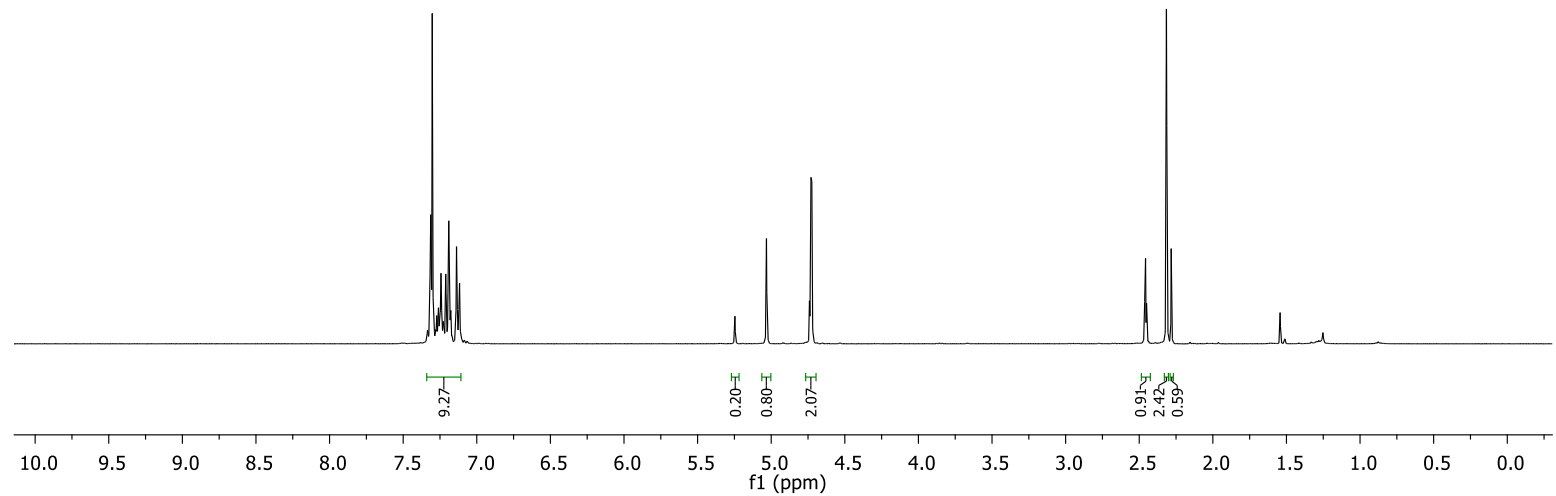



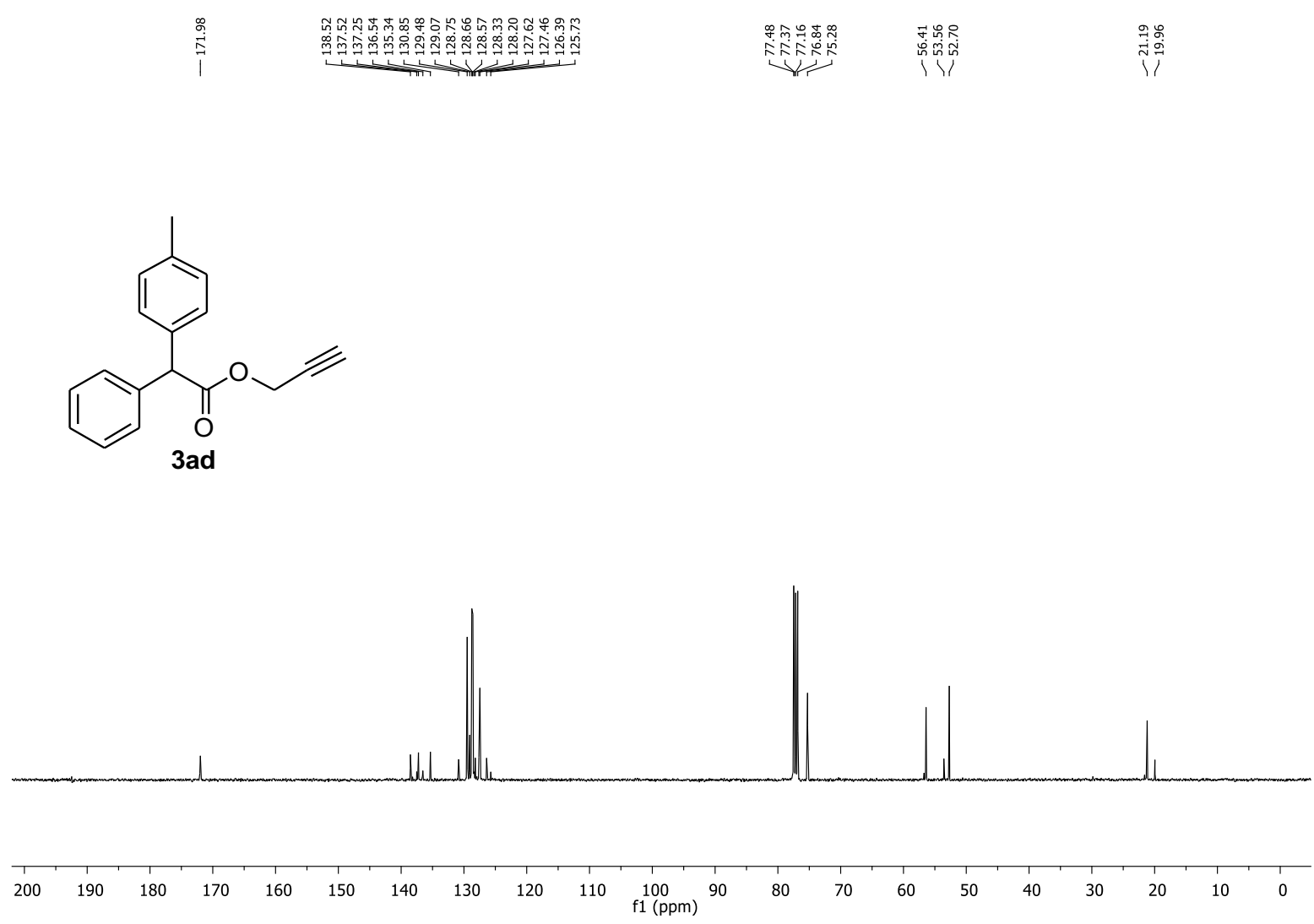

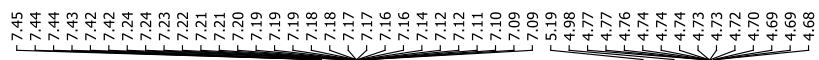

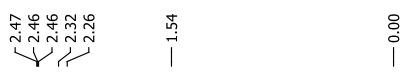

$\overbrace{3 b d}^{c}$

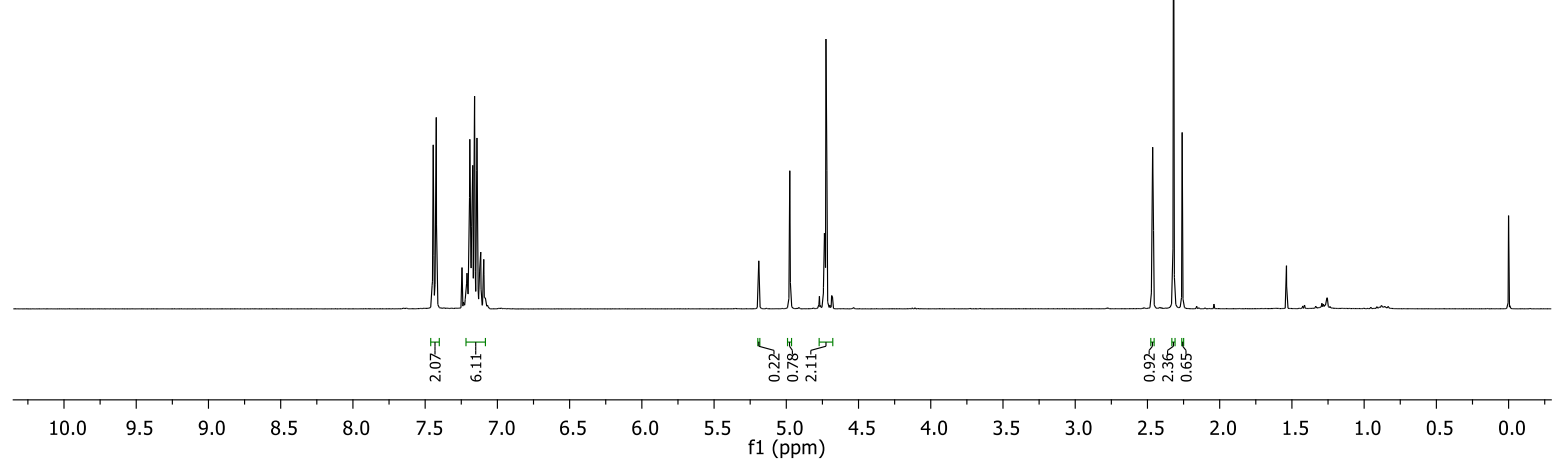


<smiles>C#CCOC(=O)C(c1ccc(C)cc1)c1ccc(Br)cc1</smiles>

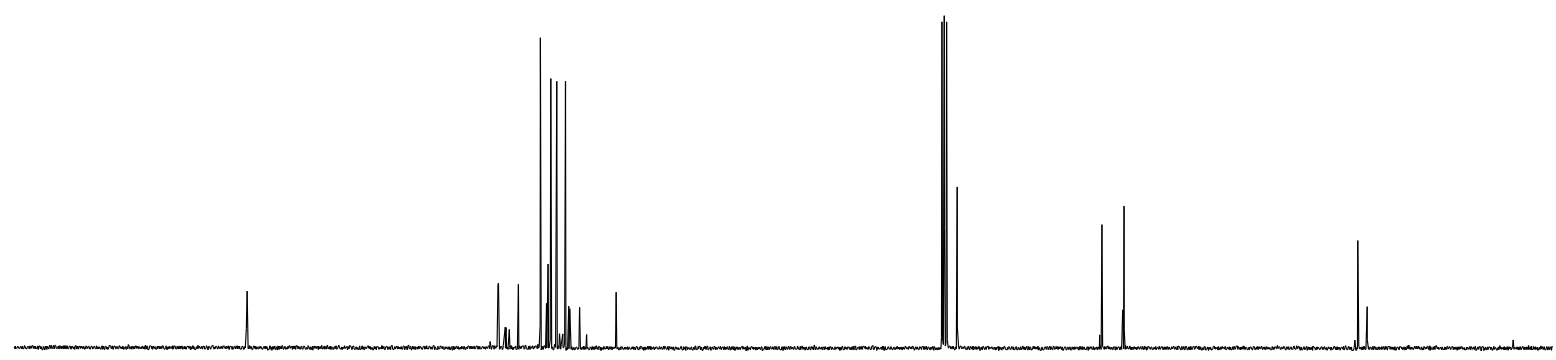

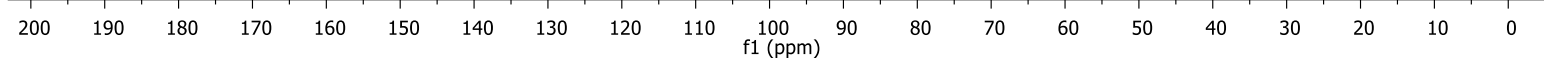<smiles>C#CCOC(=O)C(c1ccccc1)c1ccc(C)c(C)c1</smiles>

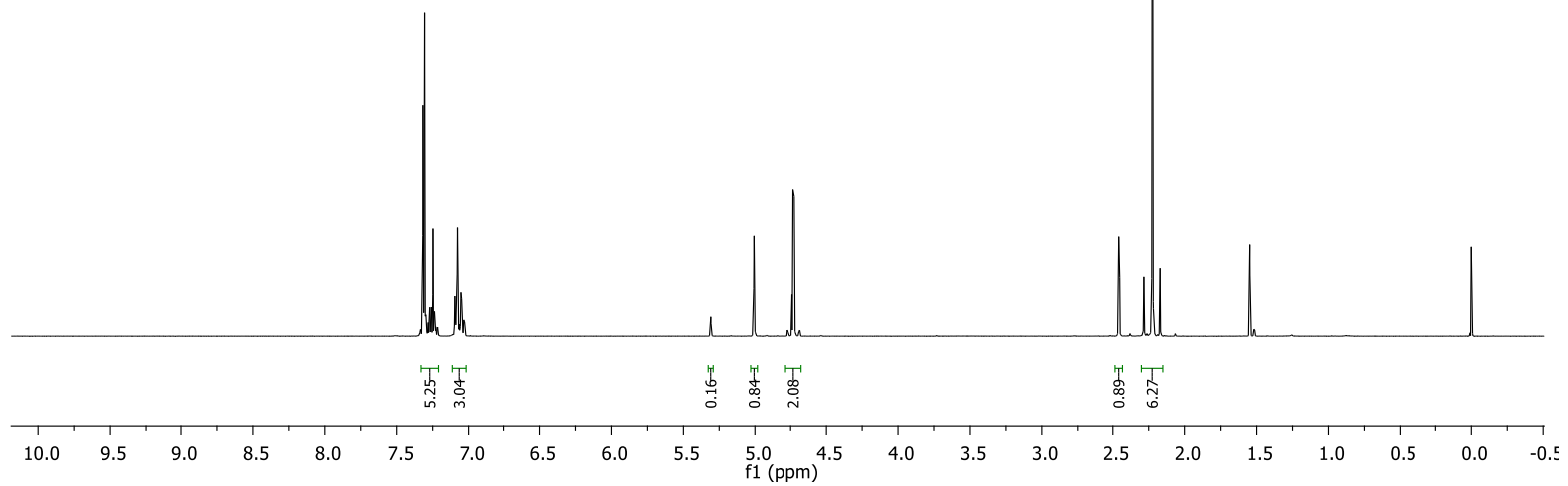




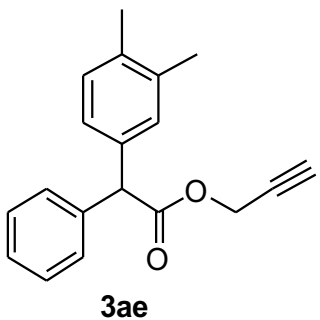<smiles>C#CCOC(=O)C(c1ccc(Br)cc1)c1ccc(C)c(C)c1</smiles>

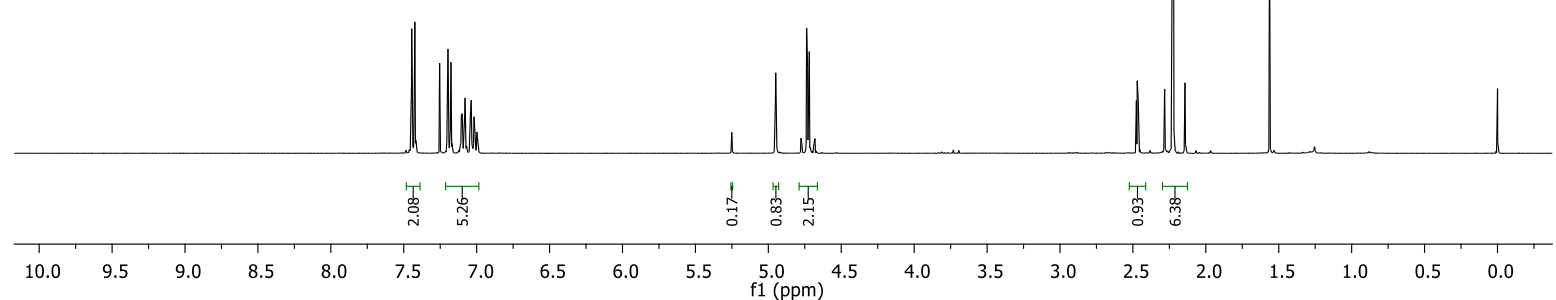




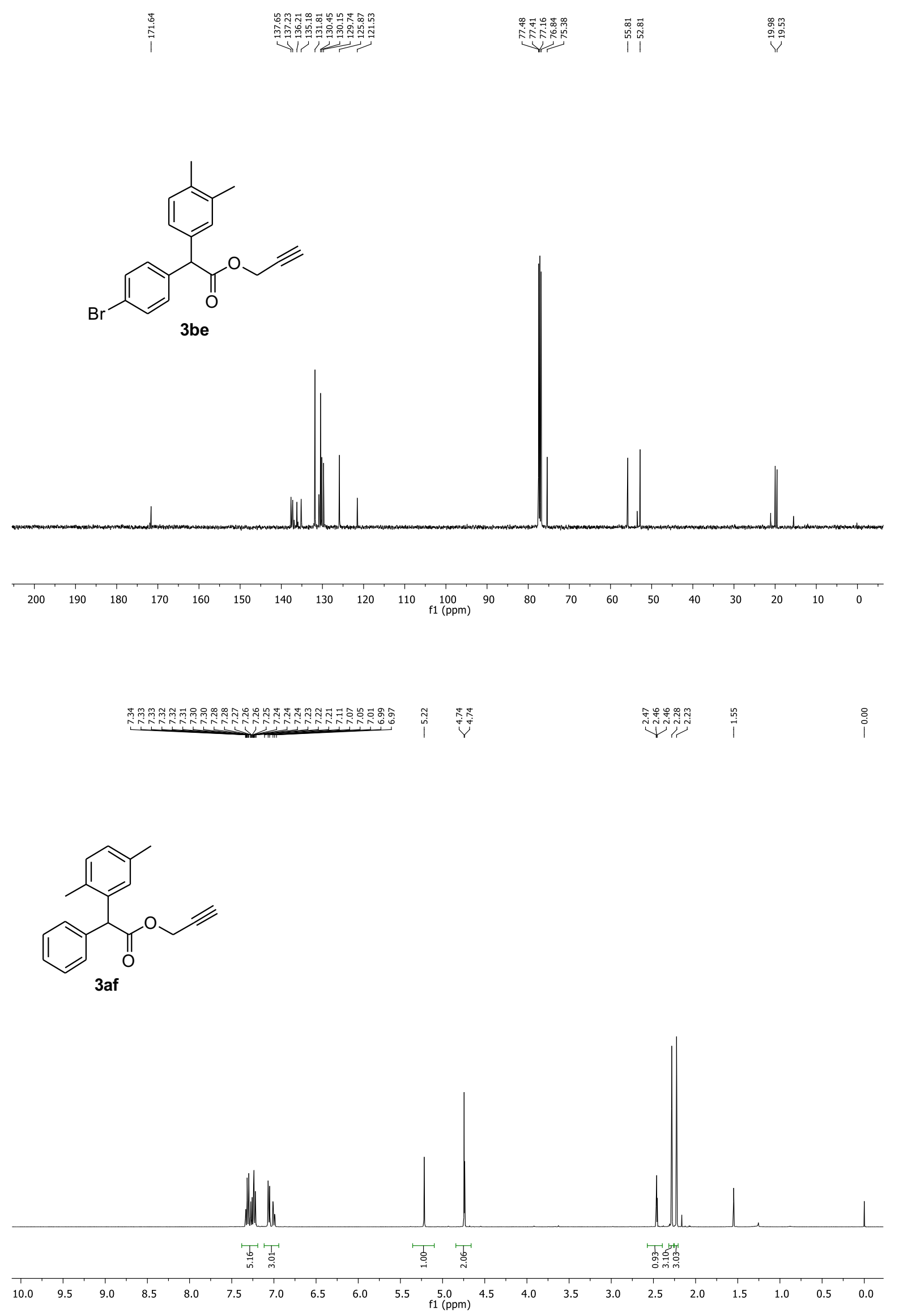




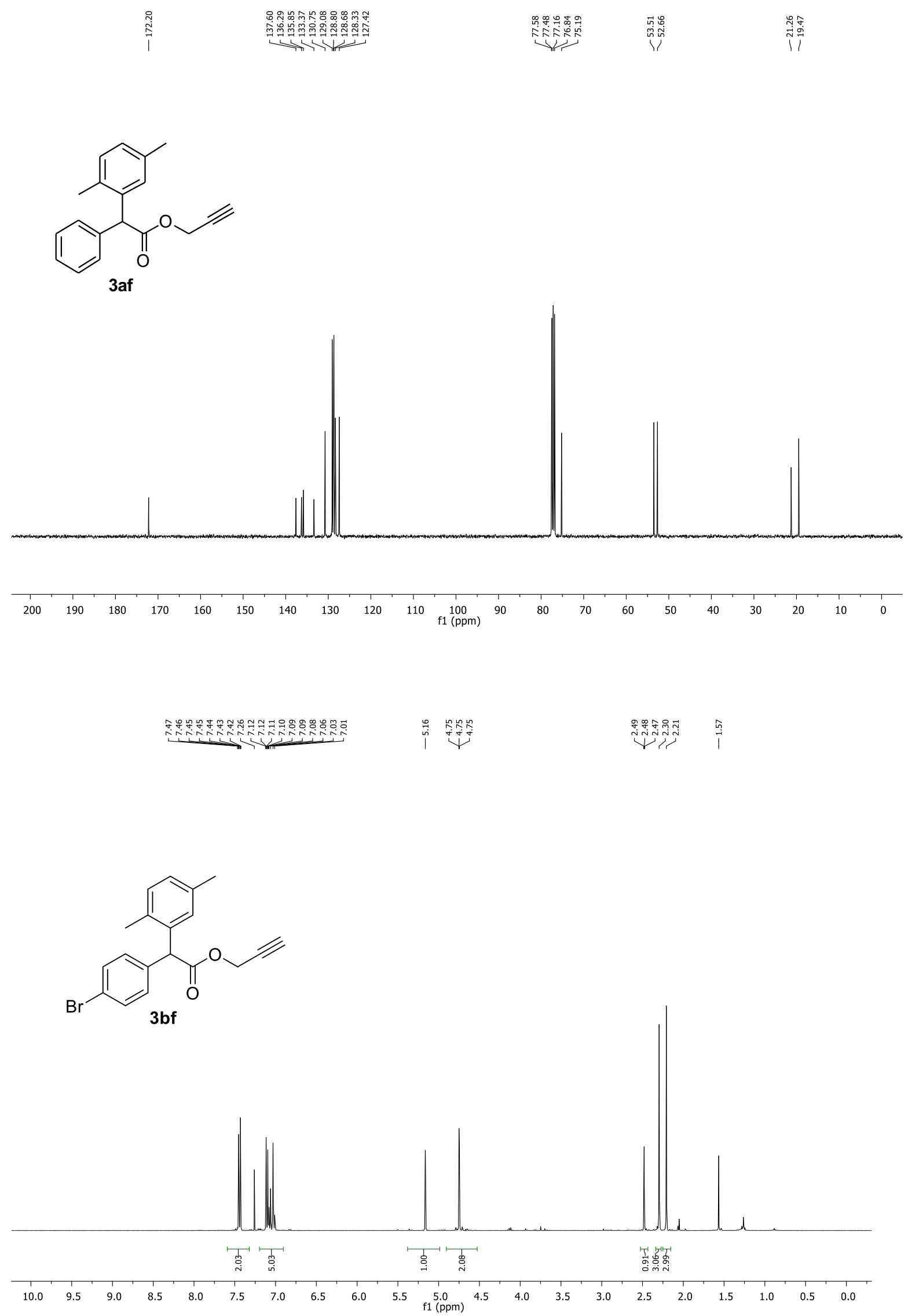



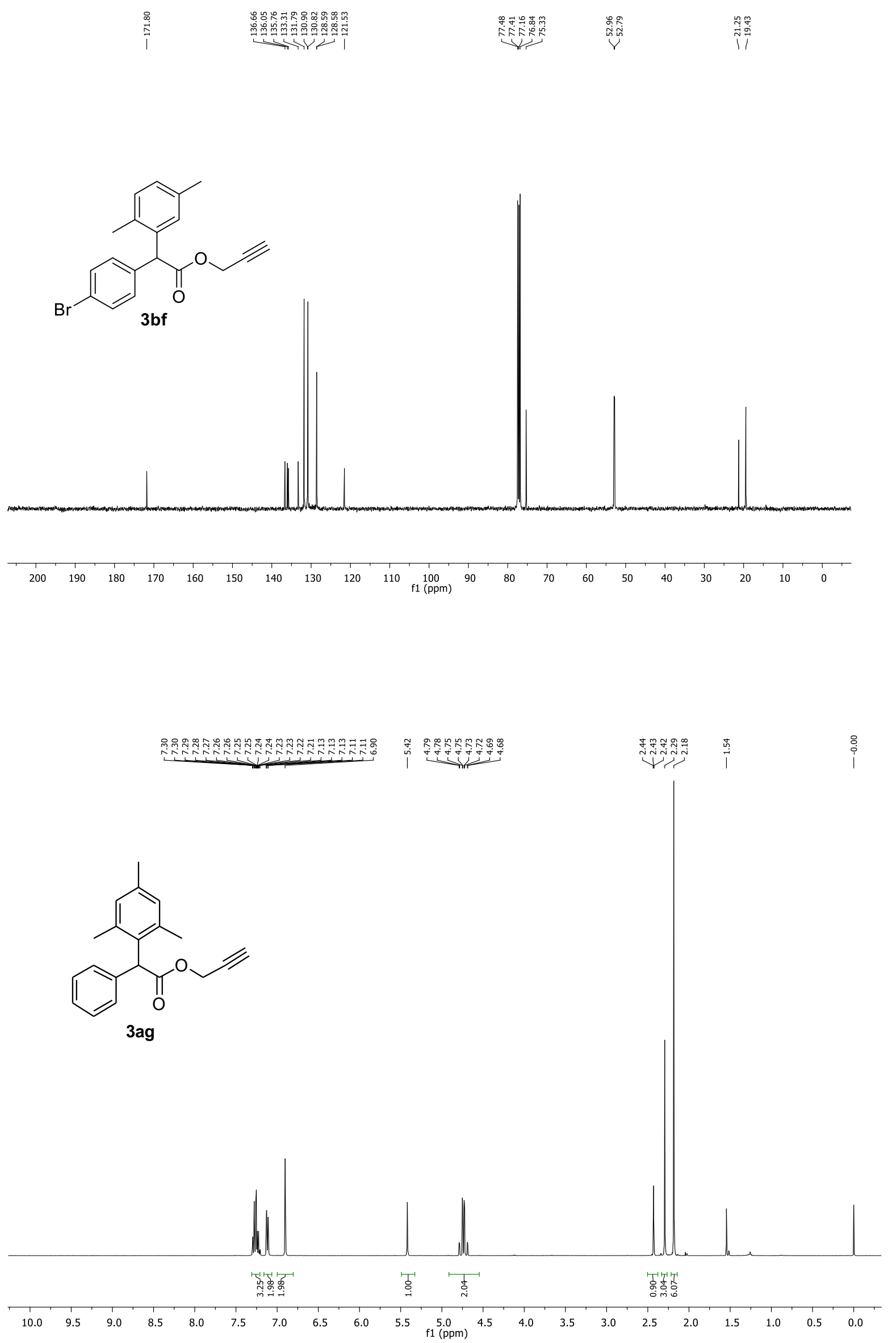


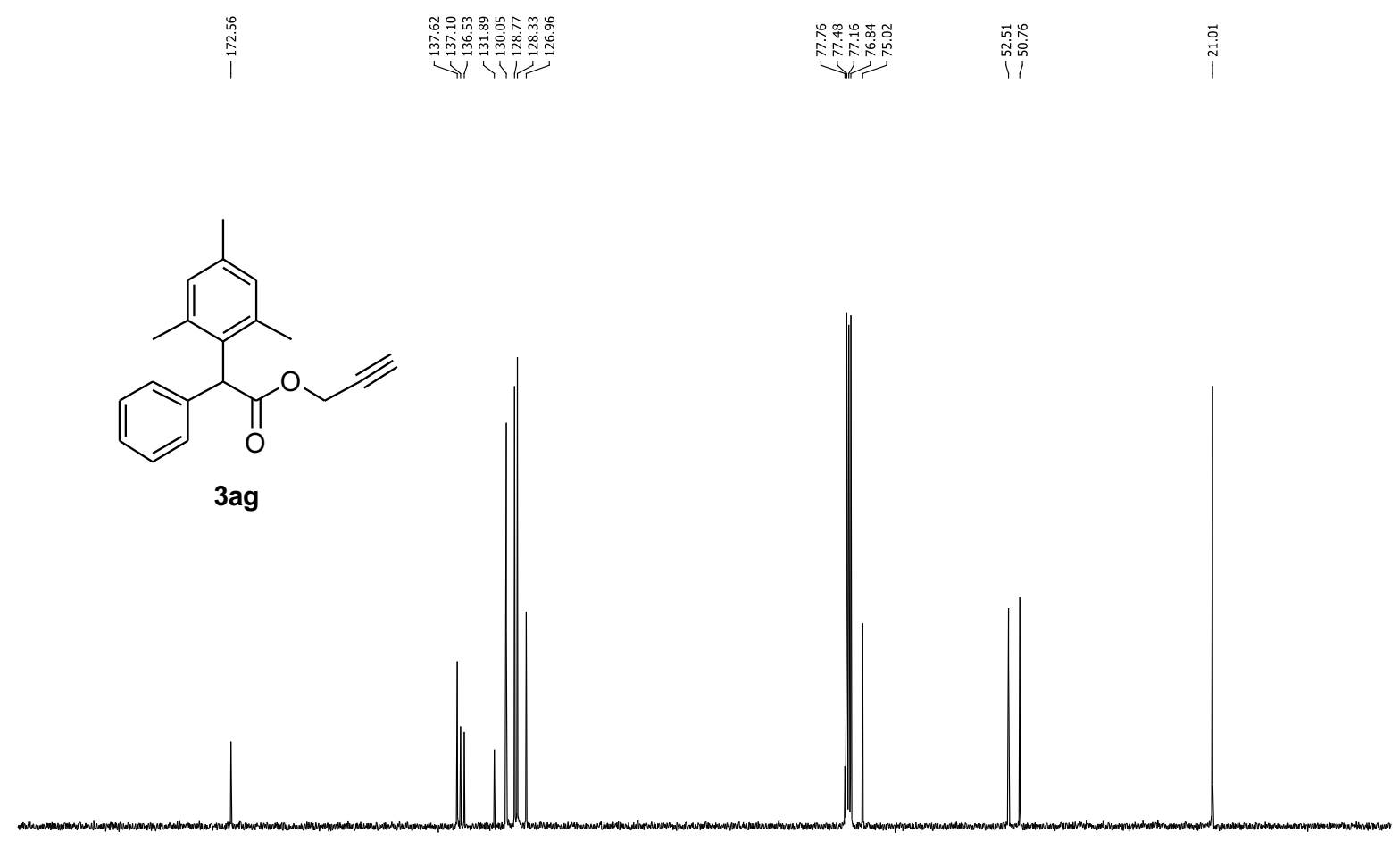

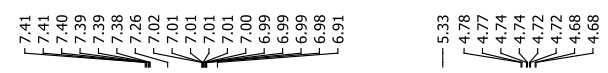

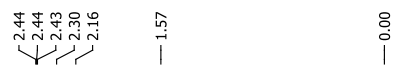<smiles>C#CCOC(=O)C(c1ccc(Br)cc1)c1c(C)cc(C)cc1C</smiles>

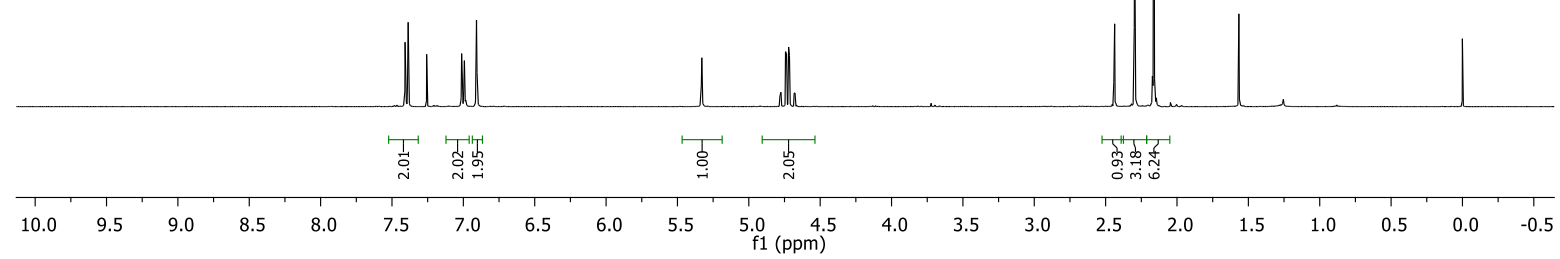




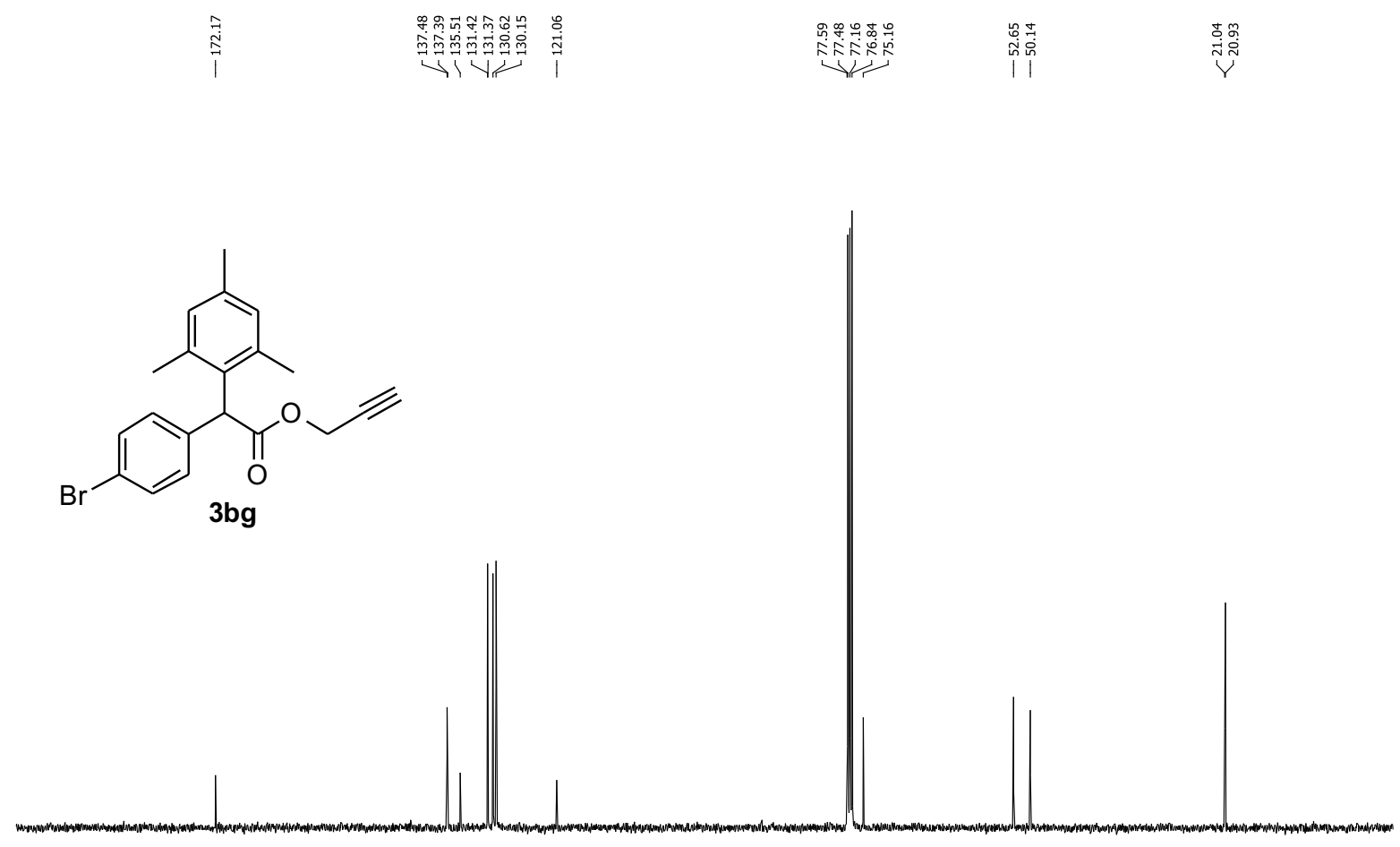

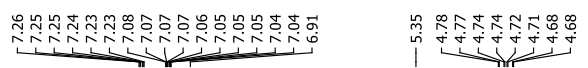<smiles>C#CCOC(=O)C(c1ccc(Cl)cc1)c1c(C)cc(C)cc1C</smiles>

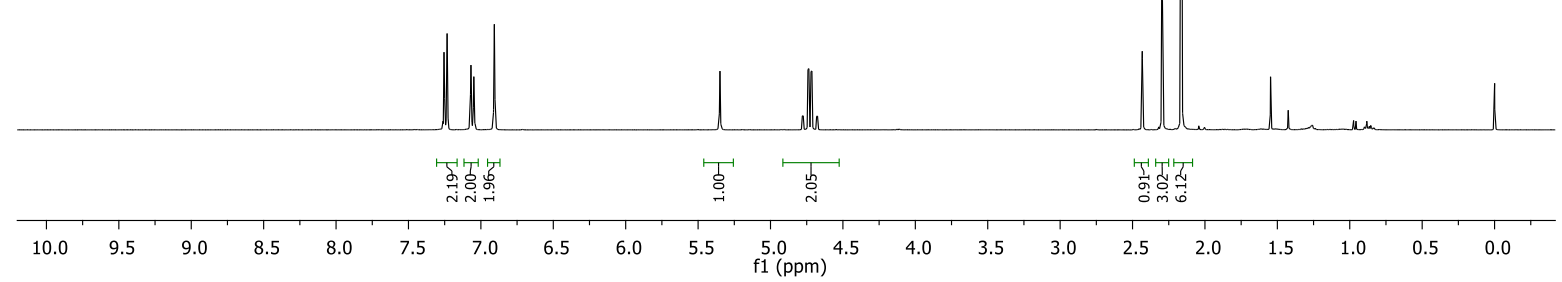




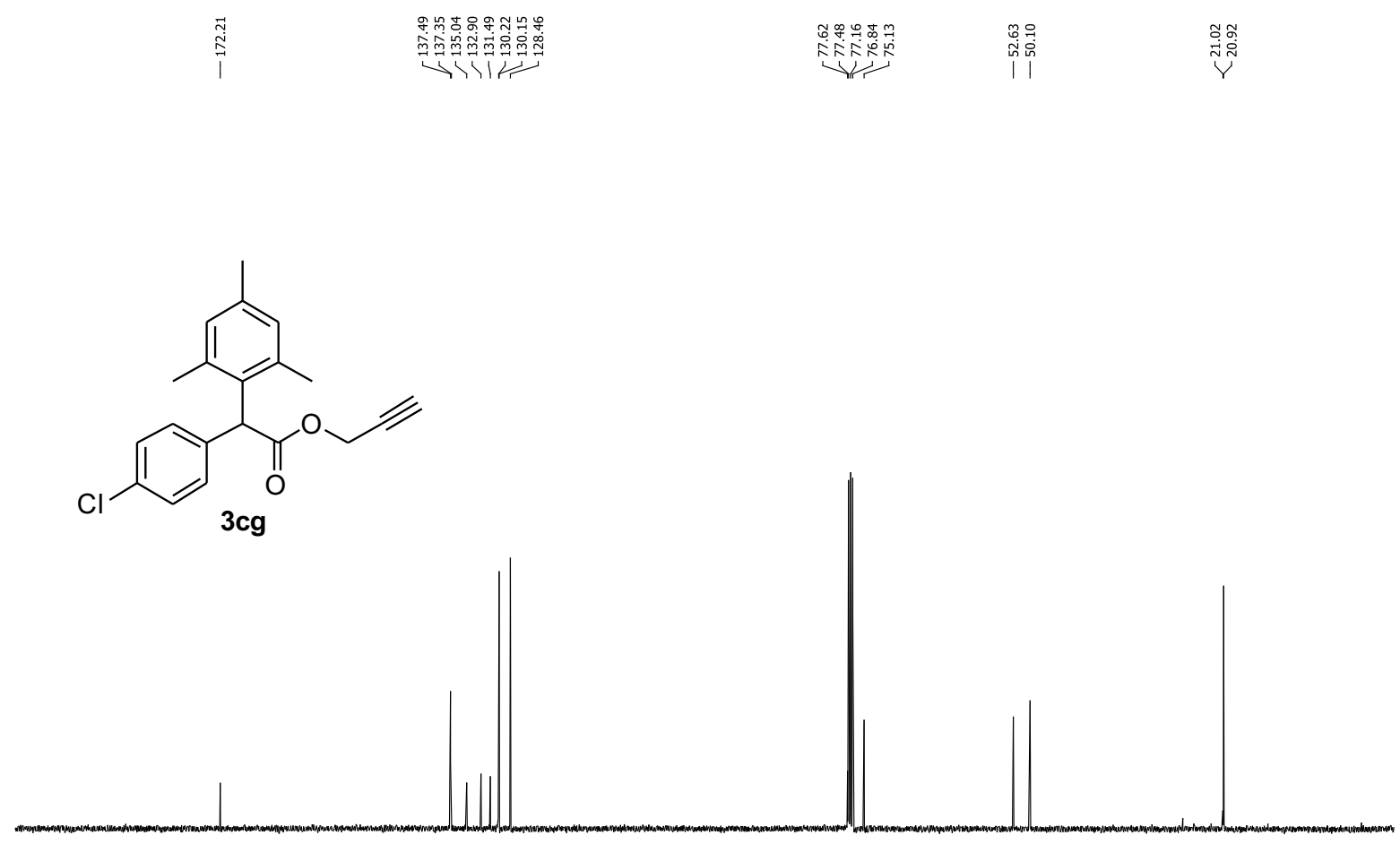

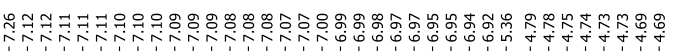
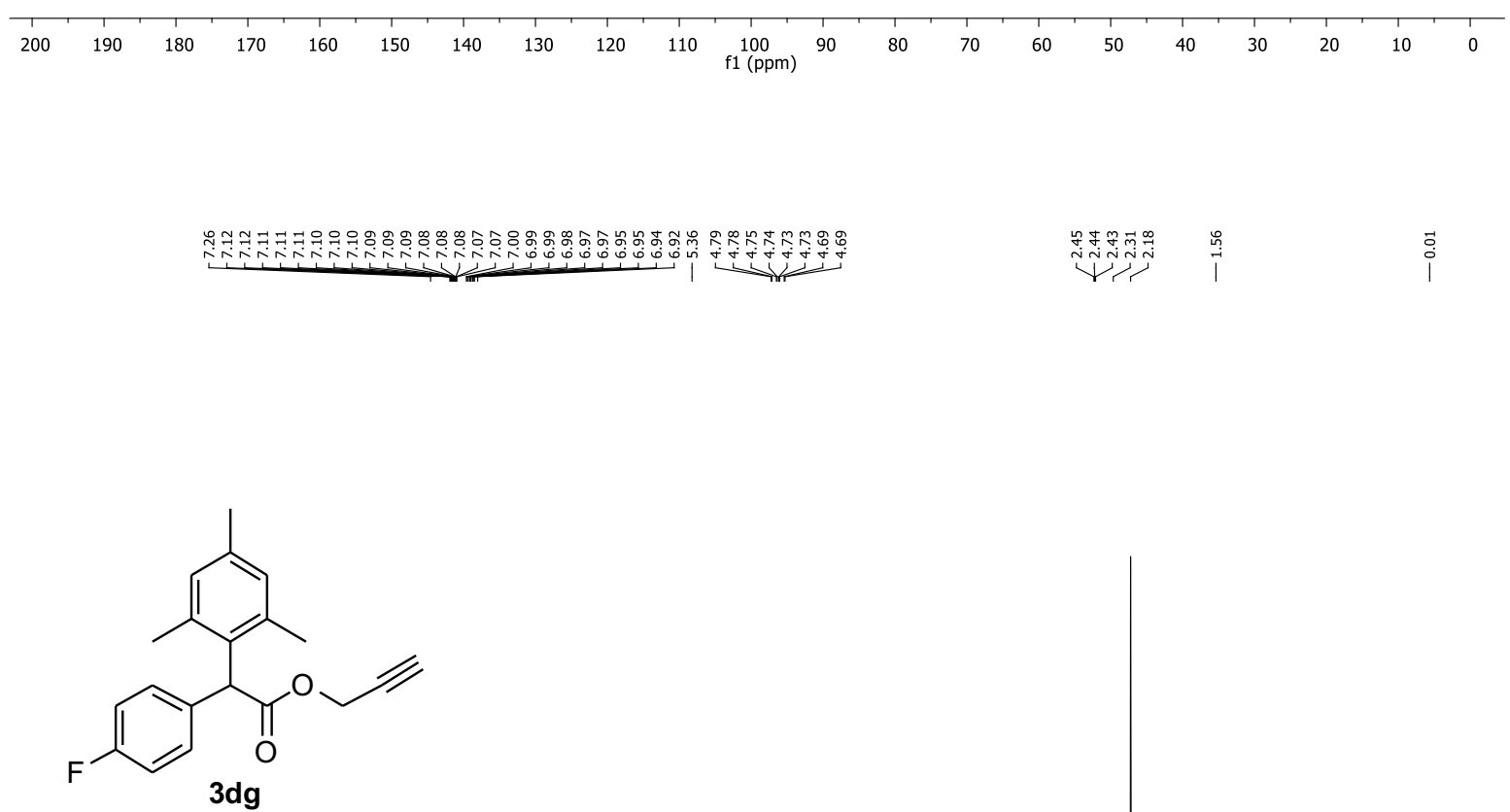

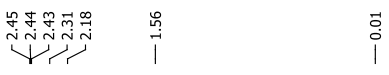

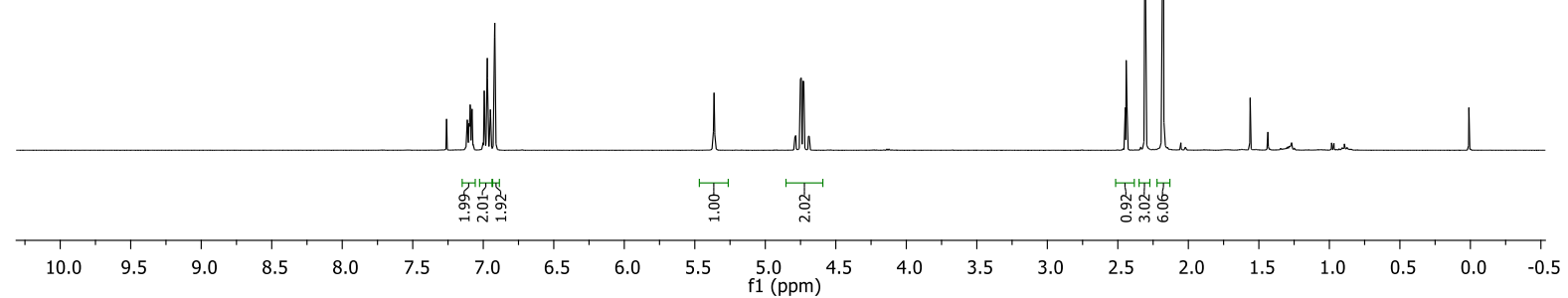



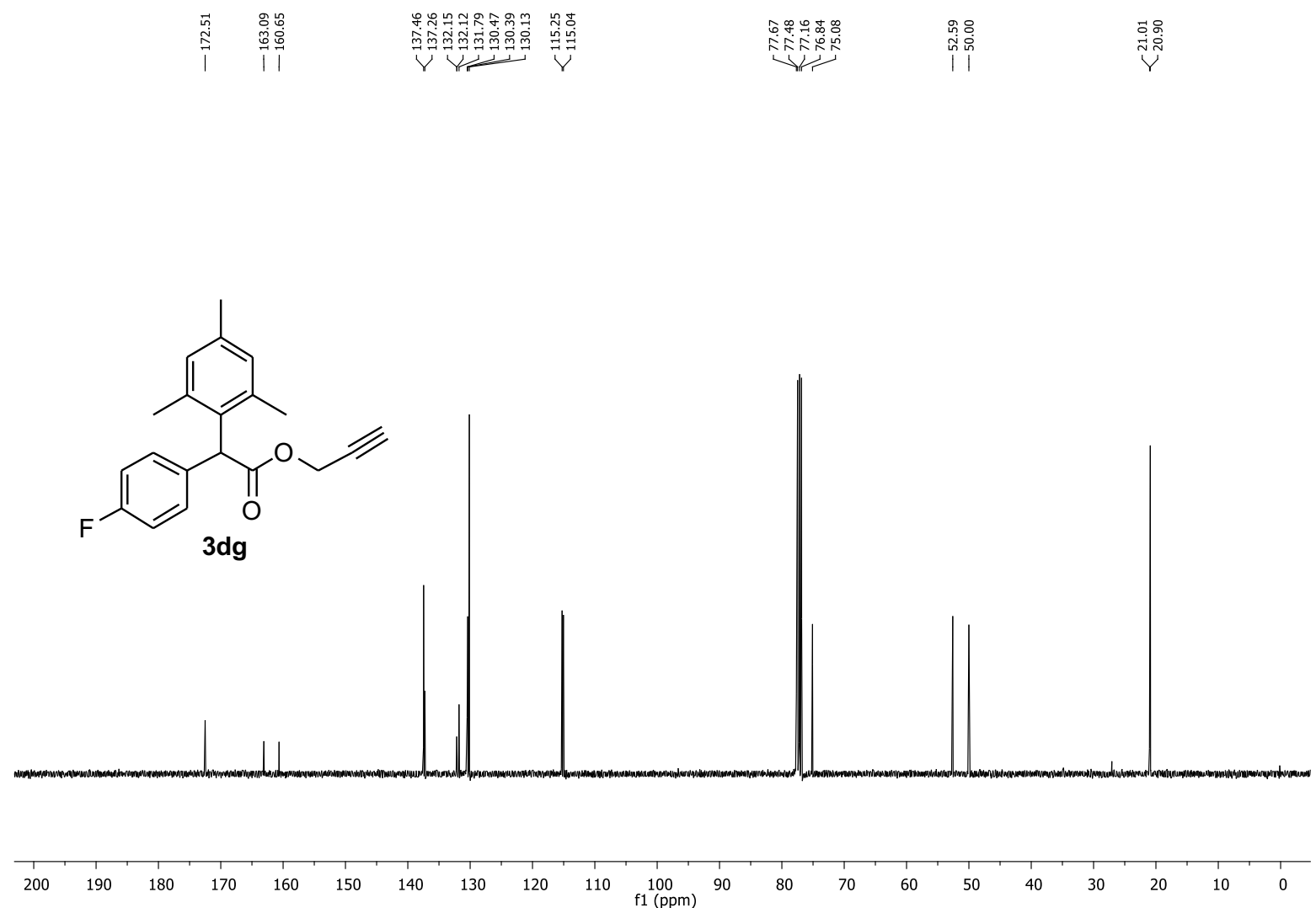

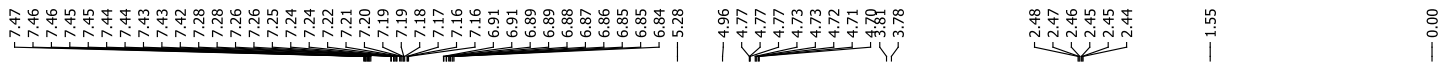

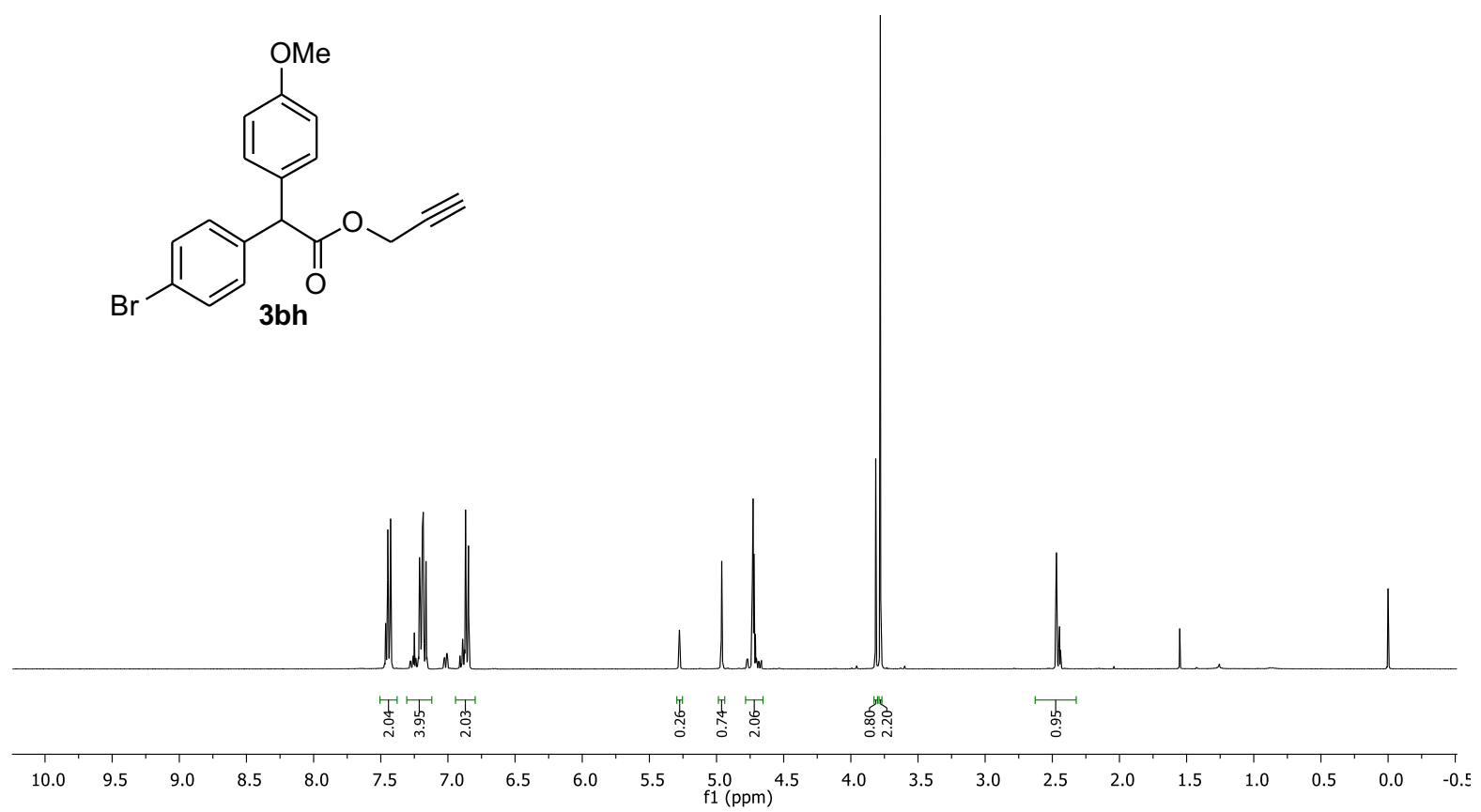



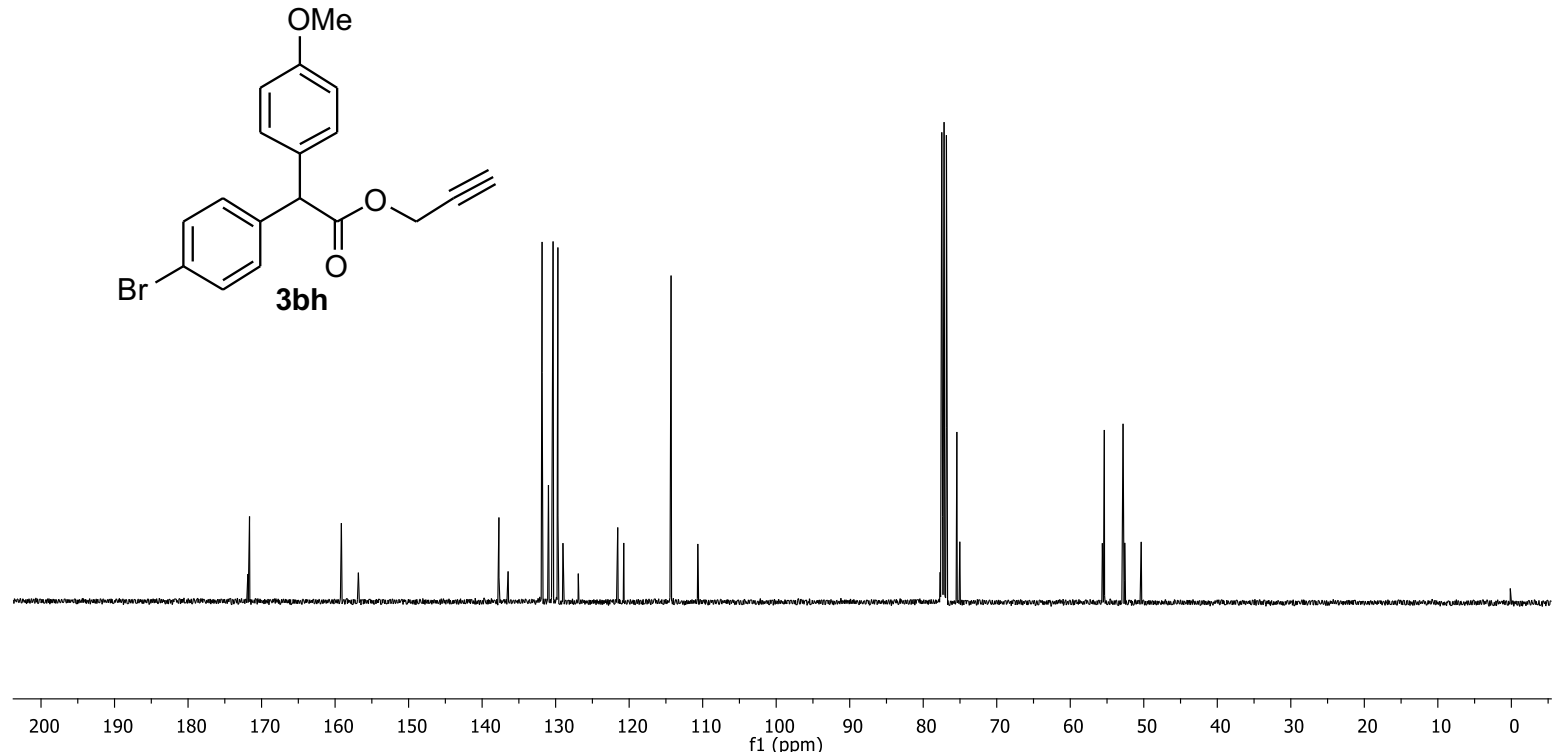<smiles>C#CCOC(=O)C(c1ccccc1)c1cc(OC)ccc1OC</smiles>

3ai

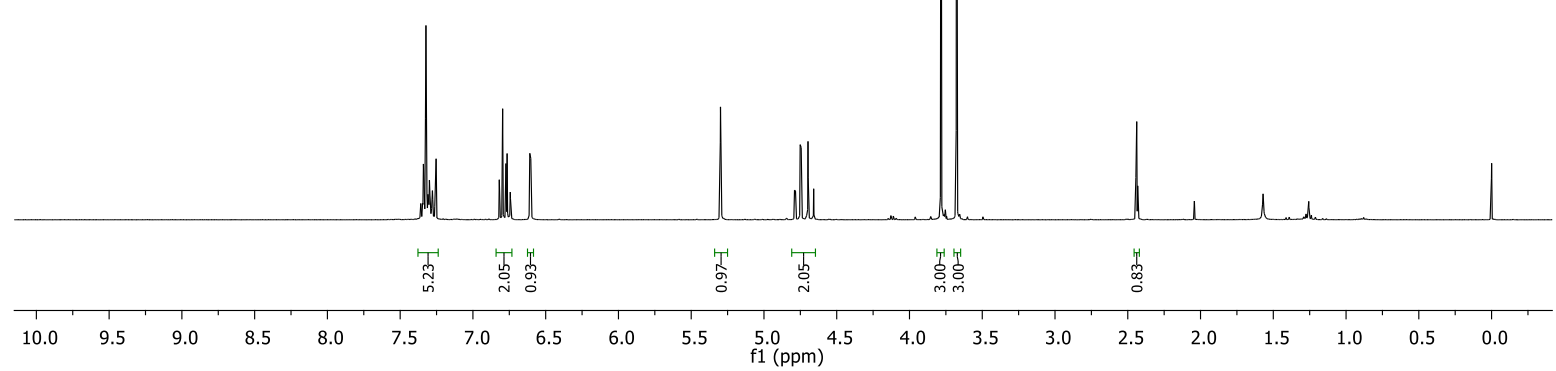




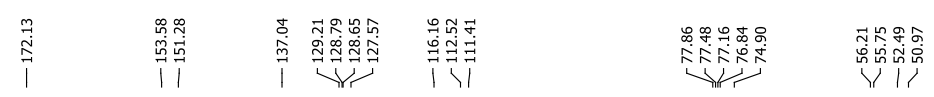

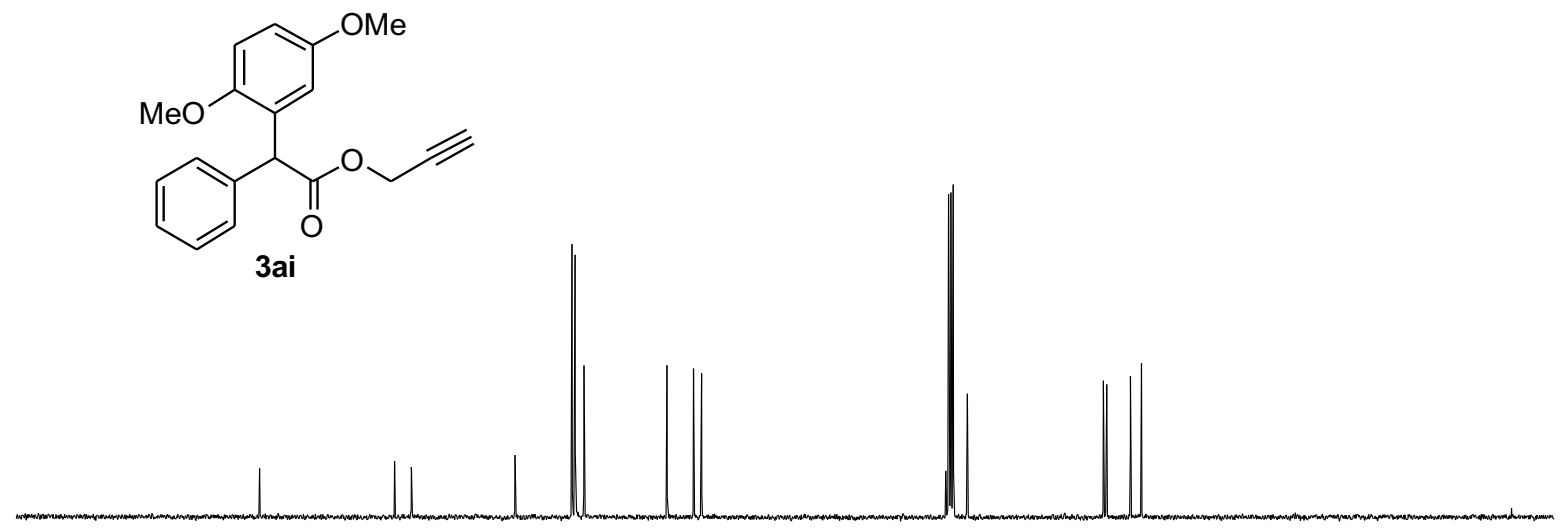

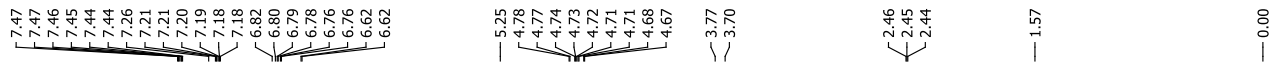<smiles>C#CCOC(=O)C(c1ccc(Br)cc1)c1cc(OC)ccc1OC</smiles>

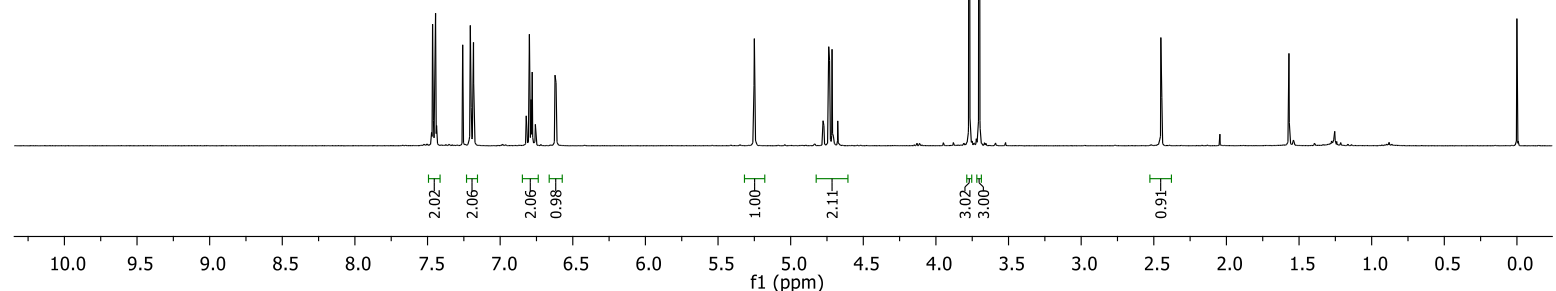




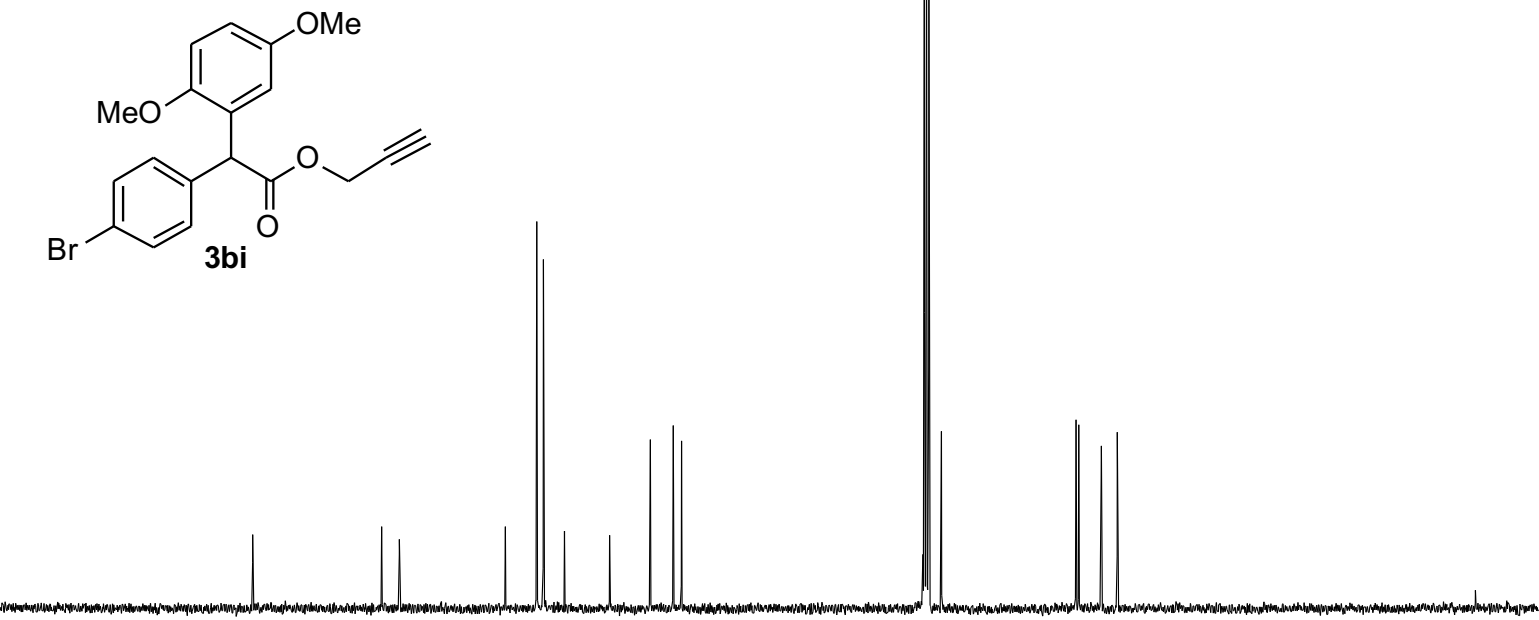

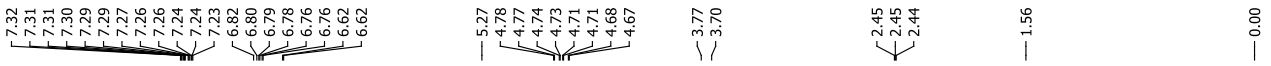<smiles>C#CCOC(=O)C(c1ccc(Cl)cc1)c1cc(OC)ccc1OC</smiles>

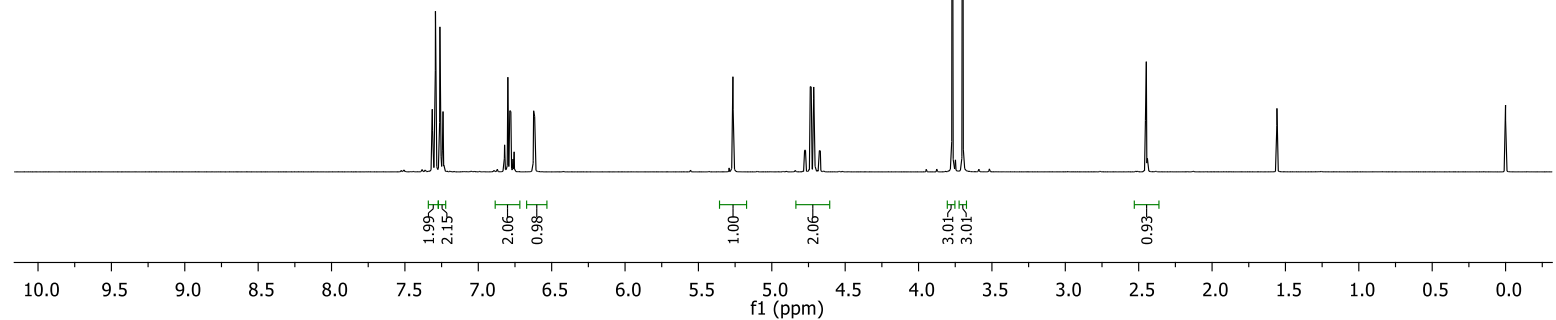


|<smiles>C#CCOC(=O)C(c1ccc(Cl)cc1)c1cc(OC)ccc1OC</smiles>

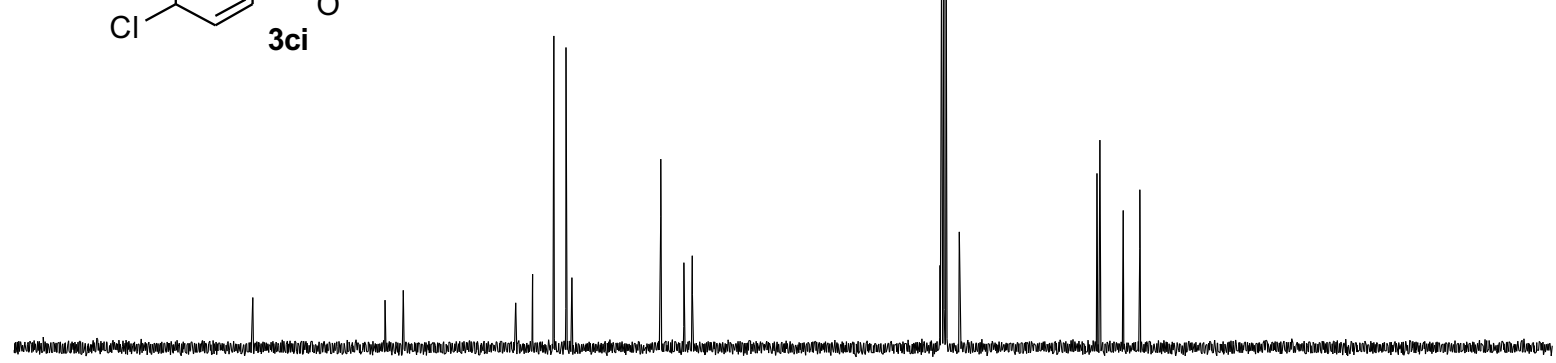

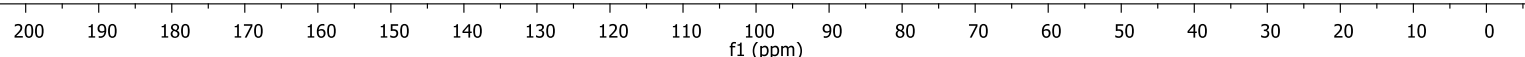

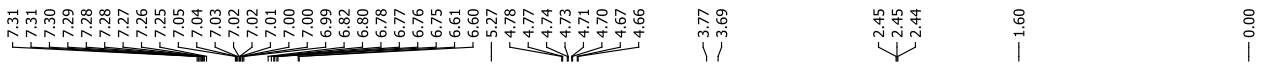<smiles>C#CCOC(=O)C(c1ccc(F)cc1)c1cc(OC)ccc1OC</smiles>

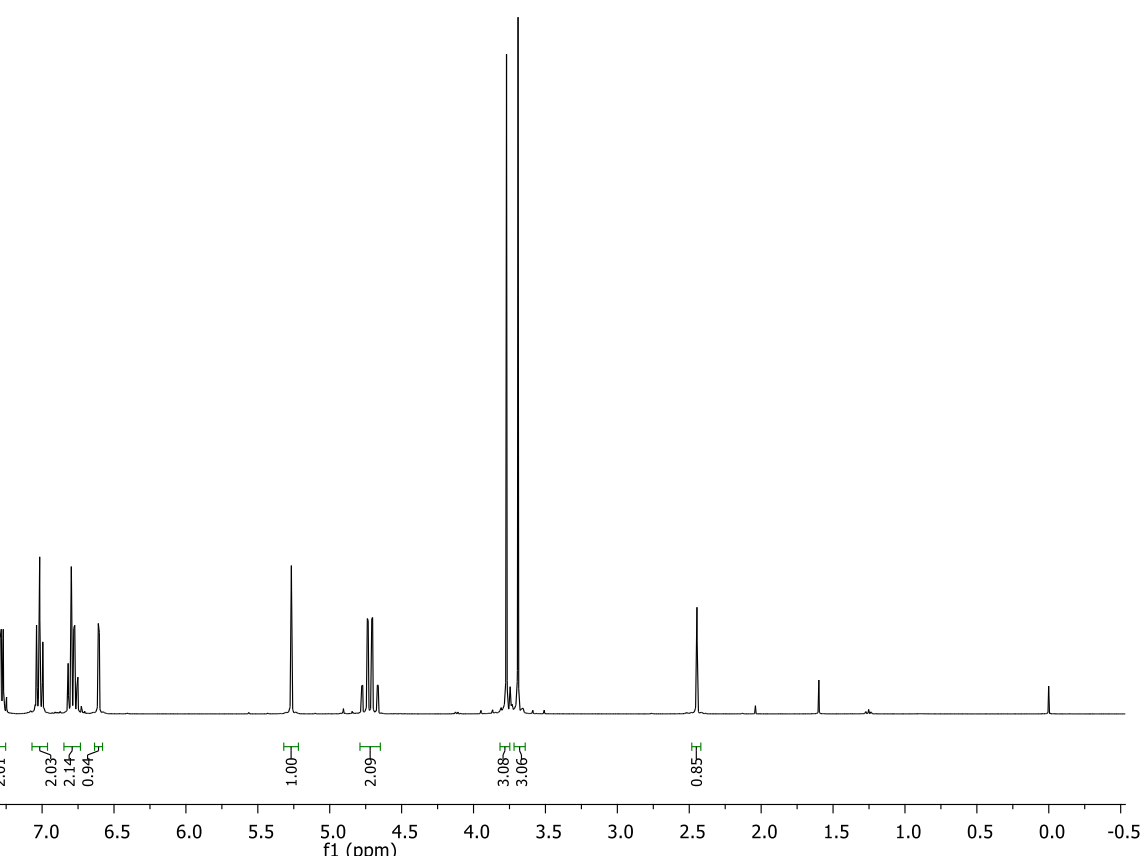




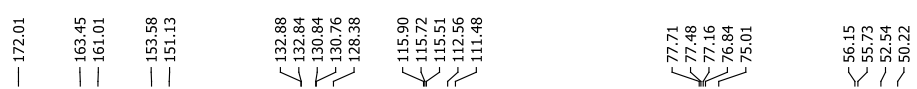<smiles>C#CCOC(=O)C(c1ccc(F)cc1)c1cc(OC)ccc1OC</smiles>

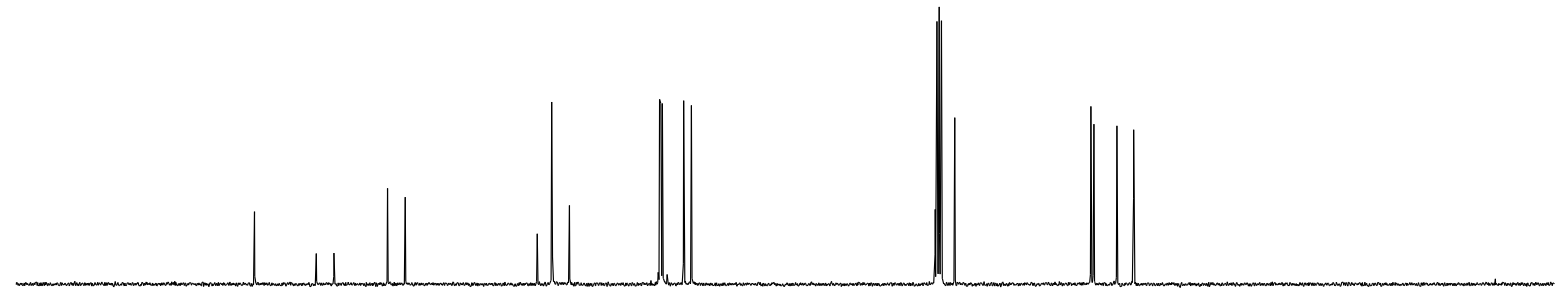

$\begin{array}{llllllllllllllllllllllll}200 & 190 & 180 & 170 & 160 & 150 & 140 & 130 & 120 & 110 & 100 & 90 & 80 & 70 & 60 & 50 & 40 & 30 & 20 & 10 & 0\end{array}$

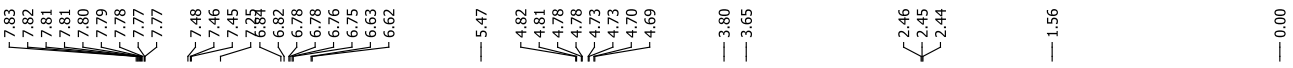<smiles>C#CCOC(=O)C(c1cc(OC)ccc1OC)c1cccc2ccccc12</smiles>

$3 g i$

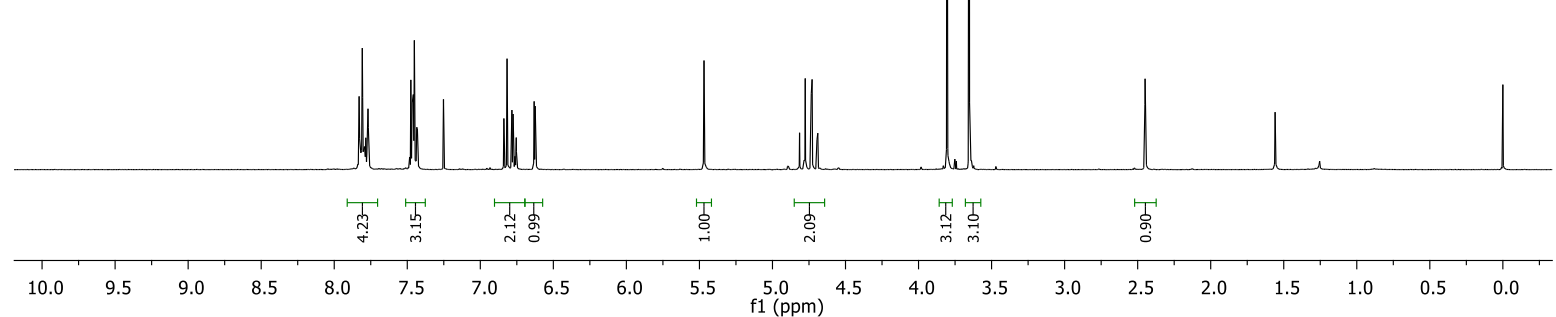



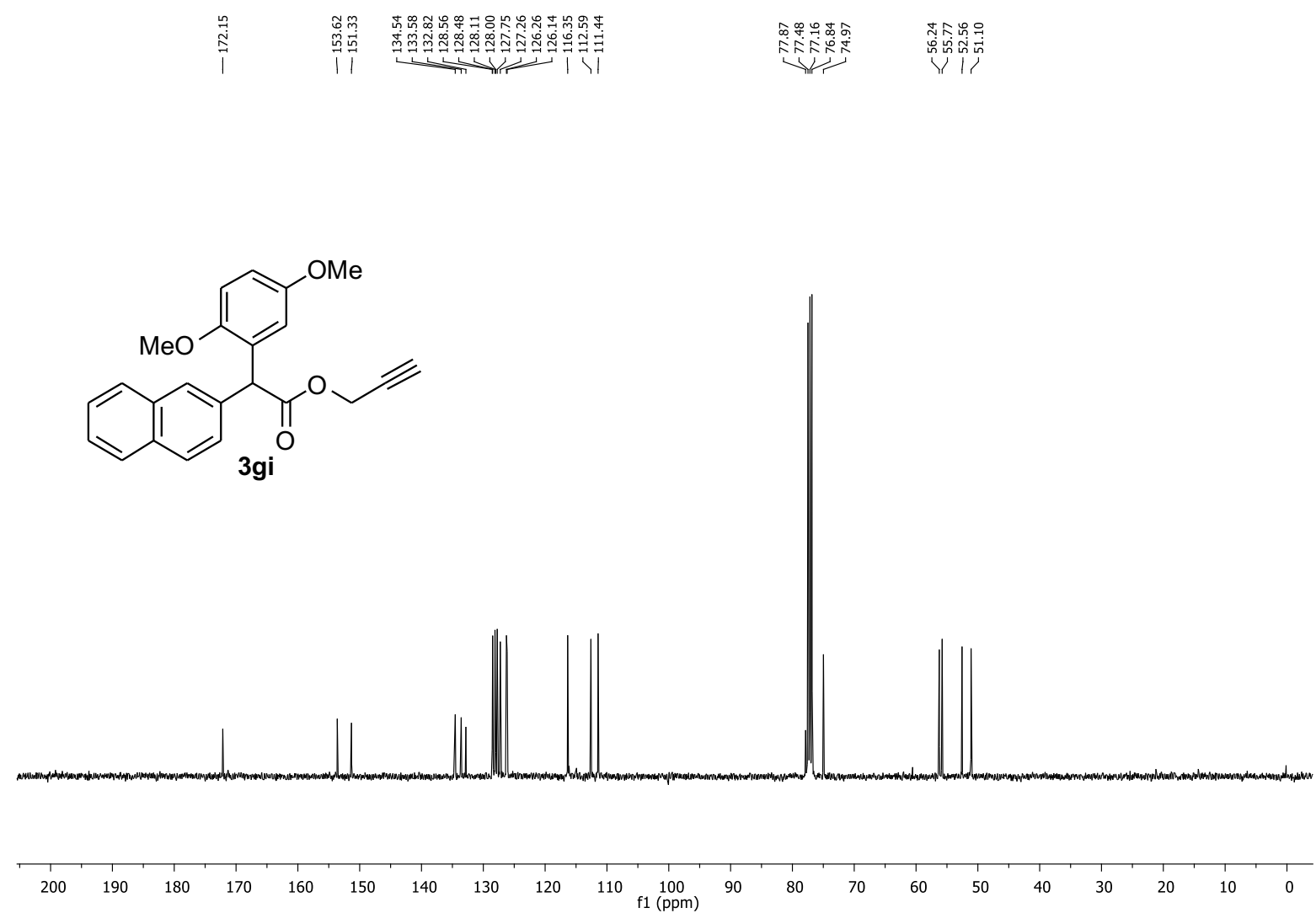

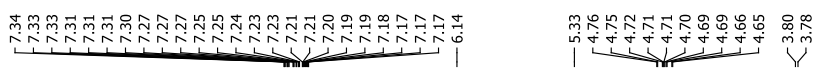

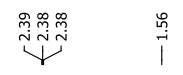<smiles>C#CCOC(=O)C(c1ccccc1)c1c(OC)cc(OC)cc1OC</smiles>

3aj

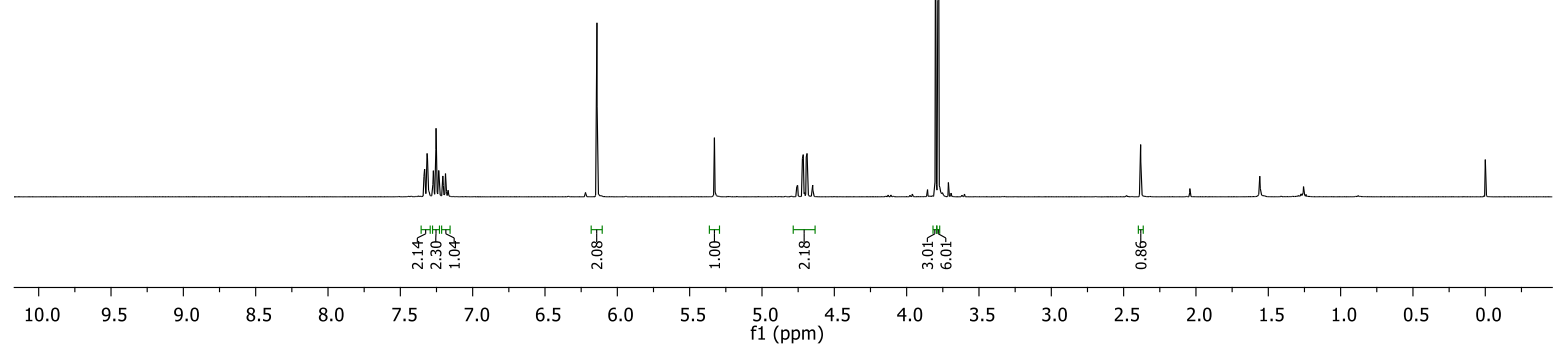




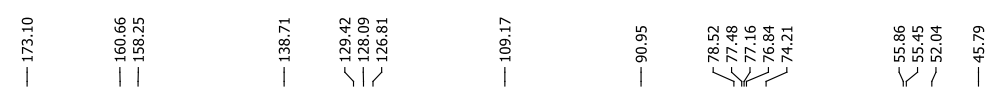

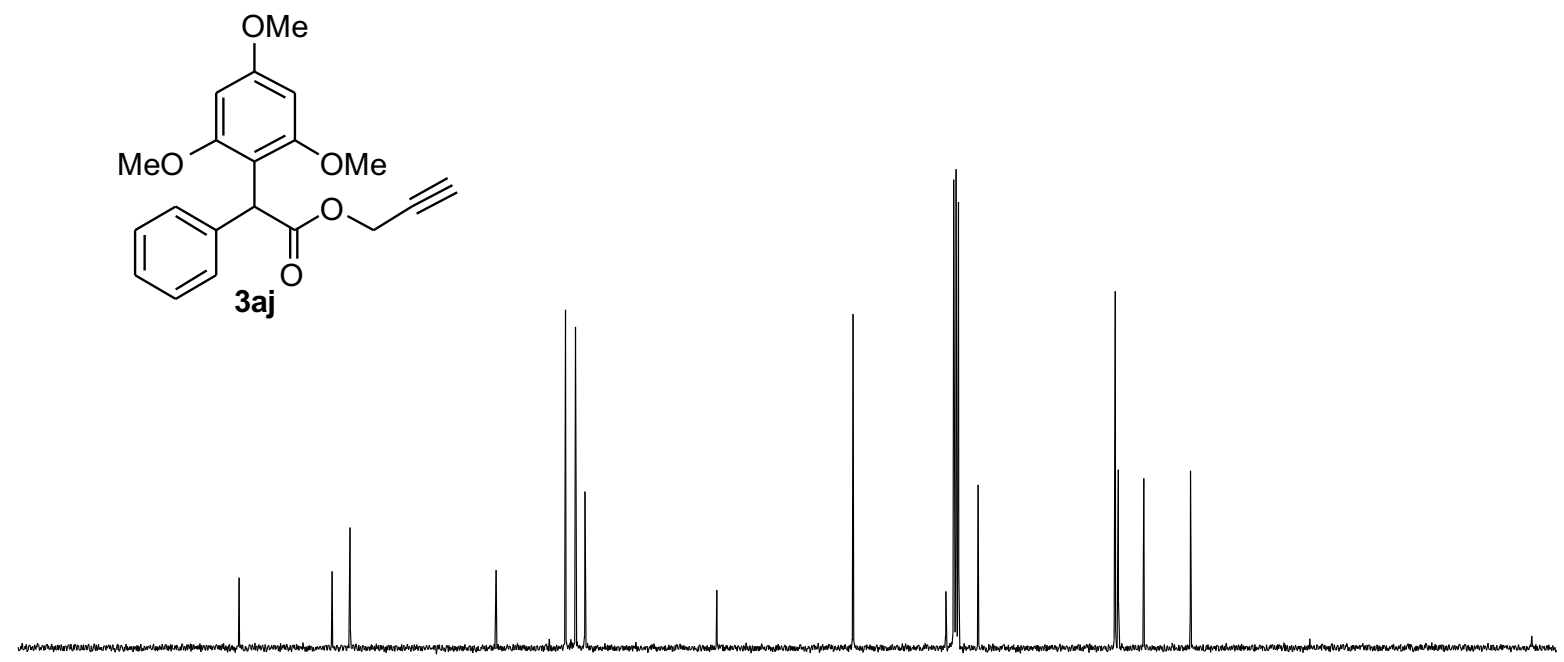

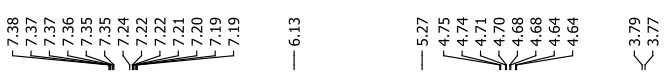

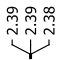<smiles>C#CCOC(=O)C(c1ccc(Br)cc1)c1c(OC)cc(OC)cc1OC</smiles>

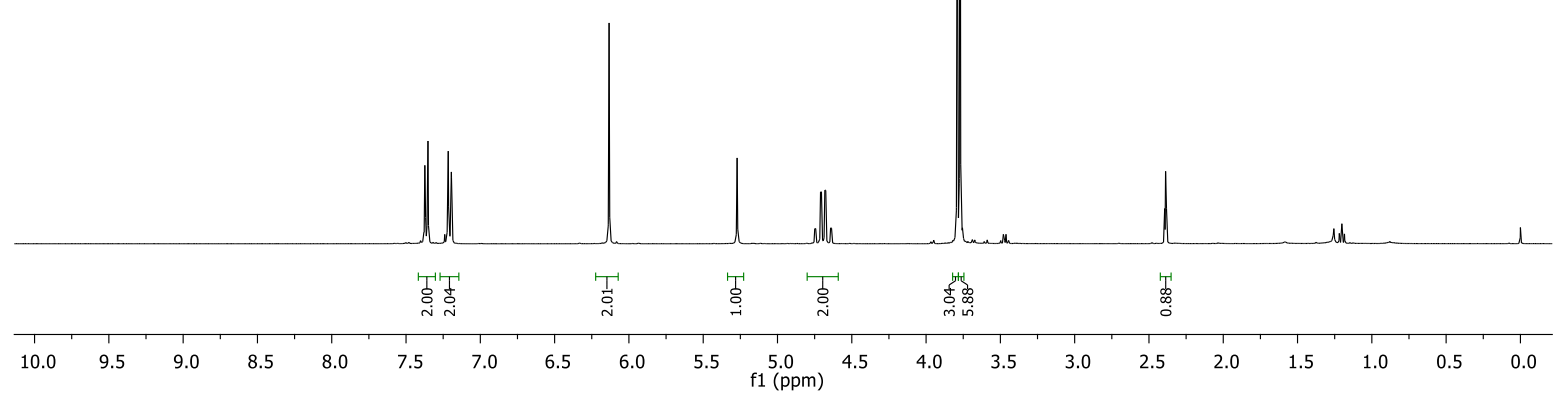




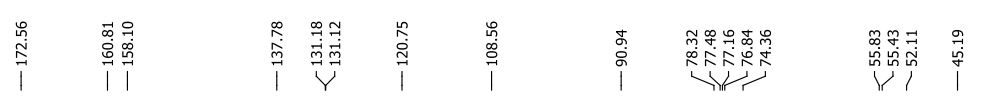<smiles>C#CCOC(=O)C(c1ccc(Br)cc1)c1c(OC)cc(OC)cc1OC</smiles>
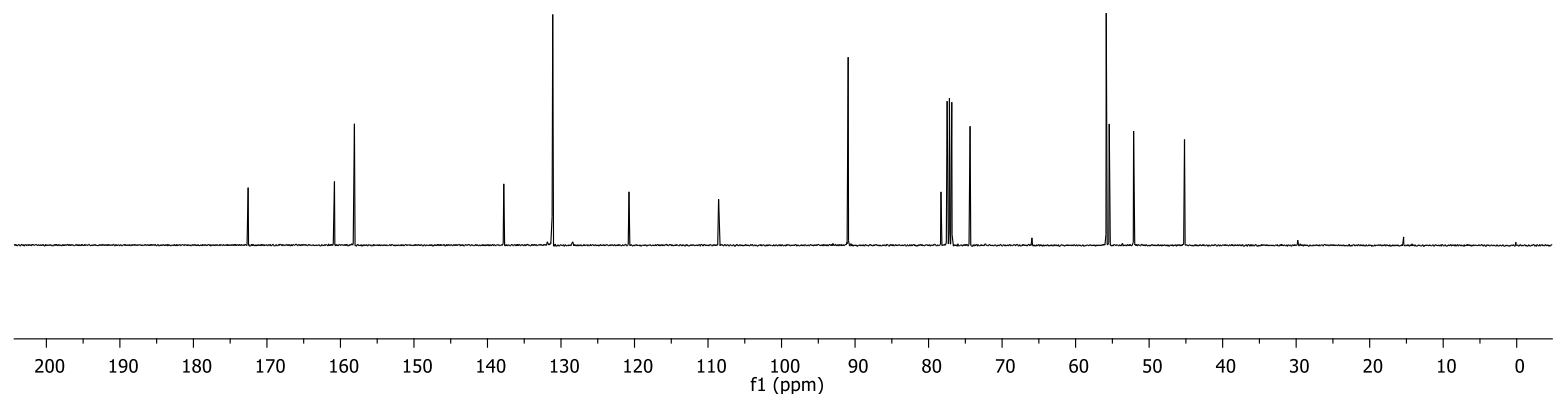

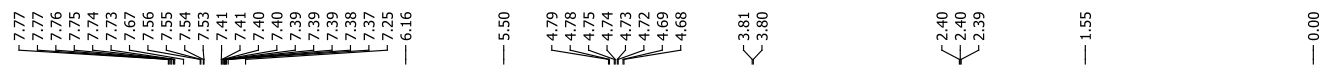<smiles>C#CCOC(=O)C(c1ccc2ccccc2c1)c1c(OC)cc(OC)cc1OC</smiles>

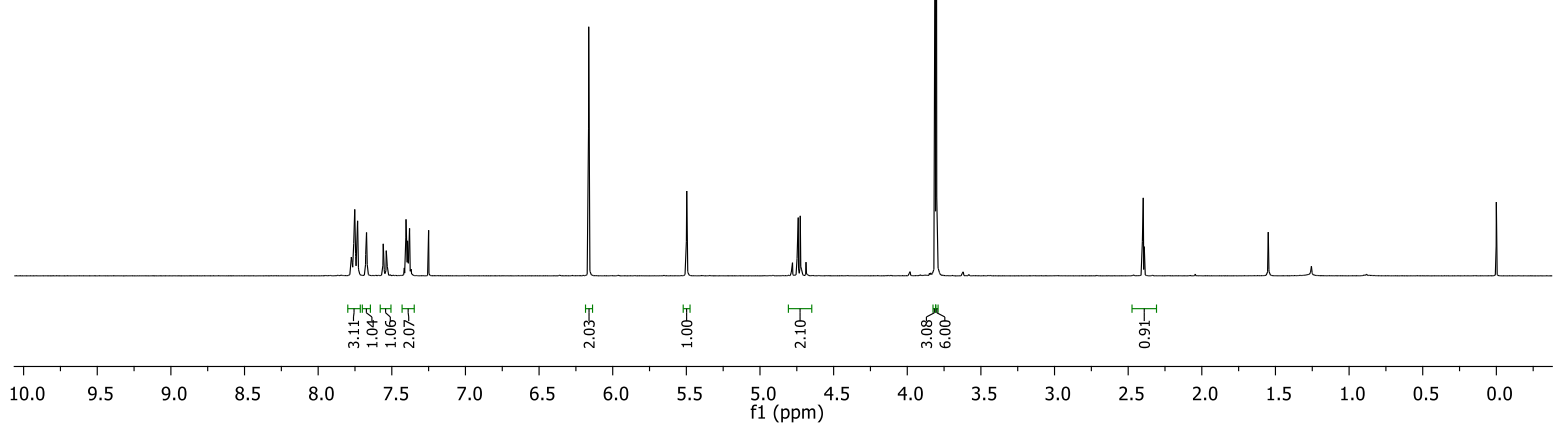




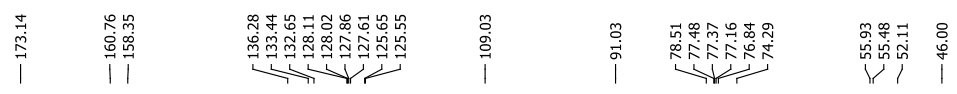
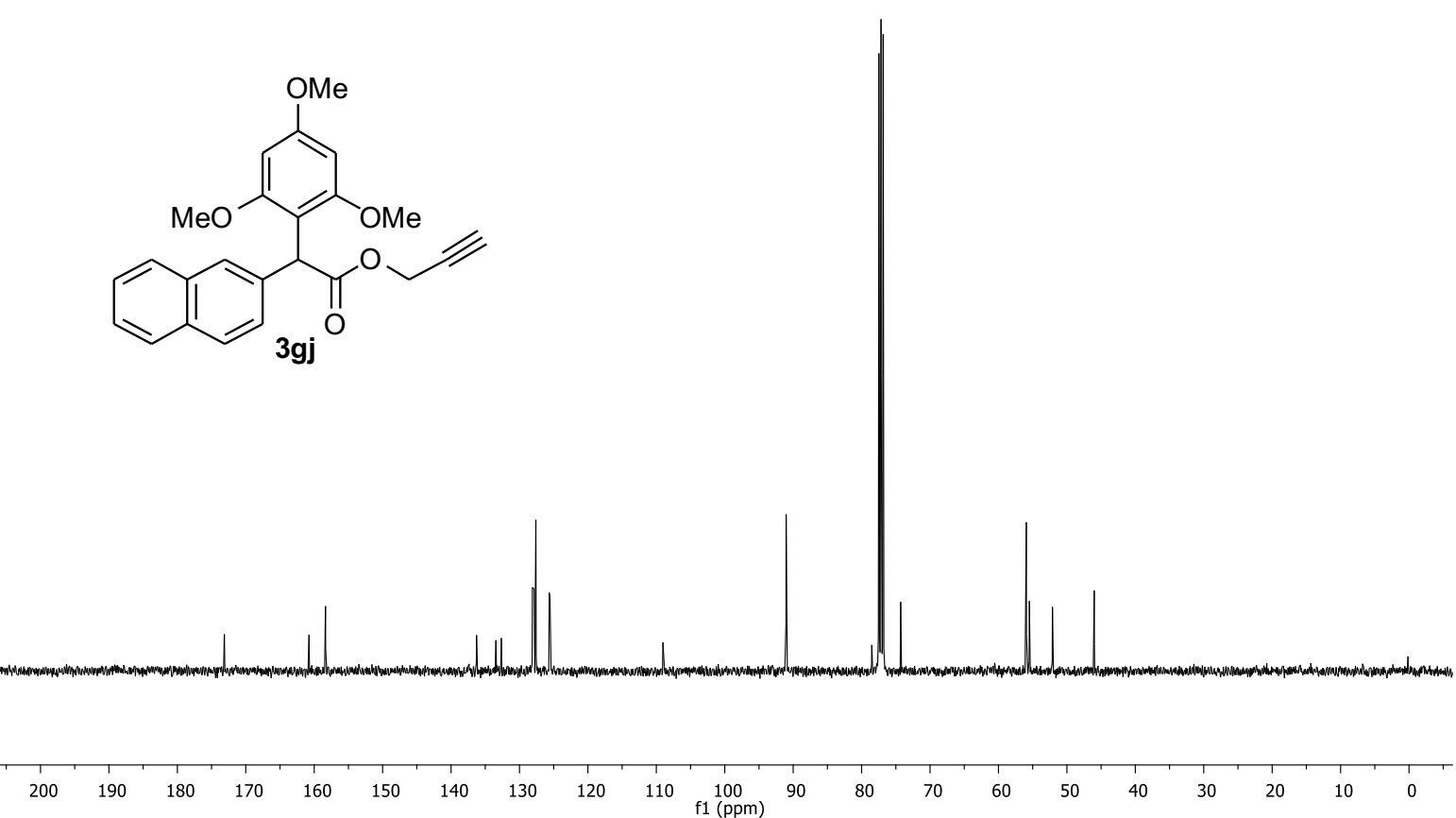

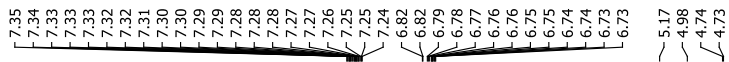

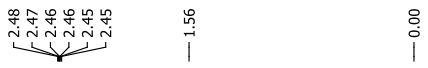<smiles>C#CCOC(=O)C(c1ccccc1)c1ccc2c(c1)OCO2</smiles>

3ak

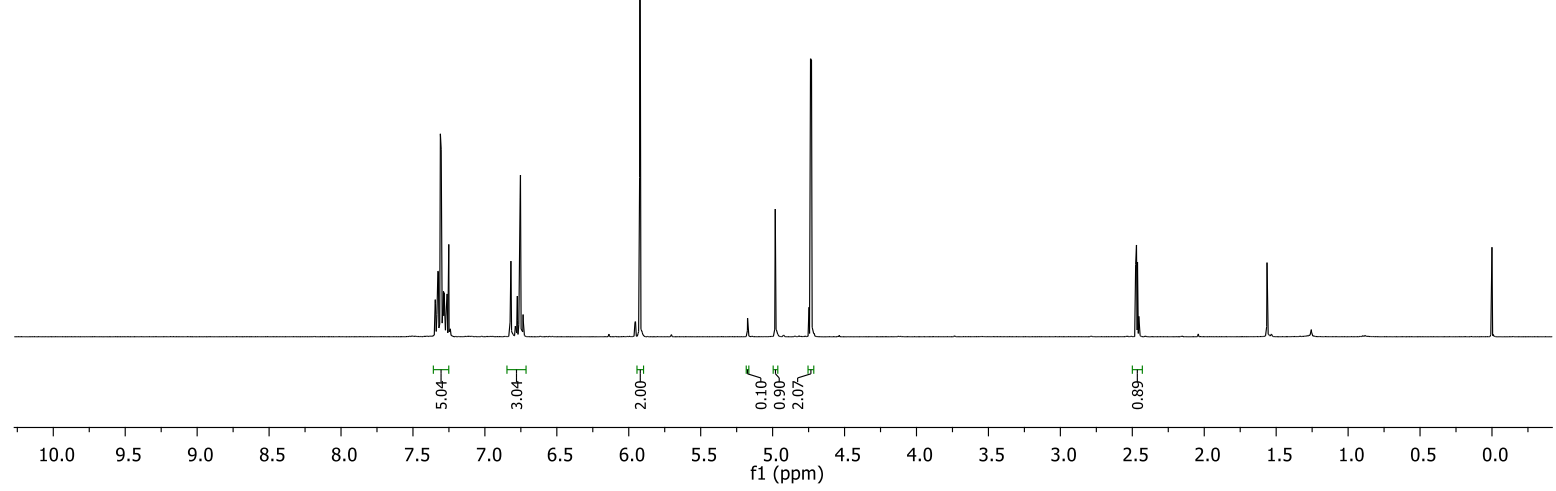




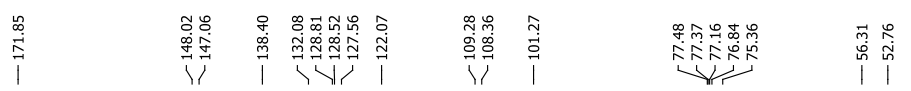

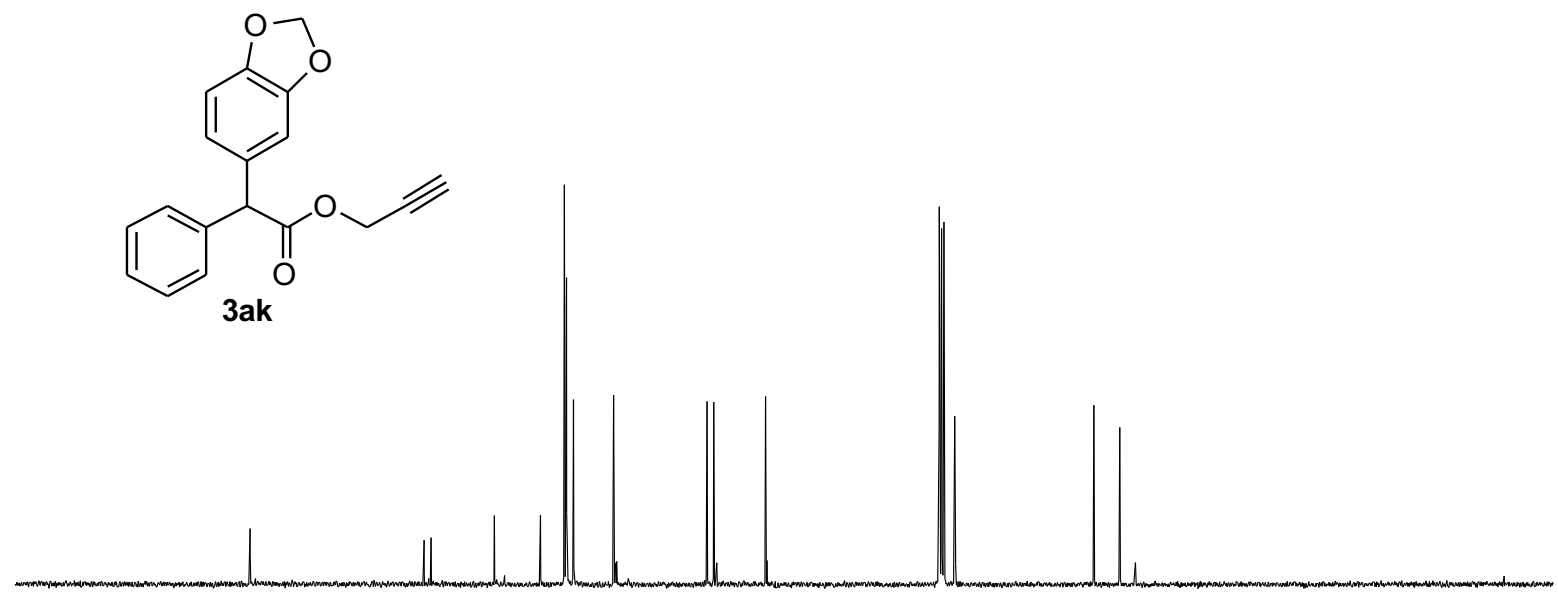

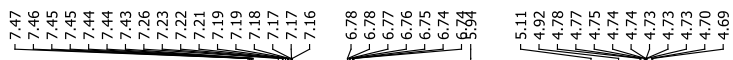

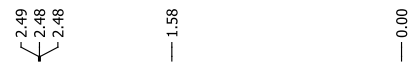

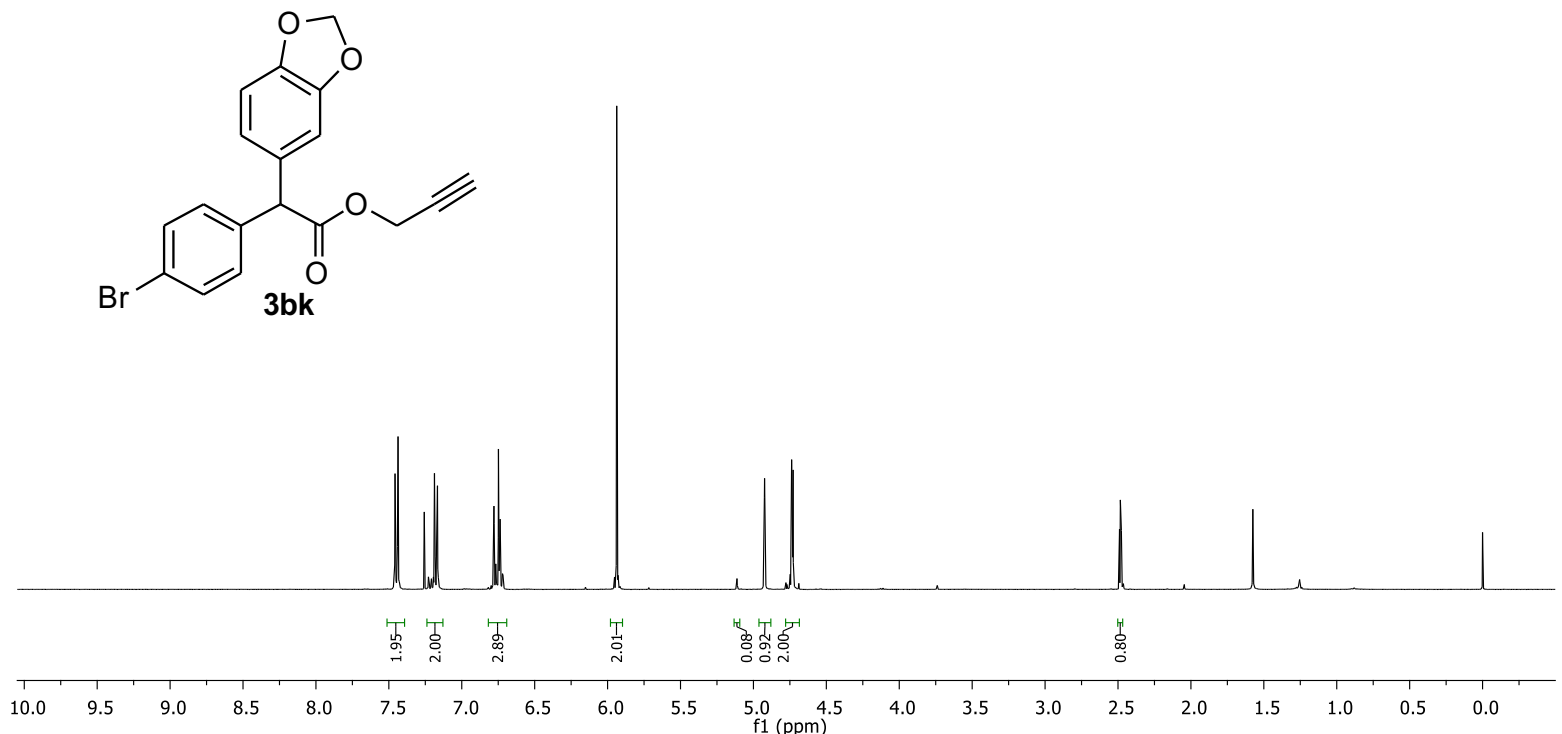




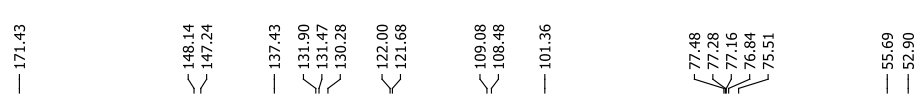<smiles>C#CCOC(=O)C(c1ccc(Br)cc1)c1ccc2c(c1)OCO2</smiles>

$\underbrace{\sqrt{n-5}}$

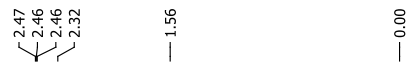

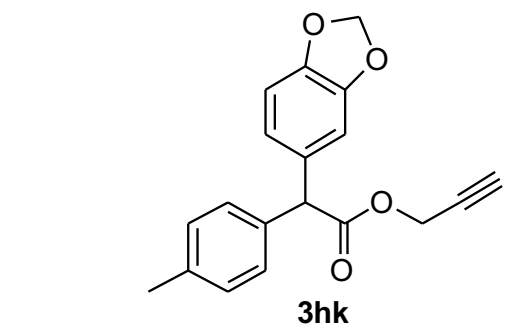

3hk

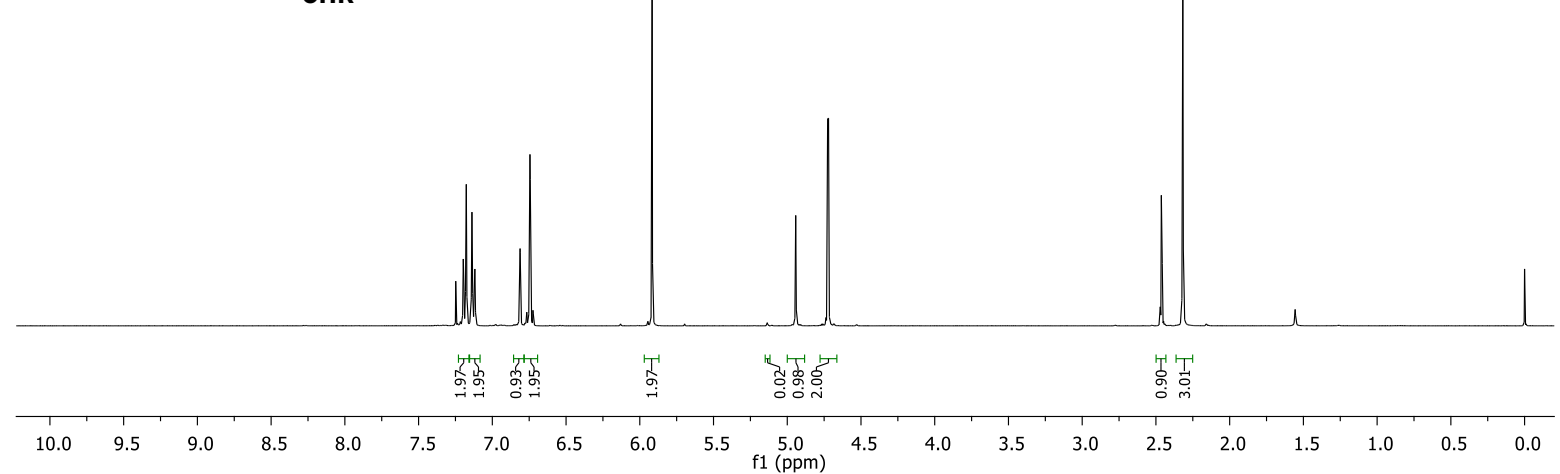



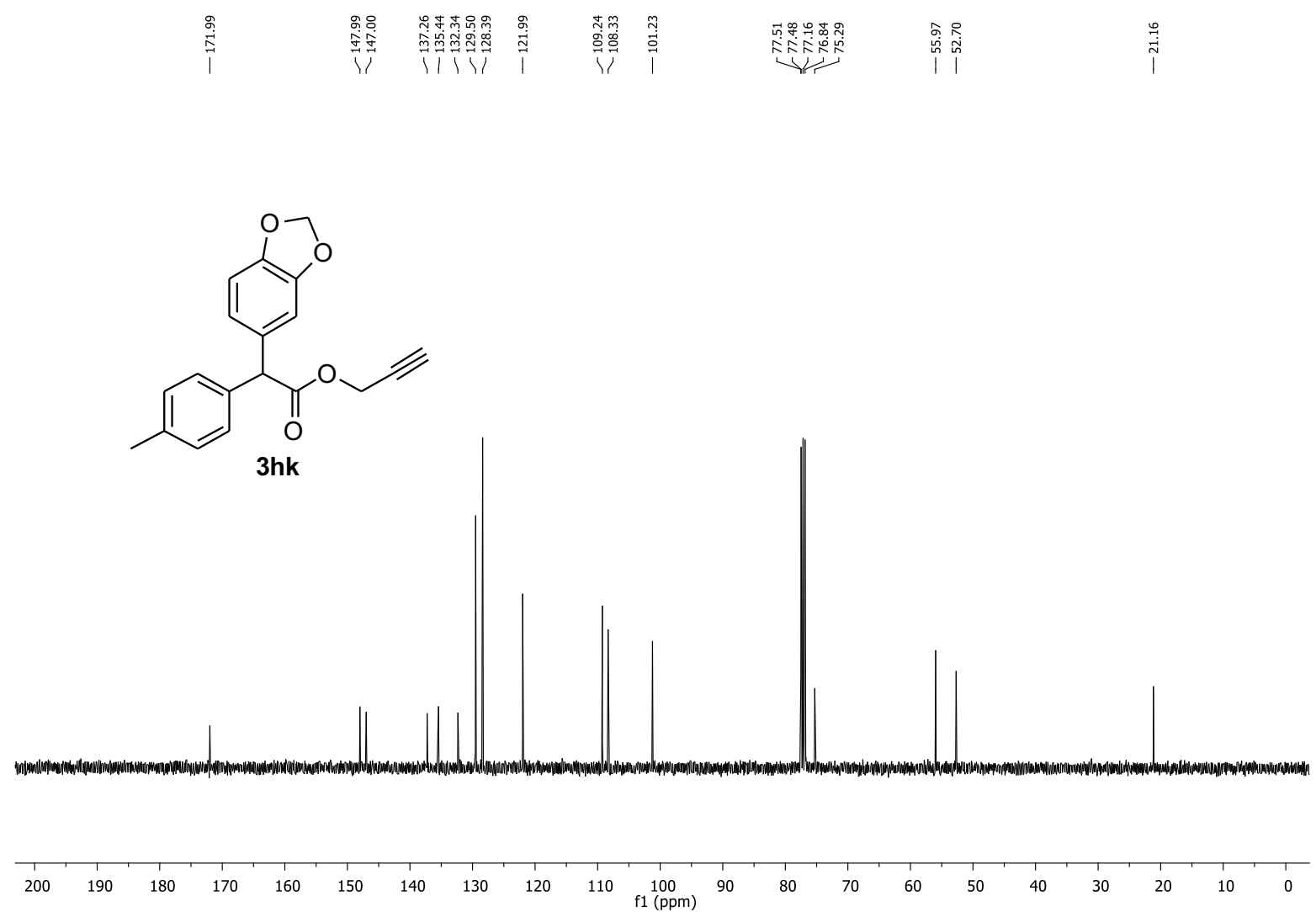

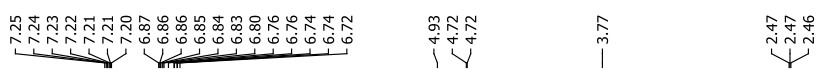

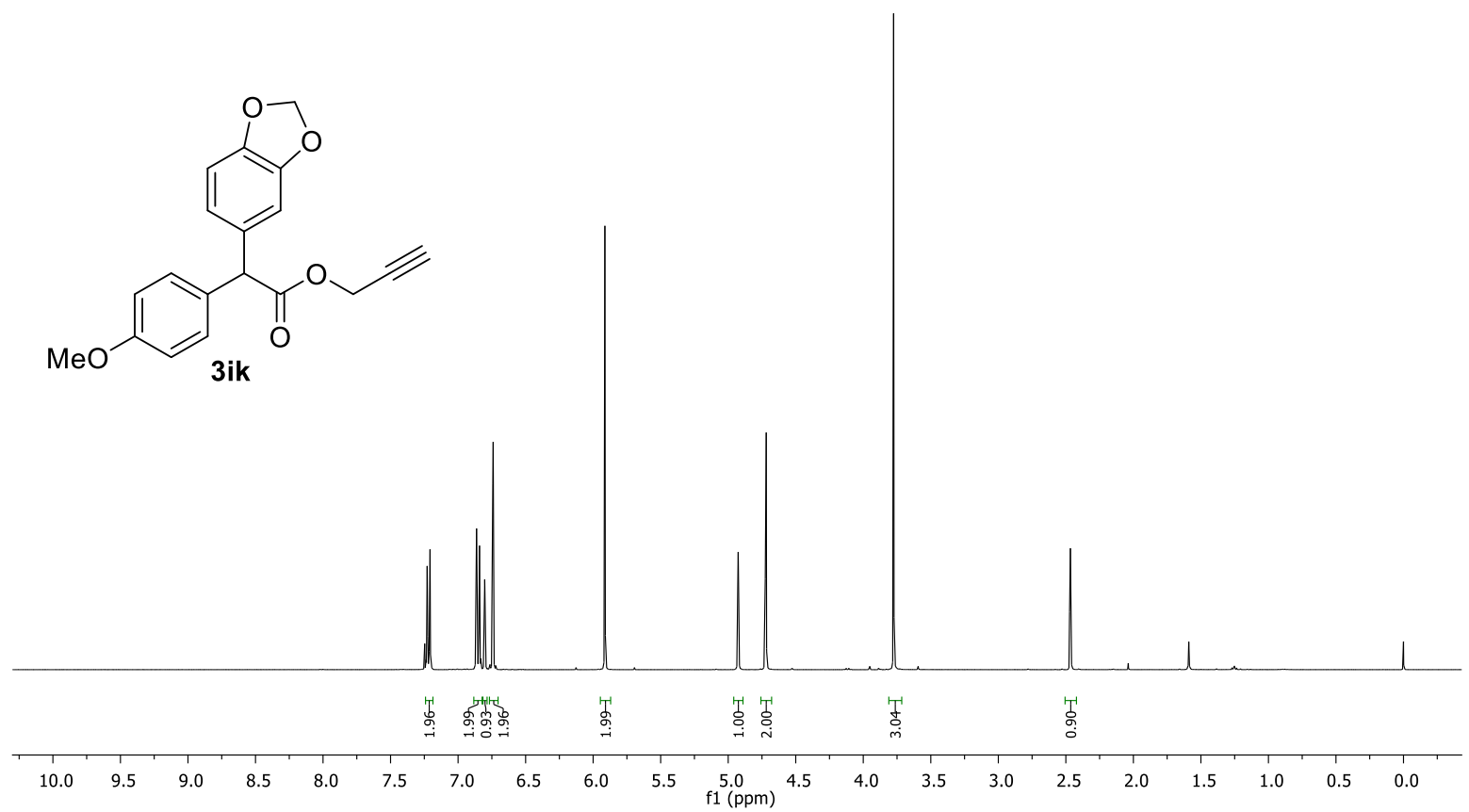


।

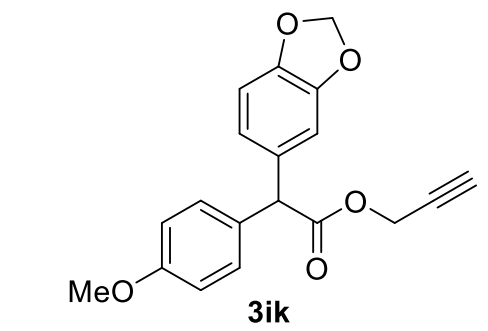

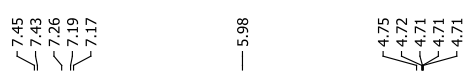

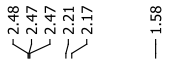<smiles>C#CCOC(=O)C(c1ccc(Br)cc1)c1cc(C)oc1C</smiles>

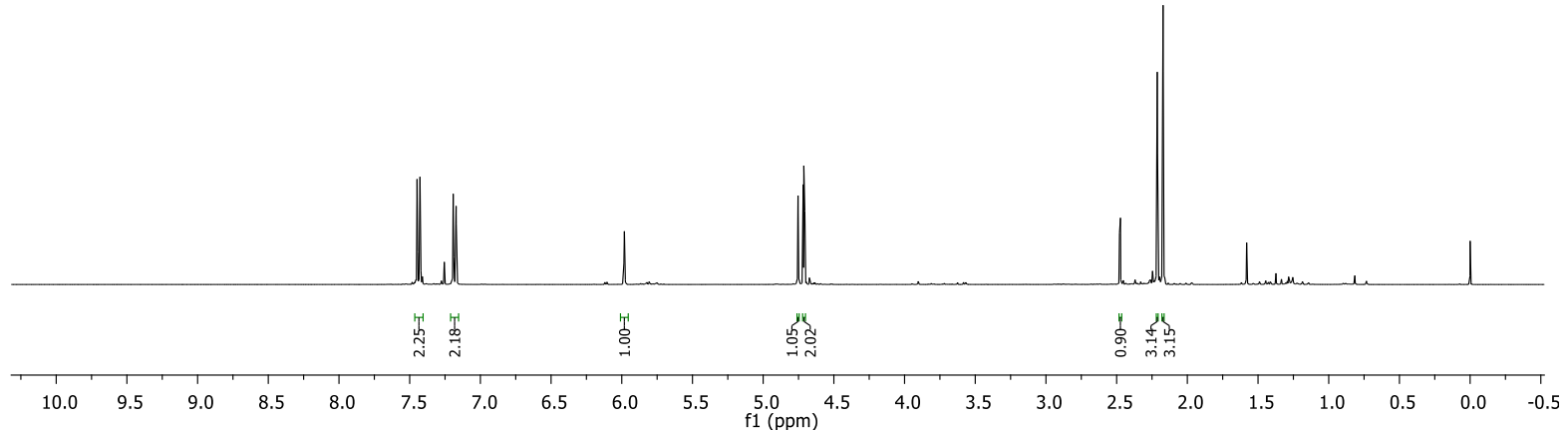



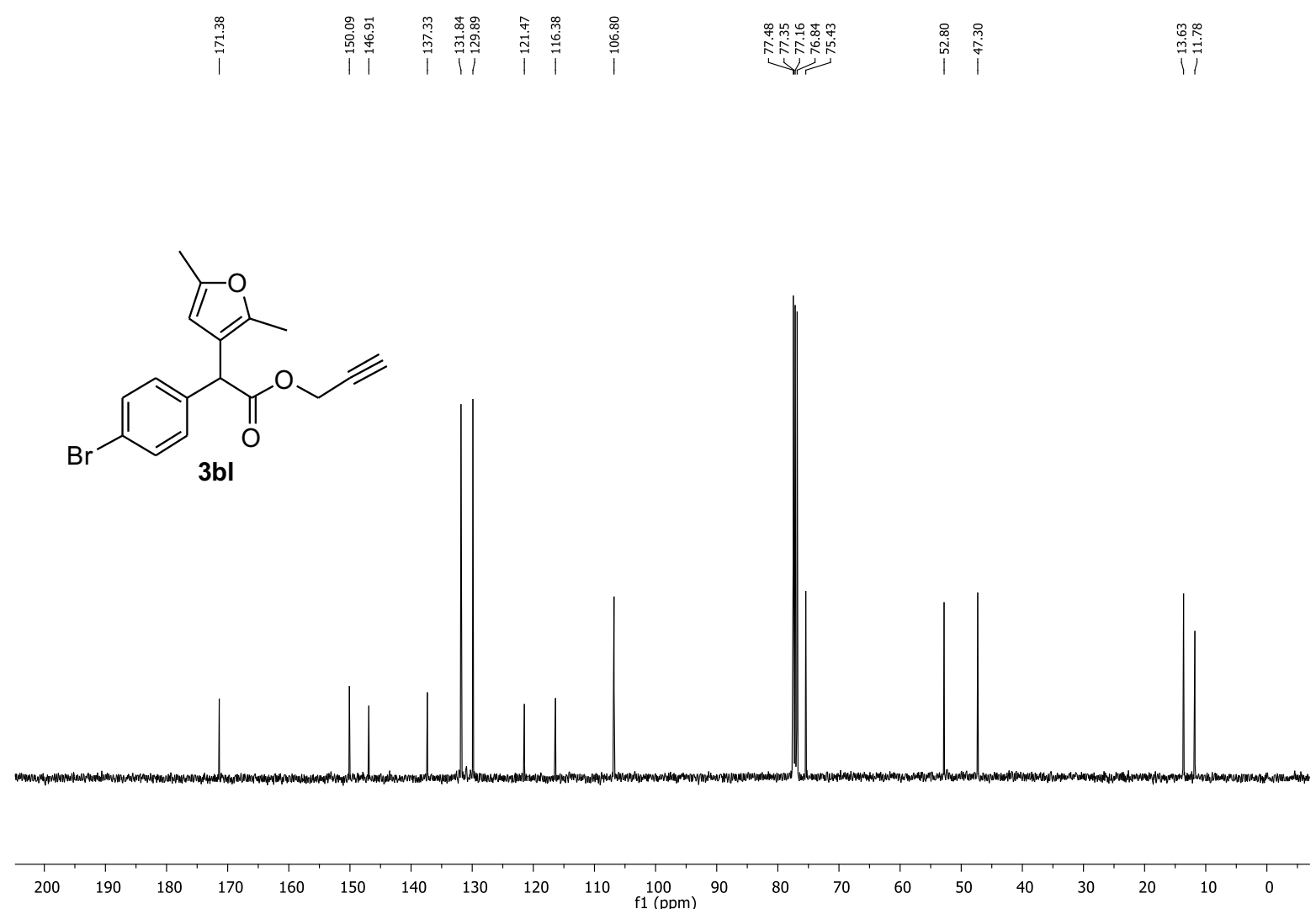

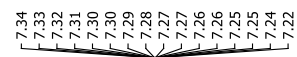

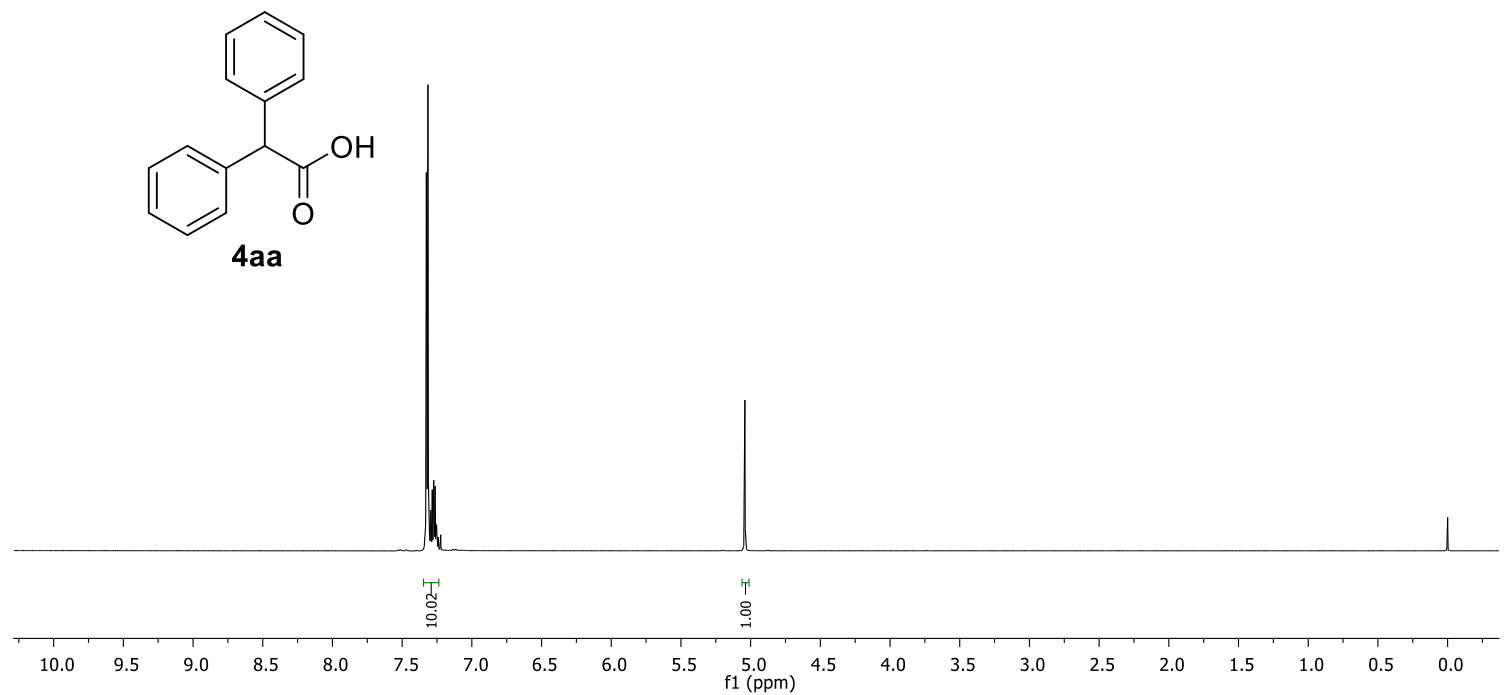




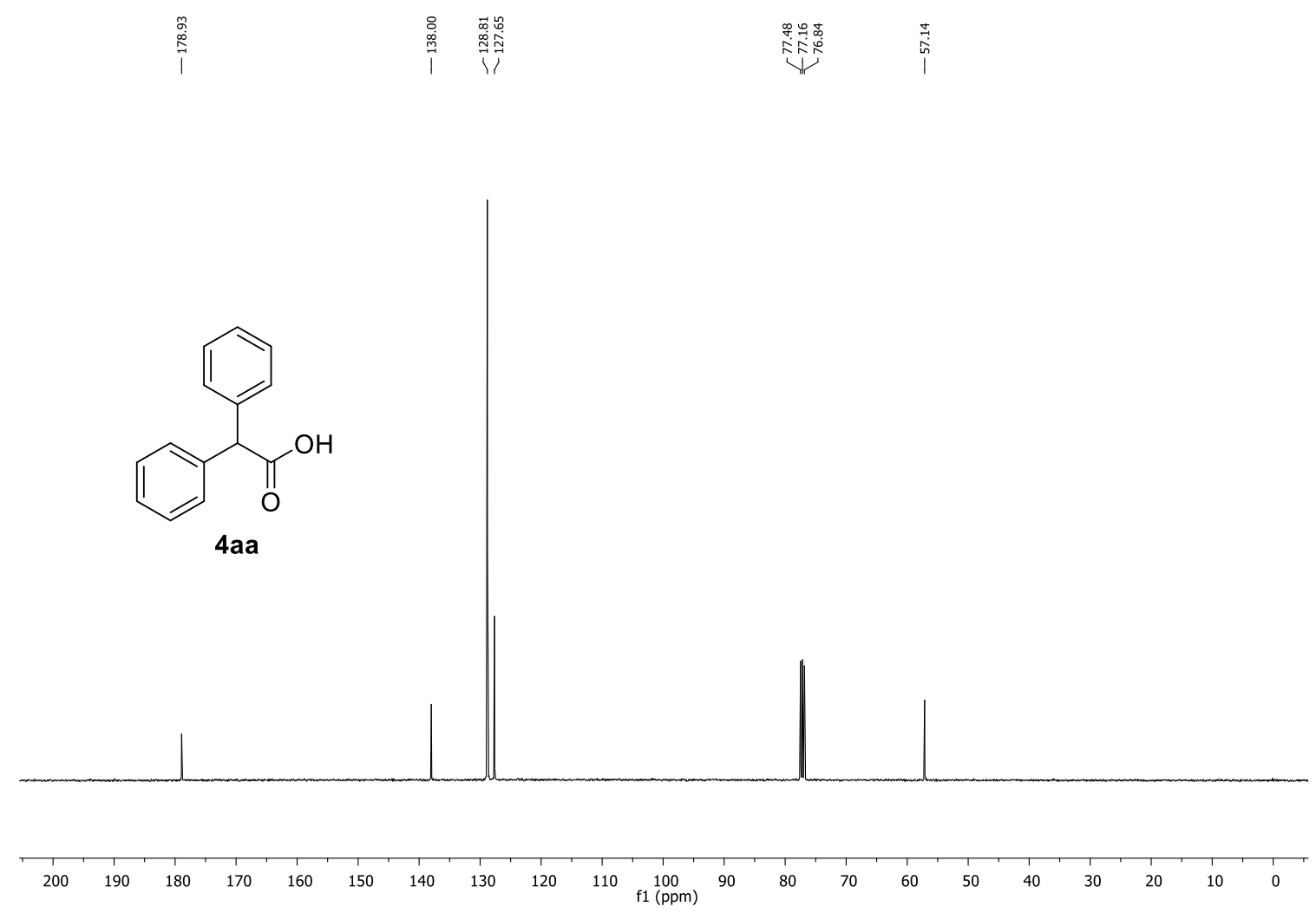




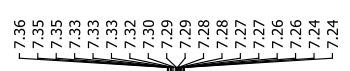

$\stackrel{\overrightarrow{0}}{i}$

i

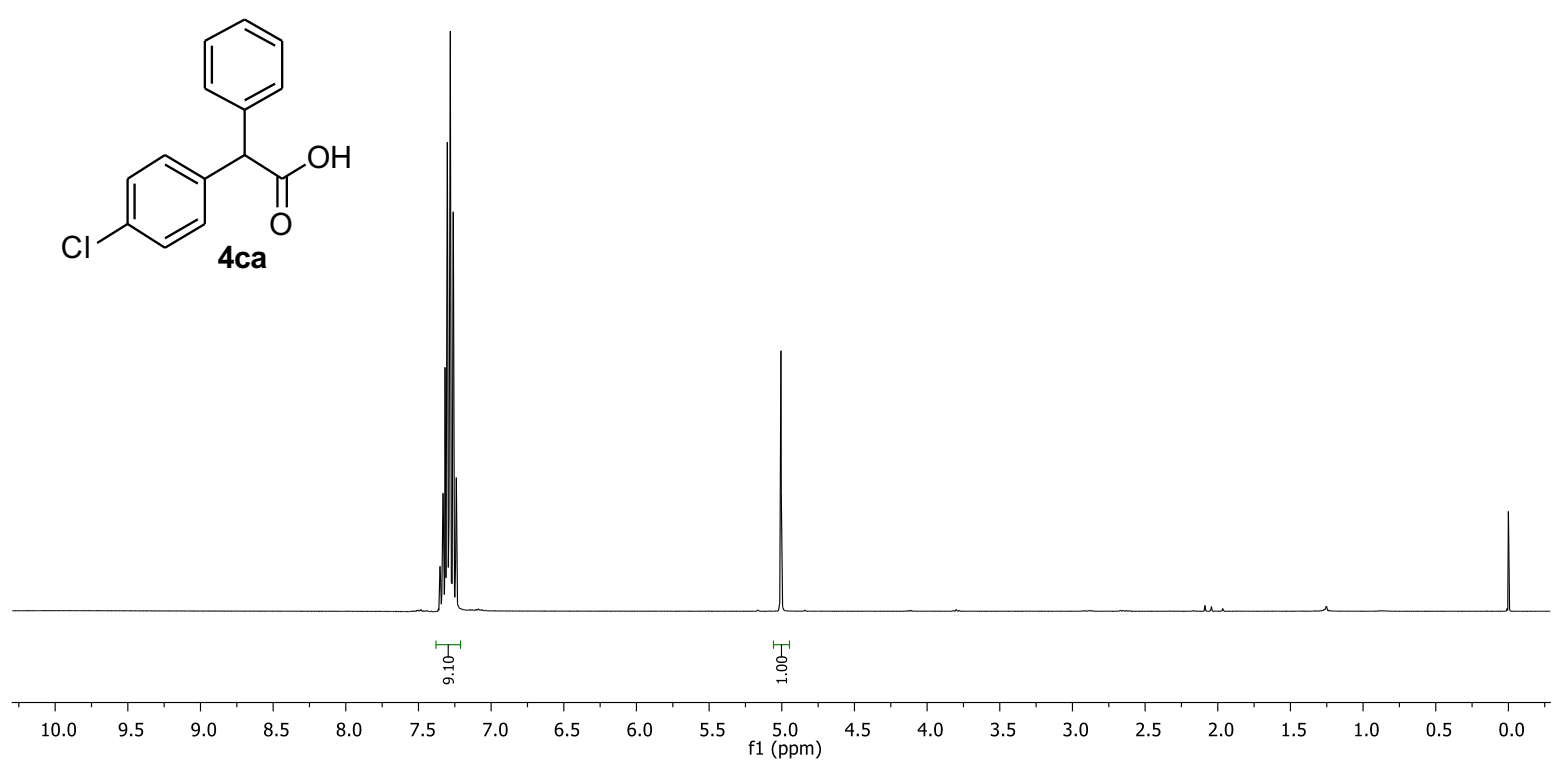

$\stackrel{\substack{0 \\ \infty}}{\stackrel{\infty}{1}}$

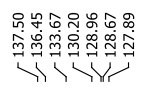

凩

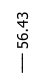
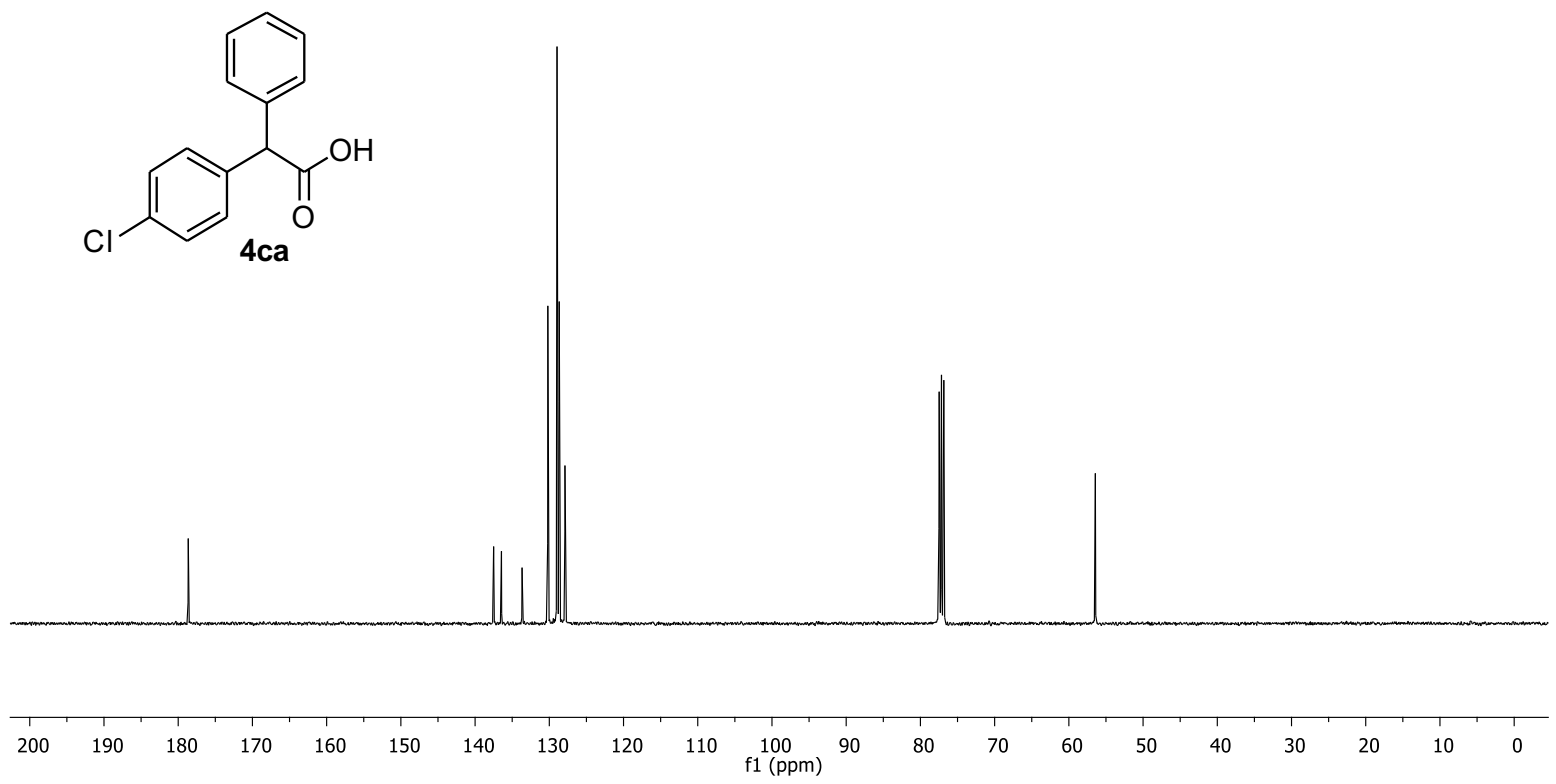

S118 
<smiles>O=C(O)C(c1ccccc1)c1ccc(Cl)c(Cl)c1</smiles>

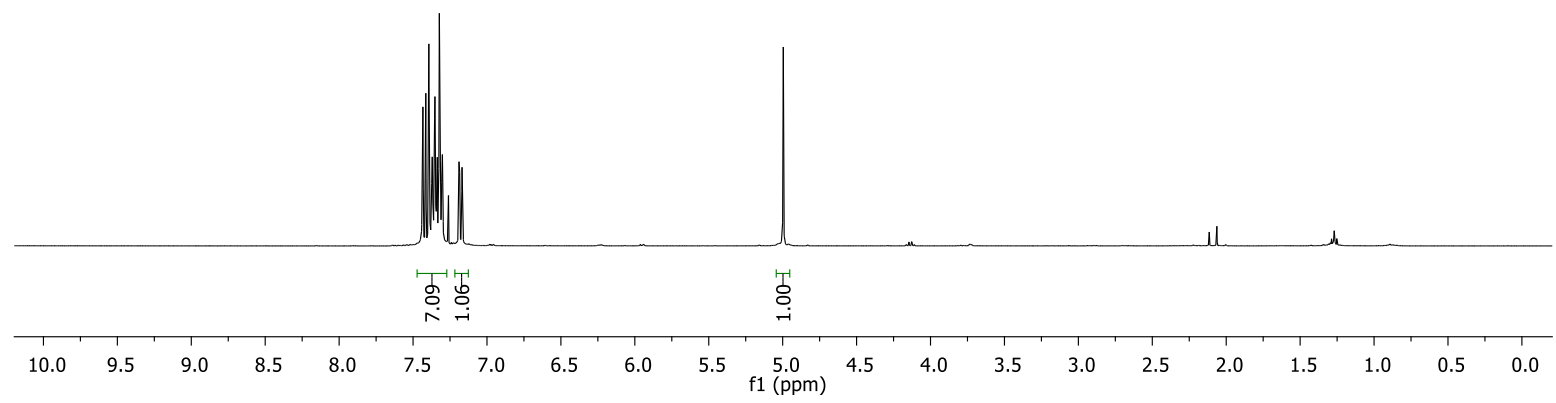

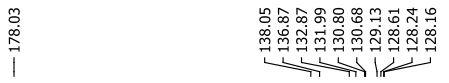

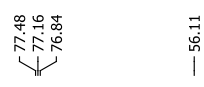<smiles>O=C(O)C(c1ccccc1)c1ccc(Cl)c(Cl)c1</smiles>

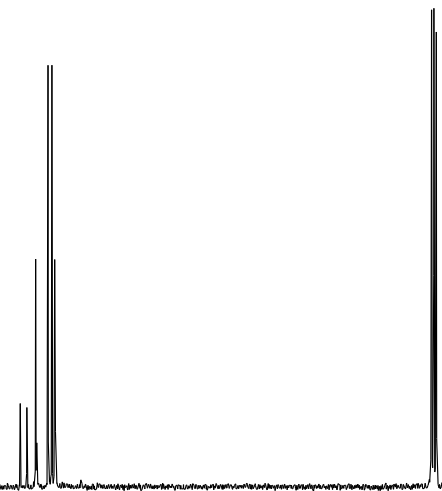

200

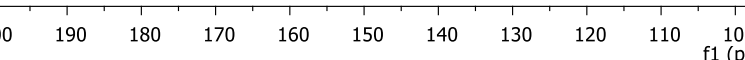
f1 1 (ppm)
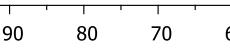

50
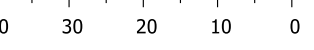


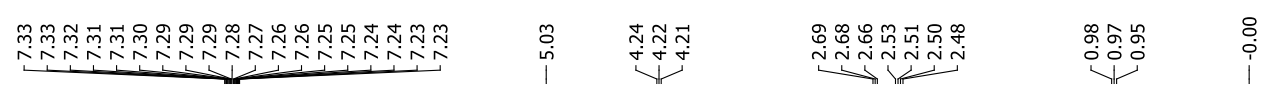

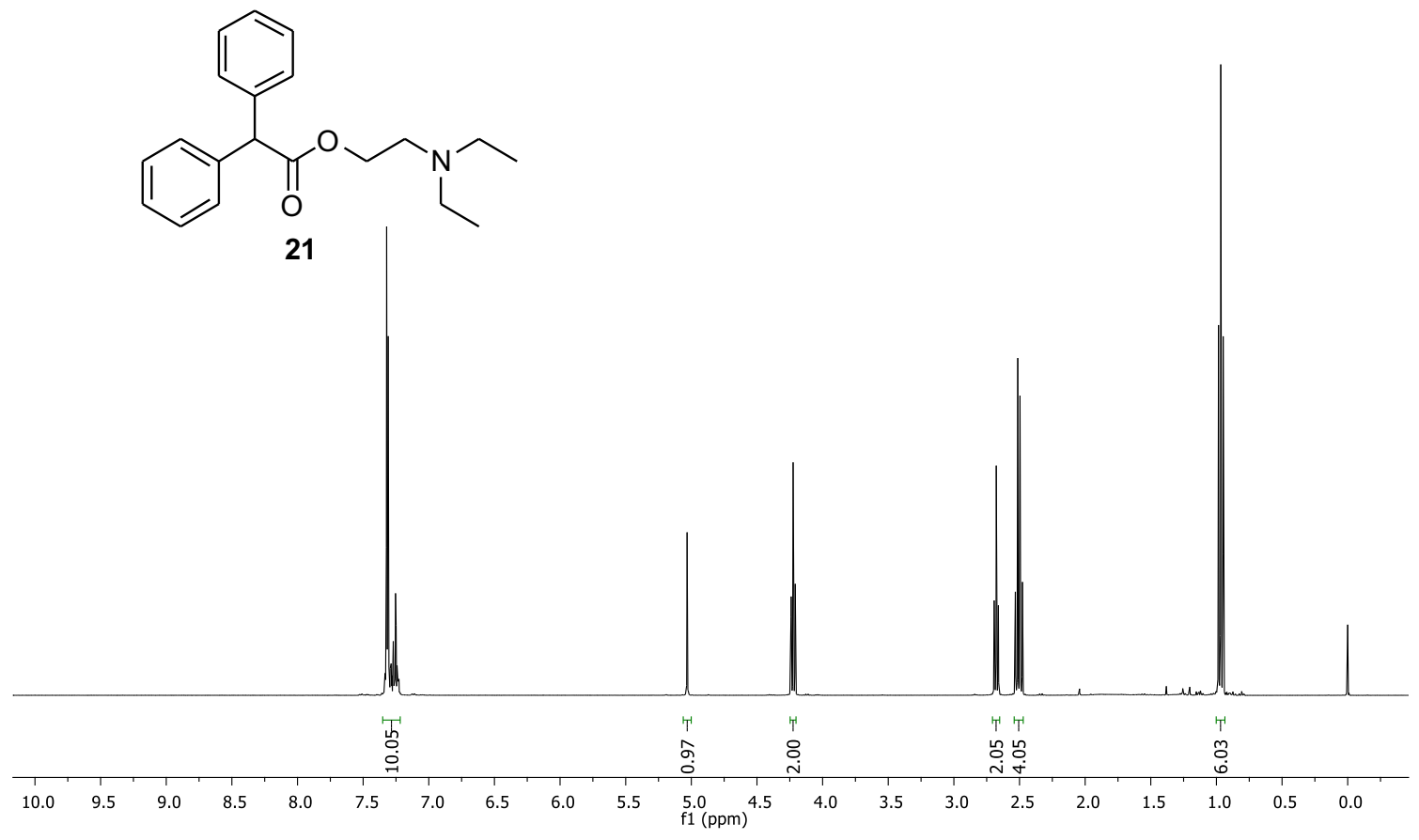

我<smiles>[Z17]C(=O)C(c1ccccc1)c1ccccc1</smiles>

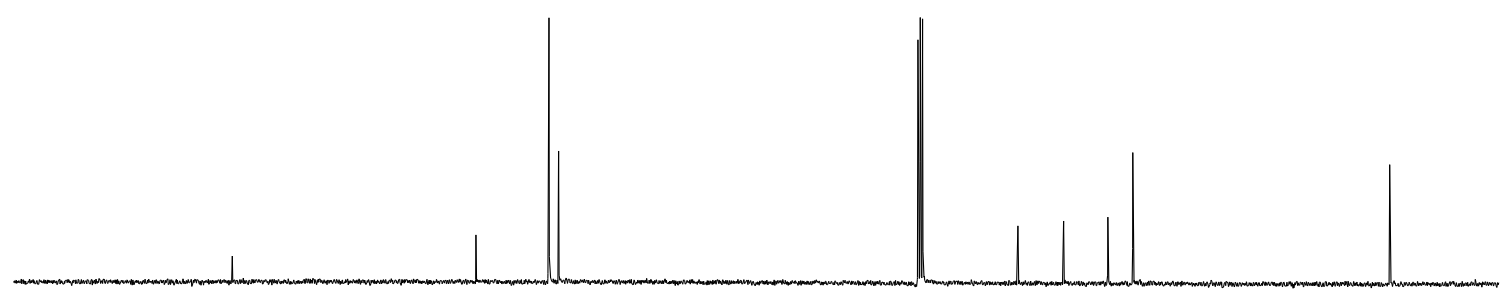

\begin{tabular}{llllllllllllllllllllll}
\hline 200 & 190 & 180 & 170 & 160 & 150 & 140 & 130 & 120 & 110 & $\begin{array}{c}100 \\
\mathrm{f} 1(\mathrm{ppm})\end{array}$ & 90 & 80 & 70 & 60 & 50 & 40 & 30 & 20 & 10 & 0
\end{tabular} 


\section{Crystal data of compound 3aa}
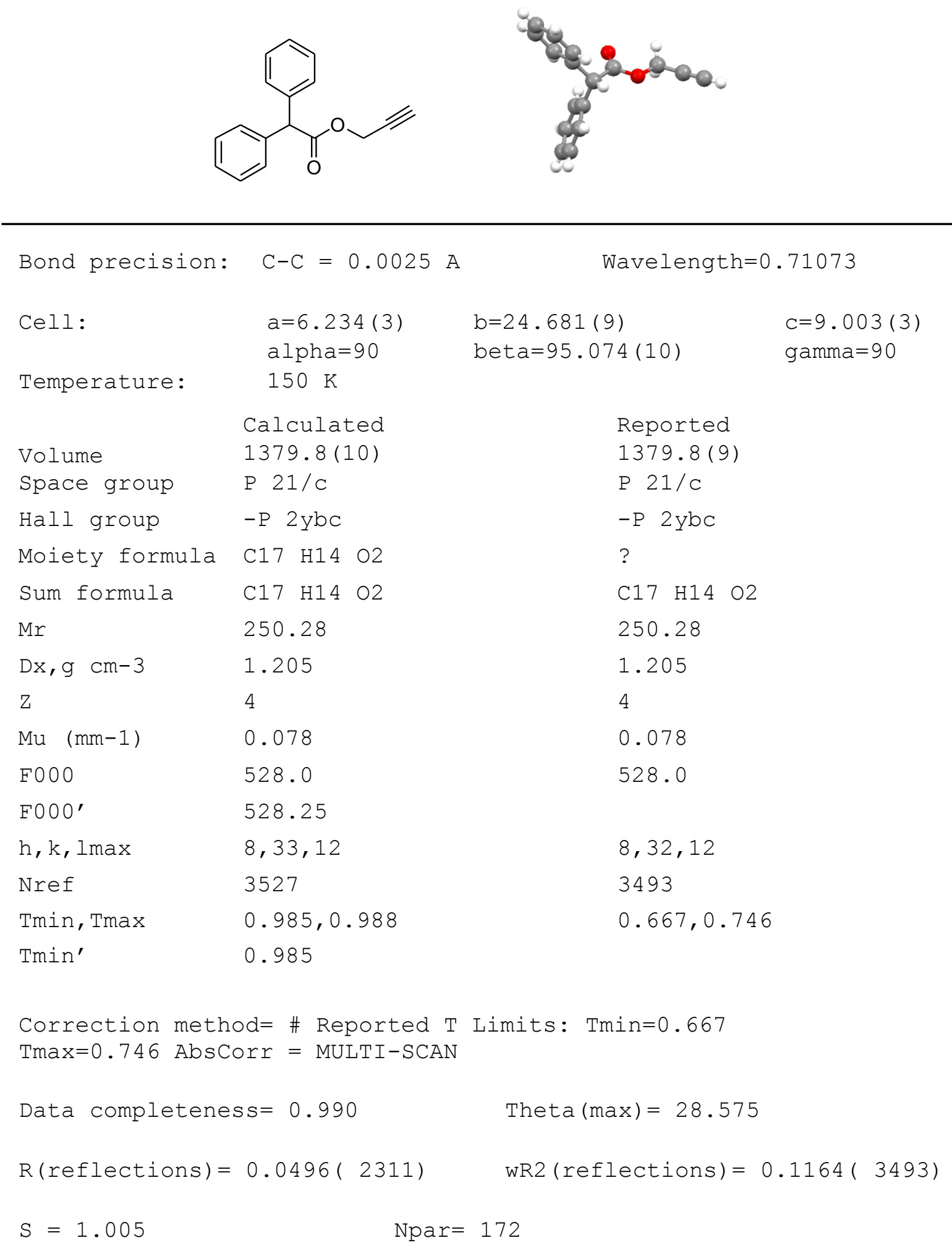


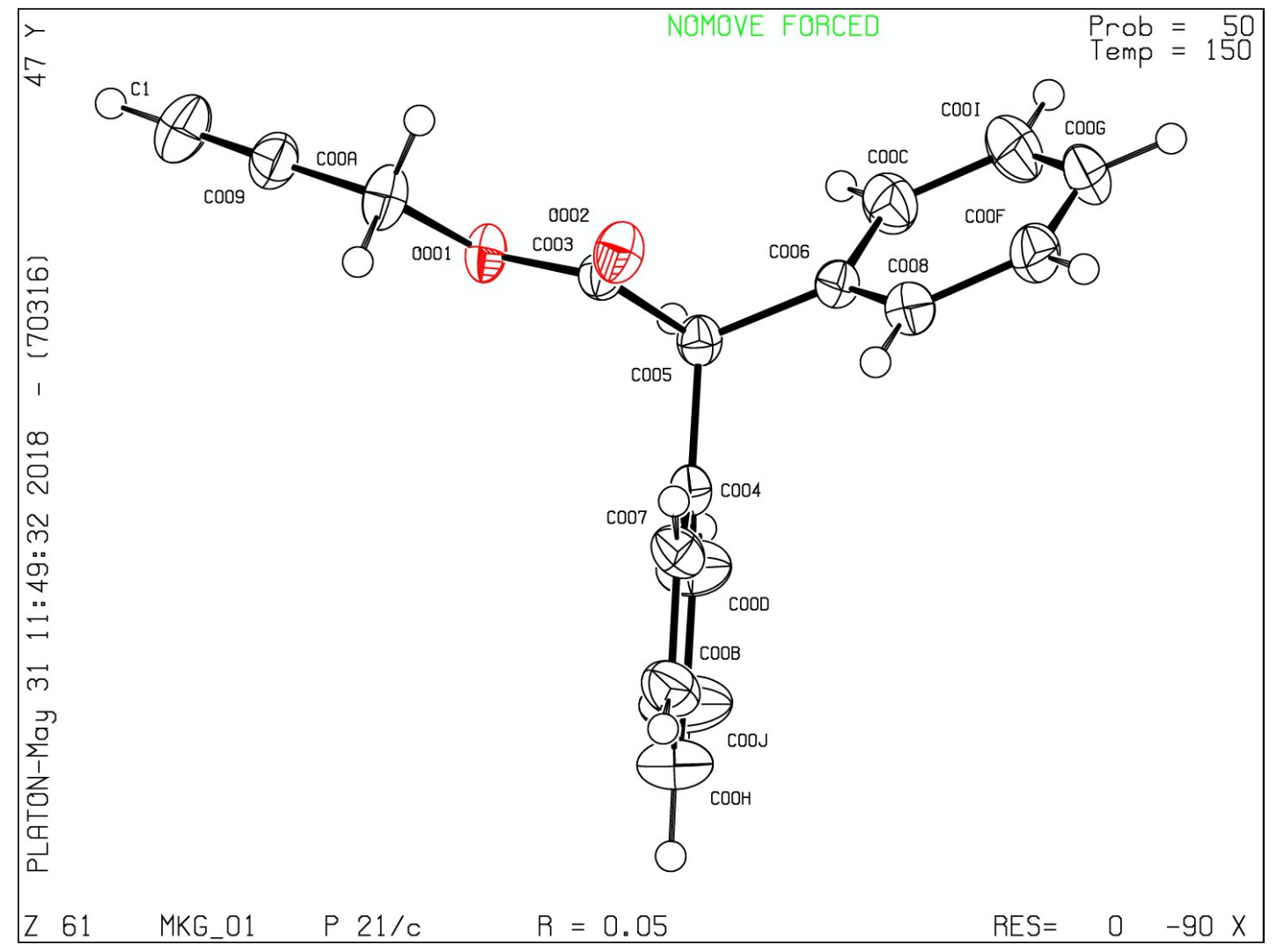


